\title{
A NUMERICAL FITTING PROCEDURE FOR THE EMBEDDED ATOM METHOD INTERATOMIC POTENTIAL AND A BRIDGED FINITE ELEMENT-MOLECULAR DYNAMICS METHOD FOR LARGE ATOMIC SYSTEMS
}

\author{
By \\ Karthik Narayan, B.Eng \\ Aerospace Engineering \\ Ryerson University, 2005
}

\begin{abstract}
A thesis presented to Ryerson University
in partial fulfillment of the requirements for the degree of

Master of Applied Science

in the Program of

Aerospace Engineering
\end{abstract}

Toronto, Ontario, Canada, 2007

(C) Karthik Narayan 2007 
UMI Number: EC53513

\section{INFORMATION TO USERS}

The quality of this reproduction is dependent upon the quality of the copy submitted. Broken or indistinct print, colored or poor quality illustrations and photographs, print bleed-through, substandard margins, and improper alignment can adversely affect reproduction.

In the unlikely event that the author did not send a complete manuscript and there are missing pages, these will be noted. Also, if unauthorized copyright material had to be removed, a note will indicate the deletion.

\section{$\mathrm{UMI}^{\circ}$}

UMI Microform EC53513

Copyright 2009 by ProQuest LLC

All rights reserved. This microform edition is protected against unauthorized copying under Title 17, United States Code.

ProQuest LLC

789 East Eisenhower Parkway

P.O. Box 1346

Ann Arbor, MI 48106-1346 
AUTHOR'S DECLARATION

I hereby declare that I am the sole author of this thesis.

I authorize Ryerson University to lend this thesis to other institutions or individuals for the purpose of scholarly research.

$$
12^{\text {TH }} \text { APR SR, } 2007
$$

I further authorize Ryerson University to reproduce this thesis by photocopying or by other means, in total or in part, at the request of other institutions or individuals for the purpose of scholarly research.

$$
\sim 12^{T H} \text { APRIL, } 2007
$$

iii 


\title{
ABSTRACT
}

\section{A Numerical Fitting Procedure for the Embedded Atom Method Interatomic Potential and a Bridged Finite Element-Molecular Dynamics Method for Large Atomic Systems}

\author{
Karthik Narayan, Master of Applied Science, Aerospace Engineering \\ Ryerson University, Toronto, 2007
}

This thesis presents a powerful numerical fitting procedure for generating Embedded Atom Method (EAM) inter-atomic potentials for pure Face Centred Cubic (FCC) and Body Centred Cubic (BCC) metals. The numerical fitting procedure involves assuming a reasonable parameterized form for a portion of the EAM potential, and then fitting the remaining portion to select thermal and elastic properties of the metal. Molecular Dynamics (MD) simulation is used to effect the fitting procedure. The procedure is used to generate an EAM potential for copper, an FCC metal. This resulting EAM potential is used to conduct MD simulations of perfect copper crystals containing voids of different geometries. Following this, a bridged Finite Element-Molecular Dynamics (FE-MD) method is presented, which can be used to simulate large atomic systems much more efficiently than MD simulation alone. The method implements a novel element discretization scheme proposed by the author that is so general that it can be applied to any system of objects interacting with each other via any potential (simple or complex, EAM or otherwise). This bridged FE-MD method is used to reanalyze the voids in the copper crystal lattice. The resulting virial stress increment patterns are found to agree remarkably with the earlier MD simulation results. Furthermore, the bridged FE-MD method is much quicker than the pure MD simulation. These two facts prove the power and usefulness of the bridged FE-MD method, and validate the proposed element discretization scheme. 


\section{ACKNOWLEDGEMENTS}

I would like to take this opportunity to first thank my thesis supervisor, Dr. Kamran Behdinan, for his support and encouragement throughout this thesis and for all his help, academic and otherwise. I would also like to thank my thesis co-supervisor, Dr. Zouheir Fawaz, for all his help and for overseeing my thesis progress. To all my friends who have stood by my side in the best and worst of times, and who have understood my incessant work on this thesis: I thank you all for your love, patience, support and understanding. To Ryerson University, where I have spent my undergraduate and graduate years fruitfully: I salute thee. To all other people who have helped me within and without university: I thank you all, and wish you all the very best. Lastly, but most importantly, to my parents, without whose unconditional love, patience, support, understanding, guidance, and belief in my abilities, I would have never come this far in life: I thank you both, and I dedicate this thesis to you. 


\section{TABLE OF CONTENTS}

1. INTRODUCTION.......................................................................................................... 1

2. MOLECULAR DYNAMICS .................................................................................... 5

2.1 Introduction to Molecular Dynamics ............................................................... 5

2.2 The Embedded Atom Method Inter-Atomic Potential for Metals ......................... 6

2.3 The General Molecular Dynamics Algorithm ......................................................... 8

2.4 Custom Molecular Dynamics Code MD_V_7_00............................................... 10

2.4.1 Program Execution Flowcharts.................................................................... 11

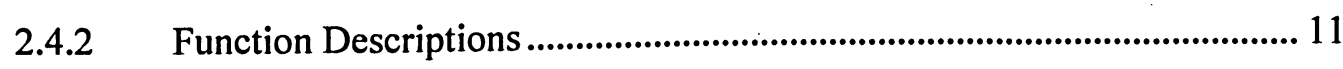

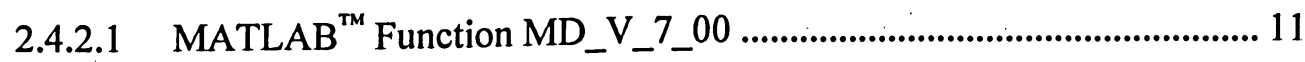

2.4.2.2 MATLAB ${ }^{\text {TM }}$ Functions FCC_Gen_V_7_00 and BCC_Gen_V_7_00... 14

2.4.2.3 MATLAB ${ }^{\mathrm{TM}}$ Functions CRACK_VOID_V_7_00..................................... 15

2.4.2.4 MATLAB ${ }^{\mathrm{TM}}$ Function EAM_Range_V_7_00......................................... 16

2.4.2.5 MATLAB ${ }^{\mathrm{TM}}$ Function EAM_Poly_Fit_V_7_00 .................................... 16

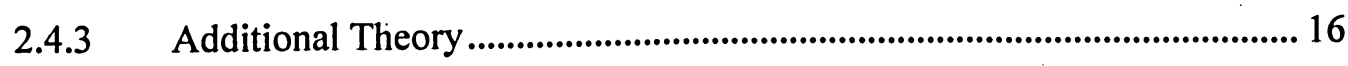

2.4.3.1 Average Stress Calculation: The Virial Stress........................................ 16

2.4.3.2 Hydrostatic Pressure and Equilibrium Lattice Constant....................... 18

2.4.3.3 Engineering Strain Tensor for a Desired Principal Virial Stress Tensor

2.4.3.4 Periodic Boundary Condition in Molecular Dynamics........................... 21

2.4.3.5 Temperature Clamping in Molecular Dynamics.................................... 22

2.5 Testing of Custom Molecular Dynamics Code: Momentum and Energy Conservation for Single Unit Cell (No Periodic Boundary Condition)....... 23

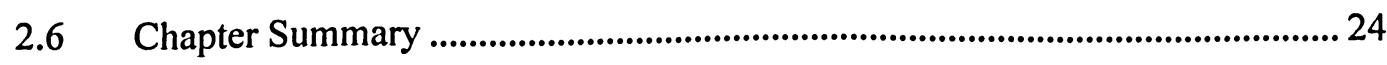

3. EMBEDDED ATOM METHOD POTENTIAL FITTING PROCEDURE FOR PURE FCC AND BCC METALS.................................................................... 27 
3.1 Introduction to Proposed Embedded Atom Method Potential Fitting Procedure 27

3.2 Molecular Dynamics (MD) Code for Numerical EAM Potential Fitting ......... 28

3.3 Essential Components of the Proposed EAM Potential Fitting Procedure....... 28

3.3.1 Relationship between Temperature and Atomic Velocity ........................ 28

3.3.2 Pair Potential for the EAM Potential: The Lennard-Jones Potential ........ 29

3.3.3 Atomic Electron Density Calculation using WinGAMESS ...................... 30

3.3.4 Varying the Total Electron Density during the EAM Fitting Process ...... 31

3.3.5 Fitting the Cohesive Energy of the Metal ................................................ 33

3.4 Proposed Numerical EAM Potential Fitting Procedure...................................... 33

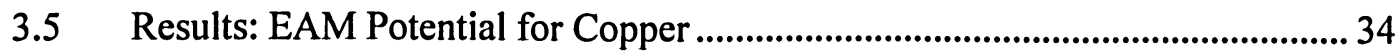

3.6 EAM Potential Testing: Thermal and Elastic Properties of Single Crystal

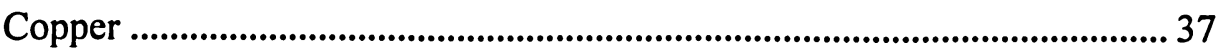

3.6.1 Test Case 1: Lattice Constants of Copper Single Crystal at Various

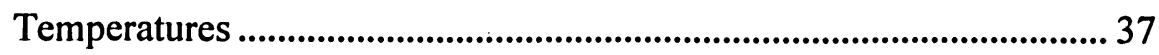

3.6.2 Test Case 2: Uniaxial Properties of Copper Single Crystal at 293K ........ 38

3.6.3 Test Case 3: Hydrostatic Elasticity of Single Crystal Copper at 293K .... 40

3.6.4 Test Case 4: Shear Modulus of Copper at 293K.......................................... 42

3.6.5 Test Case 5: Isothermal Young's Modulus and Poisson's Ratio of Copper

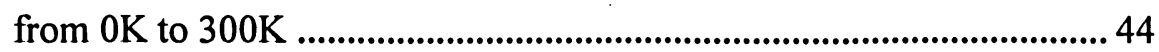

3.6.6 Summary of Results for Anisotropic Mechanical Properties of Single

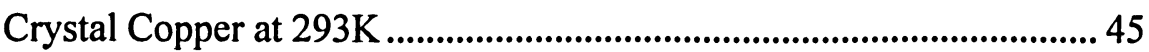

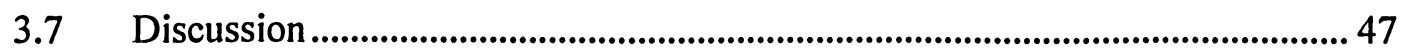

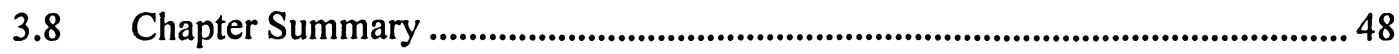

4. APPLICATION OF MOLECULAR DYNAMICS WITH THE EMBEDDED ATOM METHOD POTENTIAL............................................................. 49

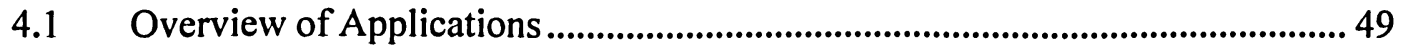

4.2 Application 1: Elastically Stressed Infinite, Perfectly Cylindrical Void in a Perfect Copper Crystal Lattice .................................................................... 50

4.2.1 Application Objective ................................................................................. 50 
4.2.2 Infinite Copper Lattice Specifications ...................................................... 50

4.2.3 Core MD Simulation Parameters ................................................................ 52

4.2.4 Application 1 Results: Virial Stress Increment Plots................................... 54

4.3 Application 2: Elastically Stressed Infinite, Semi-Cylindrical Notch at the Free Surface of a Perfect Copper Crystal Lattice ..................................................5

4.3.1 Application Objective ............................................................................... 57

4.3.2 Semi-Infinite Copper Lattice Specifications................................................. 58

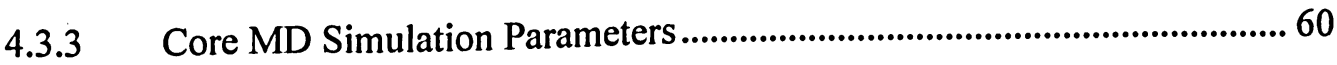

4.3.4 Application 2 Results: Stress Increment Plots............................................. 61

4.4 Application 3: Elastically Stressed Infinite, Elliptical Void in a Perfect Copper

Crystal Lattice.............................................................................................. 65

4.4.1 Application Objective ................................................................................ 65

4.4.2 Infinite Copper Lattice Specifications .......................................................... 65

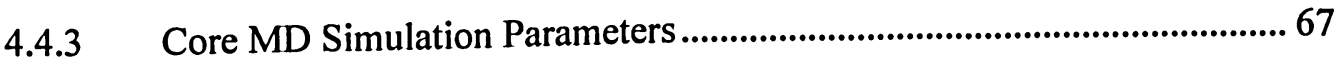

4.4.4 Application 3 Results: Stress Increment Plots.............................................. 69

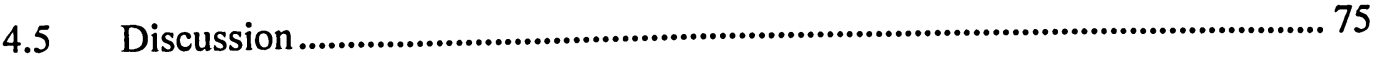

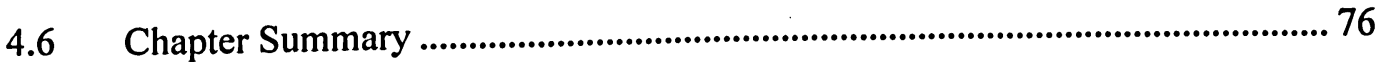

\section{A BRIDGED FINITE ELEMENT-MOLECULAR DYNAMICS METHOD} FOR LARGE ATOMIC SYSTEMS................................................. 77

5.1 Introduction to Bridged Method ......................................................................... 77

5.2 General Form of Finite Element Stiffness Equation........................................... 78

5.3 FE Boundary Conditions vs. MD Periodic Boundary Conditions...................... 81

5.4 Proposed Element Discretization Scheme for Bridged FE-MD Method........... 84

5.4.1 Conventional FEM Element Discretization Scheme .................................... 84

5.4.2 Proposed Element Discretization Scheme .................................................... 87

5.4.3 Comparison of Discretization Schemes ........................................................ 92

5.5 The Bridged FE-MD Method.............................................................................. 93

5.5.1 Formulation of Stiffness Equations ......................................................... 93

5.5.2 Mapping PBC Nodal Displacements to Primary Cell Nodal Displacements 
5.5.3 Boundary Conditions ...........................................................................97

5.6 Code for Bridged FE-MD Method...................................................................... 97

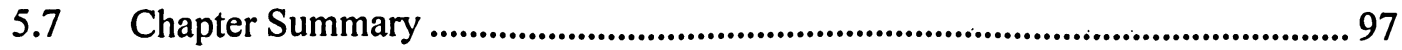

6. APPLICATION OF BRIDGED FE-MD METHOD ...........................................99

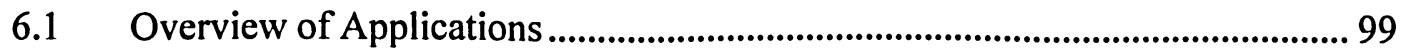

6.2 Application 1: Elastically Stressed Infinite, Perfectly Cylindrical Void in a

Perfect Copper Crystal Lattice .................................................................... 99

6.3 Application 2: Elastically Stressed Infinite, Semi-Cylindrical Notch at the Free

Surface of a Perfect Copper Crystal Lattice ................................................ 103

6.4 Application 3: Elastically Stressed Infinite, Elliptical Void in a Perfect Copper

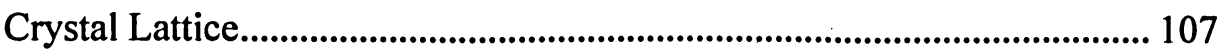

6.5 Discussion and Chapter Summary ........................................................................ 115

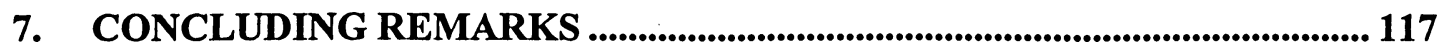

APPENDIX A: PROGRAM EXECUTION FLOWCHARTS FOR CUSTOM MOLECULAR DYNAMICS (MD) CODE................................................. 119

APPENDIX B: INITIAL AND FINAL ENTRIES IN OUTPUT FILE FOR

MD_V_7_00 TEST CASE (MOMENTUM AND ENERGY CONSERVATION FOR SINGLE UNIT CELL) .................................. 125

APPENDIX C: FLOWCHART FOR PROPOSED NUMERICAL EMBEDDED ATOM METHOD (EAM) INTERATOMIC POTENTIAL FITTING PROCEDURE................................................................................................ 127

APPENDIX D: A BRIEF OVERVIEW OF QUANTUM MECHANICS ................. 131 APPENDIX E: CLASSICAL STATISTICAL MECHANICS AND THE MAXWELL-BOLTZMANN DISTRIBUTION LAW........................... 171

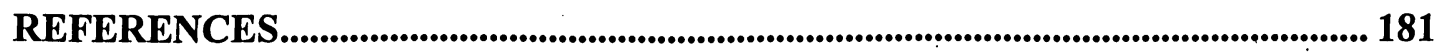




\section{LIST OF TABLES}

Table 1 - Calculated Equilibrium Lattice Constants of Copper at Various Temperatures

Table 2 - Isothermal Young's Modulus and Poisson's Ratio of Copper at Various

Temperatures for $100 \mathrm{MPa}$ Uniaxial Stress (calculated using custom MD

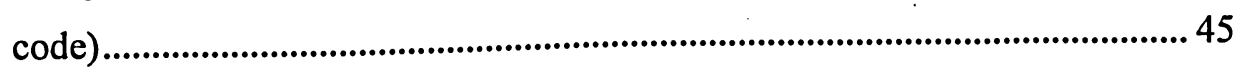

Table 3 - Anisotropic Mechanical Properties of Single Crystal Copper at 293K............. 47

Table 4 - Summary and Comparison of Results for Application 1.................................. 103

Table 5 - Summary and Comparison of Results for Application 2.................................. 107

Table 6 - Summary and Comparison of Results for Application 3 (100 MPa XX FarField Stress) ................................................................................................ 114

Table 7 - Summary and Comparison of Results for Application 3 (100 MPa YY FarField Stress) 


\section{LIST OF FIGURES}

Figure 1 - Two-Dimensional Periodic Boundary Condition ............................................... 21

Figure 2 - General Form of the Lennard-Jones Pair Potential ........................................... 29

Figure 3 - Electron Density of Copper Atom (WinGAMESS Output)............................... 35

Figure 4 - Lennard-Jones Pair Potential for Copper EAM Potential .................................. 36

Figure 5 - Embedding Function for Copper EAM Potential............................................... 36

Figure 6 - Convention for Shear Strain Applicătion in Custom MD Code........................ 42

Figure 7 - Variation of Young's Modulus with Temperature (up to 300K) ...................... 46

Figure 8 - Variation of Poisson's Ratio with Temperature (up to 300K) .......................... 46

Figure 9 - Copper Nano-Layer with Cylindrical Void (View 1; Angstroms).................... 51

Figure 10 - Copper Nano-Layer with Cylindrical Void (View 2; Angstroms)................. 52

Figure 11 - Application 1: XX Stress Increment (MPa) .................................................... 54

Figure 12 - Application 1: XY (YX) Stress Increment (MPa) ........................................... 55

Figure 13 - Application 1: XZ (ZX) Stress Increment (MPa)............................................ 55

Figure 14 - Application 1: YY Stress Increment (MPa) ……............................................ 56

Figure 15 - Application 1: YZ (ZY) Stress Increment (MPa)........................................... 56

Figure 16 - Application 1: ZZ Stress Increment (MPa) ...................................................5 57

Figure 17 - Copper Nano-Layer with Surface Notch (View 1; Angstroms)..................... 59

Figure 18 - Copper Nano-Layer with Surface Notch (View 2; Angstroms) ...................... 59

Figure 19 - Application 2: XX Stress Increment (MPa) .................................................... 62

Figure 20 - Application 2: XY (YX) Stress Increment (MPa)............................................ 62

Figure 21 - Application 2: XZ (ZX) Stress Increment (MPa).......................................... 63

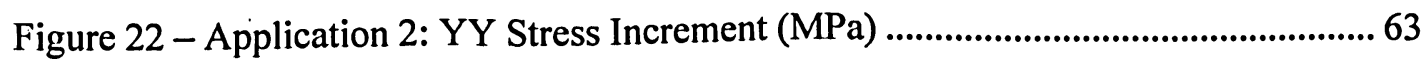

Figure 23 - Application 2: YZ (ZY) Stress Increment (MPa)............................................ 64

Figure 24 - Application 2: ZZ Stress Increment (MPa) ...................................................64

Figure 25 - Copper Nano-Layer with Elliptical Void (View 1; Angstroms) ..................... 66

Figure 26 - Copper Nano-Layer with Elliptical Void (View 2; Angstroms) ..................... 67

Figure 27 - Application 3: XX Stress Increment (MPa) for 100MPa XX Applied Far-

Field Stress. 
Figure 28 - Application 3: XY (YX) Stress Increment (MPa) for 100MPa XX Applied Far-Field Stress

Figure 29 - Application 3: XZ (ZX) Stress Increment (MPa) for 100MPa XX Applied Far-Field Stress

Figure 30 - Application 3: YY Stress Increment (MPa) for 100MPa XX Applied Far-

Field Stress.

Figure 31 - Application 3: YZ (ZY) Stress Increment (MPa) for 100MPa XX Applied

Far-Field Stress.

Figure 32 - Application 3: ZZ Stress Increment (MPa) for 100MPa XX Applied Far-

Field Stress. 72

Figure 33 - Application 3: XX Stress Increment (MPa) for 100MPa YY Applied Far-

Field Stress. 72

Figure 34 - Application 3: XY (YX) Stress Increment (MPa) for 100MPa YY Applied Far-Field Stress

Figure 35 - Application 3: XZ (ZX) Stress Increment (MPa) for 100MPa YY Applied

Far-Field Stress. 73

Figure 36-Application 3: YY Stress Increment (MPa) for 100MPa YY Applied Far-

Field Stress

Figure 37-Application 3: YZ (ZY) Stress Increment (MPa) for 100MPa YY Applied Far-Field Stress

Figure 38 - Application 3: ZZ Stress Increment (MPa) for 100MPa YY Applied Far-

Field Stress 75

Figure 39 - Two-Node Spring Element (for illustration of general stiffness matrix formulation) .78

Figure 40 - Assembly of Linear Spring Elements (for illustration of the effects of PBC) 82

Figure 41 - Assembly of Linear Spring Elements (for illustration of the effects of PBCs) 85

Figure 42 - Proposed Element Discretization (only element 1 shown).............................. 88

Figure 43 - Application 1: XX Stress Increment from FE-MD Bridged Simulation (MPa) 
Figure 44-Application 1: XY (YX) Stress Increment from FE-MD Bridged Simulation (MPa) 100

Figure 45-Application 1: XZ (ZX) Stress Increment from FE-MD Bridged Simulation (MPa) 101

Figure 46 - Application 1: YY Stress Increment from FE-MD Bridged Simulation (MPa) 101

Figure 47-Application 1: YZ (ZY) Stress Increment from FE-MD Bridged Simulation (MPa) 102

Figure 48 - Application 1: ZZ Stress Increment from FE-MD Bridged Simulation (MPa) 102

Figure 49-Application 2: XX Stress Increment from FE-MD Bridged Simulation (MPa) 104

Figure 50-Application 2: XY (YX) Stress Increment from FE-MD Bridged Simulation (MPa) 104

Figure 51 - Application 2: XZ (ZX) Stress Increment from FE-MD Bridged Simulation (MPa) 105

Figure 52 - Application 2: YY Stress Increment from FE-MD Bridged Simulation (MPa) 105

Figure 53 - Application 2: YZ (ZY) Stress Increment from FE-MD Bridged Simulation (MPa) 106

Figure 54 - Application 2: ZZ Stress Increment from FE-MD Bridged Simulation (MPa) 106

Figure 55 - Application 3: XX Stress Increment from FE-MD Bridged Simulation (MPa) for 100MPa XX Applied Far-Field Stress 108

Figure 56-Application 3: XY (YX) Stress Increment from FE-MD Bridged Simulation (MPa) for 100MPa XX Applied Far-Field Stress. 108

Figure 57 - Application 3: XZ (ZX) Stress Increment from FE-MD Bridged Simulation (MPa) for 100MPa XX Applied Far-Field Stress. 109

Figure 58 - Application 3: YY Stress Increment from FE-MD Bridged Simulation (MPa) for 100MPa XX Applied Far-Field Stress 109 
Figure 59 - Application 3: YZ (ZY) Stress Increment from FE-MD Bridged Simulation (MPa) for 100MPa XX Applied Far-Field Stress. 110

Figure 60 - Application 3: ZZ Stress Increment from FE-MD Bridged Simulation (MPa)

for 100MPa XX Applied Far-Field Stress .............................................. 110

Figure 61 - Application 3: XX Stress Increment from FE-MD Bridged Simulation (MPa)

for 100MPa YY Applied Far-Field Stress ............................................... 111

Figure 62-Application 3: XY (YX) Stress Increment from FE-MD Bridged Simulation

(MPa) for 100MPa YY Applied Far-Field Stress ..................................... 111

Figure 63-Application 3: XZ (ZX) Stress Increment from FE-MD Bridged Simulation

(MPa) for 100MPa YY Applied Far-Field Stress ................................... 112

Figure 64 - Application 3: YY Stress Increment from FE-MD Bridged Simulation (MPa)

for 100MPa YY Applied Far-Field Stress ............................................... 112

Figure 65 - Application 3: YZ (ZY) Stress Increment from FE-MD Bridged Simulation

(MPa) for 100MPa YY Applied Far-Field Stress ................................... 113

Figure 66 - Application 3: ZZ Stress Increment from FE-MD Bridged Simulation (MPa)

for 100MPa YY Applied Far-Field Stress ................................................ 113

Figure 67 - Program Execution Flowchart for Master MD Function MD_V_7_00 ...... 120

Figure 68 - Program Execution Flowchart for Function FCC_Gen_V_7_00 and

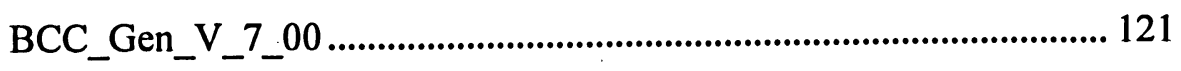

Figure 69 - Program Execution Flowchart for Function CRACK_VOID_V_7_00 ...... 122

Figure 70 - Program Execution Flowchart for Function EAM_Range_V_7_00............ 123

Figure 71 - Program Execution Flowchart for Function EAM_Poly_Fit_V_7_00 ....... 124

Figure 72 - Flowchart for Proposed Numerical EAM Potential Fitting Procedure (part 1;

continued in Figure 73)........................................................................... 128

Figure 73 - Flowchart for Proposed Numerical EAM Potential Fitting Procedure (part 2;

continued from Figure 72) ....................................................................... 129 


\section{English Symbols}

\begin{tabular}{|c|c|c|}
\hline$a$ & : & Lattice constant of crystal \\
\hline$a_{x}(t)$ & : & $\mathrm{X}$ component of acceleration of an atom at time $t$ \\
\hline$B$ & : & Bulk modulus of crystal lattice \\
\hline$c_{11}, c_{12}, c_{44}$ & : & Single crystal cubic elastic constants \\
\hline$d, \partial$ & $:$ & Differential operators \\
\hline$E$ & $:$ & Young's modulus \\
\hline$\vec{F}_{i}$ & : & EAM force vector on atom $i$ \\
\hline$F_{L J}$ & $:$ & Lennard-Jones force \\
\hline$\vec{f}_{i j}$ & : & Force vector on atom $i$ due to atom $j$ \\
\hline$f(v)$ & $:$ & Gaussian probablility density of velocity $v$ \\
\hline$G$ & : & Shear modulus \\
\hline$i, j$ & : & Atom label \\
\hline$k$ & : & Spring stiffness coefficient \\
\hline$k_{B}$ & : & Boltzmann's constant \\
\hline$K$ & : & Finite Element stiffness matrix \\
\hline$L J \_1$ & $:$ & Modified Lennard-Jones potential exponent 1 \\
\hline$L J \_2$ & : & Modified Lennard-Jones potential exponent 2 \\
\hline$m_{i}$ & : & Mass of atom $i$ \\
\hline$N$ & : & Total number of atoms in sphere of influence of active atom \\
\hline$N_{\text {tot }}$ & : & Total number of atoms in model/system \\
\hline$p, q$ & : & General system degrees of freedom \\
\hline$P$ & : & Hydrostatic pressure on crystal lattice \\
\hline$R, r$ & : & General interatomic distance \\
\hline
\end{tabular}




$\begin{array}{lll}\vec{r}_{i} & : & \text { Absolute position vector of atom } i \\ \vec{r}_{j} & : & \text { Absolute position vector of atom } j \\ \vec{r}_{i j} & : & \text { Position vector of atom } i \text { relative to atom } j \\ \dot{\vec{r}}_{i} & : & \text { Velocity vector of atom } i \\ r_{i j} & : & \text { Distance between atom } i \text { and atom } j \\ T & : & \text { Absolute temperature of crystal lattice } \\ U_{E A M} & : & \text { EAM potential energy } \\ U_{L J} & : & \text { Lennard-Jones potential energy } \\ V & : & \text { Volume of domain for virial stress calculation } \\ v_{x}(t) & : & \text { X component of velocity of an atom at time } t \\ x(t) & : & \text { X coordinate of an atom at time } t\end{array}$

\section{Greek Symbols}

$\begin{array}{lll}\varepsilon & : & \text { Lennard-Jones potential parameter defining potential well depth } \\ \varepsilon_{i j} & : & i^{\text {th }} \text { component of engineering strain tensor } \\ \underline{\varepsilon} & : & \text { Engineering strain tensor } \\ \mu & : & \text { Temperature parameter for thermal stress } \\ v & : & \text { Poisson's ratio } \\ \rho\left(r_{i j}\right) & : & \text { Electron density at location of atom } i \text { due to atom } j \\ \rho_{t o t, i} & : & \text { Total electron density at location of atom } i \\ \sigma & : & \text { Lennard-Jones potential parameter defining potential well location } \\ \sigma_{i j} & : & i^{\text {th }} \text { component of virial stress tensor } \\ \sigma & : & \text { Externally applied virial stress tensor } \\ \phi\left(r_{i j}\right) & : & \text { EAM pair potential of atoms } i \text { and } j \\ \Psi & : & \text { EAM embedding function } \\ \varphi\left(r_{i j}\right) & : & \text { General pair potential function } \\ \vec{\nabla} & : & \text { Gradient of a scalar field }\end{array}$




\section{LIST OF ACRONYMS}

\begin{tabular}{lll}
\hline BCC & $:$ & Body Centred Cubic \\
DFT & $:$ & Density Functional Theory \\
EAM & $:$ & Embedded Atom Method \\
FCC & $:$ & Face Centred Cubic \\
FE & $:$ & Finite Element \\
FEA & $:$ & Finite Element Analysis \\
FEM & $:$ & Finite Element Method \\
HF SCF & $:$ & Hartree-Fock Self-Consistent-Field \\
LHS & $:$ & Left Hand Side \\
LJ & $:$ & Lennard-Jones \\
MD & $:$ & Molecular Dynamics \\
PBC & $:$ & Periodic Boundary Condition \\
RHS & $:$ & Right Hand Side
\end{tabular}




\section{LIST OF UNITS}

\begin{tabular}{lll}
\hline & & \\
$\mathrm{amu}$ & $:$ & Atomic Mass Unit \\
$\AA$ & $:$ & Angstroms \\
$\mathrm{C}$ & $:$ & Coulomb \\
$\mathrm{eV}$ & $:$ & Electron volt \\
$\mathrm{GPa}$ & $:$ & Giga Pascal \\
$\mathrm{J}$ & $:$ & Joules \\
$\mathrm{K}$ & $:$ & Degree Kelvin \\
$\mathrm{m}$ & $:$ & Metre \\
$\mathrm{MPa}$ & $:$ & Mega Pascal \\
$\mathrm{N}$ & $:$ & Newton \\
$\mathrm{Pa}$ & $:$ & Pascal \\
$\mathrm{s}$ & $:$ & Second
\end{tabular}




\section{INTRODUCTION}

Nanotechnology is a growing and exciting field today: it promises to advance humanity at an unprecedented rate. It has found its niche in several areas of science including biochemistry, materials science and nano-mechanisms. Nanotechnology deals with phenomena and mechanisms at the atomic level. The main idea behind nanotechnology is that since atoms are the building block of matter for all practical purposes (the constituents of atoms like protons, electrons and neutrons cannot be manipulated as easily as entire atom can be), then studying and manipulating atoms will allow the tailoring of technology to suit mankind's needs. As evident, the nature of nanotechnology research makes experimental equipment extremely expensive to procure. For example, direct observation of atoms requires an electron microscope, which is a very expensive piece of equipment.

Due to the prohibitive costs of experimental equipment for nanotechnology, recent focus has shifted to using computers for analysis of nanotechnology and nanomechanisms. Modern computers are powerful and cost-effective for design and analysis. The key computational tool for nanotechnology is Molecular Dynamics (MD). In the short time since its practical implementation, MD has become invaluable for simulating atoms and molecules. It has become the cornerstone of several researches in nanotechnology. Thanks to the rapid evolution of computers, it has become possible to simulate several millions of atoms using MD. MD is currently being used to simulate proteins in nature, to study fatigue and fracture of metals, to study elastic wave propagation in solids, and to study chemical reactions, among other things. Some specific examples of its application are: studying thermal and mechanical properties of crystalline and amorphous silicon [1,2]; studying brittle fracture in silica [3]; studying mechanical properties of nanocrystalline pure metals and alloys [4,5,6]; studying deposition of metallic and non-metallic atoms onto thin films [7,8]; generating plausible crystal structures for molecular solids [9]; and studying weak interactions between molecules of 
liquids [10]. Recent efforts have been dedicated to improving MD by using advanced atomic concepts $[11,12,13]$.

This thesis focuses on the application of MD to studying the behaviour of perfect metal crystals at the atomic level. As mentioned earlier, the cost of experimental equipment was prohibitive enough to limit the research work to computational analysis. It was recognized from the beginning that similar work in the literature might simply be extended directly into applications on perfect metal crystals. However, use of MD in nanotechnology is a relatively new endeavour at several institutions across the country with only a handful of research groups having knowledge of and access to MD software. Hence it was necessary to go through the exercise of creating custom MD software from scratch to grasp the key concepts of the subject and to have a fully customizable code for the research group. Chapter 2 of this report is therefore dedicated to MD, and presents the MD software programmed by the author for this thesis.

The key to simulating any system with MD is an accurate model of the forces between the constituent atoms or molecules. Almost all these interactive forces are conservative in nature, which means that they can be derived from a scalar potential function. The potential function is usually derived from quantum mechanics, the branch of science that is used to deal with atomic and subatomic particles. Once the potential function is derived, the inter-atomic forces can be calculated as the spatial gradient of the potential. These forces are used to formulate the dynamic equations of motion of the system, which are then integrated by the MD software to yield the trajectories of atoms and molecules. The Embedded Atom Method (EAM) potential is one such inter-atomic potential that has proven to be very accurate for metals. EAM potentials have been developed for a handful of metals by various researchers [19]. However, for the same reasons that prompted the development of a custom MD software, it was decided to develop a numerical fitting procedure to produce EAM potentials for cubic metals. The procedure would enable the research group to easily develop EAM potentials from scratch for metals whose EAM potentials are not readily available. Chapter 3 of this report is dedicated to the EAM potential, and presents a numerical fitting procedure proposed by the author for generating EAM inter-atomic potentials for pure Face Centred Cubic (FCC) and Body Centred Cubic (BCC) metals. Chapter 4 then demonstrates the 
application of MD and the EAM potential for determining virial stress increment patterns in perfect copper crystal lattices with voids of definite geometries.

For MD to be effective, the simulation has to be conducted for a substantial number of time steps (i.e. the equations of motion must be integrated for a total time that is substantial enough to yield reliable results). This is sometimes time-consuming, even with powerful computers. To overcome this, certain principles of MD can be combined with the Finite Element (FE) method to yield bridged FE-MD methods that are much more efficient than MD simulation, and yet yield comparable results. Recent research has been geared towards developing such hybrid methods [14]. Chapter 5 presents one such bridged FE-MD method that implements a novel element discretization scheme proposed by the author. Chapter 6 finally concludes the thesis by comparing results from the bridged FE-MD method with results from MD simulation alone. 


\section{MOLECULAR DYNAMICS}

\subsection{Introduction to Molecular Dynamics}

Molecular Dynamics (MD) is a computational method used to directly simulate the dynamic behaviour of atoms and molecules. The foundation of any MD code is an efficient N-body dynamics simulator, which integrates the dynamic equations of motion of the system to determine the trajectory of atoms and molecules in a system. Due to advances in computer power, MD can now be used to simulate millions of atoms over substantial time periods (substantial relative to atomic motion; time periods of 1 nanosecond to 1 microsecond are substantial at this scale of natural phenomena). MD is the only viable method of simulating a large number of atoms or molecules interacting with each other. This is an important advantage that MD has over numerical quantum mechanical simulation methods, which are limited in the size of the system they can simulate. That being said, a few words on these numerical quantum mechanical simulation methods are in order.

There is no doubt today that the behaviour of atoms and subatomic particles is governed by the laws of quantum mechanics. Quantum mechanics has given us insight into some of the most challenging problems in physics. A very brief overview of quantum mechanics is given in Appendix D. It suffices to say here that exact solutions to the quantum mechanical differential equation (the Schrödinger wave equation) are obtainable only for the simplest of atoms like the Hydrogen or Hydrogen-like atoms. For all other purposes, various numerical methods have been developed to solve the Schrödinger wave equation approximately. The Hartree-Fock Self-Consistent-Field (HF SCF) method is an example of one such numerical method (see Appendix D for a discussion on the HF SCF method). However, even these numerical methods in quantum mechanics are restricted to systems containing a relatively small number of atoms or molecules. MD, on the other hand, incorporates the fundamental results from quantum mechanic simulations or studies to simulate a large number of atoms. 
The most important fundamental result from quantum mechanics is a model of the inter-atomic forces, which are required by the dynamic equations of motion for calculating atomic trajectories. These inter-atomic forces are conservative forces and hence they can be represented by a scalar potential function. There are a number of interatomic potential functions in use today, some examples of which are: the Lennard-Jones pair potential for gases, liquids and some solids; the Tersoff potential for Silicon; and the Embedded Atom Method (EAM) multi-body potential for metals. Once the forces are modelled from quantum mechanics, the trajectories of atoms and molecules can be calculated using MD.

MD has become an invaluable tool in studying material behaviour at the atomic level. It has been used to study metal fracture, elastic wave propagation, bending of carbon nano-tubes, etc. The importance of MD in nanotechnology is epitomized by the world's fastest parallel computing system, MDGrape- $3^{*}$, which is a petaflop $(1 \mathrm{e}+15$ floating point operations per second) capable system designed exclusively for performing MD simulations on proteins.

The basic premise and use of MD has been presented. The following section introduces the inter-atomic potential most commonly used for metals in MD simulations: the Embedded Atom Method (EAM) potential.

\subsection{The Embedded Atom Method Inter-Atomic Potential for Metals}

The Embedded Atom Method (EAM) potential is one of the most accurate inter-atomic potential functions for single-crystal metal lattices. It is based on Density Functional Theory (DFT), a quantum mechanical theory that states that the electron density of any system of atoms determines all its ground-state properties $[15,16]$.

A single-crystal metal lattice consists of positively charged nuclei embedded in a 'sea' of valence electrons. The electron 'sea' or cloud binds the nuclei together and gives the crystal lattice its stability. Since the arrangement of the nuclei is periodic in all

\footnotetext{
- High Performance Molecular Simulation Team, Computational \& Experimental Systems Biology Group, Genomic Sciences Center, RIKEN (http://mdgrape.gsc.riken.jp/)
} 
direction in a perfect metal lattice, the inter-metallic bond is non-directional, and the potential function need not have terms related to bond angles.

The basic interaction between the nuclei is modelled as a pair potential (e.g. Coulomb electrostatic interaction, Lennard-Jones potential interaction or a Morse potential interaction). Pair potential functions and their gradients (i.e. the forces associated with them) can be individually calculated and finally summed to yield the total potential energy of the system. That means that for any pair potential $\varphi\left(r_{i j}\right)$ which is a function of the scalar distance between atoms $i$ and $j$ (i.e. $r_{i j}$ ), the following expressions are valid:

$$
\begin{gathered}
\varphi_{i}=\sum_{\substack{j=1 \\
j \neq 1}}^{N} \varphi_{i j} \\
-\vec{\nabla} \varphi_{i}=-\sum_{\substack{j=1 \\
j \neq i}}^{N} \vec{\nabla} \varphi_{i j}
\end{gathered}
$$

The remaining cohesive force contributing to anharmonic crystal behaviour is due to the 'sea' of valence electrons and therefore is a function of the electron density at a point in the lattice. However, since electron density at a point depends on all nuclei in the vicinity, the potential energy from this electron density is not a simple pair potential, but is a multi-body potential. These kinds of potentials generally do not obey equations (1) and (2).

The EAM potential, which was proposed by Daw and Baskes $[17,18]$ and developed for a handful of metals by Foiles, Baskes and Daw [19], combines a pair potential (for the basic interaction amongst the nuclei) with a multi-body potential (for anharmonic interactions between the nuclei and the electron cloud) to give the total potential energy of the metal crystal lattice. The EAM potential energy of a system of $N_{\text {tot }}$ atoms in a metal lattice, each of which is affected (or influenced) by $N$ surrounding atoms, is given by the following expression: 


$$
U_{t o t, E A M}=\sum_{i=1}^{N_{t o n}}\left[U_{i, E A M}\right]=\sum_{i=1}^{N_{t o n}}\left[\frac{1}{2} \sum_{\substack{j=1 \\ j \neq i}}^{N} \phi\left(r_{i j}\right)+\Psi\left(\rho_{t o t, i}\right)\right]
$$

In equation (3), $\phi\left(r_{i j}\right)$ is the pair potential and $\Psi$ is the energy required to 'embed' a nucleus in the surrounding electron cloud. $\Psi$ is appropriately called the 'embedding function' and is a function of the total electron density $\rho_{t o t, i}$ at the location of the nucleus $i$, as evident in the last term of equation (3). It is this term that distinguishes the EAM potential from pair potentials and gives EAM its ability to capture metallic behaviour accurately. The total electron density at the location of nucleus $i$ is further approximated as the scalar sum of atomic electron densities of the $N$ atoms surrounding nucleus (or atom) $i$. Hence equation (3) is rewritten as:

$$
U_{t o t, E A M}=\sum_{i=1}^{N_{t o r}}\left[\frac{1}{2} \sum_{\substack{j=1 \\ j \neq i}}^{N} \phi\left(r_{i j}\right)+\Psi\left\{\sum_{\substack{j=1 \\ j \neq i}}^{N} \rho\left(r_{i j}\right)\right\}\right]
$$

The force due to a potential at a point is the spatial gradient of the total system potential energy at that point. The same definition applies to the EAM potential. The spatial gradient of equation (4) for an atom $i$ gives the following expression for the force on that atom [20].

$$
\vec{F}_{i}=-\sum_{\substack{j=1 \\ j \neq i}}^{N}\left[\frac{d \phi\left(r_{i j}\right)}{d r_{i j}}+\left(\frac{d \Psi}{d \rho_{i, t o t}}+\frac{d \Psi}{d \rho_{j, t o t}}\right)\left(\frac{d \rho\left(r_{i j}\right)}{d r_{i j}}\right)\right] \frac{\vec{r}_{i j}}{\left|\vec{r}_{i j}\right|}, \quad 1 \leq i \leq N_{t o t}
$$

\subsection{The General Molecular Dynamics Algorithm}

The purpose of Molecular Dynamics (MD) is to calculate the trajectory of atoms in phase-space by integrating the dynamic equations of motion for each atom. Phase-space is the $\mathrm{N}$-dimensional space, a point of which completely describes the position and 
momentum of an atom. For the case of atoms in a plane (two-dimensional case), the position part of phase-space (called configuration-space) is two-dimensional with the $\mathrm{X}$ and Y Cartesian coordinates along the two axes. The momentum part of phase-space (called momentum-space) is also two-dimensional with the $\mathrm{X}$ and $\mathrm{Y}$ momentum components of each atom along the two axes. That makes the phase-space of a twodimensional case four-dimensional. Similarly, the phase-space of the general, threedimensional case is six-dimensional (a three-dimensional configuration-space and a three-dimensional momentum-space). The dynamic equations of motion require the forces on each atom to be evaluated using a suitable inter-atomic potential (which is usually modelled using quantum mechanics, as discussed in the preceding sections).

Taking all these facts into account, the general MD algorithm when using Newton's laws of motion can be written as follows (information here is put together from various sections of a MD reference book [21]):

1. Obtain positions and momentum (i.e. velocities, indirectly) of all the atoms at time $t_{i}$.

2. Evaluate the forces on each and every atom at that same instant of time, $t_{i}$.

3. Calculate the acceleration of each and every atom by dividing the force on an atom by its mass (it is being assumed here that the atoms are point masses; this is a very reasonable assumption since the size of the atomic nucleus, where almost all the mass of the atom is concentrated, is less than a thousandth of the inter-atomic distance, even in tightly packed solids).

4. Choose a very small time interval $\Delta t$ over which the time integration will be performed.

5. Integrate the dynamic equations of motion over this time interval by any numerical method. One direct approach is to:

a) Integrate the acceleration first to get the velocity increment.

b) Add the velocity increment to the velocity of that atom at time $t_{i}$. This is the velocity of the same atom at time $t_{i}+\Delta t$.

c) Integrate the velocity to get the position increment. 
d) Add the position increment to the position of that atom at time $t_{i}$. This is the position of the same atom at time $t_{i}+\Delta t$.

6. Repeat steps 2 through 5 , assuming that $t_{i+1}=t_{i}+\Delta t$ until the required time for the simulation has elapsed.

7. Tabulate atomic positions and momentum (or velocities) at each instant of time.

8. Use tabulated data to determine other quantities of interest, like: average kinetic energy of all the atoms; trajectory of the centre-of-mass of the system; etc.

This is the algorithm for almost all MD codes in existence today. The differences between the codes are simply in how the dynamic equations are formulated (Newtonian vs. Lagrangian vs. Hamiltonian equations) and how they are integrated numerically (Taylor series approximation; predictor-corrector methods like Gear's algorithm; RungeKutta integration; etc.).

\subsection{Custom Molecular Dynamics Code MD_V_7_o0}

There is MD freeware available on the World Wide Web for general usage $\left(\mathrm{XMD}^{\dagger}\right.$ is one such MD freeware). However, for this thesis project, it was decided to write a custom MD code since a major aim of the thesis was to develop a code for fitting the EAM interatomic potential for various metals (Chapter 3), which required specialization of an MD code. Making changes to the freeware source code was found to be very difficult due to non-familiarity with the source code.

The custom MD code is written in MATLAB ${ }^{\mathrm{TM} \ddagger}$. MATLAB ${ }^{\mathrm{TM}}$ has an excellent set of library functions that handle all sorts of mathematical tasks, from table interpolation, to polynomial fitting, to extracting numerical data from text files.

The following sections present details of the author's custom MD code, along with the supporting theory.

\footnotetext{
${ }^{\dagger}$ Center for Materials Simulation, University of Connecticut Institute of Materials Science (http://www.ims.uconn.edu/centers/simul/)

‡ Developed by The MathWorks ${ }^{\circledR}$ (http://www.mathworks.com/)
} 


\subsubsection{Program Execution Flowcharts}

Figure 67 through Figure 71 in Appendix A show the program execution flowcharts for the author's custom MD code. Each figure corresponds to one particular function in the code.

\subsubsection{Function Descriptions}

\subsubsection{MATLAB ${ }^{\text {TM }}$ Function MD_V_7_00}

MD_V_7_00 is the master MD function that coordinates operations and calculations between all other functions. MD_V_7_00 performs the following intrinsic tasks (i.e. tasks for which it doesn't depend on other functions):

1. Read initial atomic positions and velocities.

2. For each atom, generate an array containing distances to other atoms that are less than the Embedded Atom Method (EAM) potential cut-off distance.

3. Calculate the total force on each atom using the EAM force expression. Use this force to estimate position and velocity after the time interval using a second order Taylor series expansion for position and a first order Taylor series expansion for velocity.

4. Write position and velocity data to a text file at each time step.

5. Use the recorded data to create a movie of the atomic motion during the simulation.

The Taylor series for position estimation is now derived. Let the $\mathrm{X}$ coordinate of a particle at time $t$ be $x(t)$, while the $\mathrm{X}$-direction components of velocity and acceleration of the same particle at the same time $t$ be $v_{x}(t)$ and $a_{x}(t)$ respectively. Then the X coordinate at time $t+\Delta t$ (where $\Delta t$ is the user-defined time interval) can be obtained using the following Taylor series expansion for $x$ about $t$ : 


$$
x(t+\Delta t)=x(t)+\Delta t \frac{d x(t)}{d t}+\frac{(\Delta t)^{2}}{2} \frac{d^{2} x(t)}{d t^{2}}+O\left[(\Delta t)^{3}\right]
$$

This is a second order Taylor series expansion with order of the error given by the last term. Now, the first and second derivatives of $x(t)$ with respect to time are merely the $\mathrm{X}$ direction components of velocity and acceleration respectively. Hence equation (6) can be rewritten as:

$$
x(t+\Delta t) \approx x(t)+\Delta t v_{x}(t)+\frac{\Delta t^{2}}{2} a_{x}(t)
$$

Similar expressions can be written for the Y- and Z- directions. Equation (7) is used to predict the positions of the atoms at time $t+\Delta t$ using the positions, velocities and accelerations at time $t$ (which are all known). It is accurate to the second order of time interval.

To develop an expression for predicting the velocities at time $t+\Delta t$, write the following Taylor series expansion for $v_{x}$ about $t$ :

$$
v_{x}\left(t+\frac{\Delta t}{2}\right)=v_{x}(t)+\frac{\Delta t}{2} \frac{d v_{x}(t)}{d t}+O\left[\left(\frac{\Delta t}{2}\right)^{2}\right]
$$

Obviously, this expansion predicts the velocity at the half-time step. The first derivative of $v_{x}$ with respect to time is the $\mathrm{X}$-direction component of acceleration. Hence equation (8) can be rewritten as follows:

$$
v_{x}\left(t+\frac{\Delta t}{2}\right)=v_{x}(t)+\frac{\Delta t}{2} a_{x}(t)+O\left[\left(\frac{\Delta t}{2}\right)^{2}\right]
$$

A similar backward expansion about $t+\Delta t$ gives the following expression: 


$$
v_{x}\left(t+\frac{\Delta t}{2}\right)=v_{x}(t+\Delta t)-\frac{\Delta t}{2} \frac{d v_{x}(t+\Delta t)}{d t}+O\left[\left(\frac{\Delta t}{2}\right)^{2}\right]
$$

This can again be rewritten as follows:

$$
v_{x}\left(t+\frac{\Delta t}{2}\right)=v_{x}(t+\Delta t)-\frac{\Delta t}{2} a_{x}(t+\Delta t)+O\left[\left(\frac{\Delta t}{2}\right)^{2}\right]
$$

Equating (9) and (11) and solving for $v_{x}(t+\Delta t)$ gives the following final expression for the X-direction component of velocity at time $t+\Delta t$ :

$$
v_{x}(t+\Delta t) \approx v_{x}(t)+\frac{\Delta t}{2}\left[a_{x}(t)+a_{x}(t+\Delta t)\right]
$$

This expression is the popular velocity Verlet algorithm used in many other MD codes, and is more accurate than a regular forward difference evaluation of the velocity about time $t$ because of the $1 / 4$ factor in the error term in equations (9) and (11). Equation (12) requires knowledge of the acceleration at time $t+\Delta t$. Since the positions at time $t+\Delta t$ can be determined using equation (7), it is a simple matter of evaluating the forces at the new locations and dividing by the masses of the atoms.

Force evaluation is probably the most important function that MD_V_7_00 does. The forces are calculated using the spatial derivative of the EAM potential. Equation (4) is used to calculate the EAM potential energy, while equation (5) is used to calculate the force on each atom during the simulation. The three component functions of the EAM potential (the pair potential, the electron density and the embedding function) are represented as either discrete functions in MATLAB ${ }^{\mathrm{TM}}$ (in the case of the pair potential and electron density) or as tabulated data stored as a text file (in the case of the embedding function). 


\subsubsection{MATLAB ${ }^{\mathrm{TM}}$ Functions FCC_Gen_V_7_00 and BCC_Gen_V_7_00}

These functions construct a Face Centred Cubic (FCC) or a Body Centred Cubic (BCC) crystal lattice with user-defined lattice parameters. The functions first generate the lattice and then save the atomic positions in a text file for reading by all other functions. They also initialize atomic velocities according to the following Gaussian probability density function (see Appendix $\mathrm{E}$ for a derivation based on statistical mechanics):

$$
\begin{aligned}
& f\left(v_{x}\right)=\sqrt{\frac{m}{2 \pi k_{B} T}} \exp \left(-\frac{m v_{x}{ }^{2}}{2 k_{B} T}\right) \\
& f\left(v_{y}\right)=\sqrt{\frac{m}{2 \pi k_{B} T}} \exp \left(-\frac{m v_{y}{ }^{2}}{2 k_{B} T}\right) \\
& f\left(v_{z}\right)=\sqrt{\frac{m}{2 \pi k_{B} T}} \exp \left(-\frac{m v_{z}{ }^{2}}{2 k_{B} T}\right)
\end{aligned}
$$

In equation (13), $m$ is the mass of each atom, $k_{B}$ is Boltzmann's constant $(1.3806503 \times$ $\left.10^{-23} \mathrm{~m}^{2} \cdot \mathrm{kg} \cdot \mathrm{s}^{-2} \cdot \mathrm{K}^{-1}\right)$ and $T$ is the initial absolute temperature of the system in Kelvin. The mean velocity of the distribution is zero. Velocities are chosen such that their arithmetic mean is also zero; this ensures that the centre-of-mass of the system has zero initial velocity.

This distribution is governed by the initial temperature of the simulation, as evident in the exponential term in the equation set. The average kinetic energy of atom $i$ is further related to the temperature by the following common relationship (based on the principle of equipartition of energy in statistical mechanics):

$$
K \cdot E_{\text {avg. }, i}=\frac{1}{2} m_{i}\left(\left(v_{x, 1}{ }^{2}\right)_{\text {avg. }}+\left(v_{y, 1}{ }^{2}\right)_{\text {avg. }}+\left(v_{z, 1}{ }^{2}\right)_{\text {avg. }}\right)=\frac{3}{2} k_{B} T
$$

Then the average kinetic energy of the entire system at absolute temperature $T$ is: 


$$
K . E_{\text {avg. }, \text { total }}=\sum_{i=1}^{N_{\text {tor }}} \frac{1}{2} m_{i}\left(\left(v_{x, i}{ }^{2}\right)_{\text {avg. }}+\left(v_{y, i}{ }^{2}\right)_{\text {avg. }}+\left(v_{z, i}{ }^{2}\right)_{\text {avg. }}\right)=\frac{3}{2} N_{\text {tot }} k_{B} T
$$

, where $N_{t o t}$ is the total number of atoms in the system. For initializing the velocity distribution, it is assumed that the average total kinetic energy given by equation (15) corresponds to the instantaneous initial total kinetic energy of the distribution. Hence:

$$
K . E_{\text {total }, \text { initial }}=\sum_{i=1}^{N_{\text {tot }}} \frac{1}{2} m_{i}\left(v_{x, i}{ }^{2}+v_{y, i}{ }^{2}+v_{z, i}{ }^{2}\right) \triangleq K \cdot E_{\text {avg., } \text { total }}=\frac{3}{2} N_{\text {tot }} k_{B} T
$$

The function flowchart is given in Figure 68 (Appendix A).

\subsubsection{MATLAB ${ }^{\mathrm{TM}}$ Functions CRACK_VOID_V_7_00}

This function constructs a void within the crystal lattice generated by FCC_Gen_V_7_00 or BCC_Gen_V_7_00. A void is essentially a three-dimensional space devoid of any atoms, whose surface can theoretically be represented by an equation in threedimensional shape. Practically speaking, this is possible only for simple enclosing surfaces like: the surface of a sphere (for a spherical void); the surface of a cylinder (for a cylindrical void); etc. CRACK_VOID_V_7_00 requires the user to symbolically enter the equation of a residual function (which is possible in MATLAB ${ }^{\mathrm{TM}}$ ) based on the equation of the void's outer surface. The user defines the residual function in such a way that all points within the void yield a non-negative residual value. CRACK_VOID_V_7_00 then calculates the value of the residual function at each atom's position, and discards atoms whose positions yield non-negative residuals. The remaining atoms are the active atoms, and are the only ones used in the MD simulation. Formulation of residual functions is demonstrated during the application of the custom MD code (Chapter 4). The function flowchart is given in Figure 69 (Appendix A). 


\subsubsection{MATLAB ${ }^{\mathrm{TM}}$ Function EAM_Range_V_7_00}

This function scans the EAM potential data text file and returns the embedding function data points (which, as mentioned in Section 2.4.2.1, are stored in the form of a lookup table in the text file). A fourth order spline is fit into these data points by MATLAB ${ }^{\mathrm{TM}}$ function EAM_Poly_Fit_V_7_00 (Section 2.4.2.5) to obtain functional forms of the data. The function flowchart is given in Figure 70 (Appendix A).

\subsubsection{MATLAB ${ }^{\text {TM }}$ Function EAM_Poly_Fit_V_7_00}

This function fits a fourth order spline into the data arrays returned by function EAM_Range_V_7_00. The MATLAB ${ }^{\mathrm{TM}}$ library function spapi $($ is used to calculate the splines. The function also calculates the derivative of the spline using MATLAB ${ }^{\mathrm{TM}}$ library function fnder(). The function flowchart is given in Figure 71 (Appendix A).

\subsubsection{Additional Theory}

\subsubsection{Average Stress Calculation: The Virial Stress}

The average stress due to atoms in a domain of volume $\mathrm{V}$ can be calculated using the well-known virial theorem, and the resulting stress tensor is called the virial stress tensor. The virial theorem was originally put forth by Clausius [22] to describe the relationship between average values of bound properties of the system as the time of averaging becomes infinitely long.

The virial stress of $N$ atoms in a domain of volume $V$ is given by the following expression [23]: 


$$
\underline{\sigma}=-\frac{1}{V} \sum_{i=1}^{N}\left[m_{i} \dot{\vec{r}}_{i} \otimes \dot{\vec{r}}_{i}+\frac{1}{2} \sum_{\substack{j=1 \\ j \neq i}}^{N}\left(\vec{r}_{i j} \otimes \vec{f}_{i j}\right)\right]
$$

The right hand side of this equation contains two parts. The first part depends on the velocity of the atoms and therefore is the thermal stress of the system. The second part depends on inter-atomic forces and therefore is the internal mechanical stress of the system. The sum must be in equilibrium with the externally applied mechanical stress on the system (i.e. with the left-hand-side of equation (17)). In equation (17), $\vec{r}_{i j}$ is the vector from atom $j$ to atom $i$ :

$$
\vec{r}_{i j}=\vec{r}_{i}-\vec{r}_{j}
$$

, $\dot{\vec{r}}_{i}$ is the velocity vector of atom $\mathrm{i}, \vec{f}_{i j}$ is the force exerted on atom $i$ by atom $j$ (calculated using equation (5)), and $m_{i}$ is the mass of atom $i . \otimes$ denotes the Kronecker tensor product of two vectors, defined as:

$$
\vec{a} \otimes \vec{b}=\bar{a} \bar{b}^{T}=\left[\begin{array}{l}
a_{x} \\
a_{y} \\
a_{z}
\end{array}\right]\left[\begin{array}{lll}
b_{x} & b_{y} & b_{z}
\end{array}\right]=\left[\begin{array}{ccc}
a_{x} b_{x} & a_{x} b_{y} & a_{x} b_{z} \\
a_{y} b_{x} & a_{y} b_{y} & a_{y} b_{z} \\
a_{z} b_{x} & a_{z} b_{y} & a_{z} b_{z}
\end{array}\right]
$$

The stress tensor given by equation (17) is a symmetric tensor, with normal stresses given by the diagonal elements and shear stresses given by the off-diagonal elements. In a perfect crystal at any temperature, the shear stresses are expected to be zero while a normal stress is negative (compressive) if the crystal edge-length along that direction is less than the equilibrium lattice constant at that temperature, and positive (tensile) if the crystal edge length is greater than the equilibrium lattice constant at that temperature. However, it must be emphasized that the virial theorem deals with averages over an essentially infinite time. Hence the average stress tensor over a large number of runs will yield zero shear stresses for a perfect unstrained crystal, but the instantaneous shear 
stresses need not be zero because the atoms are continually moving about their equilibrium lattice positions, thereby breaking the symmetry at any instant of time. A special case arises at $0 \mathrm{~K}$, at which atoms do not possess any velocity according to classical theories (note that the phenomenon of zero-point energy, as predicted by quantum mechanics, is not taken into account in the simulations). At absolute zero, the instantaneous shear stresses are all exactly zero because of perfect symmetry in arrangement of atoms at all times.

It has been argued recently [24] that the virial theorem is not an accurate representation of externally applied mechanical stress. However, the virial stress remains the only viable and popular method of calculating stresses for discrete-particle systems, and has been used in atomistic simulations quite successfully ([25], for example). In addition, the virial stress has another powerful feature that makes it attractive for engineering applications: the thermal stress is already accounted for in the virial stress equation (17), as already mentioned. By using a suitable relationship between atomic velocity and temperature (which has already been presented in Section 2.4.2.2), thermal stresses at any required temperature can be readily imposed on the atoms. This is essential for capturing thermal properties of the crystal, like thermal expansion.

\subsubsection{Hydrostatic Pressure and Equilibrium Lattice Constant}

Equation (17) is used in the code for determining the equilibrium lattice constant at a particular temperature, since a metal crystal expands with increase in temperature. During an equilibration run, the lattice constant is adjusted periodically until the average hydrostatic pressure acting on the crystal becomes almost zero. At zero hydrostatic pressure, the crystal is neither in compression nor in tension, and hence is in equilibrium with the surroundings. Note that atmospheric pressure is neglected compared to the internal stresses of the crystal since atmospheric pressure is only slightly greater than 1 Mega Pascal, or $1 \mathrm{MPa}(101,325 \mathrm{~Pa}$ at mean sea level to be precise), while crystal stresses are typically tens or hundreds of $\mathrm{MPa}$.

The hydrostatic pressure acting on the crystal is given by the following equation: 


$$
P=-\left(\frac{\sigma_{x x}+\sigma_{y y}+\sigma_{z z}}{3}\right)
$$

$P$ denotes constant pressure acting on the faces of the crystal and is the negative average of the normal stresses from the stress tensor (17). The negative sign is included because a positive hydrostatic pressure corresponds to a negative hydrostatic stress by convention.

Using the following common definition of the bulk modulus, $B$, of a material:

$$
B=-V \frac{d P}{d V}
$$

, where $V$ is the volume of the material, one can arrive at the following equation for the volume at zero final pressure [26]:

$$
\left.V\right|_{P=0}=V \exp \left(\frac{P}{B}\right)
$$

Here, $V$ is the initial volume of the crystal, while $P$ is the initial hydrostatic pressure as defined by equation (20).

An unstressed FCC or BCC crystal at any temperature can be considered as a cube. Hence $V=a^{3}$, where $a$ is the lattice constant (or edge length) of the crystal. Using this in equation (22) gives the following expression for the equilibrium lattice constant at any temperature:

$$
\left.a\right|_{P=0}=a \exp \left(\frac{P}{3 B}\right)
$$

Equation (23) is used to periodically change the initial lattice constant and loop the simulation until the average lattice constant over a large number of runs converges to the 
desired accuracy. The final average lattice constant is taken to be the equilibrium lattice constant at that particular temperature.

\subsubsection{Engineering Strain Tensor for a Desired Principal Virial Stress Tensor}

The procedure used for determining the equilibrium lattice constant at a specific temperature (given in Section 2.4.3.2) can be modified to enable the determination of a strain tensor that produces a desired principal virial stress tensor. The stress tensor is a principal stress tensor since only desired normal stresses can be obtained using the procedure; the shear stresses will remain zero.

To achieve this, it is sufficient to write an expression similar to equation (23) for each of the three directions of the crystal, with the hydrostatic pressure replaced by the difference between the desired normal stress along each direction and the current (calculated) normal stress along that direction. Hence:

$$
\begin{aligned}
& \left.a_{x}\right|_{\sigma_{x}=\sigma_{r x}}=a_{c x} \exp \left(\frac{\sigma_{r x}-\sigma_{c x}}{3 B}\right) \\
& \left.a_{y}\right|_{\sigma_{y}=\sigma_{r y}}=a_{c y} \exp \left(\frac{\sigma_{r y}-\sigma_{c y}}{3 B}\right) \\
& \left.a_{z}\right|_{\sigma_{z}=\sigma_{r z}}=a_{c z} \exp \left(\frac{\sigma_{r z}-\sigma_{c z}}{3 B}\right)
\end{aligned}
$$

Subscript $r$ denotes the required stress, while subscript $c$ denotes the calculated stress. Equation (24) makes intuitive sense: if the required stress exceeds the calculated stress, then the exponential term is greater than 1 and the lattice size is increased (i.e. the normal strain is increased); if the required stress is less than the calculated stress, then the exponential term is lesser than 1 and greater than 0 and the lattice size is decreased (i.e. the normal strain is decreased). Note that all three components of stress in equation (24) are not independent of each other: a change of lattice size in one direction generally affects all components of the EAM force on the atoms (as per equation (5)), which in turn influences the normal stresses in the other directions also (as per equation (17)). Hence 
the simulation is looped while periodically applying equation (24) until all three lattice sizes (i.e. $a_{x}, a_{y}$ and $a_{z}$ ) converge to the desired accuracy.

\subsubsection{Periodic Boundary Condition in Molecular Dynamics}

It is impossible to simulate a large piece of metal using molecular dynamics because there are simply too many atoms (billions to trillions) for any modern computer to handle. Hence MD is used to simulate a few unit cells of the crystal, with the surrounding unit cells being represented using Periodic Boundary Condition (PBC). When PBC is used, exact replicas of the active unit cell (called the primary cell) are made in the direction of PBC activation. These replicas are called image cells. The positions of atoms in the image cells are usually obtained by simple translation of the positions of the corresponding atoms in the primary cell. The velocities of atoms in the image cells are usually set exactly equal to the velocities of the corresponding atoms in the primary cell. Figure 1 shows the concept of PBC for a two-dimensional case ${ }^{\S}$. The central shaded cell is the primary cell, while all other cells are the image cells.

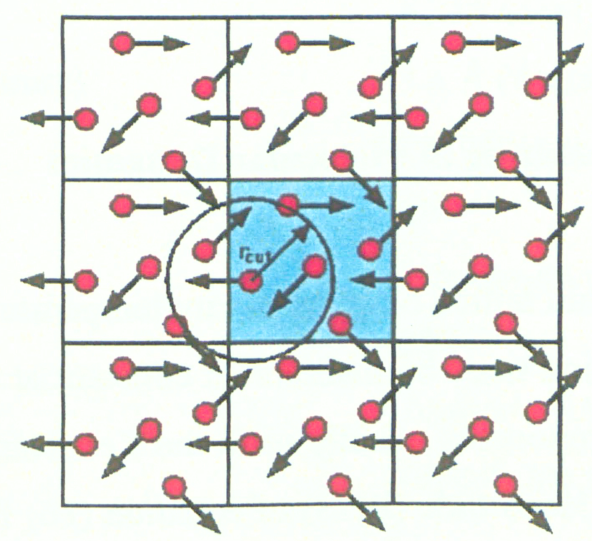

Figure 1 - Two-Dimensional Periodic Boundary Condition

\footnotetext{
${ }^{\S} \mathrm{http}: / /$ www.compsoc.man.ac.uk/ lucky/Democritus/Theory/pbc-mi.html
} 
The biggest advantage of using $\mathrm{PBC}$ is that the effect of the surrounding crystal is captured quite well. This is important to keep the atoms oscillating about their equilibrium lattice positions. Otherwise, a single unit cell simulated by itself would either expand to a very large volume or collapse into a very small volume. The important question then is just how many image cells are needed. The answer lies in the potential function: the number of image cells in each direction is set such that the maximum distance between an atom in the primary cell and any atom in any image cell just exceeds the cut-off distance for that potential. The cut-off distance is the distance beyond which the potential becomes negligible and can be taken as zero for all practical purposes. In typical simulations, this translates to one to three image cells in each direction.

The author's MD code implements $\mathrm{PBC}$ in a slightly different way compared to the afore-mentioned description. The atoms in the image cells are assumed to be fixed with zero velocity at their equilibrium lattice positions. This was required to ensure that the primary cell was stable and did not distort unusually. The immediate disadvantage of this is that momentum of the system will not be conserved, since external forces are required to restrain the image cell atoms from moving. However, the primary unit cell does not move, which is all that is required from the MD simulation. Hence this is not as much of a problem as it might seem to be.

\subsubsection{Temperature Clamping in Molecular Dynamics}

To determine the equilibrium lattice constant at any temperature using equation (23), the temperature of the simulation must be maintained constant at all times. This is achieved by scaling the velocity components equally until the Left Hand Side (LHS) of equation (16) agrees with the Right Hand Side (RHS) of equation (16) for a temperature $T$. This is a very simple procedure, and will not be discussed in further detail. 


\subsection{Testing of Custom Molecular Dynamics Code: Momentum and Energy Conservation for Single Unit Cell (No Periodic Boundary Condition)}

The first test case tests how well MD_V_7_00 conserves system momentum and energy when $P B C$ is not used. Because all the forces are conservative (i.e. are derived from a potential function) and are internal, the total energy (potential plus kinetic) and momentum of the system must be constant at all times. To check momentum conservation, the velocity of the centre-of-mass of the system (which is always initialized as zero) is monitored throughout the run (using the output text file generated by the code). The total energy in the output text file is also monitored to ensure that it remains more or less constant. Typically, the momentum is exactly conserved, while energy very slightly oscillates about the ideal total energy because of numerical inaccuracies.

A single FCC unit cell of copper was used for the simulation. Important parameters for the simulation are as follows:

Material

Atomic mass

Initial lattice constant (arbitrary)

Initial temperature

Temperature clamp

Time interval

Time steps

Interatomic Potential
: $\quad$ Copper

: $\quad 63.55 \mathrm{amu}$ (Atomic Mass Unit)

: $\quad 3.4 \AA$ (Angstroms)

: $\quad$ OK (since no $\mathrm{PBC}$ is used)

: Off

: $\quad 1$ femto-second $\left(10^{-15} \mathrm{sec}\right)$

: $\quad 250$

: Author's EAM potential for copper

(details are presented in Chapter 3)

The above parameters are entered into the MD_V_7_00.m MATLAB ${ }^{\text {TM }}$ program file using a text editor. The program is executed in $\operatorname{MATLAB}^{\mathrm{TM}}$ by opening up MD_V_7_00.m with any text editor, changing the root directory of MD_V_7_00.m to the appropriate one on the machine, adding the directory to the MATLAB ${ }^{\mathrm{TM}}$ path, and then 
typing MD_V_7_00 in the MATLAB ${ }^{\mathrm{TM}}$ command line. The initial and final entries in the output file are shown in Appendix B.

In Appendix B, the first three bolded numbers in each italicized line are the X-,

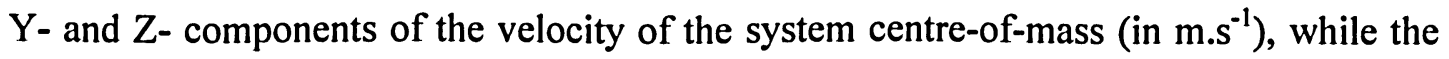
last bolded number in each italicized line is the total system energy (potential plus kinetic, in electron volts or $\mathrm{eV}$ ). Comparing corresponding values between the initial and final time steps, one can see that the momentum (or velocity of the centre-of-mass) is perfectly conserved at a value of zero to within $10^{-6}$ accuracy, while the total energy is conserved to within $10^{-2} \mathrm{eV}$, which is accepted for almost all other MD codes. Hence the author's code conserves momentum and energy very well when such conservation is expected (i.e. when PBC is not used).

As an additional observation, the last three lines of each entry is the stress tensor. Note that the shear stresses (off-diagonal terms) are zero, as expected at $0 \mathrm{~K}$ (as explained in Section 2.4.3.1). Note that the velocity components change even at $0 \mathrm{~K}$ because of the absence of any surrounding atoms to hold the atoms of the primary unit cell in place (i.e. because of the absence of PBC).

\subsection{Chapter Summary}

This chapter presented a brief overview of MD, and also presented the author's custom MD code, MD_V_7_00, which was used to do work for this thesis. Various functions used in MD_V_7_00 were described in detail, along with all the theory used during the programming stages. The code was tested by observing energy and momentum conservation on a single unit cell of copper. Although one test case is insufficient to completely validate any piece of software, the principles and code behind a general Nbody simulator are simple enough to allow for a reasonable level of confidence in using the code, given that energy and momentum were conserved very well in the test case. Furthermore, the code is used (in slightly modified forms) for all other work in this thesis, the results of which are very good. This lends more credibility to the custom MD code. 
Because the custom MD code written for this thesis is a general and powerful Nbody simulator, it would be inappropriate to attach the entire code with this thesis, lest it is misused. The flowcharts presented in Section 2.4.1 and the subsequent description of functions provide sufficient information regarding the algorithm of the code. 


\section{EMBEDDED ATOM METHOD POTENTIAL FITTING PROCEDURE FOR PURE FCC AND BCC METALS}

\subsection{Introduction to Proposed Embedded Atom Method Potential Fitting Procedure}

This part of the report presents a numerical fitting procedure for developing Embedded Atom Method (EAM) potentials for pure FCC and BCC metals which was developed by the author. The focus was on developing an approximate EAM potential that sufficed for engineering applications to pure metals. As already mentioned in Section 2.2, the EAM inter-atomic potential consists of two parts: the pair-potential and the embedding function. By assuming a parameterized form for the pair potential, the embedding function is numerically fit into the hydrostatic linear-elastic stress equations of the metal at hand. Following this, the single crystal anisotropic Young's modulus and Poisson's ratio of the metal at 293 degree Kelvin $(293 \mathrm{~K}$ ) are calculated through a uniaxial Molecular Dynamics (MD) simulation, and are compared to experimental values. The parameters of the pair potential are then changed and the embedding function recalculated until the Young's modulus and Poisson's ratio are satisfactorily predicted. Following this, a parameterized relation between temperature and kinetic energy is fit into the thermal expansion data of the metal, and a temperature dependent volume factor for calculating Young's modulus at $0 \mathrm{~K}$ and $100 \mathrm{~K}$ accurately is numerically fit. Finally, the potential is adjusted without changing the slopes (i.e. forces) to fit the cohesive energy of the metal.

The following sections present details on the proposed fitting procedure, and conclude with a test of the EAM potential obtained for copper using the proposed procedure. The thermal and elastic properties of single crystal copper are predicted using the EAM potential, and are compared to corresponding experimental values. 


\subsection{Molecular Dynamics (MD) Code for Numerical EAM Potential Fitting}

The code for fitting EAM potentials is a variant of MD_V_7_00, which has been named EAM_Potential_Fit_V_4_00. The only major difference between MD_V_7_00 and EAM_Potential_Fit_V_4_00 is the presence of code blocks related to fitting EAM potentials in EAM_Potential_Fit_V_4_00. Any additional theory for these new code blocks is presented in the following sections.

\subsection{Essential Components of the Proposed EAM Potential Fitting Procedure}

\subsubsection{Relationship between Temperature and Atomic Velocity}

It is a popular result of statistical mechanics that the average kinetic energy per axis of a large number, $N_{t o t}$, of identical particles governed by the Maxwell distribution (see Appendix E) is:

$$
\sum_{i=1}^{N} \frac{1}{2} m_{i}\left(v_{x, i}{ }^{2}\right)_{\text {avg. }}=\sum_{i=1}^{N} \frac{1}{2} m_{i}\left(v_{y, i}{ }^{2}\right)_{\text {avg. }}=\sum_{i=1}^{N} \frac{1}{2} m_{i}\left(v_{z, i}{ }^{2}\right)_{\text {avg. }}=\frac{1}{2} N_{\text {tot }} k_{B} T
$$

In equation (25), $m_{i}$ is the mass of atom $i, k_{B}$ is Boltzmann's constant and $T$ is the absolute temperature of the system. A reasonable engineering modification for metallic nuclei in a crystal lattice is taken for the proposed fitting procedure, which is to assume the following parameterized form of equation (25):

$$
\sum_{i=1}^{N} \frac{1}{2} m_{i} \bar{v}_{x, i}{ }^{2}=\sum_{i=1}^{N} \frac{1}{2} m_{i} \bar{v}_{y, i}{ }^{2}=\sum_{i=1}^{N} \frac{1}{2} m_{i} \bar{v}_{z, i}{ }^{2}=\frac{1}{2} N_{t o t} k_{B}^{\prime} T^{\mu}
$$


With this relationship, the average thermal stress on the entire crystal lattice can be calculated in equation (17). The parameter $\mu$ is adjusted during the numerical fitting procedure to fit thermal expansion data of the metal at hand as best as possible. The factor $k_{B}^{\prime}$ is taken to be numerically equivalent to $k_{B}$ (Boltzmann's constant), with units that maintain dimensional consistency of equation (26).

\subsubsection{Pair Potential for the EAM Potential: The Lennard-Jones Potential}

A modified parameterized Lennard-Jones ( $\mathrm{LJ}$ ) pair potential is assumed as the basic nuclear pair interaction for the EAM potential in this work, and is mathematically given by the following expression (the conventional LJ potential equation taken from [21] has been parameterized):

$$
U_{L V}(r)=4 \varepsilon\left[\left(\frac{\sigma}{r}\right)^{L J_{-} 1}-\left(\frac{\sigma}{r}\right)^{L J_{-} 2}\right], L J_{-} 1>L J_{-} 2
$$

, where $\varepsilon$ and $\sigma$ are the parameters defining the depth and location of the potential well respectively. The general form of the $L J$ potential for arbitrary values of $\varepsilon, \sigma, L J_{-} 1$ and $L J \_2$ is shown in Figure 2.

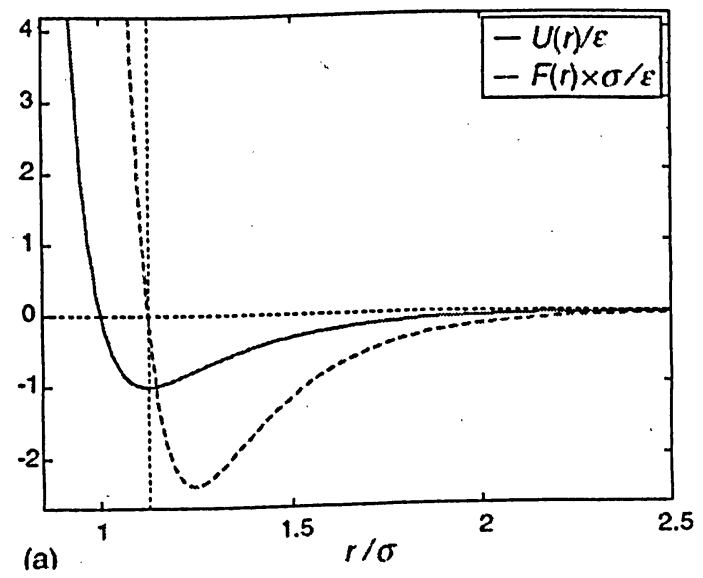

Figure 2 - General Form of the Lennard-Jones Pair Potential 
For the proposed EAM fitting procedure, $\sigma$ is chosen so that the LJ potential with $L J \_1$ and $L J \_2$ taken as 12 and 6 respectively (i.e. the conventional form of the LJ pair potential) is a minimum when the interatomic distance is equal to the nearest-neighbour distance in the perfect lattice at zero degree Kelvin (0K). If $r=r_{n}$ is the nearestneighbour distance at $0 \mathrm{~K}$, then the value of $\sigma$ for a minimum is obtained by differentiating equation (27), setting it to zero and setting $L J_{-} 1$ and $L J_{-} 2$ as 12 and 6 respectively. The resulting value of $\sigma$ is:

$$
\sigma=\left(\frac{1}{2}\right)^{1 / 6} r_{n}
$$

In equation (27), $\varepsilon, L J_{-} 1$ and $L J \_2$ are free parameters that are varied during the numerical fitting procedure to fit uniaxial elastic properties and elastic shear properties. The magnitude of force due to the $\mathrm{LJ}$ potential is calculated in the following usual way (i.e. in the way that any force is calculated from a potential):

$$
\vec{F}_{L J}=-\vec{\nabla} U_{L J}(r)
$$

, where $\vec{\nabla}$ is the gradient of a scalar field.

\subsubsection{Atomic Electron Density Calculation using WinGAMESS}

In general, as seen from equation (3), the embedding function $\Psi$ at the location of a nucleus is a function of total electron density at the position of that nucleus in the crystal lattice, excluding the self-contribution of that nucleus. While using the EAM potential, the total electron density at a point is approximated as the sum of individual atomic electron densities at that point from surrounding atoms, which can be inferred from equation (4). The atomic electron density of the metal is obtained from a numerical 
quantum mechanical Hartree-Fock Self-Consistent-Field (HF SCF) computation on one atom of the metal, using the numerical quantum mechanical freeware package WinGAMESS ${ }^{* *}$. Details on the HF SCF method, and the WinGAMESS input file for copper, are given in Appendix D.

\subsubsection{Varying the Total Electron Density during the EAM Fitting Process}

The essential goal of the numerical EAM fitting procedure is to determine the embedding function, $\Psi$, as a function of total electron density. From equation (4), it is evident that the only method of changing the total electron density at a point while using the EAM potential is to change inter-nuclear distances in the crystal lattice. Calculating the embedding function then requires known relationships between the crystal lattice at different inter-nuclear distances, and the force from the EAM potential (equation (5)).

From a stress engineering point of view, stress relationships are the most convenient since a method of calculating stress is already known in the form of the virial stress tensor. It is a tried and tested fact in stress engineering that the Von-Mises yield criterion is the most satisfactory yield criterion for ductile metals (both single crystals and poly-crystalline metals), which has made it the principal predictor of yielding in ductile metals in the industry. The Von-Mises yield criterion states that yielding of a ductile metal does not take place until the Von-Mises stress (an equivalent stress based on distortion strain energy) reaches the uniaxial yield stress from tensile tests. Since the distortion strain energy associated with a state of hydrostatic stress is always zero, a ductile metal does not yield under hydrostatic loading or straining under the Von-Mises yield criterion. Hence large hydrostatic tensile and compressive strains $( \pm 30 \%$ engineering strain is used for this thesis) can be applied to the metal crystal lattice, and the resulting stresses calculated using the virial stress tensor must still satisfy the following elastic relationship (which can easily be derived from general elastic stressstrain relationships for cubic crystals):

\footnotetext{
"* GAMESS Home Page: http://www.msg.ameslab.gov/GAMESS/GAMESS.html
} 


$$
\frac{\sigma_{x x}}{\varepsilon_{x x}}=\frac{\sigma_{y y}}{\varepsilon_{y y}}=\frac{\sigma_{z z}}{\varepsilon_{z z}}=\frac{E}{1-2 v}
$$

$E$ is the single crystal Young's modulus of the metal and $v$ is the single crystal Poisson's ratio of the metal. For a single crystal, $E$ and $v$ exhibit anisotropy along different crystallographic directions. Using the three cubic elastic constants $c_{11}, c_{12}$ and $c_{44}$ for the metal (which are always specified in the coordinate system that uses the standard, three mutually perpendicular crystallographic axes [100], [010] and [001]; this system is also the coordinate system used by the custom MD code), the (anisotropic) $E, v$ and $G$ (shear modulus) values in this standard coordinate system are calculated using the following equations [27]:

$$
\begin{gathered}
E=\frac{c_{11}^{2}+c_{11} c_{12}-2 c_{12}{ }^{2}}{c_{11}+c_{12}} \\
v=\frac{c_{12}}{c_{11}+c_{12}} \\
G=c_{44}
\end{gathered}
$$

Conversely:

$$
\begin{gathered}
c_{11}=E\left(\frac{1-v}{1-v-2 v^{2}}\right) \\
c_{12}=E\left(\frac{v}{1-v-2 v^{2}}\right) \\
c_{44}=G
\end{gathered}
$$


Equation (30) is a vital relationship in the proposed EAM fitting procedure. The importance of equation (30) is that it allows the total electron density to be varied significantly, and yet maintains a definite known relationship among all quantities of interest. Hence the slope of the embedding function can be calculated properly for a wide range of total electron densities. The slope is then numerically integrated using the trapezoid rule to obtain the embedding function.

\subsubsection{Fitting the Cohesive Energy of the Metal}

The cohesive energy of the metal is the energy required to completely remove an atom of the metal from the crystal lattice. Since the externally applied mechanical stress tensor is dependent only upon inter-atomic forces (equation (17)), which are in turn dependent only upon the gradient of the EAM potential (equation (5)), the fitting procedure is unaltered if a constant is added to the entire potential. This constant is calculated such that the cohesive energy of the metal at $293 \mathrm{~K}$ predicted by the EAM potential matches experimental values.

\subsection{Proposed Numerical EAM Potential Fitting Procedure}

Figure 72 and Figure 73 in Appendix $C$ show a flowchart for the proposed numerical EAM potential fitting procedure. The flowchart is also the algorithm used for the custom MD code that performs the numerical fitting. A temperature dependent volume factor is used in the virial stress equation (equation (17)) to scale the volume by an amount required to properly predict Young's modulus at $0 \mathrm{~K}$. A power law is fit through this calculated factor, a factor of 1 at $293 \mathrm{~K}$ (since all data at $293 \mathrm{~K}$ require no factor by design of the fitting procedure), and the experimental value of Young's modulus at $100 \mathrm{~K}$. 


\subsection{Results: EAM Potential for Copper}

Using the proposed numerical fitting procedure (Figure 72 and Figure 73), the EAM potential for copper was determined. The electron density output from WinGAMESS was found to be well approximated by the following power fit in $R$ (distance from nuclear centre):

$$
\rho=0.268619 \cdot R^{-5.239274}
$$

In equation (37), $R$ is in Angstroms and is in the range 0.4 Angs $\leq R \leq 6$ Angs. No atom in a metal is expected to come close to another by less than 0.4 Angstroms (which is very conservative itself), while the electron density beyond 6 Angstroms is negligible for copper (from the WinGAMESS output). In using equation (37), the resulting unit of $\rho$ is Coulomb.Angstrom ${ }^{-3}\left(C . \AA^{-3}\right)$. A plot of equation (37) is shown in Figure 3.

The modified Lennard-Jones pair potential was iteratively found to be the following function of inter-nuclear distance $R$ (where $R$ is in Angstroms):

$$
\phi=225.344\left[\left(\frac{2.269792}{R}\right)^{6.01}-\left(\frac{2.269792}{R}\right)^{6}\right]
$$

In equation (38), $\phi$ is in $\mathrm{eV}(1 \mathrm{eV}=1.602 \mathrm{e}-19$ Joules or $\mathrm{J})$. A plot of equation (38) is shown in Figure 4.

The iterated, integrated and cohesion-energy-shifted embedding function values are plotted in Figure 5 as a function of total electron density. Because the values are numerically determined, there is no need to fit a function to the data: it can be read from a text file and used as is (interpolating for intermediate data points whenever required).

The temperature dependent volume factor was found to be: 


$$
V_{\text {_factor }}=1.288455 \mathrm{e}-009\left(\frac{\mathrm{T}}{293}\right)^{3}+0.967591
$$

The volume $V$ in equation (17) is multiplied by this factor at the simulation temperature before calculating the virial stress.

Finally, the temperature exponent $\mu$ for average kinetic energy was calculated as 1.221. Hence:

Avg. K.E. per axis $=\sum_{i=1}^{N} \frac{1}{2} m_{i} \bar{v}_{x, l}{ }^{2}=\sum_{i=1}^{N} \frac{1}{2} m_{i} \bar{v}_{y, i}{ }^{2}=\sum_{i=1}^{N} \frac{1}{2} m_{l} \bar{v}_{z, i}{ }^{2}=\frac{1}{2} N_{t o t} k_{B}^{\prime} T^{1.221}$

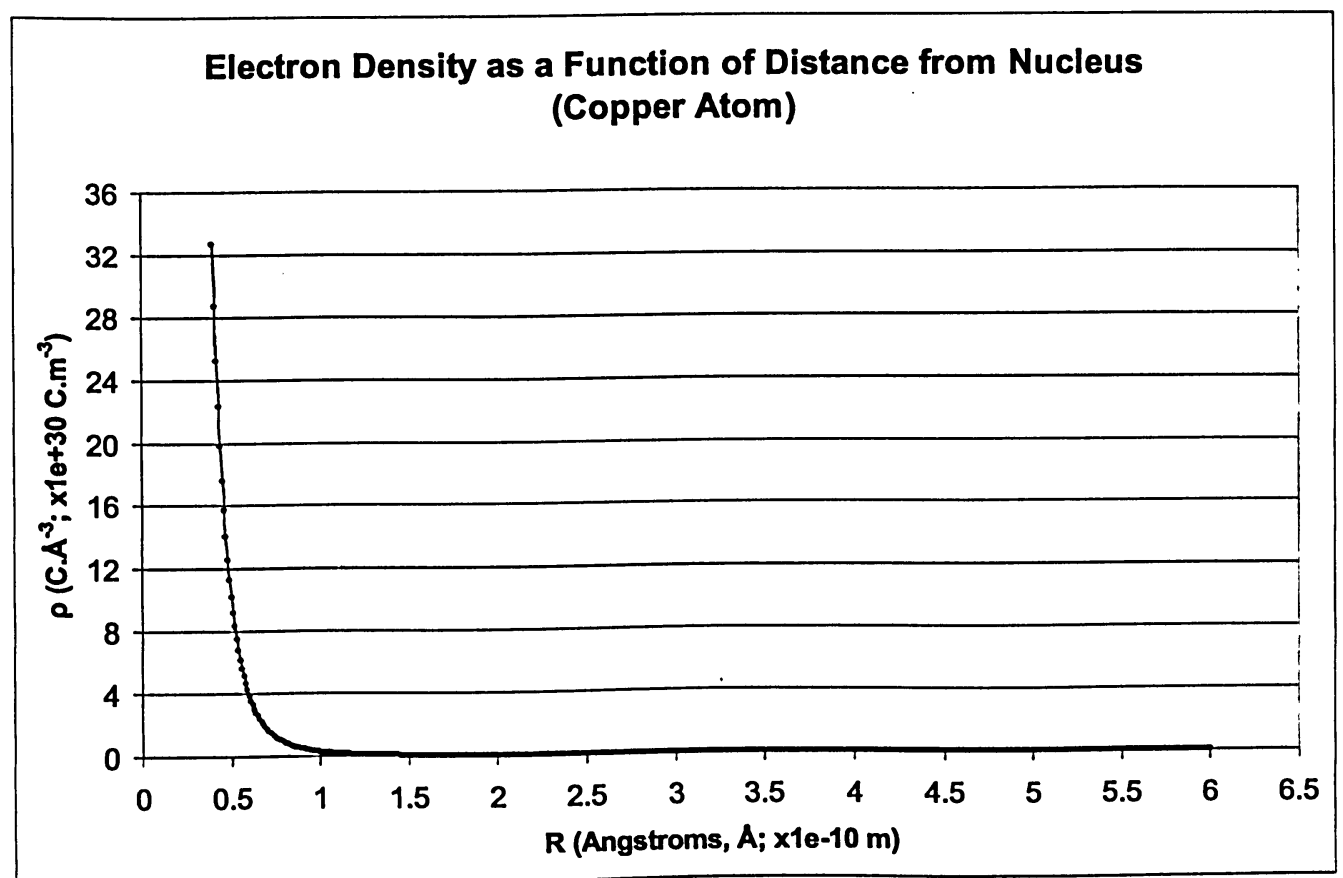

Figure 3 - Electron Density of Copper Atom (WinGAMESS Output) 
EMBEDDED ATOM METHOD POTENTIAL FITTING PROCEDURE FOR PURE FCC AND BCC METALS

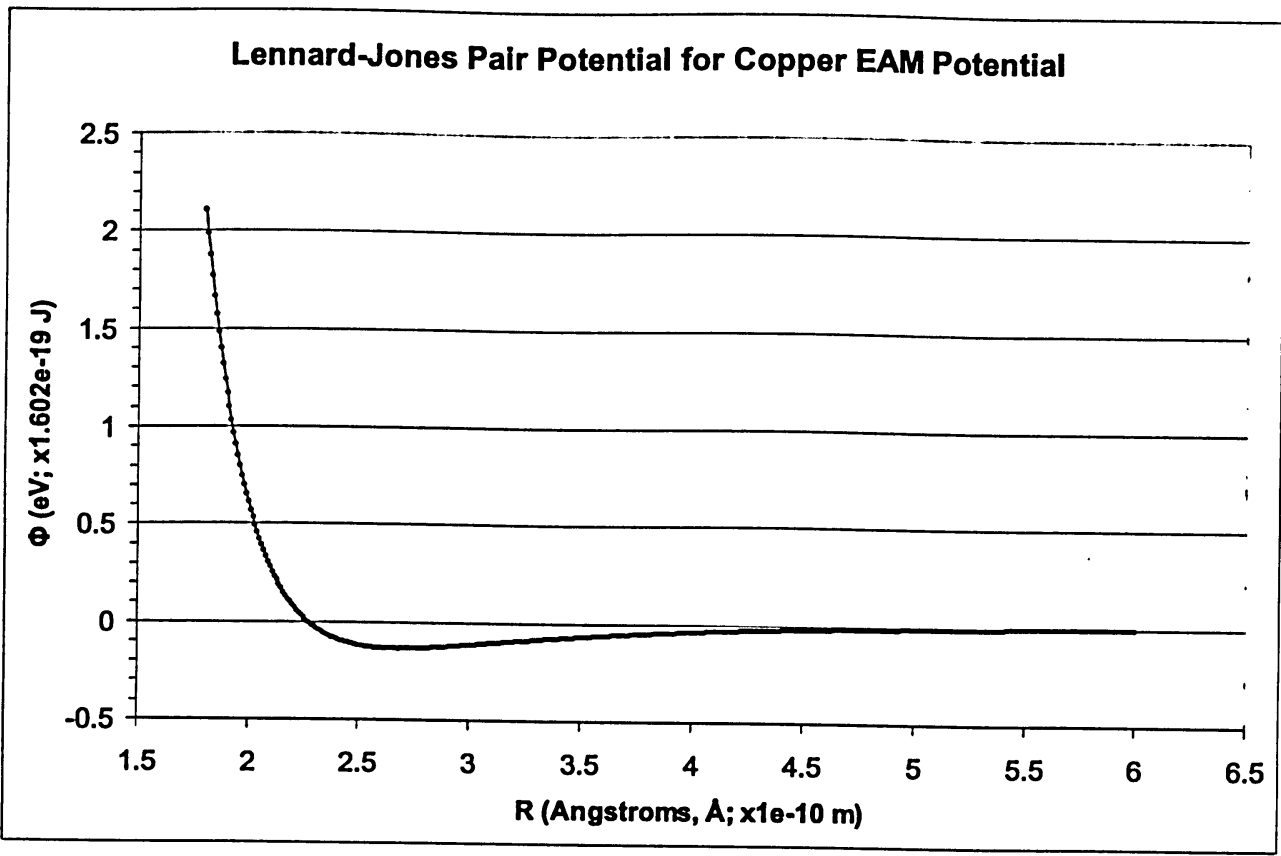

Figure 4 - Lennard-Jones Pair Potential for Copper EAM Potential

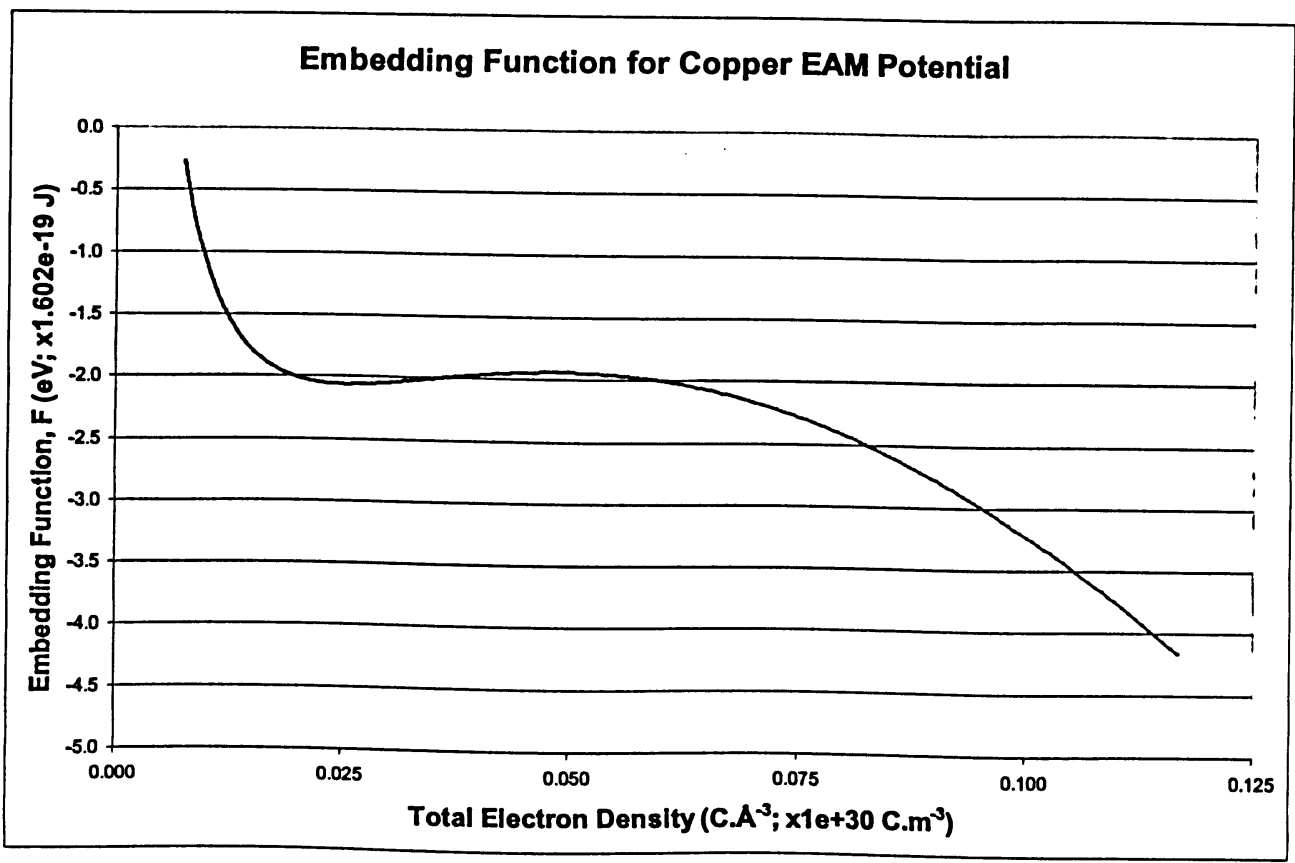

Figure 5 - Embedding Function for Copper EAM Potential 
In Figure 5, the embedding function for low electron densities appears to be becoming positive. However, intuitively, the embedding function must be zero and must have zero slope at zero total electron density. To overcome this, a smooth function can be filled in between zero total electron density and the first electron density in Figure 5. This would enable the prediction of failure for perfect crystals (identified when atoms in the MD simulation are no longer stably bound to one-another). However, for reasons to be given in Section 3.7, this effort is unwarranted at this stage. Moreover, it does not present a problem in this thesis since such high unrealistic tensile strains were not applied in the test cases.

\subsection{EAM Potential Testing: Thermal and Elastic Properties of Single Crystal Copper}

\subsubsection{Test Case 1: Lattice Constants of Copper Single Crystal at Various Temperatures}

The first test case uses the EAM potential and temperature parameter, $\mu$, with the custom MD code MD_V_7_00 to determine the equilibrium lattice constant of copper at various temperatures ranging from $0 \mathrm{~K}$ to $1300 \mathrm{~K}$. The results for various temperatures, and the corresponding experimental values, are given in Table 1.

As can be seen from Table 1, the agreement between results using the numerically fitted EAM potential and experimental results are very good, except very near $0 \mathrm{~K}$. For all practical applications, the temperature lies between $50 \mathrm{~K}$ and $1000 \mathrm{~K}$, for which the agreement is excellent (showing a maximum deviation of $0.0430 \%$ from experimental results). The agreement is exact at room temperature, which was enforced in the numerical procedure. 


\section{BCC METALS}

Table 1 - Calculated Equilibrium Lattice Constants of Copper at Various Temperatures

\begin{tabular}{|l|c|c|c|}
\hline \multirow{2}{*}{$\begin{array}{c}\text { Absolute Temperature } \\
(\mathbf{K})\end{array}$} & \multicolumn{2}{|c|}{ Lattice Constant (Angstroms) } & \multirow{2}{*}{ \% Difference } \\
\cline { 2 - 3 } & Calculated with Custom MD Code & Experimental [28] & 0.0817 \\
\hline 0 & 3.600343 & 3.603287 & 0.0616 \\
\hline 25 & 3.601069 & 3.603287 & 0.0408 \\
\hline 50 & 3.602035 & 3.603504 & 0.0144 \\
\hline 100 & 3.604288 & 3.604806 & 0.0031 \\
\hline 200 & 3.609538 & 3.609650 & 0.0000 \\
\hline 293 (room temp.) & 3.615000 & 3.615000 & 0.0055 \\
\hline 400 & 3.621778 & 3.621579 & 0.0112 \\
\hline 500 & 3.628491 & 3.628086 & 0.0182 \\
\hline 600 & 3.635509 & 3.634846 & 0.0276 \\
\hline 700 & 3.642792 & 3.641787 & 0.0374 \\
\hline 800 & 3.650309 & 3.648945 & 0.0430 \\
\hline 900 & 3.658037 & 3.656464 & 0.0430 \\
\hline 1000 & 3.665958 & 3.664381 & 0.0237 \\
\hline 1200 & 3.682318 & 3.681444 & 0.0000 \\
\hline 1300 & 3.690734 & 3.690734 & \\
\hline
\end{tabular}

\subsubsection{Test Case 2: Uniaxial Properties of Copper Single Crystal at 293K}

The second test case uses the EAM potential and temperature parameter, $\mu$, with MD_V_7_00 to determine the (anisotropic) Young's modulus and Poisson's ratio of single crystal copper at $293 \mathrm{~K}$ through uniaxial tensile and compressive stresses on the crystal lattice.

For the tensile stress, MD_V_7_00 was used to calculate strains needed to obtain a mechanical virial stress tensor of:

$$
\underline{\sigma}=\left[\begin{array}{ccc}
100 & 0 & 0 \\
0 & 0 & 0 \\
0 & 0 & 0
\end{array}\right] \mathrm{MPa}
$$

MD_V_7_00 determined the required engineering strain tensor (using the procedure outlined in Section 2.4.3.3) to be: 


$$
\underline{\varepsilon}=\left[\begin{array}{ccc}
0.149026 & 0 & 0 \\
0 & -0.061871 & 0 \\
0 & 0 & -0.061871
\end{array}\right] \% \text { strain }
$$

Young's modulus is then:

$$
E=\frac{\sigma_{x x}}{\varepsilon_{x x}}=\frac{100 e+06}{0.149026 e-02}=67.102326 e+09 \mathrm{~Pa}=67.1023 \mathrm{GPa}
$$

Poisson's ratio is:

$$
v=-\frac{\varepsilon_{y y}}{\varepsilon_{x x}}=-\frac{\varepsilon_{z z}}{\varepsilon_{x x}}=-\frac{-0.061871}{0.149026}=0.415167
$$

For the compressive stress, MD_V_7_00 was used to calculate strains needed to obtain a mechanical virial stress tensor of:

$$
\underline{\sigma}=\left[\begin{array}{ccc}
-100 & 0 & 0 \\
0 & 0 & 0 \\
0 & 0 & 0
\end{array}\right] \mathrm{MPa}
$$

MD_V_7_00 determined the required engineering strain tensor to be:

$$
\underline{\varepsilon}=\left[\begin{array}{ccc}
-0.150956 & 0 & 0 \\
0 & 0.063144 & 0 \\
0 & 0 & 0.063144
\end{array}\right] \% \text { strain }
$$

Young's modulus is then: 


$$
E=\frac{\sigma_{x x}}{\varepsilon_{x x}}=\frac{-100 e+06}{-0.150956 e-02}=6.6244306 e+09 \mathrm{~Pa}=66.2443 \mathrm{GPa}
$$

Poisson's ratio is:

$$
v=-\frac{\varepsilon_{y y}}{\varepsilon_{x x}}=-\frac{\varepsilon_{z z}}{\varepsilon_{x x}}=-\frac{0.063144}{-0.150956}=0.418294
$$

The elastic constants $c_{11}, c_{12}$ and $c_{44}$ of pure copper in the standard [100]-[010]-[001] crystal coordinate system are $164.84 \mathrm{GPa}, 117.74 \mathrm{GPa}$ and $75.4 \mathrm{GPa}$ respectively [29]. Using equations (31) and (32), the anisotropic $E$ and $v$ values in the standard crystal coordinate system are calculated as $66.7341 \mathrm{GPa}$ and 0.416649 respectively. The agreement is once again excellent, with less than $1 \%$ differences between corresponding values.

As the stress values are increased, the error in the results increases, but not significantly (less than $2 \%$ difference at $300 \mathrm{MPa}$ stress). This is acceptable from an engineering stand-point.

\subsubsection{Test Case 3: Hydrostatic Elasticity of Single Crystal Copper at 293K}

The third test case uses the EAM potential and temperature parameter, $\mu$, with MD_V_7_00 to determine the (anisotropic) hydrostatic elasticity of single crystal copper at $293 \mathrm{~K}$ through hydrostatic tensile and compressive stresses on the crystal lattice.

For the tensile stress, MD_V_7_00 was used to calculate strains needed to obtain a mechanical virial stress tensor of:

$$
\underline{\sigma}=\left[\begin{array}{ccc}
100 & 0 & 0 \\
0 & 100 & 0 \\
0 & 0 & 100
\end{array}\right] \mathrm{MPa}
$$


MD_V_7_00 determined the required engineering strain tensor to be:

$$
\underline{\varepsilon}=\left[\begin{array}{ccc}
0.024980 & 0 & 0 \\
0 & 0.024980 & 0 \\
0 & 0 & 0.024980
\end{array}\right] \% \text { strain }
$$

From equation (30), hydrostatic elasticity is then:

$$
\frac{E}{1-2 v}=\frac{\sigma_{x x}}{\varepsilon_{x x}}=\frac{100 e+06}{0.024980 e-02}=400.320577 e+09 \mathrm{~Pa}=400.3206 \mathrm{GPa}
$$

For the compressive stress, MD_V_7_00 was used to calculate strains needed to obtain a virial stress tensor of:

$$
\underline{\sigma}=\left[\begin{array}{ccc}
-100 & 0 & 0 \\
0 & -100 & 0 \\
0 & 0 & -100
\end{array}\right] \mathrm{MPa}
$$

MD_V_7_00 determined the required engineering strain tensor to be:

$$
\underline{\varepsilon}=\left[\begin{array}{ccc}
-0.024980 & 0 & 0 \\
0 & -0.024980 & 0 \\
0 & 0 & -0.024980
\end{array}\right] \% \text { strain }
$$

From equation (30), hydrostatic elasticity is then:

$$
\frac{E}{1-2 v}=\frac{\sigma_{x x}}{\varepsilon_{x x}}=\frac{-100 e+06}{-0.024980 e-02}=400.320577 e+09 \mathrm{~Pa}=400.3206 \mathrm{GPa}
$$

The experimental value of single crystal hydrostatic elasticity for copper is: 


$$
\frac{E}{1-2 v}=\frac{66.7341 e+09}{1-2(0.416649)}=400.319732 e+09 \mathrm{~Pa}=400.3197 \mathrm{GPa}
$$

, where $E$ and $v$ were calculated from the experimental single crystal elastic constants using equations (31) and (32), as done in section 3.6.2. The agreement is once again excellent (negligible \% difference).

\subsubsection{Test Case 4: Shear Modulus of Copper at 293K}

The fourth test case uses the EAM potential and temperature parameter, $\mu$, with MD_V_7_00 to determine the (anisotropic) shear modulus of single crystal copper at 293K through hydrostatic tensile and compressive stresses on the crystal lattice.

MD_V_7_00 applies a shear strain, $\varepsilon_{x y}$ for example, in the following conventional way:

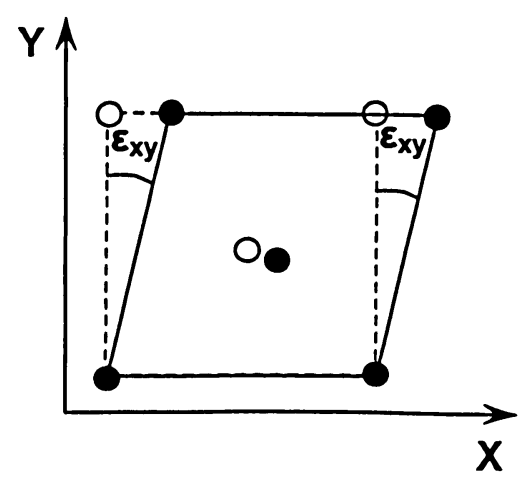

-.... Undeformed crystal and atoms

Deformed crystal and atoms

Figure 6 - Convention for Shear Strain Application in Custom MD Code

For positive shear strain, MD_V_7_00 was used to calculate stresses resulting from an engineering strain tensor of: 


$$
\underline{\varepsilon}=\left[\begin{array}{ccc}
0 & 0.1 & 0 \\
0.1 & 0 & 0 \\
0 & 0 & 0
\end{array}\right] \% \text { strain }
$$

MD_V_7_00 determined the resulting stress tensor to be:

$$
\underline{\sigma}=\left[\begin{array}{ccc}
-0.277317 & 75.771688 & 0 \\
75.771688 & -0.149306 & 0 \\
0 & 0 & 0.032640
\end{array}\right] \mathrm{MPa}
$$

The small normal stresses are due to the sensitivity of the virial stress tensor (equation (17)) to atomic positions. The magnitudes are not cause for concern from an engineering stand-point. The (anisotropic) single crystal shear modulus is then:

$$
G=\frac{\sigma_{x y}}{\varepsilon_{x y}}=\frac{75.771688 \mathrm{e}+06}{0.1 e-02}=75.771688 e+09 \mathrm{~Pa}=75.7717 \mathrm{GPa}
$$

For negative shear strain, $M D_{-} V_{-}$__ 00 was used to calculate stresses resulting from an engineering strain tensor of:

$$
\underline{\varepsilon}=\left[\begin{array}{ccc}
0 & -0.1 & 0 \\
-0.1 & 0 & 0 \\
0 & 0 & 0
\end{array}\right] \% \text { strain }
$$

MD_V_7_00 determined the resulting stress tensor to be:

$$
\underline{\sigma}=\left[\begin{array}{ccc}
-0.277317 & -75.771688 & 0 \\
-75.771688 & -0.149306 & 0 \\
0 & 0 & 0.032640
\end{array}\right] \mathrm{MPa}
$$


The (anisotropic) single crystal shear modulus is then:

$$
G=\frac{\sigma_{x y}}{\varepsilon_{x y}}=\frac{-75.771688 \mathrm{e}+06}{-0.1 e-02}=75.771688 e+09 \mathrm{~Pa}=75.7717 \mathrm{GPa}
$$

The experimental value of single crystal shear modulus for copper is obtained using equation (33) and the value of the elastic constant $c_{44}$ for copper (from Section 3.6.2):

$$
G=c_{44}=75.4 \mathrm{GPa}
$$

The results show a deviation of less than $0.5 \%$ from the expected result, which is once again excellent.

\subsubsection{Test Case 5: Isothermal Young's Modulus and Poisson's Ratio of Copper from 0K to $300 \mathrm{~K}$}

The fifth and final test case uses the EAM potential and the thermal virial stresses with MD_V_7_00 to determine the (anisotropic) isothermal Young's modulus and Poisson's ratio of single crystal copper at various temperatures from $0 \mathrm{~K}$ to $300 \mathrm{~K}$ through a uniaxial tensile stress on the crystal lattice. The MD results are compared to expected values, calculated from isothermal elastic constants data $[29,30]$ using equations (31) and (32). At each temperature, MD_V_7_00 was used to calculate strains needed to obtain a mechanical virial stress tensor of:

$$
\underline{\sigma}=\left[\begin{array}{ccc}
100 & 0 & 0 \\
0 & 0 & 0 \\
0 & 0 & 0
\end{array}\right] \mathrm{MPa}
$$

The results are shown in Table 2. 
Table 2 - Isothermal Young's Modulus and Poisson's Ratio of Copper at Various Temperatures for 100 MPa Uniaxial Stress (calculated using custom MD code)

\begin{tabular}{|c|c|c|c|c|}
\hline \multirow{2}{*}{$\begin{array}{c}\text { Absolute } \\
\text { Temperature (K) }\end{array}$} & \multicolumn{2}{|c|}{ Normal Strains } & \multirow{2}{*}{$\begin{array}{c}\text { Young's Modulus } \\
\text { (Gpa; x1e+9 Pa) }\end{array}$} & \multirow{2}{*}{ Poisson's Ratio } \\
\hline & XX Strain (\%) & YY Strain $=$ ZZ Strain (\%) & & \\
\hline$\overline{0}$ & 0.13787931 & -0.05669823 & 72.5272 & 0.411216 \\
\hline 25 & 0.13807888 & -0.05696229 & 72.4224 & 0.412534 \\
\hline 50 & 0.13840861 & -0.05727461 & 72.2498 & 0.413808 \\
\hline 100 & 0.13960898 & -0.05774278 & 71.6286 & 0.413604 \\
\hline 200 & 0.14323699 & -0.05937951 & 69.8144 & 0.414554 \\
\hline 293 (room temp.) & 0.14902613 & -0.06187068 & 67.1023 & 0.415167 \\
\hline
\end{tabular}

Comparison with expected values, calculated from isothermal elastic constants data [30] using equations (31) and (32), are presented in Figure 7 and Figure 8. As seen from Figure 7, the variation of Young's modulus with temperature is captured quite well, with a difference of less than $0.5 \mathrm{GPa}$ in all cases. This is within acceptable error. The variation of Poisson's ratio with temperature is also predicted fairly accurately (as seen from Figure 8). In particular, the Poisson's ratios between 50K and $293 \mathrm{~K}$ are close to the expected value. Judging by the trend of the data in Figure 8, this agreement is expected to continue far above $293 \mathrm{~K}$. Hence the potential can be used with confidence in a wide temperature range.

\subsubsection{Summary of Results for Anisotropic Mechanical Properties of Single Crystal Copper at 293K}

Table 3 summarizes the anisotropic mechanical properties of single crystal copper at 293K obtained from MD simulations (Sections 3.6.2 through 3.6.4), and compares then to reference mechanical properties taken from the literature. References for the literature values have already been given in the preceding sections. 


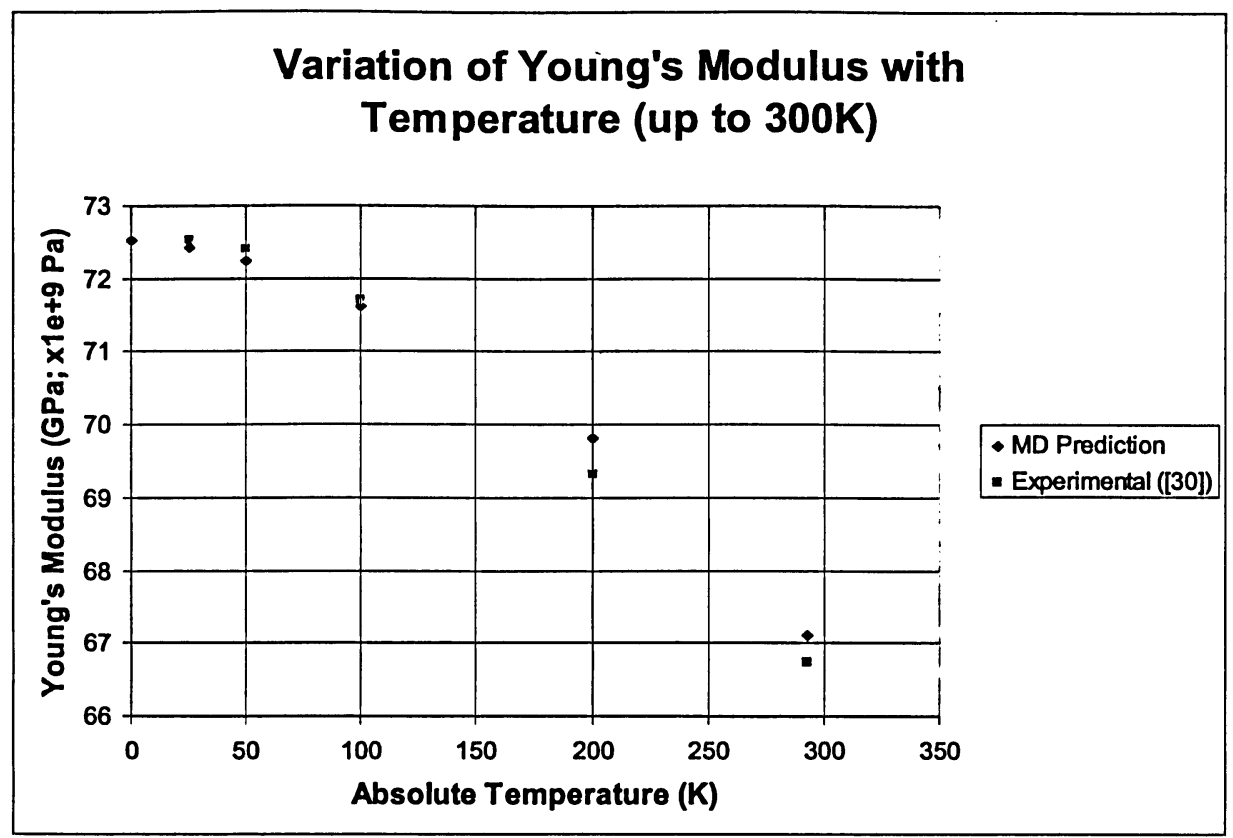

Figure 7 - Variation of Young's Modulus with Temperature (up to 300K)

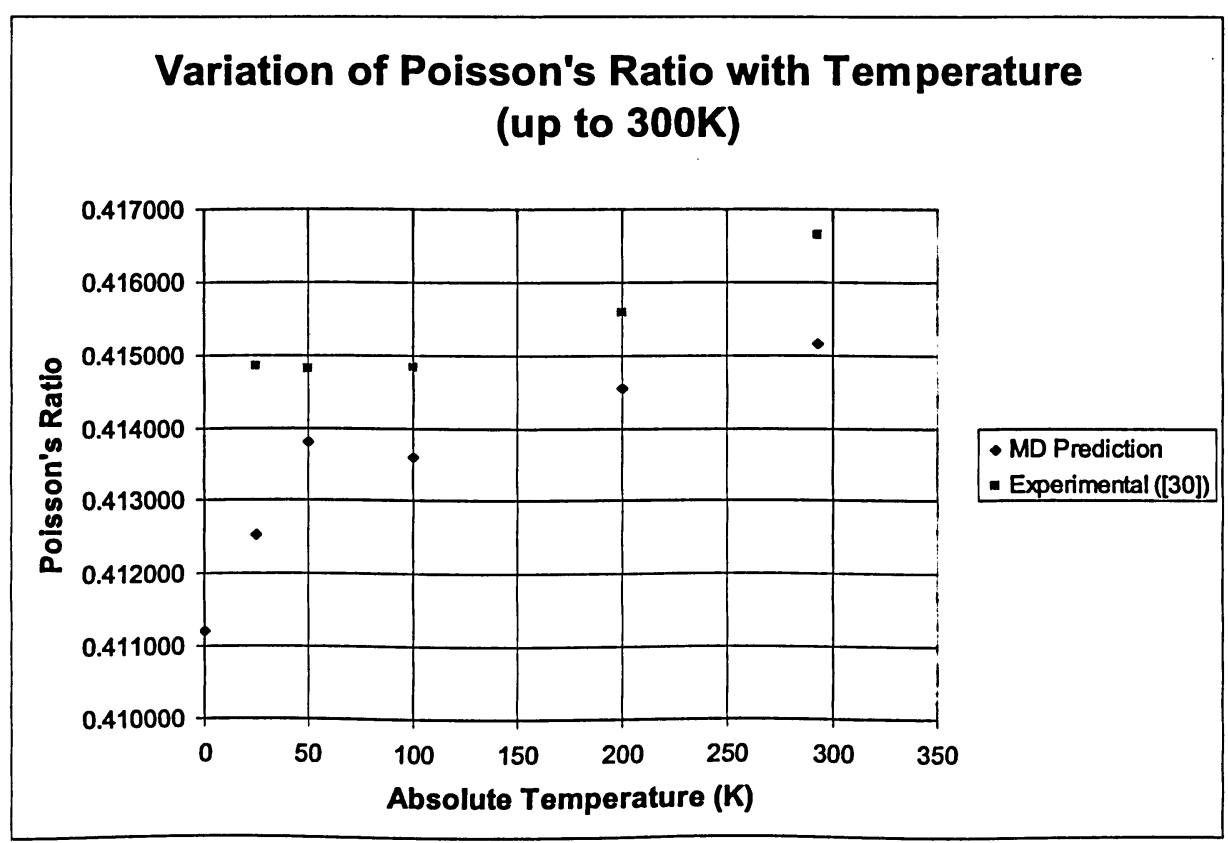

Figure 8 - Variation of Poisson's Ratio with Temperature (up to 300K) 
Table 3 - Anisotropic Mechanical Properties of Single Crystal Copper at 293K

\begin{tabular}{|l|c|c|c|}
\hline \multicolumn{1}{|c|}{ Mechanical Property } & MD Simulation Result & Reference Value & $\begin{array}{c}\text { Percentage } \\
\text { Deviation }\end{array}$ \\
\hline $\begin{array}{l}\text { Tensile Young's modulus for loading along } \\
\text { [100] axis at 293K (GPa; } x \text { le+9 Pa) }\end{array}$ & 67.1023 & 66.7341 & $0.5517 \%$ \\
\hline $\begin{array}{l}\text { Tensile Poisson's ratio for loading along } \\
{[100] \text { axis at 293K }}\end{array}$ & 0.415167 & 0.416649 & $0.3557 \%$ \\
\hline $\begin{array}{l}\text { Compressive Young's modulus for loading } \\
\text { along [100] axis at 293K (GPa; } x \text { le+9 Pa) }\end{array}$ & 66.2443 & 66.7341 & $0.7340 \%$ \\
\hline $\begin{array}{l}\text { Compressive Poisson's ratio for loading } \\
\text { along [100] axis at 293K }\end{array}$ & 0.418294 & 0.416649 & $0.3948 \%$ \\
\hline $\begin{array}{l}\text { Tensile hydrostatic elastic modulus for } \\
\text { loading along [100]-[010]-[001] axes at } \\
\text { 293K (GPa; } \text { xle+9 Pa) }\end{array}$ & 400.3206 & 400.3197 & $0.0002 \%$ \\
\hline $\begin{array}{l}\text { Compressive hydrostatic elastic modulus } \\
\text { for loading along [100]-[010]-[001] axes at } \\
\text { 293K (GPa; } x \text { le+9 Pa) }\end{array}$ & 400.3206 & 400.3197 & $0.0002 \%$ \\
\hline $\begin{array}{l}\text { Positive shear modulus for shearing about } \\
\text { [001] axis at 293K (GPa; } x \text { le+9 Pa) }\end{array}$ & 75.7717 & 75.4 & $0.4930 \%$ \\
\hline $\begin{array}{l}\text { Negative shear modulus for shearing about } \\
{[001] \text { axis at 293K (GPa; } x \text { le+9 Pa) }}\end{array}$ & 75.7717 & 75.4 & $0.4930 \%$ \\
\hline
\end{tabular}

\subsection{Discussion}

The numerically fit EAM potential for copper is found to predict thermal and elastic properties of copper single crystal satisfactorily. The deviation of the lattice constant at various temperatures from experimental values is less than $0.1 \%$ as seen from Table 1 , while the single crystal isothermal anisotropic elastic properties are predicted within $1 \%$ of experimental values. Hence as per these data, the proposed numerical fitting procedure seems to produce an excellent EAM potential for engineering applications over a wide temperature range.

The numerical fitting procedure has only been demonstrated with pure copper (an FCC metal) in this thesis. However, none of the concepts or principles used is specific to FCC or BCC crystals alone. The fitting procedure is very general and can therefore be implemented for any FCC or BCC metal.

It might be noted that only elastic properties have been considered for verification purposes in this thesis. Because of the use of the Von-Mises yield criterion in the fitting 
algorithm, plasticity has no bearing on the resulting EAM potential except possibly at extremely large and unrealistic tensile hydrostatic strains, as already mentioned. Moreover, prediction of plastic properties using MD simulation of perfect crystals is difficult and erroneous: it is well known that perfect crystals have extremely high theoretical yield stresses which can never be realized in experiments due to the presence of crystal defects that reduce the strength by several orders of magnitude. The only way to realistically capture plastic behaviour then is to simulate crystals with defects using $\mathrm{MD}$, which is a challenge in itself and has not been attempted in this thesis. No effort has been made to fit the theoretical yield strength of the perfect crystal since this value is extremely high and therefore unrealistic. Ultimately, EAM potentials are used to model crystal defects in single crystals or grains in polycrystalline material, which allow for proper plastic flow mechanisms.

Finally, it is emphasized that the proposed fitting procedure is not applicable to alloys, but only to pure metals. A similar procedure can be developed for alloys, but is not attempted in this thesis.

\subsection{Chapter Summary}

An engineering-oriented numerical fitting procedure for developing Embedded-AtomMethod (EAM) potentials for pure FCC and BCC metals was developed in this chapter. The fitting method fits the embedding function of the EAM potential to thermal and single crystal elastic properties of the metal. A systematic procedure for varying the EAM potential ultimately ensures that all these properties are predicted as accurately as possible. The numerical procedure was used to develop the EAM potential for copper single crystal, which was subsequently tested in a variety of test cases and gave good results. In conclusion, the proposed EAM fitting procedure promises to be a relatively easy and practical method for obtaining accurate EAM potentials for FCC and BCC metal single crystals. 


\section{APPLICATION OF MOLECULAR DYNAMICS WITH THE EMBEDDED ATOM METHOD POTENTIAL}

\subsection{Overview of Applications}

This chapter presents a study of virial stress increment patterns produced in a perfect copper crystal lattice with voids of definite geometries. The stress patterns are obtained from MD simulations of the copper crystal lattice using MD_V_7_00. The EAM potential developed for copper in Section 3.5 is used as the inter-atomic potential. Three voids are investigated in this study: an infinite, perfectly cylindrical void in the interior of the lattice; an infinite, semi-cylindrical notch at a free surface of the lattice ; and an infinite, elliptical void in the interior of the lattice. The lattices and voids are constructed and dynamically relaxed through MD simulation. It should be noted that only elastic strains are applied for all cases. Contour plots for all three voids, for a uniaxial far-field stress of $100 \mathrm{MPa}$, are presented. A qualitative comparison with existing data is not possible in this case, since studies on atomic-level voids of these geometries are extremely rare. Hence this chapter simply presents simulated results, without providing any comparison.

Isothermal conditions (at room temperature of $293 \mathrm{~K}$ ) are imposed during the MD simulation. The object is to study virial stress increments, and not the absolute virial stresses, since the absolute stress on one atom is meaningless in the context of failure prediction: an atom will never break apart or fail under the applied loads. Realistically, crystal behaviour depends on the increment of atomic stresses from the base (undeformed) state, whatever state that might be. Hence all stress patterns shown in this chapter are records of stress increments (and not absolute stresses) from the base state. In this case, the base state is simply the perfect copper crystal lattice with the appropriate lattice constant at $293 \mathrm{~K}$ and the void, relaxed using MD under a zero engineering strain tensor. The deformed state is simply the perfect copper crystal lattice 
with the appropriate lattice constant at $293 \mathrm{~K}$ and the void, relaxed using MD under the desired non-zero engineering strain tensor. The following sections present all three applications, along with the resulting stress increment contour plots.

\subsection{Application 1: Elastically Stressed Infinite, Perfectly Cylindrical Void in a Perfect Copper Crystal Lattice}

\subsubsection{Application Objective}

This application uses the copper EAM potential and MD_V_7_00 to perform a MD simulation of an infinite, perfectly cylindrical void in an infinite copper crystal lattice. The copper lattice with the void in it is first generated in MD_V_7_00, and a strain tensor corresponding to a far-field (bulk) uniaxial stress of $100 \mathrm{MPa}$ in the $\mathrm{XX}$ direction is then applied to the whole lattice. The atoms are then allowed to relax through a damped MD simulation. Appropriate damping (defined according to convention as the product of a damping constant and the atomic velocities) ensures that the atomic velocities die down to zero fairly quickly. The virial stress on each atom is then calculated and recorded. A strain-free version is then simulated to calculate the base (undeformed) local stresses at zero far-field base stress. The uniaxial results are then subtracted from the reference results to yield the net stress increment of each atom. These stress increments are plotted as contour plots for all components of stress. Periodic Boundary Condition (PBC) is used to simulate the infinite surrounding lattice (already described in Section 2.4.3.4).

\subsubsection{Infinite Copper Lattice Specifications}

A $15 \times 15 \times 1$ (in the order XYZ in the standard [100]-[010]-[001] crystal coordinate system) unit cell nano-layer of copper (each with a lattice constant of 3.615 Angstroms, i.e. each at 293K) is first generated using MD_V_7_00. A 7 Angstroms radius cylindrical 
void with its axis parallel to the $Z$ axis of the crystal is introduced at the very centre of the nano-layer. As mentioned in Section 2.4.2.3, function CRACK_VOID_V_7_00 decides which atoms are to be removed from the perfect lattice by calculating whether each atom lies within the surface of the void. The surface is defined as an equation in 3-D space, and can be specified for any desired void geometry, provided it is fairly simple. A point is determined to lie inside the void if the result of a residual function is greater than or equal to zero. For the 7 Angstroms radius hole (which is a cylinder along the $Z$ axis) placed at the centre of the $15 \times 15 \times 1(54.225 \times 54.225 \times 3.615$ Angstroms $)$ nano-layer, the residual function is defined as:

$$
\text { Residual }=(7)^{2}-\left[\left(x-\frac{54.225}{2}\right)^{2}+\left(y-\frac{54.225}{2}\right)^{2}\right]
$$

It can easily be verified by putting in numbers that any atom on or within the cylindrical envelope of the hole produces a non-negative residual. Consequently, it is removed from the MD simulation. All other atoms are included in the simulation. Figure 9 and Figure 10 show two views of the copper nano-layer with the hole. The circles represent the atoms.

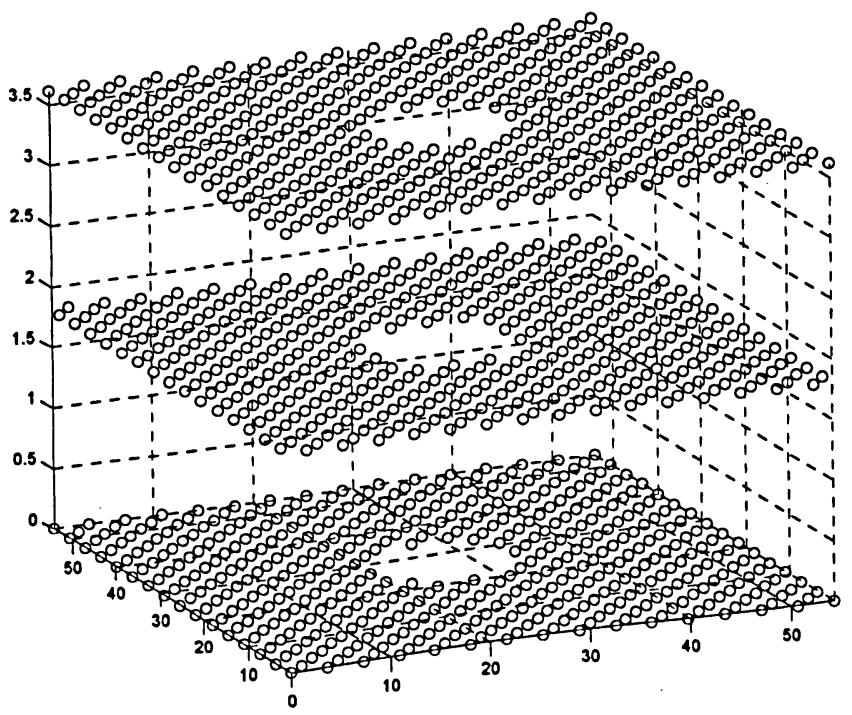

Figure 9 - Copper Nano-Layer with Cylindrical Void (View 1; Angstroms) 


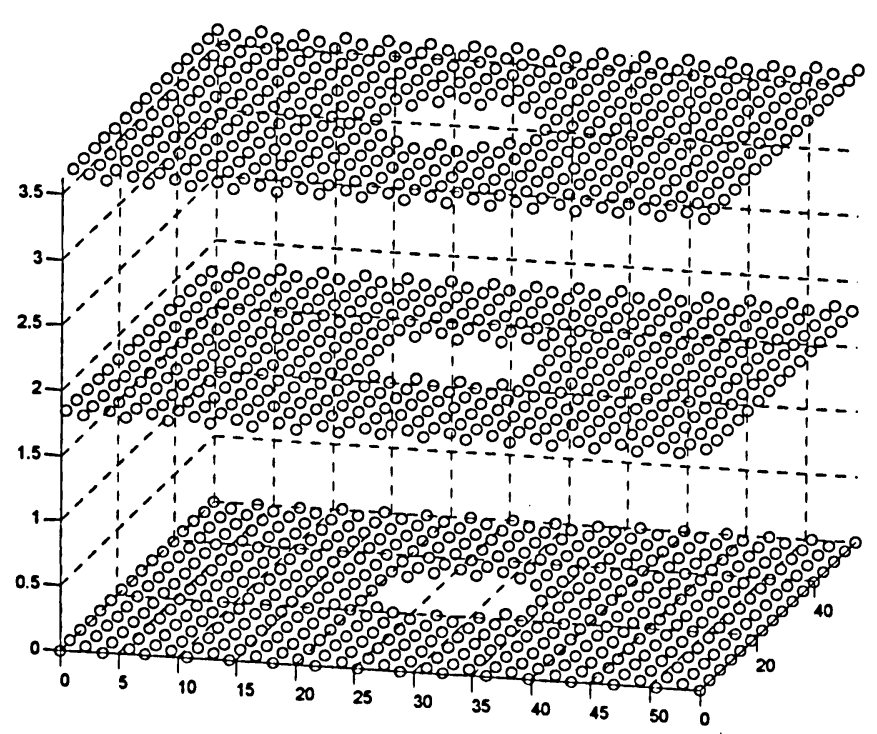

Figure 10 - Copper Nano-Layer with Cylindrical Void (View 2; Angstroms)

The nano-layer is then extended infinitely in the $\mathrm{Z}$ direction using PBCs in MD_V_7_00. Since the cut-off distance for the EAM potential developed for copper in Section 3.5 is just 6 Angstroms and there are $15 \times 15$ unit cells in the $\mathrm{XY}$ plane, there is no need to impose PBCs in the $\mathrm{X}$ and $\mathrm{Y}$ directions. Rather, the atoms on the outer edges are clamped rigid, since these atoms are not expected to move in any direction (on the average). This saves simulation time, while ensuring correct stresses for all atoms save those in the outermost 2 rows along the $\mathrm{X}$ and $\mathrm{Y}$ edges, which are of no consequence. Again, this was one of the reasons for extending the crystal lattice for 15 unit cells in the $\mathrm{X}$ and $\mathrm{Y}$ directions.

\subsubsection{Core MD Simulation Parameters}

The core parameters for the MD simulation are as follows: 


$\begin{array}{lll}\text { Material } & : & \text { Copper } \\ \text { Interatomic potential } & : & \text { Numerically Fit EAM Potential } \\ \text { Time steps } & : & 5000 \\ \text { Time increment } & : & 1 \mathrm{e}-14 \mathrm{sec} \\ \text { Damping } & : & \text { Yes }\end{array}$

Applied initial strain tensors (determined by MD_V_7_00 for desired far-field stress tensors):

$$
\begin{gathered}
\underline{\varepsilon}=\left[\begin{array}{lll}
0 & 0 & 0 \\
0 & 0 & 0 \\
0 & 0 & 0
\end{array}\right] \% \text { strain (reference case) } \\
\underline{\varepsilon}=\left[\begin{array}{ccc}
0.149026 & 0 & 0 \\
0 & -0.061871 & 0 \\
0 & 0 & -0.061871
\end{array}\right] \% \text { strain (stressed case) }
\end{gathered}
$$

Note: Atoms within void are first removed from the perfect crystal. Strain tensor is only applied afterwards.

Corresponding initial stress tensors:

$$
\begin{aligned}
& \underline{\sigma}=\left[\begin{array}{ccc}
0 & 0 & 0 \\
0 & 0 & 0 \\
0 & 0 & 0
\end{array}\right] \mathrm{MPa} \text { far-field/bulk (reference case) } \\
& \underline{\sigma}=\left[\begin{array}{ccc}
100 & 0 & 0 \\
0 & 0 & 0 \\
0 & 0 & 0
\end{array}\right] \mathrm{MPa} \text { far-field/bulk (stressed case) }
\end{aligned}
$$


Boundary conditions $\quad$ : $\quad$ Clamping along $\mathrm{X}$ and $\mathrm{Y}$, dynamic periodic boundary conditions (PBCs) along $\mathrm{Z}$

\subsubsection{Application 1 Results: Virial Stress Increment Plots}

The MD simulation took roughly 2 hours to run for each of the two cases (reference (base) and stressed). The atomic velocities after 5000 time steps were found to be less than $0.001 \mathrm{~m} / \mathrm{s}$. The maximum difference in positions between one time step and the next was about 1e-04 Angstroms, which was sufficient enough to warrant discontinuing the simulations.

Plots of stress increments (not absolute stresses, for reasons already discussed in Section 4.1) are shown in Figure 11 through Figure 16. The stress increments are the stresses of the reference MD simulation subtracted from the stresses of the stressed MD simulation.

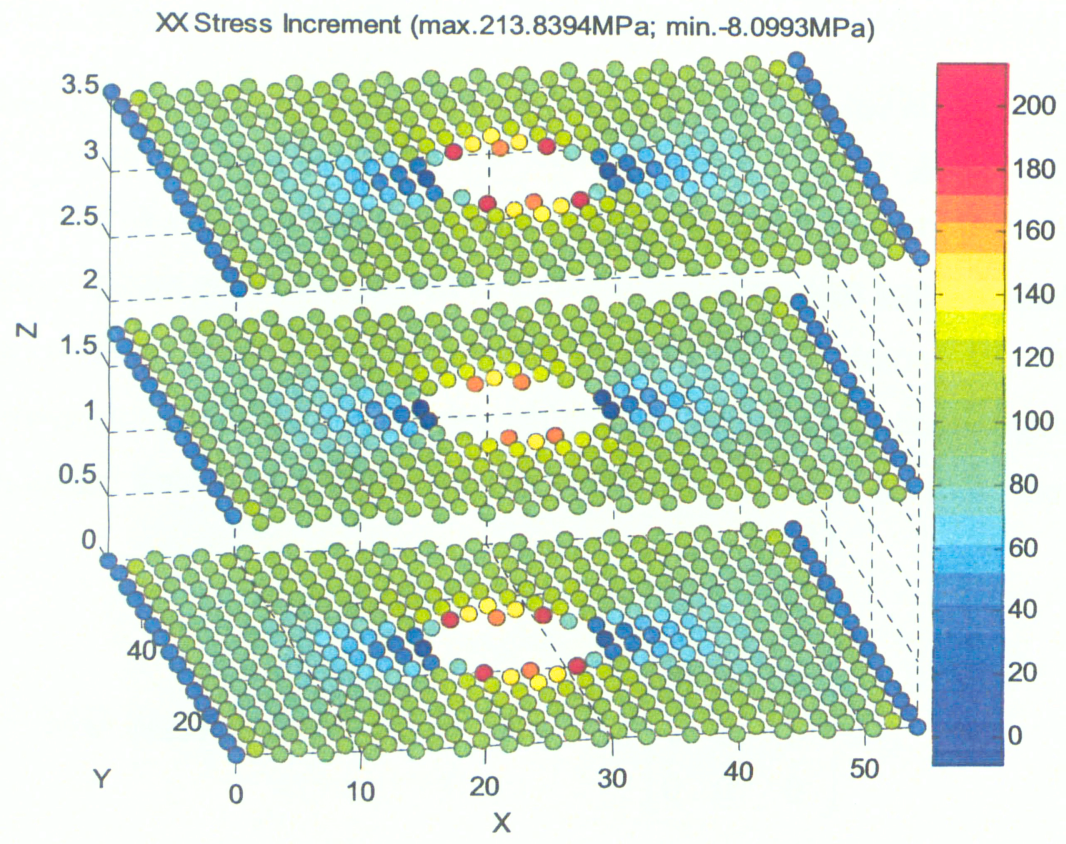

Figure 11 - Application 1: XX Stress Increment (MPa) 


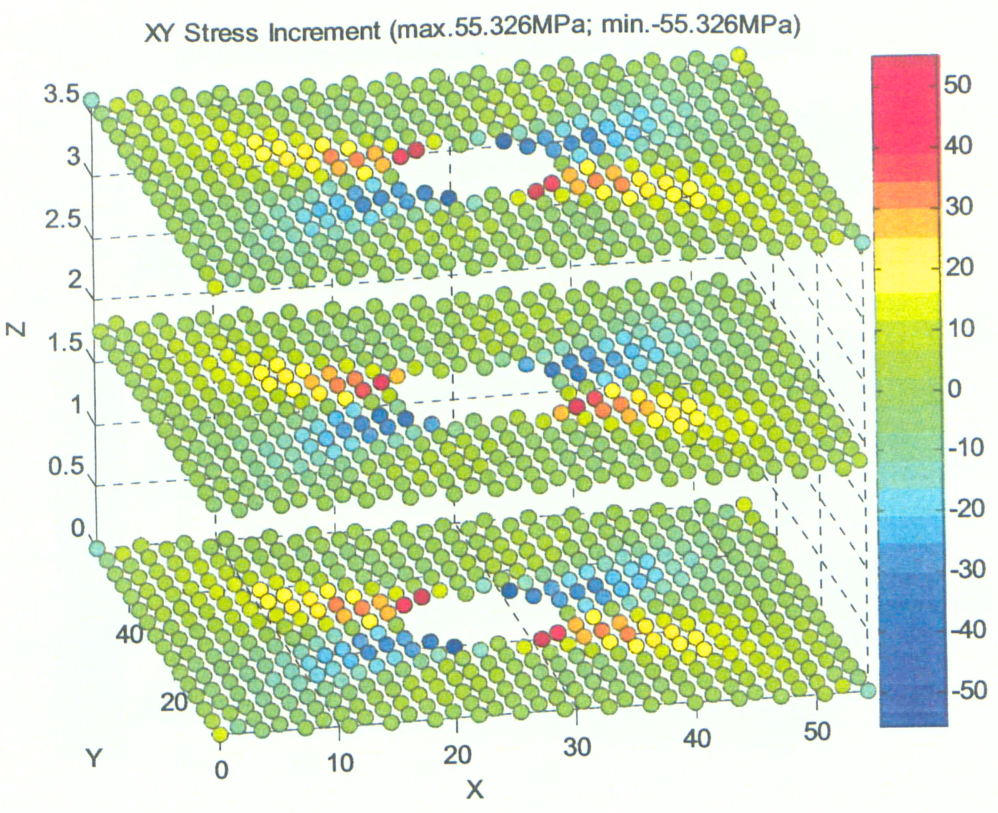

Figure 12 - Application 1: XY (YX) Stress Increment (MPa)

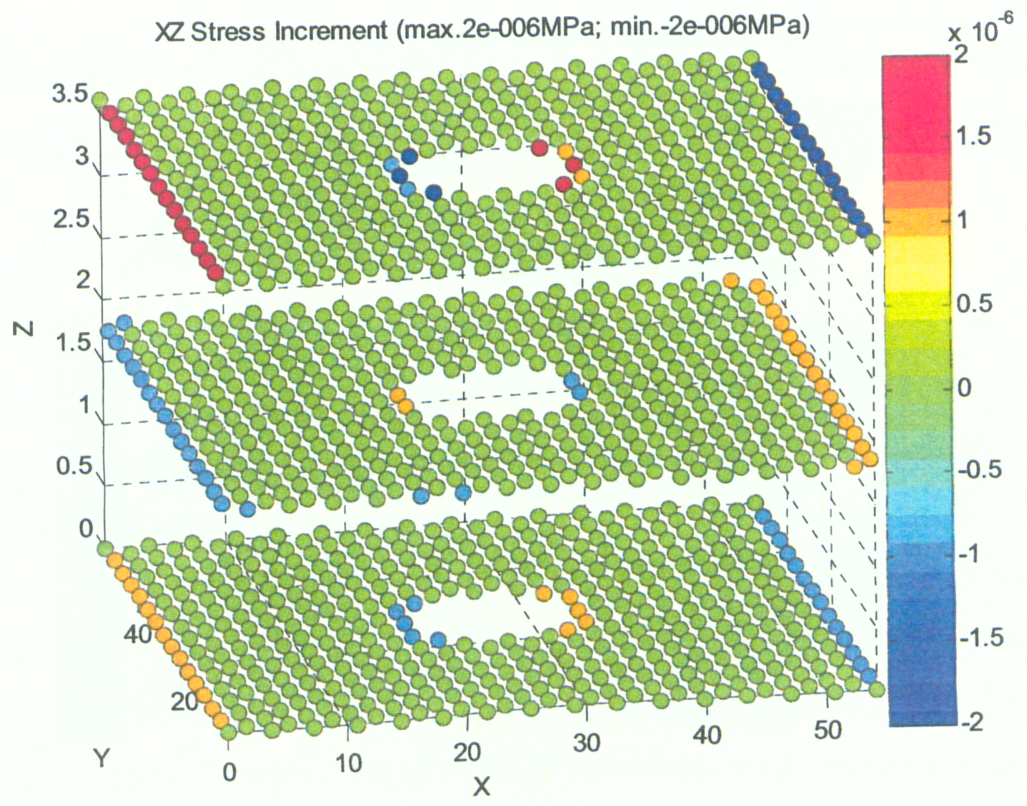

Figure 13 - Application 1: XZ (ZX) Stress Increment (MPa) 


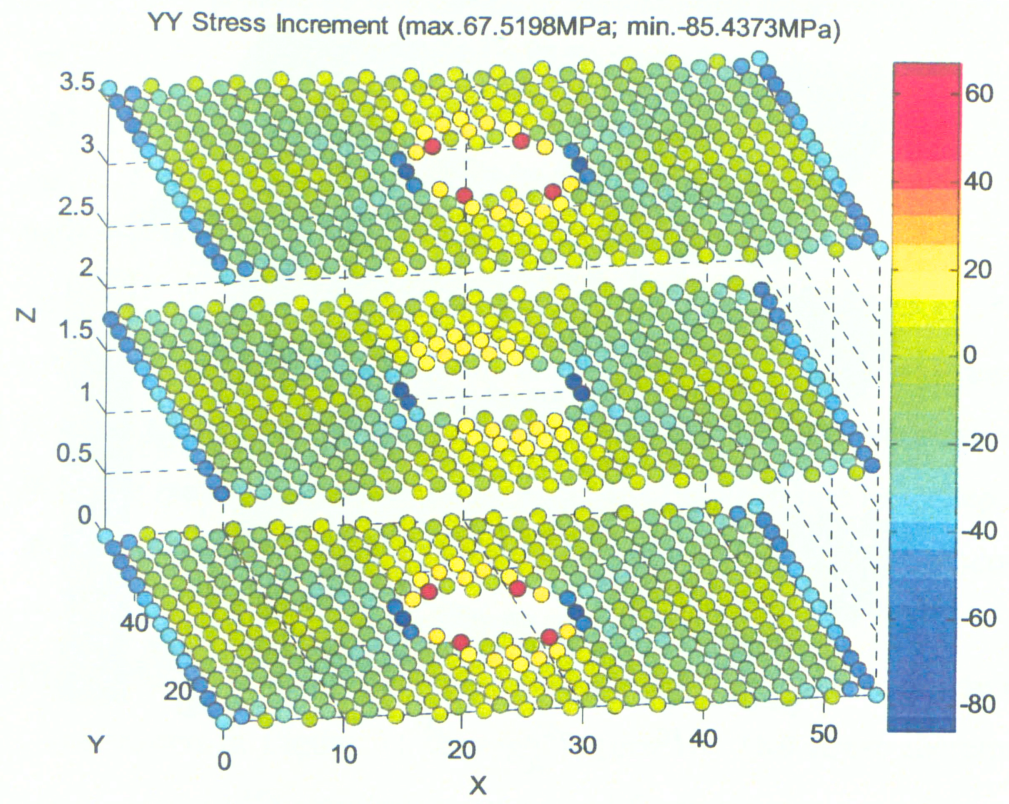

Figure 14 - Application 1: YY Stress Increment (MPa)

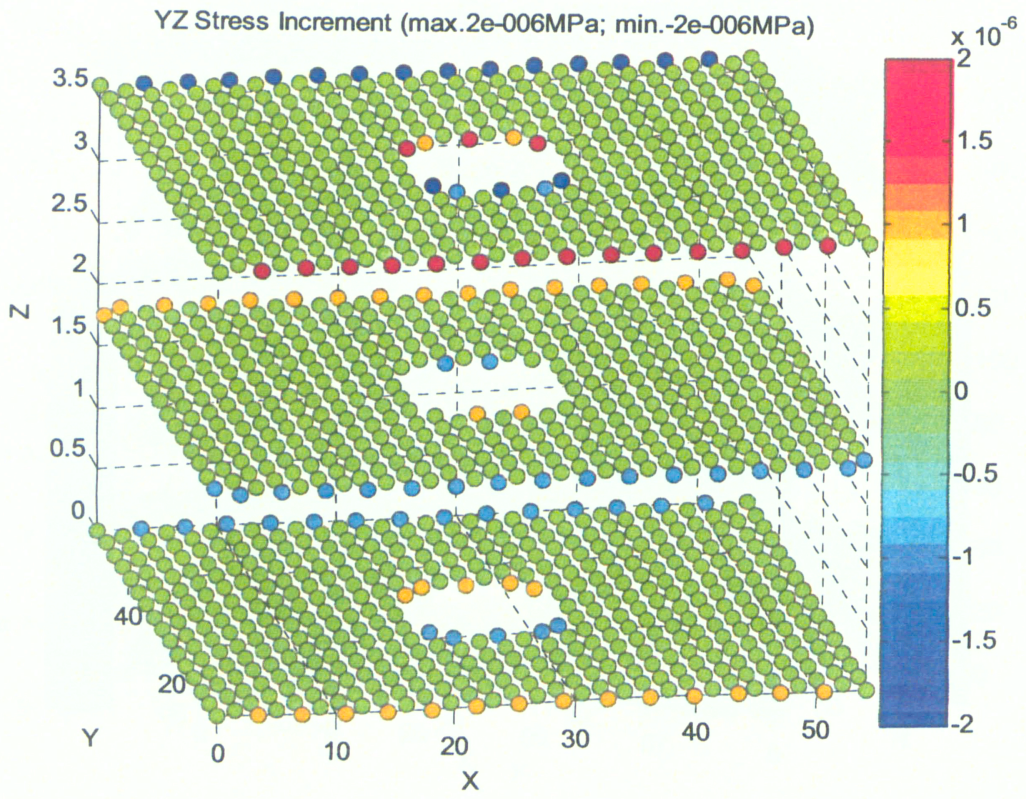

Figure 15 - Application 1: YZ (ZY) Stress Increment (MPa) 


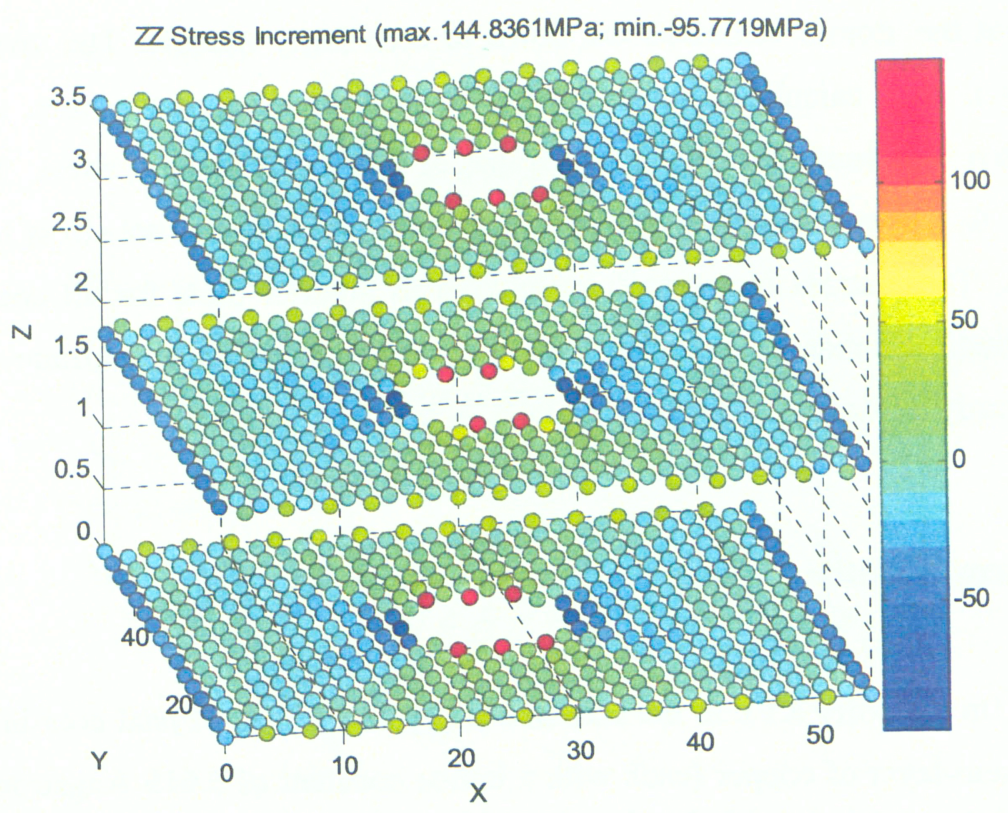

Figure 16 - Application 1: ZZ Stress Increment (MPa)

\subsection{Application 2: Elastically Stressed Infinite, Semi-Cylindrical Notch at the Free Surface of a Perfect Copper Crystal Lattice}

\subsubsection{Application Objective}

This application uses the copper EAM potential and D_V_7_00 to perform a Molecular Dynamics (MD) simulation of an infinite, semi-cylindrical notch at the free surface of a semi-infinite copper crystal lattice. The copper lattice with the notch in it is first generated in MD_V_7_00, and a strain tensor corresponding to a far-field (bulk) uniaxial stress of $100 \mathrm{MPa}$ in the $\mathrm{XX}$ direction is then applied to the whole lattice. The atoms are then allowed to relax through a damped MD simulation. Appropriate damping (defined according to convention as the product of a damping constant and the atomic velocities) 


\section{APPLICATION OF MOLECULAR DYNAMICS WITH THE EMBEDDED ATOM METHOD POTENTIAL}

ensures that the atomic velocities die down to zero fairly quickly. The virial stress on each atom is then calculated and recorded. A strain-free version is then simulated to calculate the base (undeformed) local stresses at zero far-field base stress. The uniaxial results are then subtracted from the reference results to yield the net stress increment of each atom. These stress increments are plotted as contour plots for all components of stress. Periodic Boundary Condition (PBC) is used to simulate the infinite surrounding lattice (already described in Section 2.4.3.4).

\subsubsection{Semi-Infinite Copper Lattice Specifications}

A $15 \times 8 \times 1$ (in the order XYZ in the standard [100]-[010]-[001] crystal coordinate system) unit cell nano-layer of copper (each with a lattice constant of 3.615 Angstroms, i.e. each at 293K) is first generated using MD_V_7_00. A 6 Angstroms radius semi-cylindrical notch with its axis parallel to the $Z$ axis of the crystal is introduced at the positive $Y$ surface of the nano-layer. The residual function for CRACK_VOID_V_7_00 is defined as:

$$
\text { Residual }=(6)^{2}-\left[\left(x-\frac{54.225}{2}\right)^{2}+(y-28.92)^{2}\right]
$$

It can easily be verified by putting in numbers that any atom on or within the semicylindrical envelope of the notch produces a non-negative residual. Consequently, it is removed from the MD simulation. All other atoms are included in the simulation. Figure 17 and Figure 18 show two views of the copper nano-layer with the surface notch.

The nano-layer is then extended infinitely in the $Z$ direction using PBCs. The atoms on the positive and negative $\mathrm{X}$ edges, and the negative $\mathrm{Y}$ edge alone (not the notched free surface), are clamped rigid. 


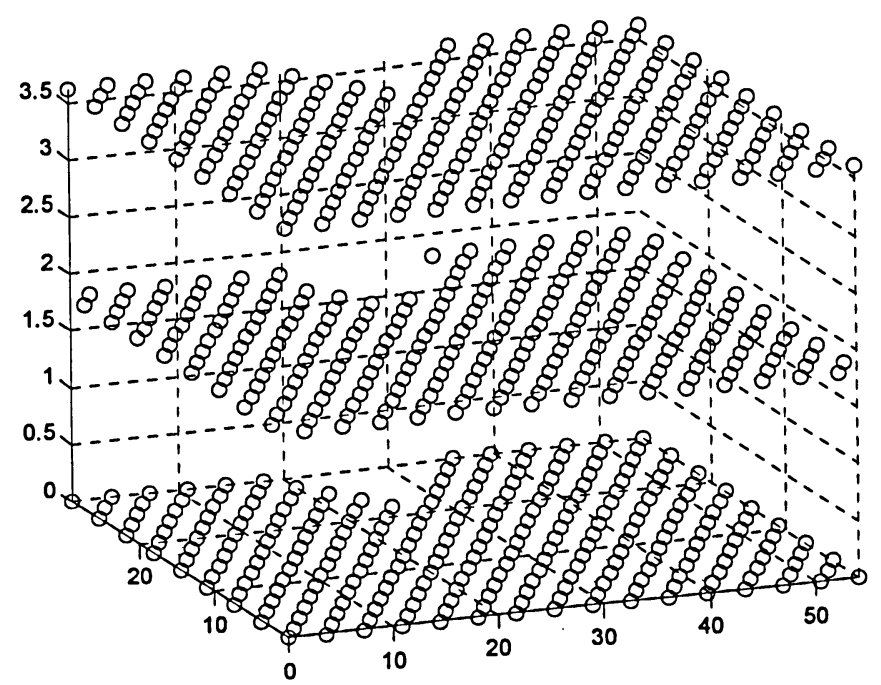

Figure 17 - Copper Nano-Layer with Surface Notch (View 1; Angstroms)

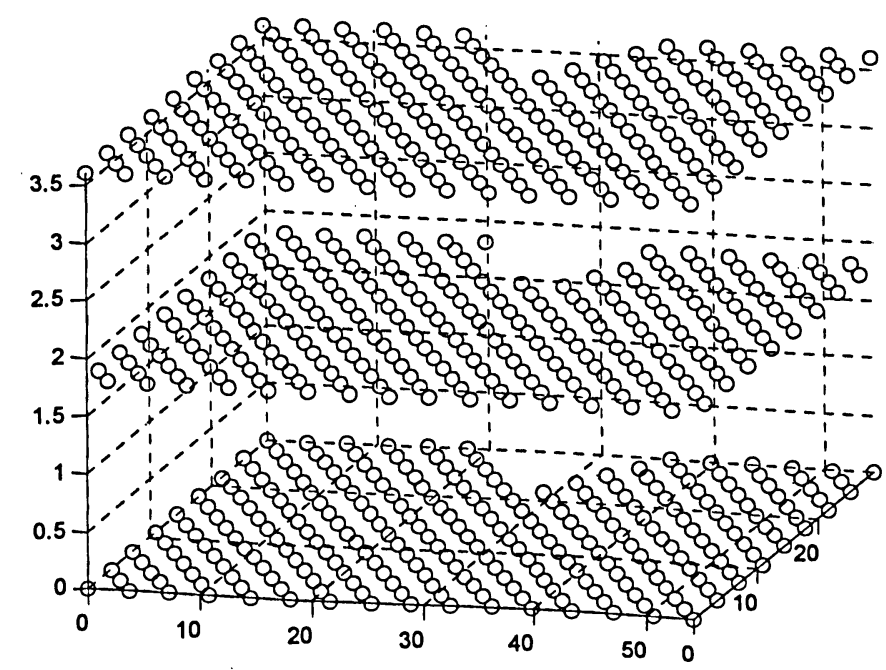

Figure 18 - Copper Nano-Layer with Surface Notch (View 2; Angstroms) 


\subsubsection{Core MD Simulation Parameters}

The core parameters for the MD simulation are as follows:

Material

Interatomic potential

Time steps

Time increment

Damping
: Copper

: Numerically Fit EAM Potential

: $\quad 5000$

: $\quad 1 \mathrm{e}-14 \mathrm{sec}$

: Yes

Applied initial strain tensors (determined by MD_V_7_00 for desired far-field stress tensors):

$$
\begin{gathered}
\underline{\varepsilon}=\left[\begin{array}{lll}
0 & 0 & 0 \\
0 & 0 & 0 \\
0 & 0 & 0
\end{array}\right] \% \text { strain (reference case) } \\
\underline{\varepsilon}=\left[\begin{array}{ccc}
0.149026 & 0 & 0 \\
0 & -0.061871 & 0 \\
0 & 0 & -0.061871
\end{array}\right] \% \text { strain (stressed case) }
\end{gathered}
$$

Note: Atoms within void are first removed from the perfect crystal. Strain tensor is only applied afterwards.

Corresponding initial stress tensors:

$$
\underline{\sigma}=\left[\begin{array}{lll}
0 & 0 & 0 \\
0 & 0 & 0 \\
0 & 0 & 0
\end{array}\right] \mathrm{MPa} \text { far-field/bulk (reference case) }
$$




$$
\underline{\sigma}=\left[\begin{array}{ccc}
100 & 0 & 0 \\
0 & 0 & 0 \\
0 & 0 & 0
\end{array}\right] \mathrm{MPa} \text { far-field/bulk (stressed case) }
$$

Boundary conditions : $\quad$ Clamping along $\mathrm{X}$ and $\mathrm{Y}$ (except positive $\mathrm{Y}$ ), dynamic periodic boundary conditions ( $\mathrm{PBCs}$ ) along $\mathrm{Z}$

\subsubsection{Application 2 Results: Stress Increment Plots}

The MD simulation took roughly 1 hour to run for each of the two cases (reference (base) and stressed). The atomic velocities after 5000 time steps were found to be less than $0.001 \mathrm{~m} / \mathrm{s}$. The maximum difference in positions between one time step and the next was about 1e-04 Angstroms, which was sufficient enough to warrant discontinuing the simulations.

Plots of stress increments (not absolute stresses, for reasons already discussed in Section 4.1) are shown in Figure 19 through Figure 24. The stress increments are the stresses of the reference MD simulation subtracted from the stresses of the stressed MD simulation. 


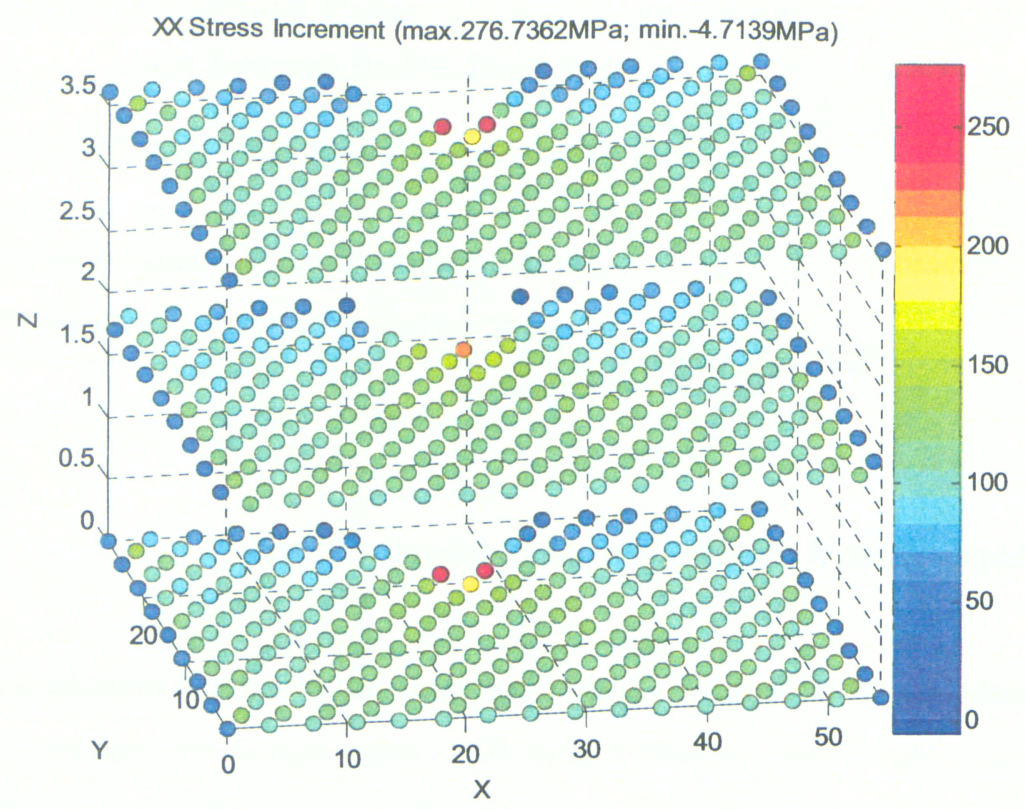

Figure 19 - Application 2: XX Stress Increment (MPa)

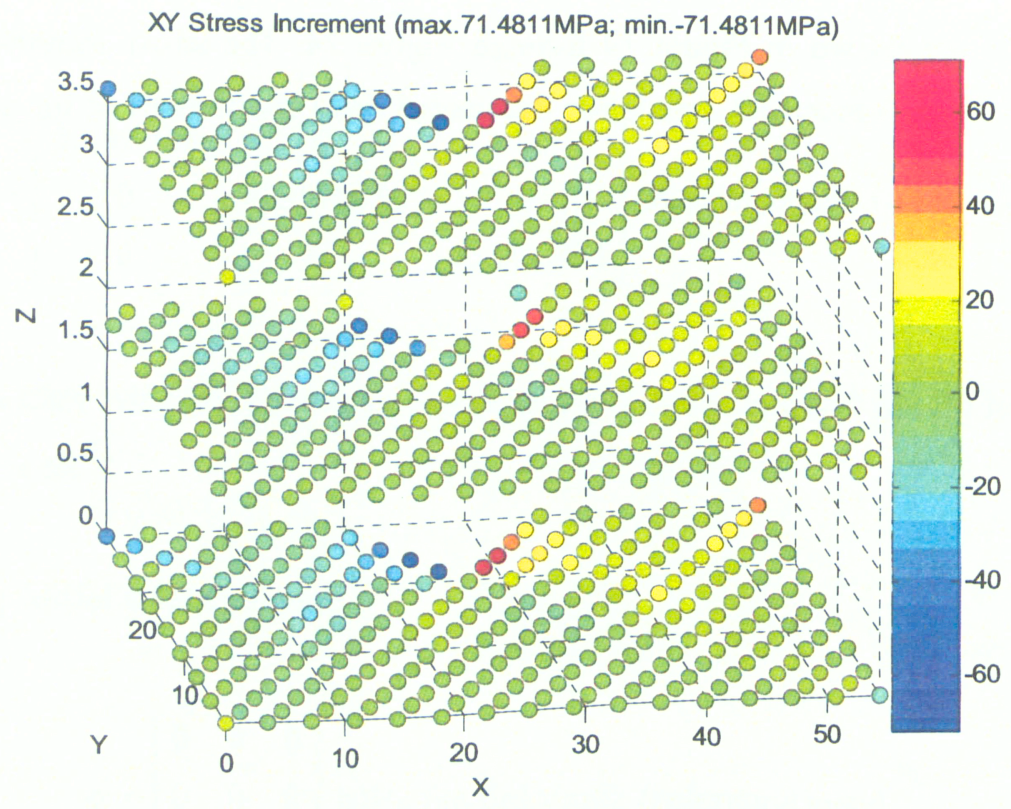

Figure 20 - Application 2: XY (YX) Stress Increment (MPa) 


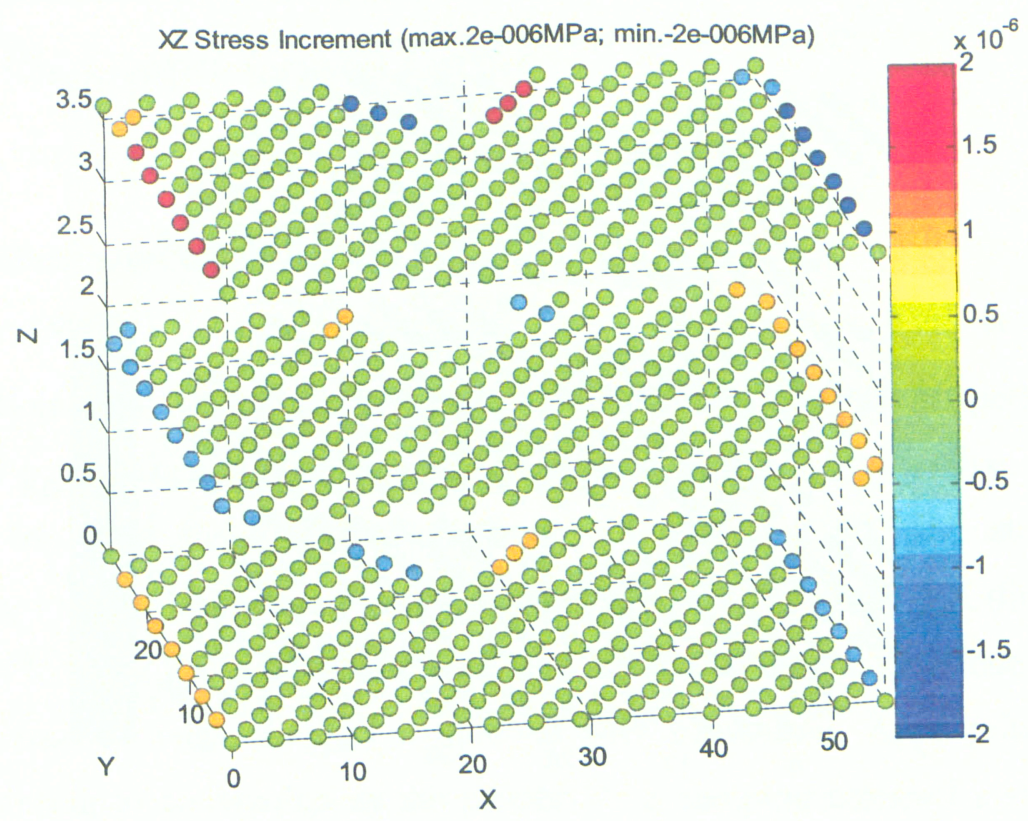

Figure 21 - Application 2: XZ (ZX) Stress Increment (MPa)

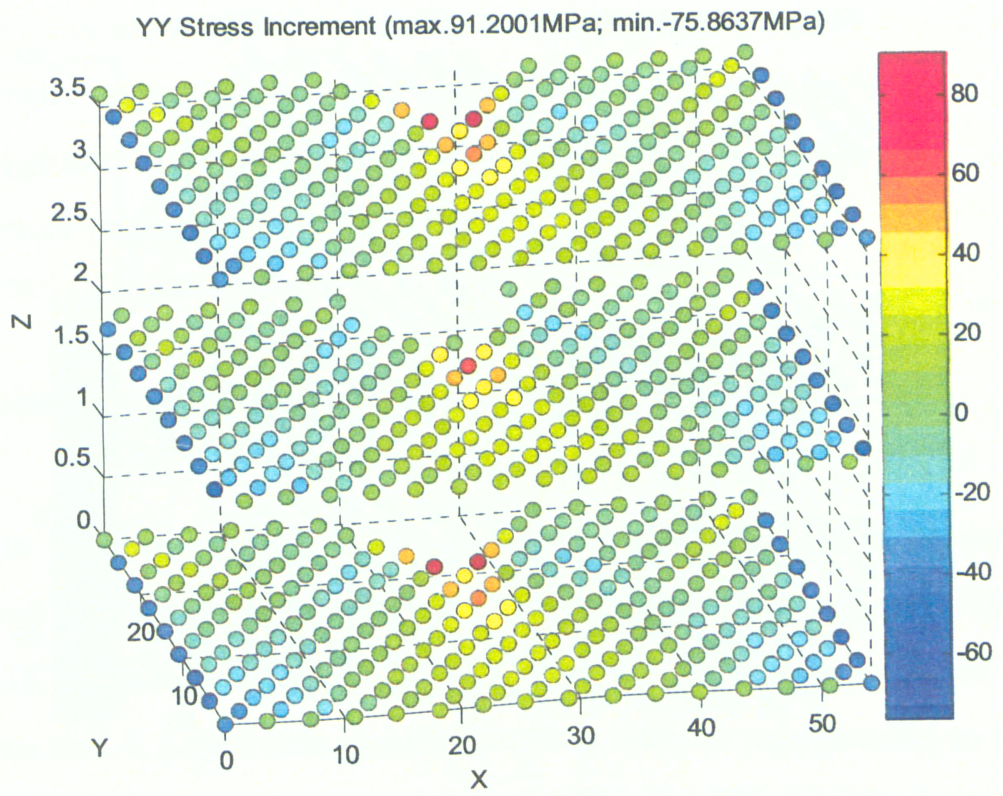

Figure 22 - Application 2: YY Stress Increment (MPa) 


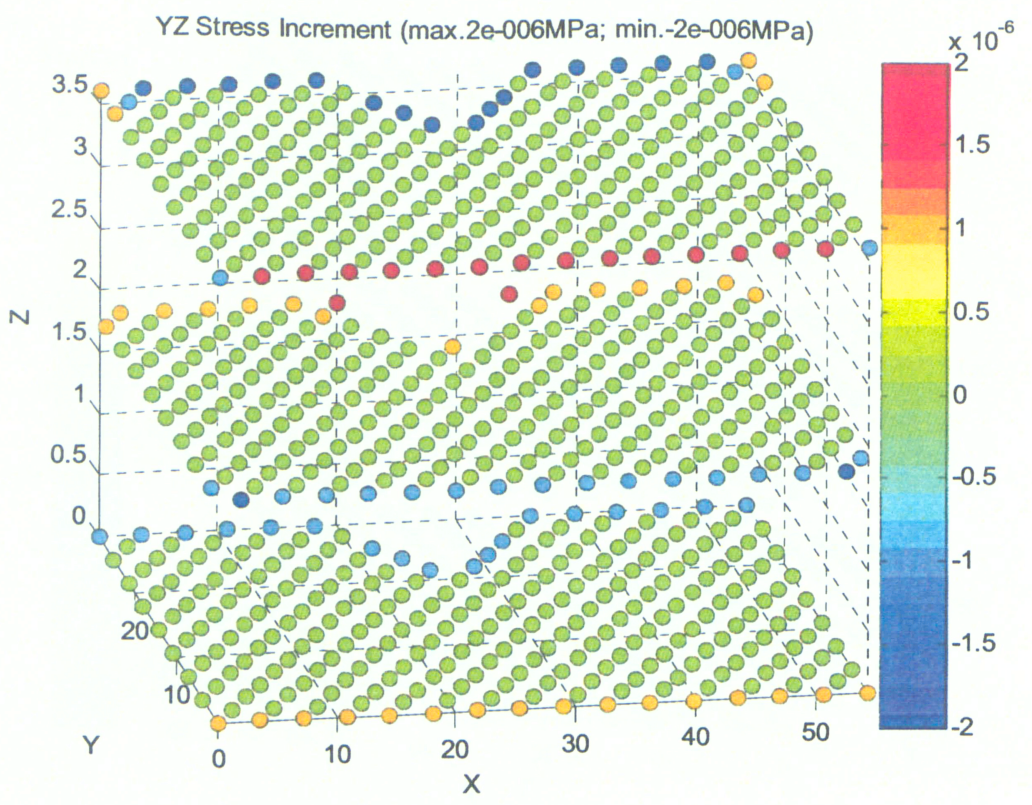

Figure 23 - Application 2: YZ (ZY) Stress Increment (MPa)

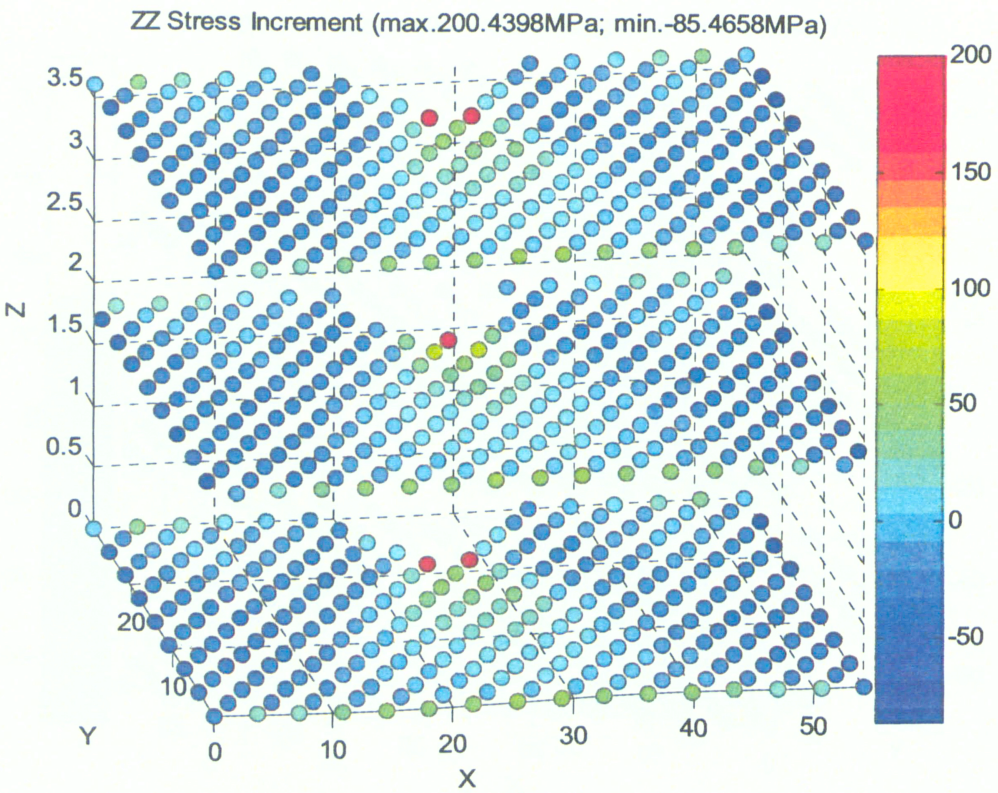

Figure 24 - Application 2: ZZ Stress Increment (MPa) 


\subsection{Application 3: Elastically Stressed Infinite, Elliptical Void in a Perfect Copper Crystal Lattice}

\subsubsection{Application Objective}

This application uses the copper EAM potential and MD_V_7_00 to perform a MD simulation of an infinite, elliptical void in an infinite copper crystal lattice. The copper lattice with the void in it is first generated in MD_V_7_00, and strain tensors corresponding to a far-field (bulk) uniaxial stress of $100 \mathrm{MPa}$ in the XX direction first, and $100 \mathrm{MPa}$ in the YY direction next, are then applied to the whole lattice. The atoms are then allowed to relax through a damped MD simulation. Appropriate damping (defined according to convention as the product of a damping constant and the atomic velocities) ensures that the atomic velocities die down to zero fairly quickly. The virial stress on each atom is then calculated and recorded. A strain-free version is then simulated to calculate the base (undeformed) local stresses at zero far-field base stress. The uniaxial results are then subtracted from the reference results to yield the net stress increment of each atom. These stress increments are plotted as contour plots for all components of stress. Periodic Boundary Condition (PBC) is used to simulate the infinite surrounding lattice (already described in Section 2.4.3.4).

\subsubsection{Infinite Copper Lattice Specifications}

A $15 \times 15 \times 1$ (in the order $\mathrm{XYZ}$ in the standard [100]-[010]-[001] crystal coordinate system) unit cell nano-layer of copper (each with a lattice constant of 3.615 Angstroms, i.e. each at 293K) is first generated using MD_V_7_00. An elliptical void of 7 Angstroms semi-major axis and 4 Angstroms semi-minor axis with its axis parallel to the $Z$ axis of the crystal is introduced at the centre of the $15 \times 15 \times 1$ ( $54.225 \times 54.225 \times 3.615$ Angstroms) nano-layer. The residual function for CRACK_VOID_V_7_00 is defined as: 


$$
\text { Residual }=(1)^{2}-\left[\left\{\frac{\left(x-\frac{54.225}{2}\right)}{7}\right\}^{2}+\left\{\frac{\left(y-\frac{54.225}{2}\right)}{4}\right\}\right]^{2}
$$

It can easily be verified by putting in numbers that any atom on or within the elliptical envelope of the void produces a non-negative residual. Consequently, it is removed from the MD simulation. All other atoms are included in the simulation. Figure 25 and Figure 26 show two views of the copper nano-layer with the surface notch.

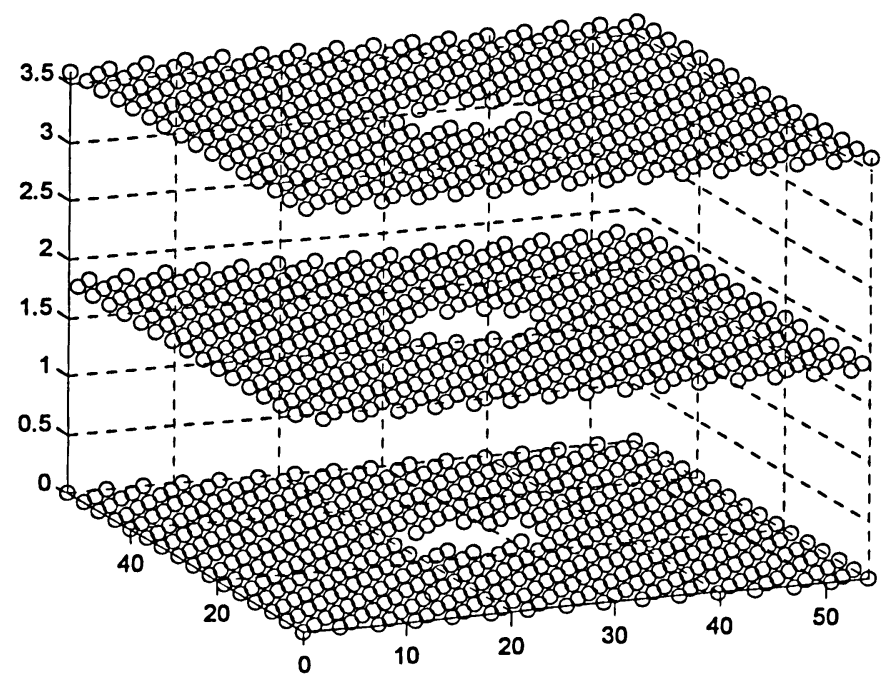

Figure 25 - Copper Nano-Layer with Elliptical Void (View 1; Angstroms) 


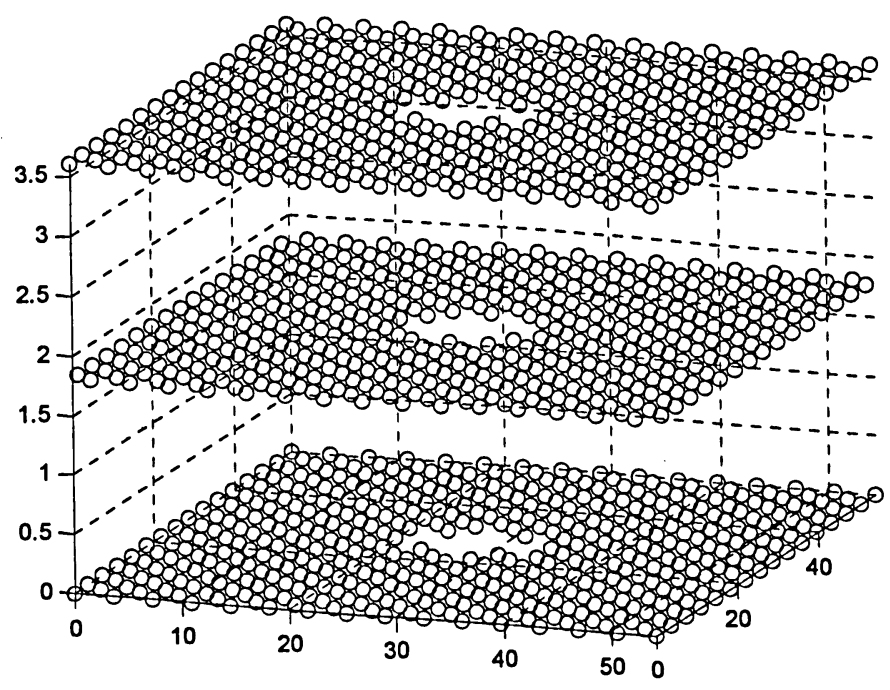

Figure 26 - Copper Nano-Layer with Elliptical Void (View 2; Angstroms)

The nano-layer is then extended infinitely in the $\mathrm{Z}$ direction using PBCs. The atoms on the positive and negative $\mathrm{X}$ edges, and the positive and negative $\mathrm{Y}$ edges, are clamped rigid.

\subsubsection{Core MD Simulation Parameters}

The core parameters for the MD simulation are as follows:

$\begin{array}{lll}\text { Material } & : & \text { Copper } \\ \text { Interatomic potential } & : & \text { Numerically Fit EAM Potential } \\ \text { Time steps } & : & 5000 \\ \text { Time increment } & : & 1 \mathrm{e}-14 \mathrm{sec} \\ \text { Damping } & : & \text { Yes }\end{array}$

Applied initial strain tensors (determined by MD_V_7_00 for desired far-field stress tensors): 


$$
\begin{gathered}
\underline{\varepsilon}=\left[\begin{array}{lll}
0 & 0 & 0 \\
0 & 0 & 0 \\
0 & 0 & 0
\end{array}\right] \% \text { strain (reference case) } \\
\underline{\varepsilon}=\left[\begin{array}{ccc}
0.149026 & 0 & 0 \\
0 & -0.061871 & 0 \\
0 & 0 & -0.061871
\end{array}\right] \% \text { strain (XX stressed case) } \\
\underline{\varepsilon}=\left[\begin{array}{ccc}
-0.061871 & 0 & 0 \\
0 & 0.149026 & 0 \\
0 & 0 & -0.061871
\end{array}\right] \% \text { strain (YY stressed case) }
\end{gathered}
$$

Note: Atoms within void are first removed from the perfect crystal. Strain tensor is only applied afterwards.

Corresponding initial stress tensors:

$$
\begin{gathered}
\underline{\sigma}=\left[\begin{array}{lll}
0 & 0 & 0 \\
0 & 0 & 0 \\
0 & 0 & 0
\end{array}\right] \mathrm{MPa} \text { far-field/bulk (reference case) } \\
\underline{\sigma}=\left[\begin{array}{ccc}
100 & 0 & 0 \\
0 & 0 & 0 \\
0 & 0 & 0
\end{array}\right] \mathrm{MPa} \text { far-field/bulk (XX stressed case) } \\
\underline{\sigma}=\left[\begin{array}{ccc}
0 & 0 & 0 \\
0 & 100 & 0 \\
0 & 0 & 0
\end{array}\right] \mathrm{MPa} \text { far-field/bulk (YY stressed case) }
\end{gathered}
$$


Boundary conditions $\quad$ : Clamping along $\mathrm{X}$ and $\mathrm{Y}$, dynamic periodic boundary conditions (PBCs) along $\mathrm{Z}$

\subsubsection{Application 3 Results: Stress Increment Plots}

The MD simulation took roughly 2 hours to run for each of the three cases (reference (base) and two stressed). The atomic velocities after 5000 time steps were found to be less than $0.001 \mathrm{~m} / \mathrm{s}$. The maximum difference in positions between one time step and the next was about 1e-04 Angstroms, which was sufficient enough to warrant discontinuing the simulations.

Plots of stress increments (not absolute stresses, for reasons already discussed in Section 4.1) are shown in Figure 27 through Figure 38. The stress increments are the stresses of the reference MD simulation subtracted from the stresses of the stressed MD simulation.

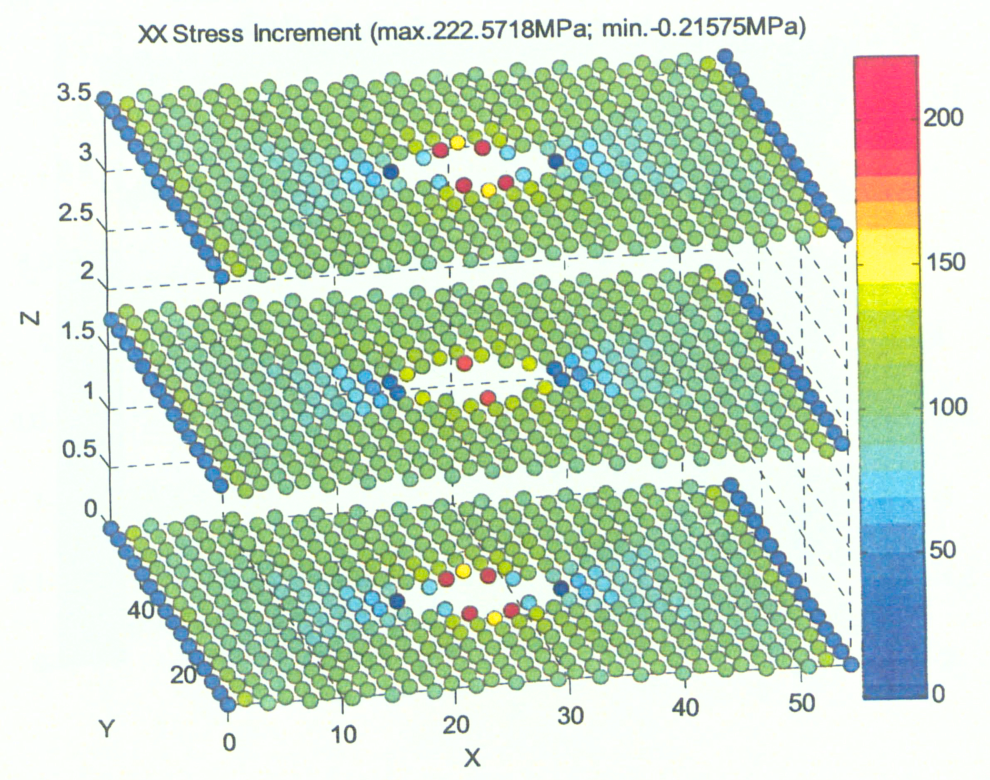

Figure 27 - Application 3: XX Stress Increment (MPa) for 100MPa XX Applied Far-Field Stress 


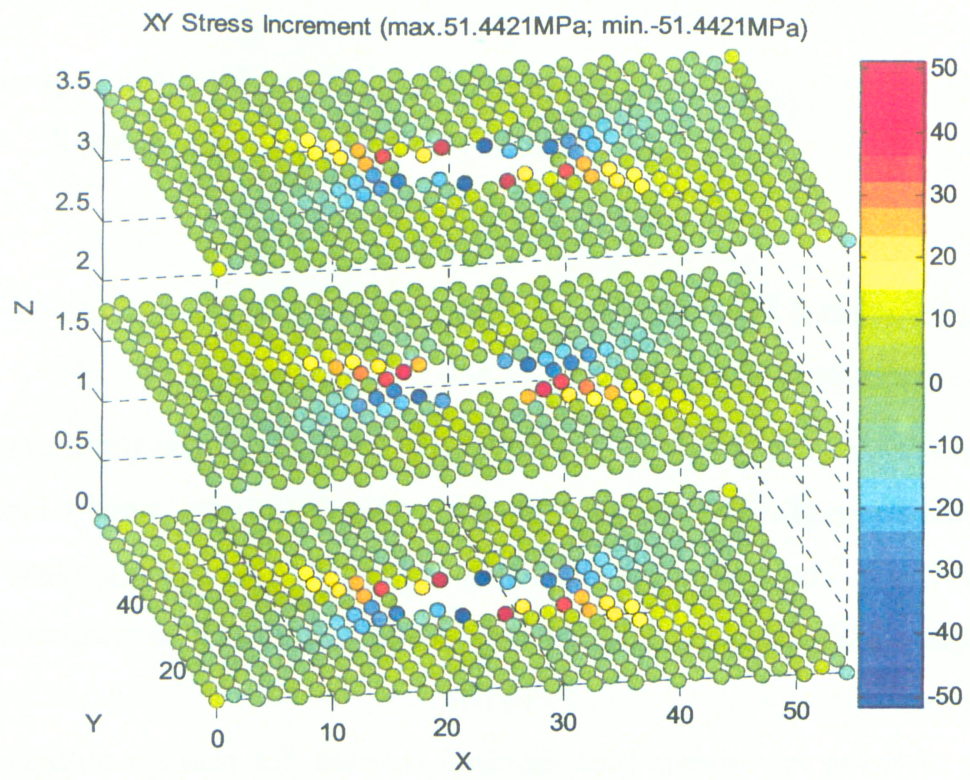

Figure 28 - Application 3: XY (YX) Stress Increment (MPa) for 100MPa XX Applied Far-Field Stress

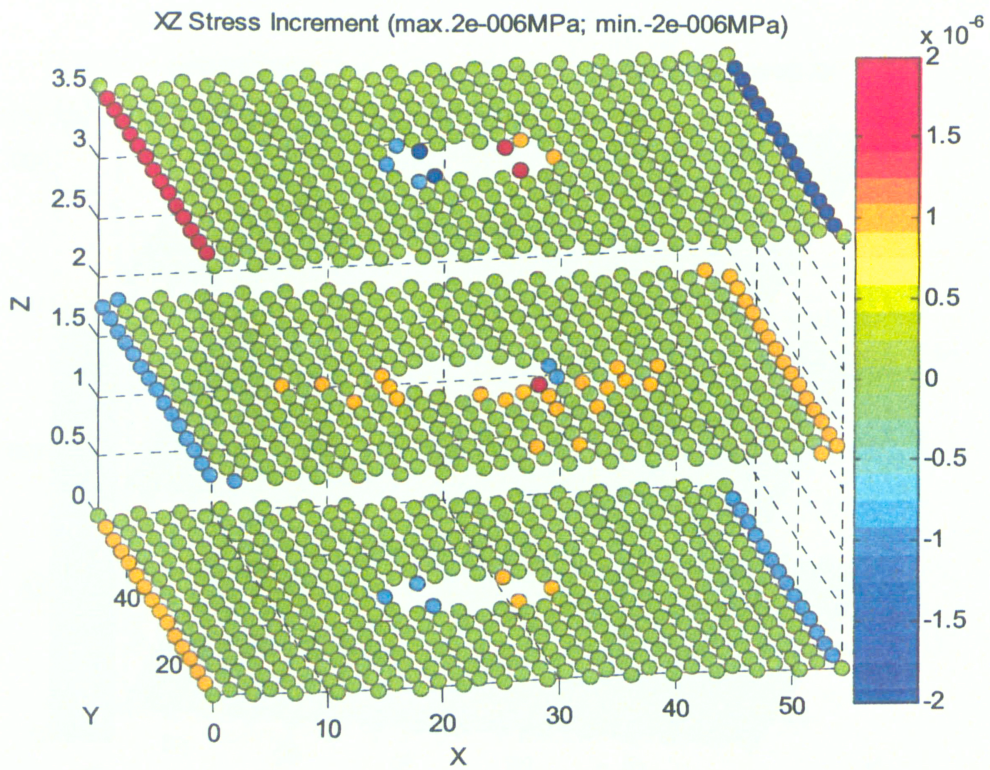

Figure 29 - Application 3: XZ (ZX) Stress Increment (MPa) for 100MPa XX Applied Far-Field Stress 


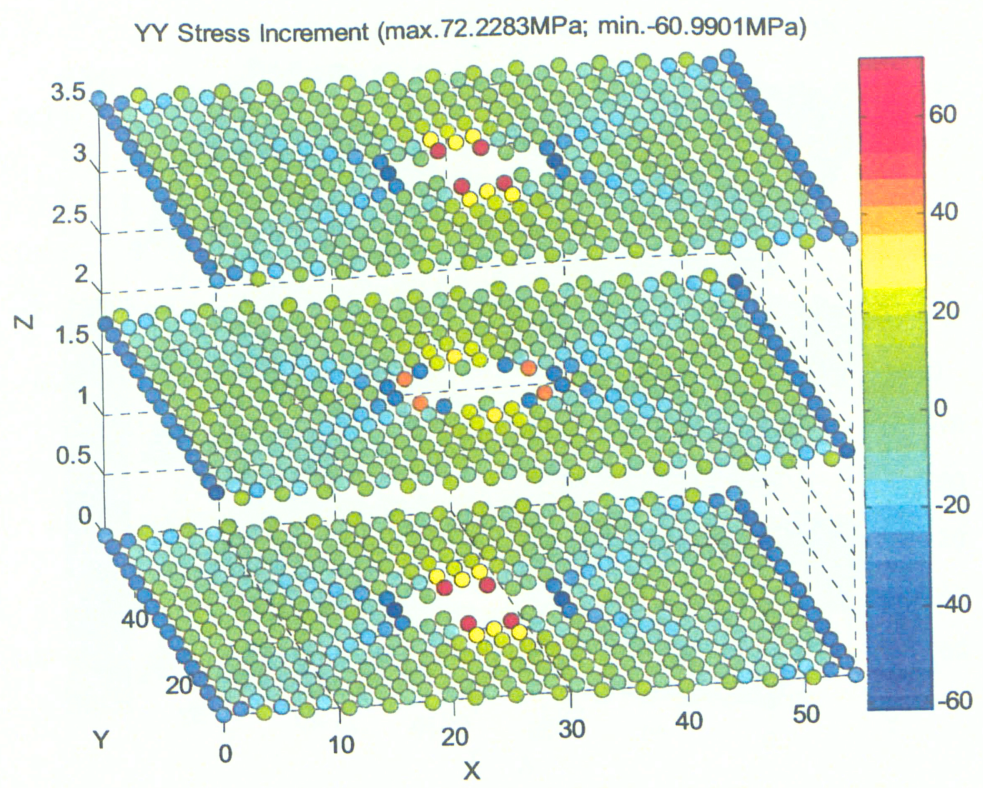

Figure 30 - Application 3: YY Stress Increment (MPa) for 100MPa XX Applied Far-Field Stress

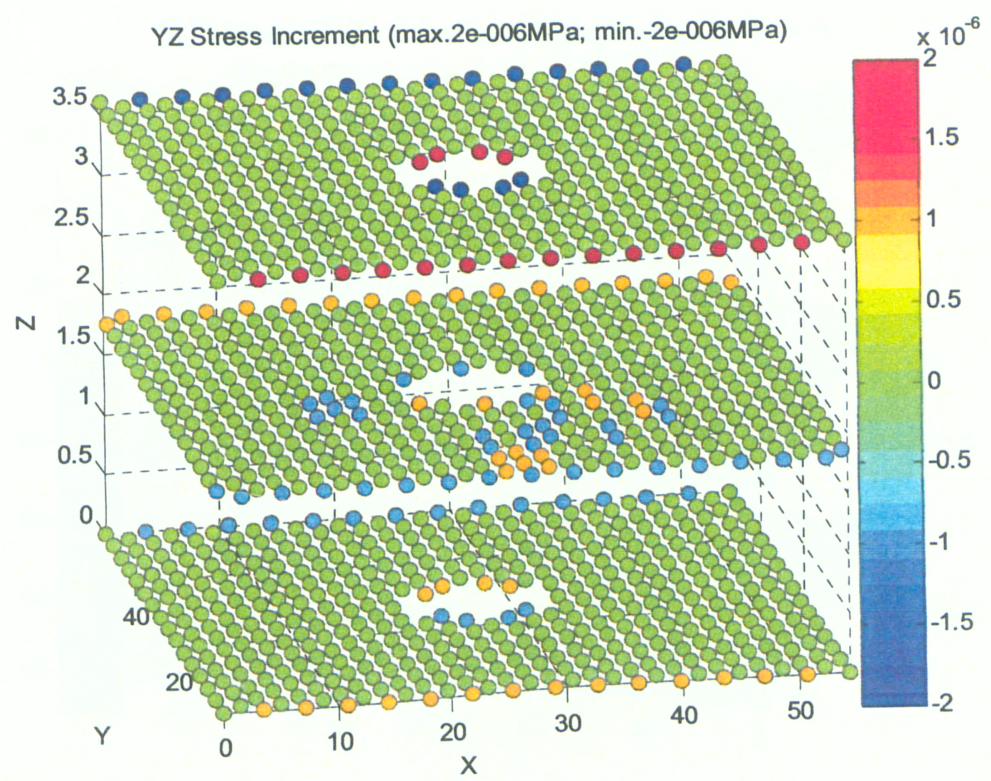

Figure 31 - Application 3: YZ (ZY) Stress Increment (MPa) for 100MPa XX Applied Far-Field Stress 


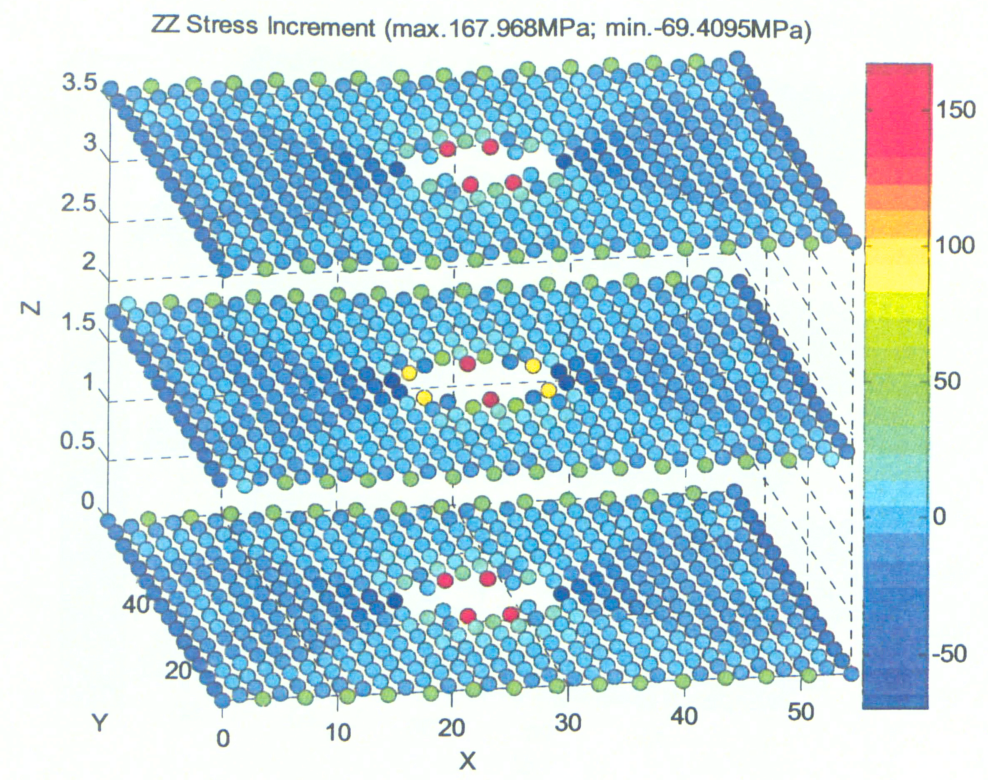

Figure 32 - Application 3: ZZ Stress Increment (MPa) for 100MPa XX Applied Far-Field Stress

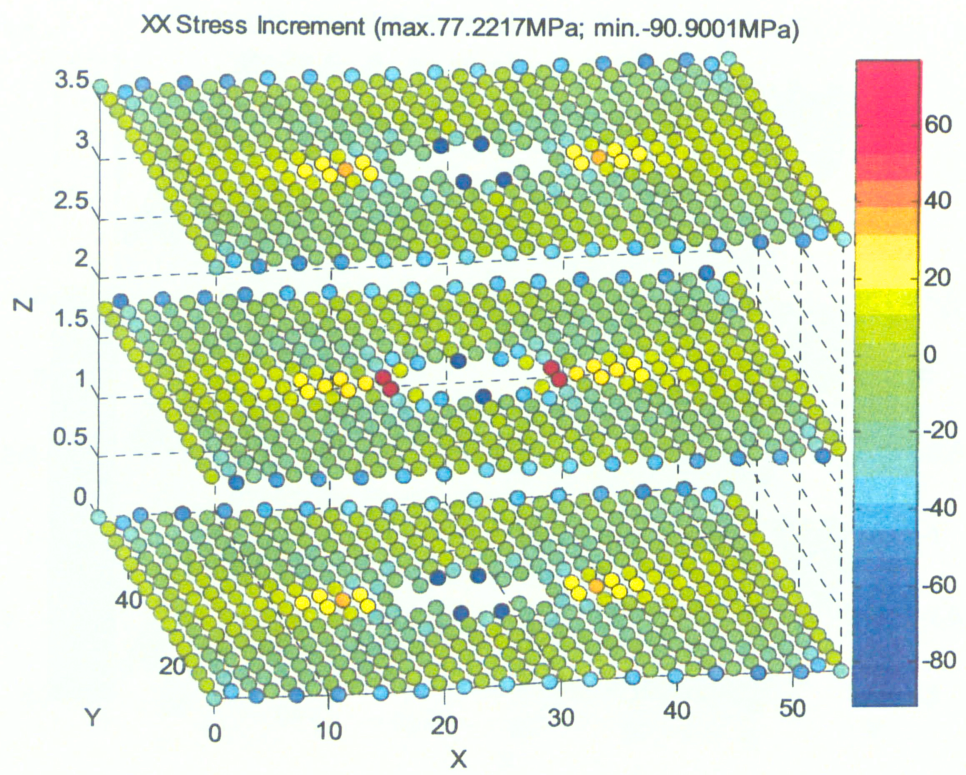

Figure 33 - Application 3: XX Stress Increment (MPa) for 100MPa YY Applied Far-Field Stress 


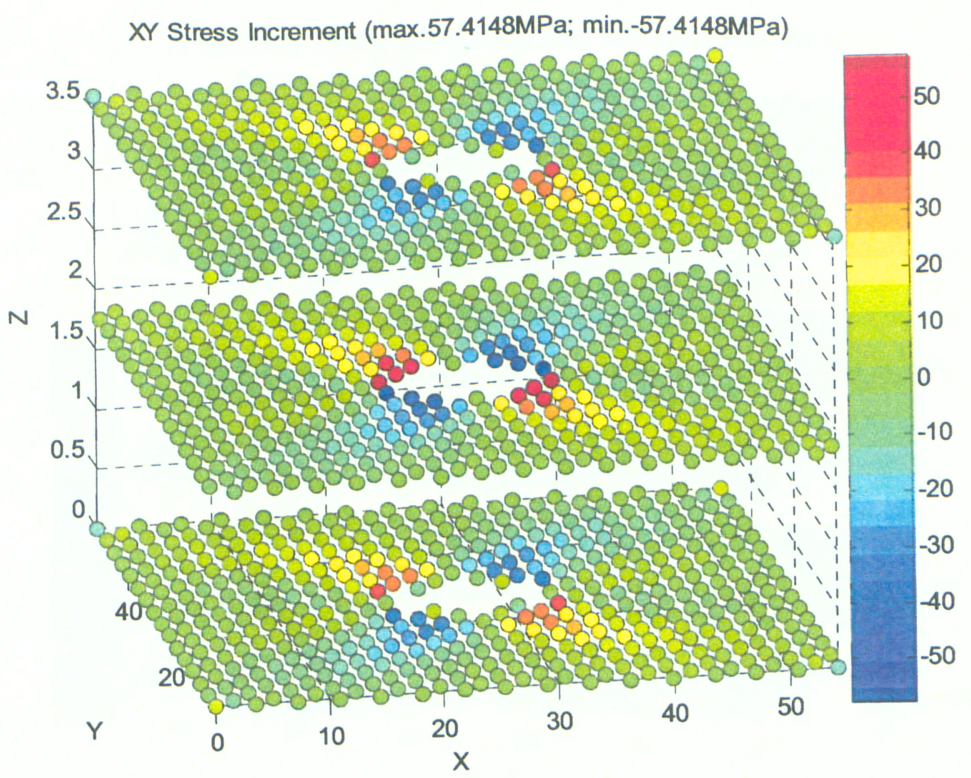

Figure 34 - Application 3: XY (YX) Stress Increment (MPa) for 100MPa YY Applied Far-Field Stress

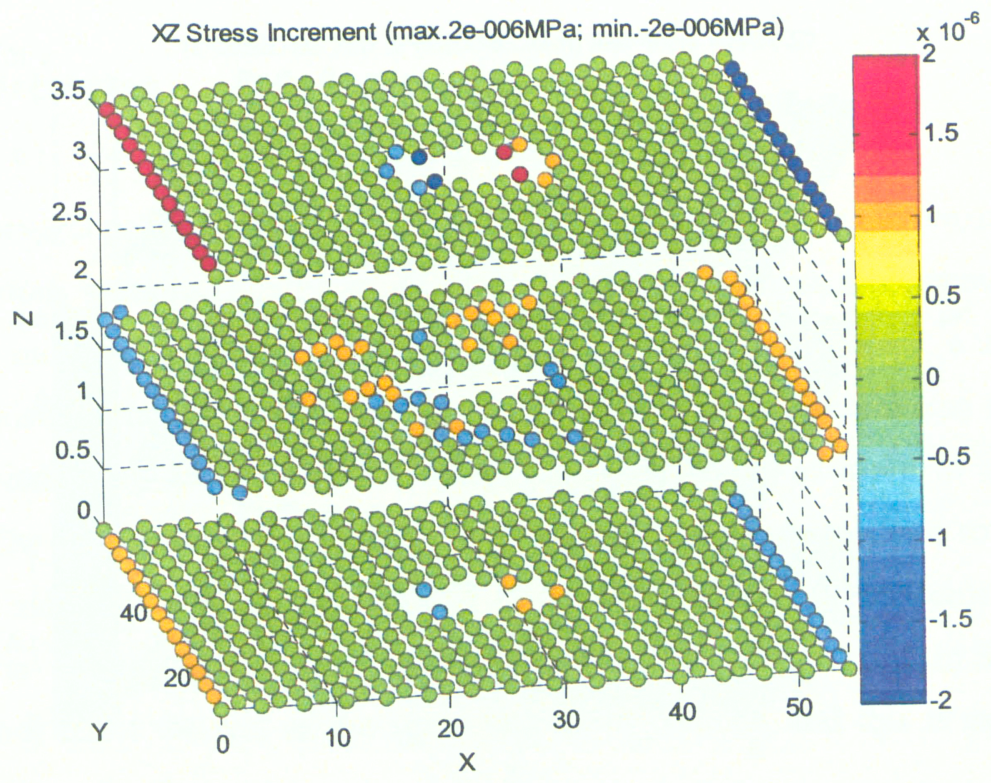

Figure 35 - Application 3: XZ (ZX) Stress Increment (MPa) for 100MPa YY Applied Far-Field Stress 


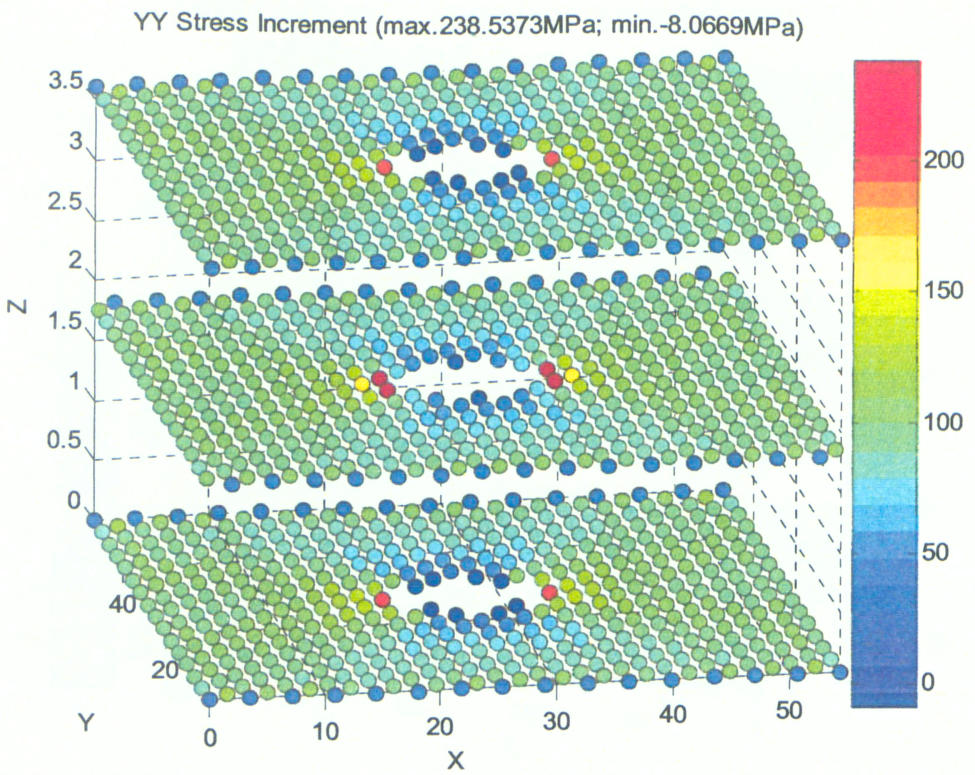

Figure 36 - Application 3: YY Stress Increment (MPa) for 100MPa YY Applied Far-Field Stress

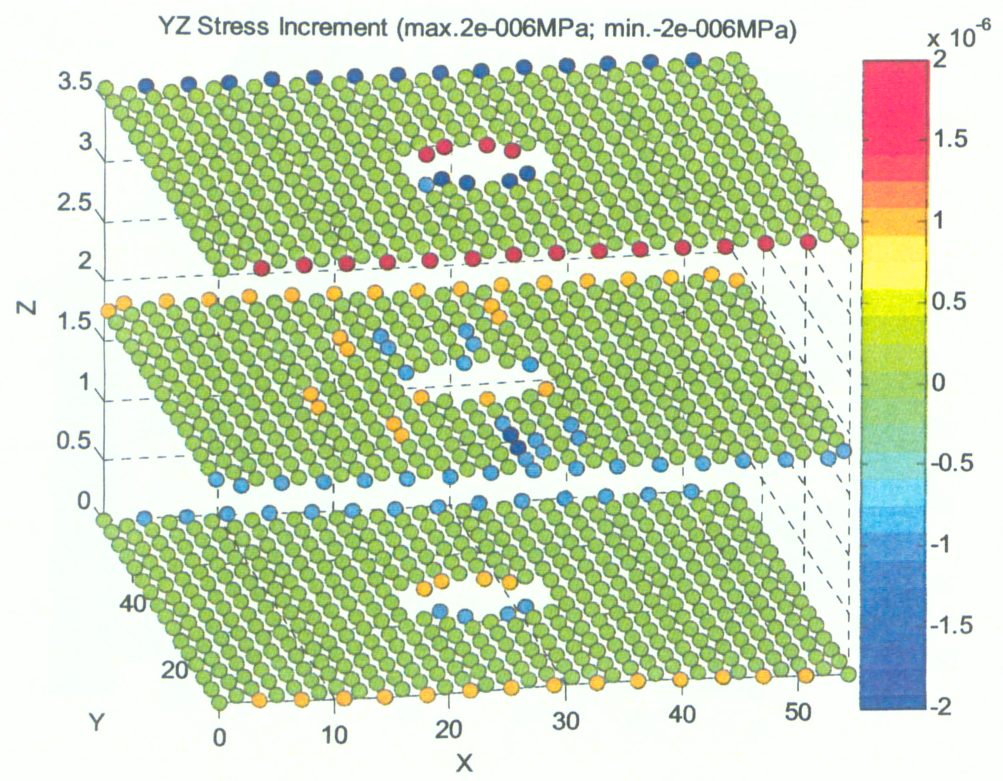

Figure 37 - Application 3: YZ (ZY) Stress Increment (MPa) for 100MPa YY Applied Far-Field Stress 


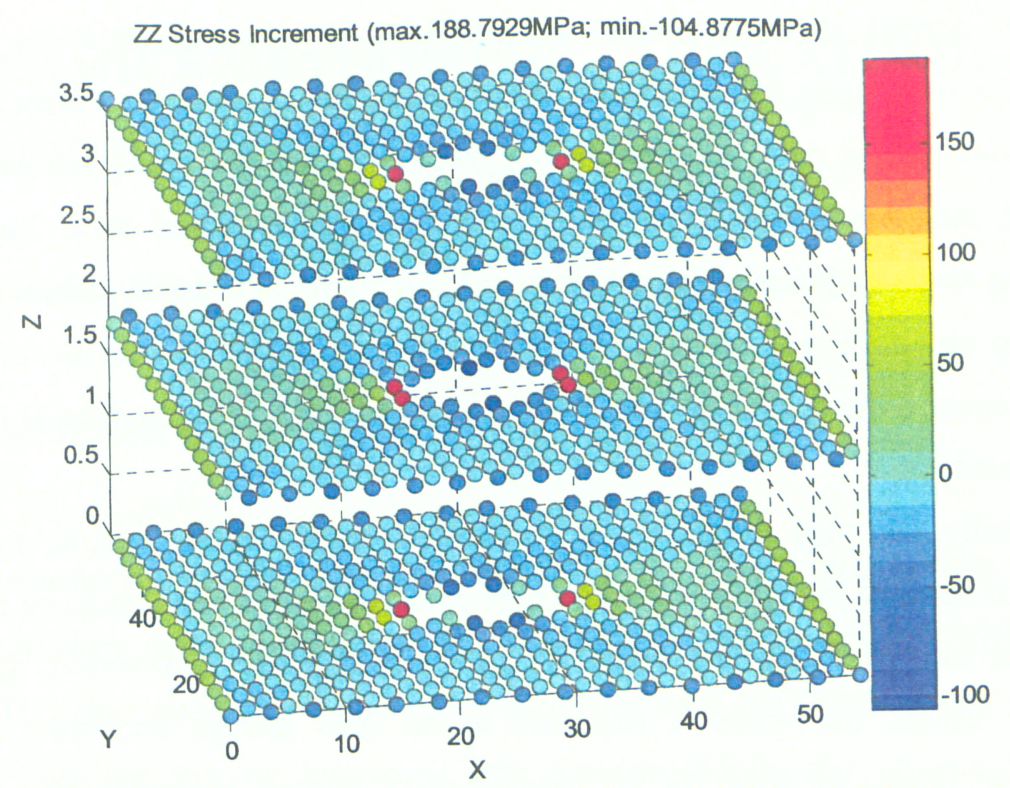

Figure 38 - Application 3: ZZ Stress Increment (MPa) for 100MPa YY Applied Far-Field Stress

\subsection{Discussion}

There are several points to be noted from the contour plots of stress increments presented in the previous sections. Firstly, in all cases, the far-field stress increments (i.e. stress increments on atoms sufficiently far away from the void) are as required: a uniaxial state of stress exists in the far-field for all cases. The magnitude of the uniaxial stress is 100 MPa far-field in all cases while all other stresses are zero or close to zero, just as required. The only exceptions to this are the atoms in the outermost two rows of atoms (i.e. atoms along the clamped edges). The stress increments on these atoms are not 100 MPa uniaxial because of the clamped boundary conditions. As discussed in Section 4.2.2, this does not affect the rest of the stress increment contour, and this is evident in the contour plots.

Secondly, the stress increment patterns (and not the stress increments themselves) show certain similarities to classical theory of elasticity results. For instance, there are 


\section{APPLICATION OF MOLECULAR DYNAMICS WITH THE EMBEDDED ATOM METHOD POTENTIAL}

regions of normal stress increment concentration at classical positions around the cylindrical void (Figure 11 and Figure 14). The XY shear stress increment peaks at the $45^{\circ}$ locations around the void (Figure 12), just as in classical theory of elasticity. That being said, there are several differences, which are to be expected since classical theory of elasticity deals with isotropic materials and is a continuum theory, while atomic level studies are obviously anisotropic and discrete-particle based. Hence no effort will be made to compare the results to classical results, since such a comparison is neither valid nor warranted.

Finally, the very definition of virial stress as a discrete, atomic force/position based stress leads to important differences in observed results from classical macroscopic results. For example, since the voids are all oriented in the $\mathrm{Z}$ direction, the $\mathrm{ZZ}$ stress increment around the voids is expected to be zero always according to classical macroscopic results (at least for the case of plane stress). However, since the atomic positions alternate in the atomic planes of a crystal lattice, the exclusion of neighbouring atoms most definitely induces $\mathrm{ZZ}$ virial stress increments, even though the crystal is unloaded. Again, one cannot expect atomic results to be similar to classical macroscopic results in all respects.

The author was unable to find any work in the literature archives to compare the results with. Hence the stress increment results are simply presented in this chapter. The study was restricted to perfect crystals with infinite voids since modelling dislocations in crystals using MD is beyond the scope of this thesis.

\subsection{Chapter Summary}

Virial stress increment patterns in deformed copper crystal lattices with voids of various geometries were studied using MD. All six independent components of the incremental stress tensor were presented as contour plots. The plots were analyzed and differences between them and expectations from classical continuum theories were noted and accounted for. Although the study is idealized, the results provide insight into atomiclevel stress patterns in loaded perfect crystals. 


\section{A BRIDGED FINITE ELEMENT-MOLECULAR DYNAMICS METHOD FOR LARGE ATOMIC SYSTEMS}

\subsection{Introduction to Bridged Method}

This chapter presents a bridged method for efficiently analyzing metal single crystals that combines certain key principles of the Finite Element Method (FEM) with Molecular Dynamics (MD). The method is expected to be faster than MD simulations since only a single step is required for the bridged Finite Element-Molecular Dynamics (FE-MD) method in most cases, while a few thousand time steps are typically required for MD simulations (as seen in the applications in Chapter 4). The element discretization scheme introduced for the bridged FE-MD method is a novel scheme and was developed by the author for this thesis. The bridged FE-MD method essentially reformulates the dynamic equations of MD as a static system of equations for static loading. The definition of the static system of equations mirrors that of conventional FEM, i.e. it uses appropriately defined stiffness equations for each atom in the crystal lattice. Since the Embededed Atom Method (EAM) potential for metals is a multi-body potential and has a non-linear potential gradient, the stiffness equations hold approximately for that state of the system for which they were formulated. For large initial displacements, recalculating and resolving the stiffness equations iteratively will lead to a final converged set of atomic displacements.

Since the atoms themselves are discrete entities having discrete energies in the system, they can be used as the kernels of the finite elements in the analysis. The results of this new discretization technique are comparable to results from pure MD simulation, and it is felt by the author that this technique, combined with faster computers, will allow efficient analysis of large-scale atomic systems. 
The following sections present the details of the proposed bridged FE-MD method. A reader who is not familiar with the basic principles of the FEM is urged to refer to the numerous good reference books available on the subject, since these principles are not repeated in this thesis report.

\subsection{General Form of Finite Element Stiffness Equation}

Elements in conventional FEM can seemingly be formulated in a variety of ways, but the fundamental quantity used in all these formulations is the energy of the system. The most general way of formulating the stiffness matrix of an element from the energy of the element is demonstrated with the simple example of a one dimensional spring element, shown in Figure 39.
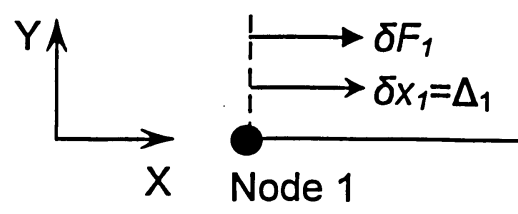

Spring Element with

Energy $U\left(\delta x_{1}, \delta x_{2}\right)$

Node 2

Figure 39 - Two-Node Spring Element (for illustration of general stiffness matrix formulation)

The notations in Figure 39 are self-explanatory. Nodes 1 and 2 experience externally applied forces of magnitudes $\delta F_{1}$ and $\delta F_{2}$ respectively in the $\mathrm{X}$ direction. The resultant displacements of nodes 1 and 2 in the $X$ direction are $\Delta_{1}$ and $\Delta_{2}$ respectively. The potential energy of the spring element is a general function of the nodal displacements, i.e.:

$$
U=U\left(\delta x_{1}, \delta x_{2}\right)
$$


The internal forces on nodes 1 and 2 in any configuration are the negative spatial gradients of the total system potential energy $U$ with respect to the nodal coordinates (or nodal displacements, if the undeformed state is arbitrarily chosen to be at zero potential) in that configuration:

$$
\begin{aligned}
& F_{1, \text { int }}=-\left.\frac{\partial U}{\partial\left(\delta x_{1}\right)}\right|_{\substack{\delta x_{1}=\Delta_{1} \\
\delta x_{2}=\Delta_{2}}} F_{2, \text { int }}=-\left.\frac{\partial U}{\partial\left(\delta x_{2}\right)}\right|_{\substack{\delta x^{2}=\Delta_{1} \\
x_{2}=\Delta_{2}}}
\end{aligned}
$$

The multi-variable first-order Taylor series approximations for the above energy derivatives about the undeformed configuration are:

$$
\left.\left.\frac{\partial U}{\partial\left(\delta x_{1}\right)}\right|_{\substack{\delta x_{1}=\Delta_{1} \\ \delta x_{2}=\Delta_{2}}} \approx \frac{\partial U}{\partial\left(\delta x_{1}\right)}\right|_{\substack{\delta x_{1}=0 \\ \delta x_{2}=0}}+\left.\left(\Delta_{1}\right) \frac{\partial^{2} U}{\partial\left(\delta x_{1}\right)^{2}}\right|_{\substack{\delta x_{1}=0 \\ \delta x_{2}=0}}+\left.\left(\Delta_{2}\right) \frac{\partial^{2} U}{\partial\left(\delta x_{2}\right) \partial\left(\delta x_{1}\right)}\right|_{\substack{\delta_{1}=0 \\ \delta x_{2}=0}}
$$

, and:

$$
\left.\left.\frac{\partial U}{\partial\left(\delta x_{2}\right)}\right|_{\substack{\delta x_{1}=\Delta_{1} \\ \delta x_{2}=\Delta_{2}}} \approx \frac{\partial U}{\partial\left(\delta x_{2}\right)}\right|_{\substack{\delta x_{1}=0 \\ x_{2}=0}}+\left.\left(\Delta_{1}\right) \frac{\partial^{2} U}{\partial\left(\delta x_{1}\right) \partial\left(\delta x_{2}\right)}\right|_{\substack{\delta x_{1}=0 \\ \delta x_{2}=0}}+\left.\left(\Delta_{2}\right) \frac{\partial^{2} U}{\partial\left(\delta x_{2}\right)^{2}}\right|_{\substack{\delta x_{1}=0 \\ \delta x_{2}=0}}
$$

Under equilibrium, the vector sum of the external applied forces and internal forces at each node is zero:

$$
\left\{\begin{array}{l}
F_{1, e x t} \\
F_{2, e x t}
\end{array}\right\}+\left\{\begin{array}{l}
F_{1, \text { int }} \\
F_{2, \text { int }}
\end{array}\right\}=\left\{\begin{array}{l}
0 \\
0
\end{array}\right\}
$$

Combining equations (82) through (86) gives the following final stiffness equation for the non-linear spring element: 


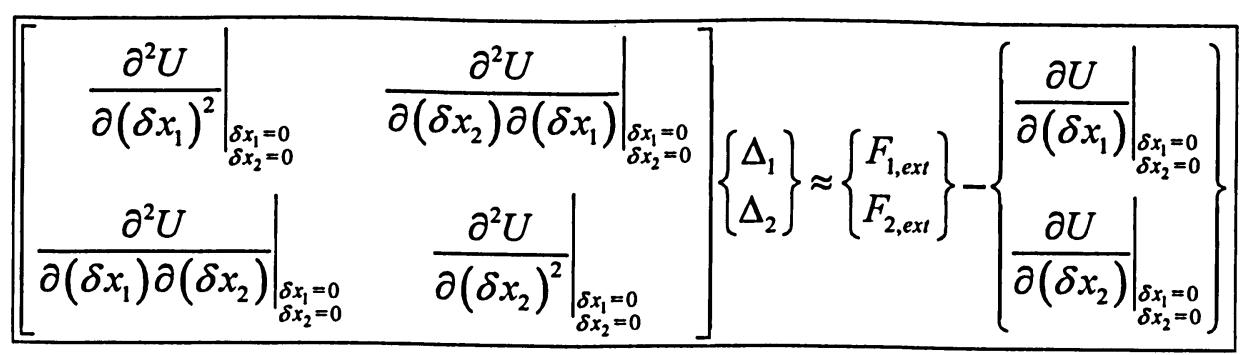

Equation (87) resembles the standard FEM global stiffness equation:

$$
[K]\{d\}=\{F\}
$$

, with the exception of the approximation inherent in the derivation of equation (87). Comparing equation (87) with equation (88), it is apparent that:

$$
\begin{aligned}
& {[K]=\left[\begin{array}{cc}
\left.\frac{\partial^{2} U}{\partial\left(\delta x_{1}\right)^{2}}\right|_{\substack{\delta x_{1}=0 \\
\delta x_{2}=0}} & \left.\frac{\partial^{2} U}{\partial\left(\delta x_{2}\right) \partial\left(\delta x_{1}\right)}\right|_{\substack{\delta x_{1}=0 \\
\delta x_{2}=0}} \\
\left.\frac{\partial^{2} U}{\partial\left(\delta x_{1}\right) \partial\left(\delta x_{2}\right)}\right|_{\substack{\delta x_{1}=0 \\
\delta x_{2}=0}} & \left.\frac{\partial^{2} U}{\partial\left(\delta x_{2}\right)^{2}}\right|_{\substack{\delta x_{1}=0 \\
\delta x_{2}=0}}
\end{array}\right]} \\
& \{d\}=\left\{\begin{array}{l}
\Delta_{1} \\
\Delta_{2}
\end{array}\right\}
\end{aligned}
$$

, and:

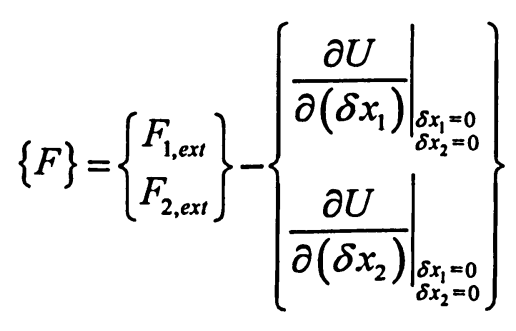


As mentioned earlier, an iterative procedure that involves updating the stiffness equation will ensure convergence to the exact nodal displacements (i.e. will overcome the approximation in equation (87)).

Using a similar approach, the stiffness equation for any non-linear system having Cartesian nodal degrees of freedom $\left(x_{1}, x_{2}, \ldots, x_{n}\right)$ and total energy $U_{t o t}$ is:

$$
\left[\begin{array}{cccc}
\frac{\partial^{2} U_{t o t}}{\partial x_{1}^{2}} & \frac{\partial^{2} U_{t o t}}{\partial x_{2} \partial x_{1}} & \cdots & \frac{\partial^{2} U_{t o t}}{\partial x_{n} \partial x_{1}} \\
\frac{\partial^{2} U_{t o t}}{\partial x_{1} \partial x_{2}} & \frac{\partial^{2} U_{t o t}}{\partial x_{2}^{2}} & \cdots & \cdot \\
\cdot & \cdot & \cdot & \cdot \\
\cdot & \cdot & \cdot & \cdot \\
\cdot & \cdot & \cdot & \cdot \\
\frac{\partial^{2} U_{t o t}}{\partial x_{1} \partial x_{n}} & \cdots & \ldots & \frac{\partial^{2} U_{t o t}}{\partial x_{n}{ }^{2}}
\end{array}\right]\left\{\begin{array}{c}
x_{1} \\
x_{2} \\
\cdot \\
\cdot \\
x_{n}
\end{array}\right\} \approx\left\{\begin{array}{c}
F_{1, e x t} \\
F_{2, e x t} \\
\cdot \\
\cdot \\
\cdot \\
F_{n, e x t}
\end{array}\right\}-\left\{\begin{array}{c}
\frac{\partial U_{t o t}}{\partial x_{1}} \\
\frac{\partial U_{t o t}}{\partial x_{2}} \\
\cdot \\
\cdot \\
\cdot \\
\frac{\partial U_{t o t}}{\partial x_{n}}
\end{array}\right\}
$$

Equation (92) is the most general form of the FE stiffness equation of a system. It should be remembered that since the total energy of the system has to be used in equation (92), the resulting stiffness equation is the stiffness equation for the entire model, and not for individual elements of the model. Individual element stiffness matrices can be formulated similar to equation (92) if and only if the total system energy, $U_{t o t}$, can be distributed between the elements in an independent and mutually exclusive manner. As will be seen shortly, this is possible with the EAM energy if each finite element is based upon one atom of the model and the energy of that atom is taken to be the energy of the corresponding element.

\subsection{Fe Boundary Conditions vs. MD Periodic Boundary Conditions}

In conventional FEM, all boundary conditions are applied to the boundary nodes as the name suggests. However, in MD, there are no boundary nodes for an infinite or semi- 
infinite crystal lattice. To overcome this, MD implements Periodic Boundary Condition (PBC) which simulates the effect of an infinite surrounding crystal lattice on the model being simulated (already described in Section 2.4.3.4).

The importance of including the atoms in the PBC cells in the bridged FE-MD analysis is illustrated very nicely with the following simple example of an assembly of linear spring elements, each with stiffness $k$ :

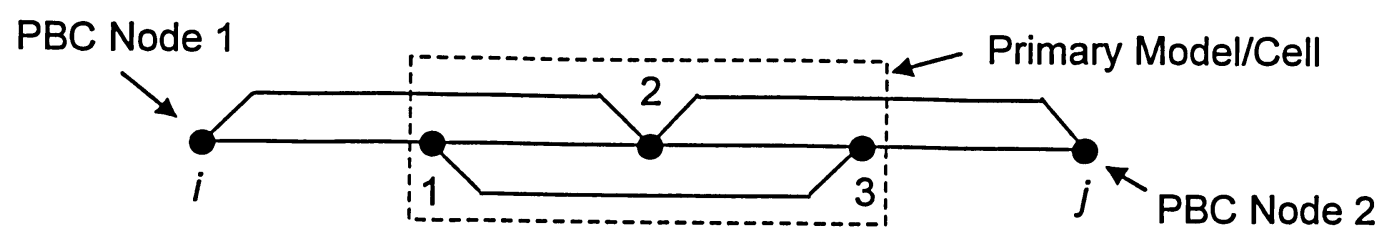

Figure 40 - Assembly of Linear Spring Elements (for illustration of the effects of PBC)

Nodes 1, 2 and 3 comprise the primary cell, while nodes $i$ and $j$ enforce partial PBC on the primary model through their influence on nodes 1,2 and 3.

Using conventional FEM techniques for spring elements, it can be easily calculated that the stiffness matrix equation for the entire model is:

$$
k\left[\begin{array}{ccccc}
2 & -1 & -1 & 0 & 0 \\
-1 & 3 & -1 & -1 & 0 \\
-1 & -1 & 4 & -1 & -1 \\
0 & -1 & -1 & 3 & -1 \\
0 & 0 & -1 & -1 & 2
\end{array}\right]\left\{\begin{array}{l}
x_{i} \\
x_{1} \\
x_{2} \\
x_{3} \\
x_{j}
\end{array}\right\}=\left\{\begin{array}{l}
F_{1} \\
F_{1} \\
F_{2} \\
F_{3} \\
F_{j}
\end{array}\right\}
$$

, where $F_{i}$ denotes the net external force on node $i$. Now, if the model is strained such as to displace node $j$ to the right by an amount $\delta$ while retaining the position of node $i$, then the FE boundary conditions are: 


$$
\left\{\begin{array}{l}
x_{i} \\
x_{j}
\end{array}\right\}=\left\{\begin{array}{l}
0 \\
\delta
\end{array}\right\}
$$

, and:

$$
\left\{\begin{array}{l}
F_{1} \\
F_{2} \\
F_{3}
\end{array}\right\}=\left\{\begin{array}{l}
0 \\
0 \\
0
\end{array}\right\}
$$

Applying these boundary conditions to equation (93) and solving for the unknown displacements yields:

$$
\left\{\begin{array}{l}
x_{1} \\
x_{2} \\
x_{3}
\end{array}\right\}=-\left[\begin{array}{ccc}
3 & -1 & -1 \\
-1 & 4 & -1 \\
-1 & -1 & 3
\end{array}\right]^{-1}\left[\begin{array}{cc}
-1 & 0 \\
-1 & -1 \\
0 & -1
\end{array}\right]\left\{\begin{array}{l}
0 \\
\delta
\end{array}\right\}
$$

For example, if $\delta$ is 1 , then:

$$
\left\{\begin{array}{l}
x_{1} \\
x_{2} \\
x_{3}
\end{array}\right\}=\left\{\begin{array}{l}
0.375 \\
0.500 \\
0.625
\end{array}\right\}
$$

These values make intuitive sense since the symmetry of the entire model is retained, as is expected from the arrangement in Figure 40.

However, if only the nodes in the primary model are used, then the stiffness matrix equation looks as follows:

$$
\left[\begin{array}{ccc}
3 & -1 & -1 \\
-1 & 4 & -1 \\
-1 & -1 & 3
\end{array}\right]\left\{\begin{array}{l}
x_{1} \\
x_{2} \\
x_{3}
\end{array}\right\}=\left\{\begin{array}{l}
F_{1} \\
F_{2} \\
F_{3}
\end{array}\right\}
$$


If boundary conditions $x_{1}=0, x_{3}=1$ and $F_{2}=0$ are applied to equation (98), then the unknown displacement is calculated as $x_{2}=0.25$, which is completely against the symmetry of the model. This simple example shows why the PBC nodes are important and why they cannot be discarded from the bridged FE-MD analysis.

That being said, the analysis is greatly simplified by the fact that the deformation of the PBC cells is identical to the deformation of the primary cell far away from any voids that are introduced in the primary cell. This means that there are definite relationships between the displacements of the atoms in the PBC cells and the displacement of atoms in the primary cell. This fact will be used shortly to provide a tractable method of solving the bridged FE-MD model.

\subsection{Proposed Element Discretization Scheme for Bridged FE-MD Method}

This section presents the element discretization scheme proposed by the author for the bridged FE-MD method. As mentioned in Section 5.2, a stiffness equation similar to equation (92) can be formulated for each element individually if and only if the total system energy can be distributed between the elements in an independent and mutually exclusive manner. The proposed discretization scheme achieves exactly this for the atomic system. The proposed scheme is now demonstrated using a simple example of a collection of nodes (or atoms) interacting via the simple linear spring potential.

\subsubsection{Conventional FEM Element Discretization Scheme}

Consider the following collection of nodes (or atoms), each interacting with two nearest atoms on either side via the linear spring potential. The linear spring potential is a simple 2-body potential (with each body being a node). Hence the most logical way of discretizing the model is to create one element for each non-repeating pair of nodes $i$ and $j$. The resulting element is readily identified as the two node spring element seen in 
conventional FEM, and in many other common mechanical applications. The resulting model is shown in Figure 41, and is the model used in conventional FE analysis.

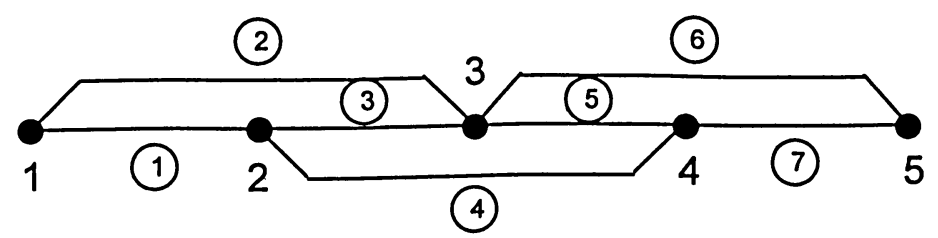

Figure 41 - Assembly of Linear Spring Elements (for illustration of the effects of PBCs)

The potential energy of the spring element containing nodes $i$ and $j$ is then:

$$
U_{i j}=\frac{1}{2} k\left(x_{i}-x_{j}\right)^{2}
$$

, where $k$ is a parameter characterizing the spring potential (commonly called the spring stiffness coefficient, or simply spring stiffness). The element stiffness equation for element 1 is readily determined as:

$$
k\left[\begin{array}{ccccc}
1 & -1 & 0 & 0 & 0 \\
-1 & 1 & 0 & 0 & 0 \\
0 & 0 & 0 & 0 & 0 \\
0 & 0 & 0 & 0 & 0 \\
0 & 0 & 0 & 0 & 0
\end{array}\right]\left\{\begin{array}{l}
x_{1} \\
x_{2} \\
x_{3} \\
x_{4} \\
x_{5}
\end{array}\right\}=\left\{\begin{array}{l}
F_{1}^{1} \\
F_{2}^{1} \\
F_{3}^{1} \\
F_{4}^{1} \\
F_{5}^{1}
\end{array}\right\}
$$

, where $x_{i}$ is the displacement of node (or atom) $i$ and $F_{i}^{j}$ is the force on node $i$ of element $j$. The global stiffness matrix for element 1 is then: 


$$
[K]_{1}=k\left[\begin{array}{ccccc}
1 & -1 & 0 & 0 & 0 \\
-1 & 1 & 0 & 0 & 0 \\
0 & 0 & 0 & 0 & 0 \\
0 & 0 & 0 & 0 & 0 \\
0 & 0 & 0 & 0 & 0
\end{array}\right]
$$

Similarly, the global element stiffness matrices for the other elements are:

$$
\begin{aligned}
& {[K]_{2}=k\left[\begin{array}{ccccc}
1 & 0 & -1 & 0 & 0 \\
0 & 0 & 0 & 0 & 0 \\
-1 & 0 & 1 & 0 & 0 \\
0 & 0 & 0 & 0 & 0 \\
0 & 0 & 0 & 0 & 0
\end{array}\right]} \\
& {[K]_{3}=k\left[\begin{array}{ccccc}
0 & 0 & 0 & 0 & 0 \\
0 & 1 & -1 & 0 & 0 \\
0 & -1 & 1 & 0 & 0 \\
0 & 0 & 0 & 0 & 0 \\
0 & 0 & 0 & 0 & 0
\end{array}\right]} \\
& {[K]_{4}=k\left[\begin{array}{ccccc}
0 & 0 & 0 & 0 & 0 \\
0 & 1 & 0 & -1 & 0 \\
0 & 0 & 0 & 0 & 0 \\
0 & -1 & 0 & 1 & 0 \\
0 & 0 & 0 & 0 & 0
\end{array}\right]} \\
& {[K]_{5}=k\left[\begin{array}{ccccc}
0 & 0 & 0 & 0 & 0 \\
0 & 0 & 0 & 0 & 0 \\
0 & 0 & 1 & -1 & 0 \\
0 & 0 & -1 & 1 & 0 \\
0 & 0 & 0 & 0 & 0
\end{array}\right]}
\end{aligned}
$$




$$
\begin{gathered}
{[K]_{6}=k\left[\begin{array}{ccccc}
0 & 0 & 0 & 0 & 0 \\
0 & 0 & 0 & 0 & 0 \\
0 & 0 & 1 & 0 & -1 \\
0 & 0 & 0 & 0 & 0 \\
0 & 0 & -1 & 0 & 1
\end{array}\right]} \\
{[K]_{7}=k\left[\begin{array}{ccccc}
0 & 0 & 0 & 0 & 0 \\
0 & 0 & 0 & 0 & 0 \\
0 & 0 & 0 & 0 & 0 \\
0 & 0 & 0 & 1 & -1 \\
0 & 0 & 0 & -1 & 1
\end{array}\right]}
\end{gathered}
$$

The assembled global stiffness matrix for the entire model is simply:

$$
[K]_{g}=\sum_{i=1}^{7}[K]_{i}=k\left[\begin{array}{ccccc}
2 & -1 & -1 & 0 & 0 \\
-1 & 3 & -1 & -1 & 0 \\
-1 & -1 & 4 & -1 & -1 \\
0 & -1 & -1 & 3 & -1 \\
0 & 0 & -1 & -1 & 2
\end{array}\right]
$$

The global model stiffness matrix obtained from the conventional FE discretization scheme (equation (108)) will be compared to that obtained from the proposed FE discretization scheme, which is the focus of the next section.

\subsubsection{Proposed Element Discretization Scheme}

Consider the same collection of nodes (or atoms) as in the previous section, each again interacting with two nearest atoms on either side via the linear spring potential. The potential energy of a pair of nodes $i$ and $j$ is then given by the following expression: 


$$
U_{i j}=\frac{1}{2} k\left(x_{i}-x_{j}\right)^{2}
$$

, where $k$ is again the parameter characterizing the linear spring potential. Now, this potential energy can be divided equally between the two nodes, giving the following expression for potential energy per node $i$ or $j$ for an interaction between those nodes:

$$
U_{i}=U_{j}=\frac{1}{4} k\left(x_{i}-x_{j}\right)^{2}
$$

The ability to divide energy between the nodes in this fashion (i.e. in an independent and mutually exclusive manner) suggests the use of a rather different element for the $\mathrm{FE}$ discretization: one in which the element energy is based on the total energy of a single node. Such an element based on node 1, for example, would look as depicted in Figure 42:

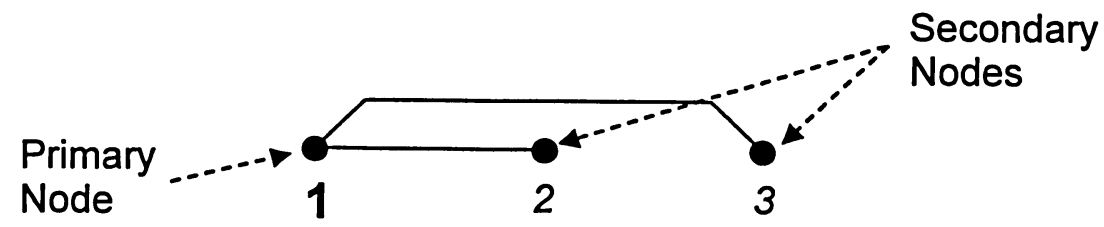

Figure 42 - Proposed Element Discretization (only element 1 shown)

The energy of the element (element 1) shown in Figure 42 is taken to be equal to the total energy of node 1 , which is calculated using equation (110) from all non-repeating interaction pairs that involve node 1 . The element number is equal to the number of the node upon whose energy the element energy is based. This node is termed the primary node of the element. All nodes that interact with the primary node are included in the element and are called secondary nodes. Note that the secondary nodes of an element do not contribute to the element energy: they are included because their position relative to the primary node affects the energy of the primary node, which has to be captured in the 
stiffness matrix. Now, the formulation of the stiffness matrix based on this element discretization scheme is addressed.

Using equation (110), the total energy of element 1 (i.e. the energy associated with node 1) is:

$$
U_{1}=\frac{1}{4} k\left[\left(x_{2}-x_{1}\right)^{2}+\left(x_{3}-x_{1}\right)^{2}\right]
$$

Using a similar element discretization procedure, it can be easily verified that the energies of elements 2 through 5 are:

$$
\begin{gathered}
U_{2}=\frac{1}{4} k\left[\left(x_{2}-x_{1}\right)^{2}+\left(x_{2}-x_{3}\right)^{2}+\left(x_{2}-x_{4}\right)^{2}\right] \\
U_{3}=\frac{1}{4} k\left[\left(x_{3}-x_{1}\right)^{2}+\left(x_{3}-x_{2}\right)^{2}+\left(x_{3}-x_{4}\right)^{2}+\left(x_{3}-x_{5}\right)^{2}\right] \\
U_{4}=\frac{1}{4} k\left[\left(x_{4}-x_{2}\right)^{2}+\left(x_{4}-x_{3}\right)^{2}+\left(x_{4}-x_{5}\right)^{2}\right] \\
U_{5}=\frac{1}{4} k\left[\left(x_{5}-x_{3}\right)^{2}+\left(x_{5}-x_{4}\right)^{2}\right]
\end{gathered}
$$

Since the energies of the elements are independent and mutually exclusive of each other, equation (92) can be modified for each element by using the element energy in place of the total system energy. Hence the stiffness matrix for element $i$ is: 


$$
[K]_{i}=\left[\begin{array}{cccc}
\frac{\partial^{2} U_{i}}{\partial x_{1}^{2}} & \frac{\partial^{2} U_{i}}{\partial x_{2} \partial x_{1}} & \cdots & \frac{\partial^{2} U_{i}}{\partial x_{n} \partial x_{1}} \\
\frac{\partial^{2} U_{i}}{\partial x_{1} \partial x_{2}} & \frac{\partial^{2} U_{i}}{\partial x_{2}{ }^{2}} & \cdots & \cdot \\
\cdot & \cdot & \cdot & \cdot \\
\frac{\partial^{2} U_{i}}{\partial x_{1} \partial x_{n}} & \cdots & \cdots & \frac{\partial^{2} U_{i}}{\partial x_{n}^{2}}
\end{array}\right]
$$

, where $U_{i}$ is the energy of element $i$ and $x_{i}$ is the displacement of node $i$. Using equation (116), the global stiffness matrix for element 1 is:

$$
[K]_{1}=\left[\begin{array}{lllll}
\frac{\partial^{2} U_{1}}{\partial x_{1}^{2}} & \frac{\partial^{2} U_{1}}{\partial x_{2} \partial x_{1}} & \frac{\partial^{2} U_{1}}{\partial x_{3} \partial x_{1}} & \frac{\partial^{2} U_{1}}{\partial x_{4} \partial x_{1}} & \frac{\partial^{2} U_{1}}{\partial x_{5} \partial x_{1}} \\
\frac{\partial^{2} U_{1}}{\partial x_{1} \partial x_{2}} & \frac{\partial^{2} U_{1}}{\partial x_{2}^{2}} & \frac{\partial^{2} U_{1}}{\partial x_{3} \partial x_{2}} & \frac{\partial^{2} U_{1}}{\partial x_{4} \partial x_{2}} & \frac{\partial^{2} U_{1}}{\partial x_{5} \partial x_{2}} \\
\frac{\partial^{2} U_{1}}{\partial x_{1} \partial x_{3}} & \frac{\partial^{2} U_{1}}{\partial x_{2} \partial x_{3}} & \frac{\partial^{2} U_{1}}{\partial x_{3}^{2}} & \frac{\partial^{2} U_{1}}{\partial x_{4} \partial x_{3}} & \frac{\partial^{2} U_{1}}{\partial x_{5} \partial x_{3}} \\
\frac{\partial^{2} U_{1}}{\partial x_{1} \partial x_{4}} & \frac{\partial^{2} U_{1}}{\partial x_{2} \partial x_{4}} & \frac{\partial^{2} U_{1}}{\partial x_{3} \partial x_{4}} & \frac{\partial^{2} U_{1}}{\partial x_{4}{ }^{2}} & \frac{\partial^{2} U_{1}}{\partial x_{5} \partial x_{4}} \\
\frac{\partial^{2} U_{1}}{\partial x_{1} \partial x_{5}} & \frac{\partial^{2} U_{1}}{\partial x_{2} \partial x_{5}} & \frac{\partial^{2} U_{1}}{\partial x_{3} \partial x_{5}} & \frac{\partial^{2} U_{1}}{\partial x_{4} \partial x_{5}} & \frac{\partial^{2} U_{1}}{\partial x_{5}^{2}}
\end{array}\right]
$$

, which can be easily evaluated using the energy of element 1 (equation (111)) as:

$$
[K]_{1}=\frac{k}{2}\left[\begin{array}{ccccc}
2 & -1 & -1 & 0 & 0 \\
-1 & 1 & 0 & 0 & 0 \\
-1 & 0 & 1 & 0 & 0 \\
0 & 0 & 0 & 0 & 0 \\
0 & 0 & 0 & 0 & 0
\end{array}\right]
$$

Similary, the global stiffness matrices for the other elements in the proposed discretization scheme are: 


$$
\begin{aligned}
{[K]_{2} } & =\frac{k}{2}\left[\begin{array}{ccccc}
1 & -1 & 0 & 0 & 0 \\
-1 & 3 & -1 & -1 & 0 \\
0 & -1 & 1 & 0 & 0 \\
0 & -1 & 0 & 1 & 0 \\
0 & 0 & 0 & 0 & 0
\end{array}\right] \\
{[K]_{3} } & =\frac{k}{2}\left[\begin{array}{ccccc}
1 & 0 & -1 & 0 & 0 \\
0 & 1 & -1 & 0 & 0 \\
-1 & -1 & 4 & -1 & -1 \\
0 & 0 & -1 & 1 & 0 \\
0 & 0 & -1 & 0 & 1
\end{array}\right] \\
{[K]_{4} } & =\frac{k}{2}\left[\begin{array}{ccccc}
0 & 0 & 0 & 0 & 0 \\
0 & 1 & 0 & -1 & 0 \\
0 & 0 & 1 & -1 & 0 \\
0 & -1 & -1 & 3 & -1 \\
0 & 0 & 0 & -1 & 1
\end{array}\right] \\
{[K]_{5}=} & \frac{k}{2}\left[\begin{array}{ccccc}
0 & 0 & 0 & 0 & 0 \\
0 & 0 & 0 & 0 & 0 \\
0 & 0 & 1 & 0 & -1 \\
0 & 0 & 0 & 1 & -1 \\
0 & 0 & -1 & -1 & 2
\end{array}\right]
\end{aligned}
$$

The assembled global model stiffness matrix is then simply:

$$
[K]_{g}=\sum_{i=1}^{5}[K]_{i}=k\left[\begin{array}{ccccc}
2 & -1 & -1 & 0 & 0 \\
-1 & 3 & -1 & -1 & 0 \\
-1 & -1 & 4 & -1 & -1 \\
0 & -1 & -1 & 3 & -1 \\
0 & 0 & -1 & -1 & 2
\end{array}\right]
$$




\subsubsection{Comparison of Discretization Schemes}

There are only 5 elements in the proposed discretization scheme, compared to 7 elements in the conventional discretization scheme (as evident in equation (108)). Now, the global element stiffness matrices obtained from the proposed discretization method (equations (118) through (122)) are not equal to those obtained from the conventional FEM discretization procedure (equations (101) through (107)), and neither should they be expected to be since the discretization procedures are completely different. However, and most significantly, the global model stiffness matrix obtained from the proposed discretization method (equation (123)) is identical to that obtained from the conventional FEM discretization procedure (equation (108)). This makes intuitive sense since the global model stiffness matrix captures the behaviour of the entire system, and should be the same irrespective of the discretization procedure used since the system can only behave in a single way.

The equality of the global model stiffness matrices given by the conventional FE discretization procedure and the proposed discretization procedure has been established. It might appear that the extra complications involved with the proposed element discretization scheme are unwarranted. After all, an element in the proposed scheme (i.e. the element in Figure 42) is just a combination of elements in the conventional discretization scheme (i.e. elements in Figure 41). However, upon a little thought, it becomes apparent that this behaviour is due to the fact that the linear spring potential is a simple 2-body potential. The real utility of the proposed discretization scheme is realized while dealing with complex multi-body potentials like the EAM potential. For the EAM potential, the embedding function embodies the multi-body component of the potential. As seen from equation (4), the embedding function is determined by the total electron density at the location of an atom, and is not distributive in nature (i.e. the embedding function for the sum of two electron densities is not equal to the sum of the embedding functions for each of those two electron densities). It is easily realized that the behaviour of such multi-body potentials can only be captured using the proposed element discretization scheme. 


\subsection{The Bridged FE-MD Method}

\subsubsection{Formulation of Stiffness Equations}

For the FE-MD bridged method, the nodes in Figure 42 can be considered as atoms, with each element consisting of a primary atom interacting with a number of secondary atoms within its sphere of influence. The interactions are, of course, represented by the EAM potential and not by the simple linear spring potential used for the example in Section 5.4. The EAM potential energy of an atom $i$ interacting with $N-1$ atoms within its sphere of influence (which is determined by the EAM potential cut-off distance) is given by the expression within the square brackets in equation (4), i.e.:

$$
U_{i, E A M}=\frac{1}{2} \sum_{\substack{j=1 \\ j \neq i}}^{N} \phi\left(r_{i j}\right)+\Psi\left\{\sum_{\substack{j=1 \\ j \neq i}}^{N} \rho\left(r_{i j}\right)\right\}
$$

The second derivative of the EAM potential energy for atom $i$ with respect to degrees of freedom (nodal displacements) $p$ and $q$ can then be derived easily as:

$$
\begin{aligned}
& \frac{\partial^{2} U_{i}}{\partial q \partial p}=\sum_{\substack{j=1 \\
j \neq i}}^{N}\left[\left\{\frac{1}{2} \frac{d \phi}{d r_{i j}}+\frac{d \Psi}{d \rho_{t o t, i}} \frac{d \rho_{i j}}{d r_{i j}}\right\} \frac{\partial^{2} r_{i j}}{\partial q \partial p}+\left\{\frac{1}{2} \frac{d^{2} \phi}{d r_{i j}^{2}}+\frac{d \Psi}{d \rho_{t o t, i}} \frac{d^{2} \rho_{i j}}{d r_{i j}^{2}}\right\} \frac{\partial r_{i j}}{\partial q} \frac{\partial r_{i j}}{\partial p}\right]+\ldots \\
& \ldots+\frac{d^{2} \Psi}{d \rho_{t o t, i}{ }^{2}} \sum_{\substack{j=1 \\
j \neq i}}^{N}\left[\frac{d \rho_{i j}}{d r_{i j}} \frac{\partial r_{i j}}{\partial q}\right] \sum_{\substack{j=1 \\
j \neq i}}^{N}\left[\frac{d \rho_{i j}}{d r_{i j}} \frac{\partial r_{i j}}{\partial p}\right]
\end{aligned}
$$

In equation (125), $r_{i j}$ is the distance between atoms $i$ and $j$ and, for Cartesian coordinates, is given by: 


$$
r_{i j}=\sqrt{\left(x_{i}-x_{j}\right)^{2}+\left(y_{i}-y_{j}\right)^{2}+\left(z_{i}-z_{j}\right)^{2}}
$$

It can be easily verified that the first derivatives of $r_{i j}$ are then:

$$
\begin{aligned}
& \frac{\partial r_{i j}}{\partial x_{i}}=-\frac{\partial r_{i j}}{\partial x_{j}}=\frac{x_{i}-x_{j}}{r_{i j}} \\
& \frac{\partial r_{i j}}{\partial y_{i}}=-\frac{\partial r_{i j}}{\partial y_{j}}=\frac{y_{i}-y_{j}}{r_{i j}} \\
& \frac{\partial r_{i j}}{\partial z_{i}}=-\frac{\partial r_{i j}}{\partial z_{j}}=\frac{z_{i}-z_{j}}{r_{i j}}
\end{aligned}
$$

, while the second derivatives are:

$$
\begin{aligned}
& \frac{\partial^{2} r_{i j}}{\partial x_{i}{ }^{2}}=-\frac{\partial^{2} r_{i j}}{\partial x_{j} \partial x_{i}}=-\frac{\partial^{2} r_{i j}}{\partial x_{i} \partial x_{j}}=\frac{\partial^{2} r_{i j}}{\partial x_{j}{ }^{2}}=\frac{r_{i j}^{2}-\left(x_{i}-x_{j}\right)^{2}}{r_{i j}^{3}} \\
& \frac{\partial^{2} r_{i j}}{\partial y_{i}{ }^{2}}=-\frac{\partial^{2} r_{i j}}{\partial y_{j} \partial y_{i}}=-\frac{\partial^{2} r_{i j}}{\partial y_{i} \partial y_{j}}=\frac{\partial^{2} r_{i j}}{\partial y_{j}{ }^{2}}=\frac{r_{i j}{ }^{2}-\left(y_{i}-y_{j}\right)^{2}}{r_{i j}{ }^{3}} \\
& \frac{\partial^{2} r_{i j}}{\partial z_{i}{ }^{2}}=-\frac{\partial^{2} r_{i j}}{\partial z_{j} \partial z_{i}}=-\frac{\partial^{2} r_{i j}}{\partial z_{i} \partial z_{j}}=\frac{\partial^{2} r_{i j}}{\partial z_{j}{ }^{2}}=\frac{r_{i j}{ }^{2}-\left(z_{i}-z_{j}\right)^{2}}{r_{i j}^{3}} \\
& \frac{\partial^{2} r_{i j}}{\partial y_{i} \partial x_{i}}=-\frac{\partial^{2} r_{i j}}{\partial y_{j} \partial x_{i}}=-\frac{\partial^{2} r_{i j}}{\partial y_{i} \partial x_{j}}=\frac{\partial^{2} r_{i j}}{\partial y_{j} \partial x_{j}}=\frac{-\left(x_{i}-x_{j}\right)\left(y_{i}-y_{j}\right)}{r_{i j}^{3}} \\
& \frac{\partial^{2} r_{i j}}{\partial z_{i} \partial x_{i}}=-\frac{\partial^{2} r_{i j}}{\partial z_{j} \partial x_{i}}=-\frac{\partial^{2} r_{i j}}{\partial z_{i} \partial x_{j}}=\frac{\partial^{2} r_{i j}}{\partial z_{j} \partial x_{j}}=\frac{-\left(x_{i}-x_{j}\right)\left(z_{i}-z_{j}\right)}{r_{i j}^{3}} \\
& \frac{\partial^{2} r_{i j}}{\partial z_{i} \partial y_{i}}=-\frac{\partial^{2} r_{i j}}{\partial z_{j} \partial y_{i}}=-\frac{\partial^{2} r_{i j}}{\partial z_{i} \partial y_{j}}=\frac{\partial^{2} r_{i j}}{\partial z_{j} \partial y_{j}}=\frac{-\left(y_{i}-y_{j}\right)\left(z_{i}-z_{j}\right)}{r_{i j}^{3}}
\end{aligned}
$$

Using equations (125) through (128) and the element discretization method proposed in Section 5.4.2, the global model stiffness matrix for a system of atoms interacting through the EAM potential can easily be determined. From the standpoint of computational 
implementation, this is accomplished through a single step MD simulation of all the atoms, with extra equations for calculating second derivatives of energy as per equations (125) through (128). This is why the technique is termed a bridged FE-MD method. All that remains is to calculate the forces $F$ on the atoms for use in the following stiffness equation:

$$
[K]_{g}\{d\}=\{F\}
$$

The forces are given by the components of the EAM force vector given in equation (5), and whose general expression is:

$$
F_{p_{i}}=-\frac{\partial U_{t o t, E A M}}{\partial p_{i}}=-\sum_{\substack{j=1 \\ j \neq i}}^{N}\left[\frac{d \phi\left(r_{i j}\right)}{d r_{i j}}+\left(\frac{d \Psi}{d \rho_{i, t o t}}+\frac{d \Psi}{d \rho_{j, t o t}}\right)\left(\frac{d \rho\left(r_{i j}\right)}{d r_{i j}}\right)\right] \frac{\partial r_{i j}}{\partial p_{i}}
$$

Equation (130) gives the component of force on atom $i$ in the direction of degree-offreedom $p_{i}$.

All quantities in equation (129) are known except for the unknown displacements. However, as mentioned in Section 5.3, equation (129) necessarily contains displacements of elements whose primary nodes are the atoms of the PBC cells. But these displacements are related to the displacements of the atoms in the primary cell in a definite manner, since the deformation of the PBC cells is the same as the deformation of the primary cell away from any voids in the primary cell (this was also mentioned in Section 5.3). This will be addressed next.

\subsubsection{Mapping PBC Nodal Displacements to Primary Cell Nodal Displacements}

The code used for performing the bridged FE-MD analysis is a variant of MD_V_7_00. Hence it provides the option of specifying initial strains on the entire model. Since most 
PBC cells are based on the primary cell excluding any voids (except in directions of infinite voids), it seems logical to strain the entire model to the desired strain before running the bridged FE-MD analysis. If this is done, the atoms of the $\mathrm{PBC}$ cells are already in the desired locations and their subsequent displacements (i.e. displacements from the bridged FE-MD analysis) can be taken as zero. Equation (129) can be simplified using this fact since columns of the global stiffness matrix corresponding to the displacements of these atoms are multiplied by zero, resulting in their removal from the analysis.

Further reduction can be achieved by noting that all PBC cells in the direction of infinite voids are exact copies of the primary cell with the void included. Hence the displacements of corresponding atoms between the PBC cells in the direction of infinite voids and the primary cell can be equated to each other. These equalities can be expressed as a matrix equation:

$$
\{d\}=[T]\{d\}_{p}
$$

, where: $\{d\}$ is the vector of nodal displacements of the entire model (including the PBC cells); $\{d\}_{p}$ is the vector of nodal displacements of the primary cell alone; and $[T]$ is the mapping matrix consisting of 0 and 1 , which relates the two displacement vectors with each other. Equation (131) is then substituted into the LHS of equation (129) to reduce it to a system of equations containing only the nodal displacements of the primary cell.

Because the method involves pre-straining the crystal, the bridged FE-MD method can only be used for crystal relaxation studies around cracks or voids. That being said, straining the entire crystal lattice beforehand is a trivial task and therefore the proposed method can be used without any concerns. 


\subsubsection{Boundary Conditions}

Once the mapping procedure of Section 5.5.2 has been implemented, what is left is a stiffness equation that involves only the displacements of atoms in the primary cell. Since the strains have already been applied, the boundary conditions are simple: the atoms on the faces of the primary cell which are connected to neighbouring PBC cells have no displacements, while the forces on the remaining atoms are given by equation (130). Now the system of equations can be solved for the displacements of the primary cell atoms, $\{d\}_{p}$, from which the displacements of the $\mathrm{PBC}$ atoms can be determined using equation (131).

\subsection{Code for Bridged FE-MD Method}

The code for performing the bridged FE-MD analysis is a variant of MD_V_7_00, which has been named FE_MD_V_7_00. The only major difference between MD_V_7_00 and FE_MD_V_7_00 is the presence of code blocks related to the bridged FE-MD method in FE_MD_V_7_00.

\subsection{Chapter Summary}

This chapter presented a bridged FE-MD method for analyzing large atomic systems. Because of the FE based formulation, the bridged method is expected to be much quicker than MD simulation alone. Details of the stiffness matrix formulation for the bridged method were presented, along with all relevant theory. The bridged FE-MD method is implemented in MATLAB ${ }^{\mathrm{TM}}$ code FE_MD_V_7_00 written by the author for this thesis. The next chapter uses FE_MD_V_7_00 to analyze the three cases that were analyzed using MD simulation alone in Chapter 4. The superior efficiency of the bridged FE-MD method will become apparent in the process. 


\section{APPLICATION OF BRIDGED FE-MD METHOD}

\subsection{Overview of Applications}

The bridged FE-MD method will be applied to the analysis of the three cases that have already been simulated by $\mathrm{MD}$ alone in Chapter 4 using FE_MD_V_7_00. Only the stress increment plots are shown, since the bridged FE-MD method has already been outlined in sufficient detail in Chapter 5. Details of the MD simulation and crystal void geometry can be found in Chapter 4.

\subsection{Application 1: Elastically Stressed Infinite, Perfectly Cylindrical Void in a Perfect Copper Crystal Lattice}

The results of the bridged FE-MD analysis using FE_MD_V_7_00 for this case are shown in Figure 43 through Figure 48. 


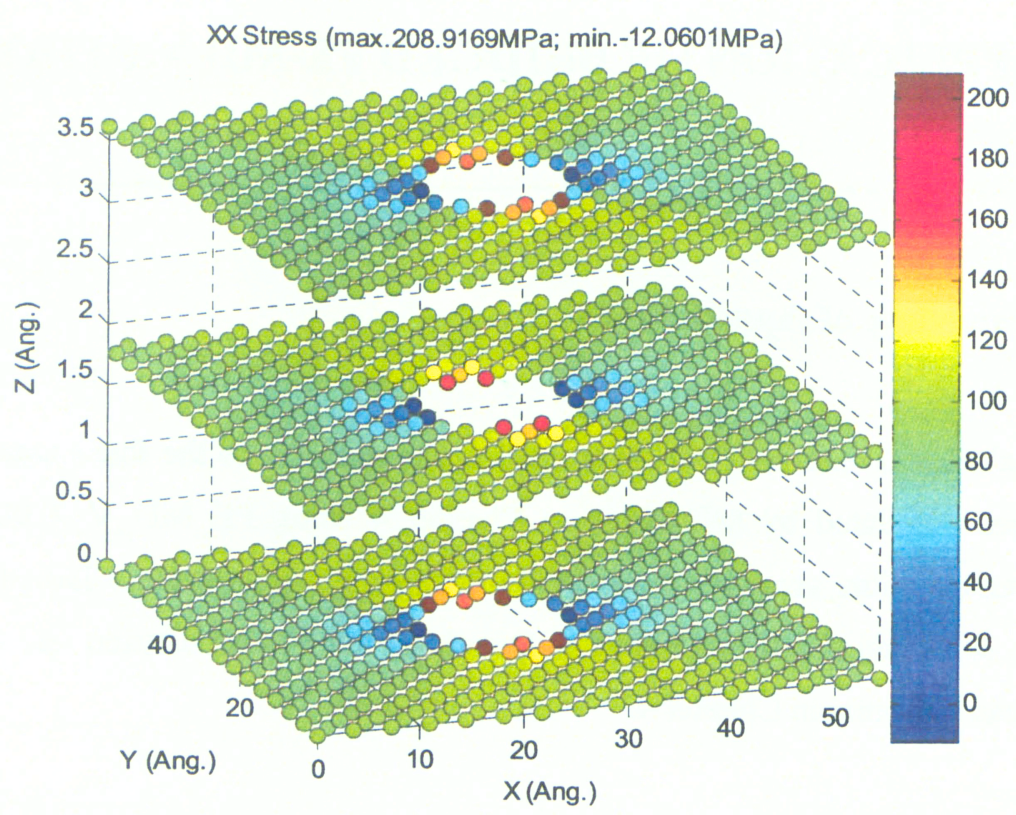

Figure 43 - Application 1: XX Stress Increment from FE-MD Bridged Simulation (MPa)

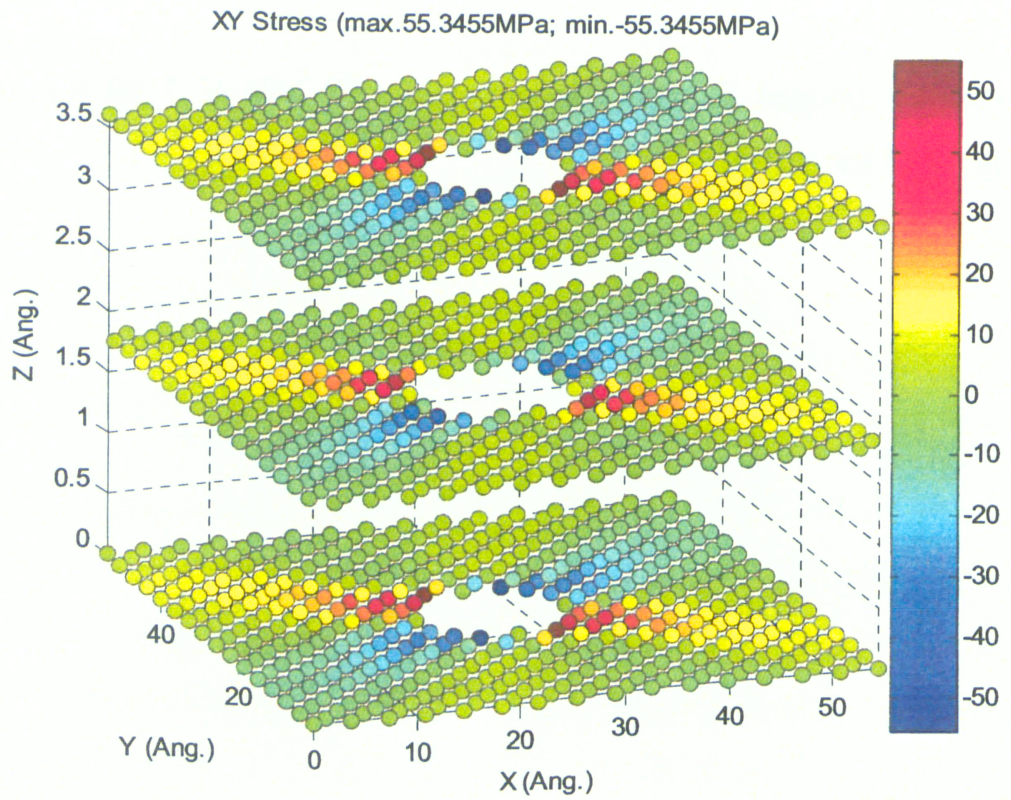

Figure 44 - Application 1: XY (YX) Stress Increment from FE-MD Bridged Simulation (MPa) 


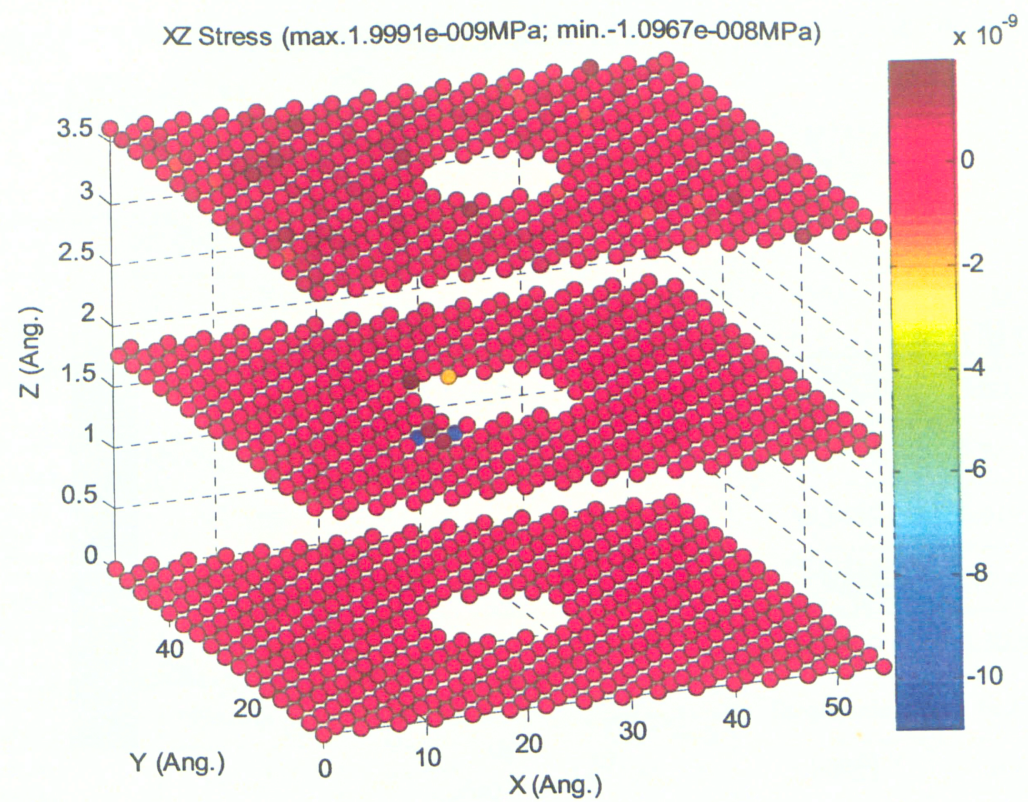

Figure 45 - Application 1: XZ (ZX) Stress Increment from FE-MD Bridged Simulation (MPa)

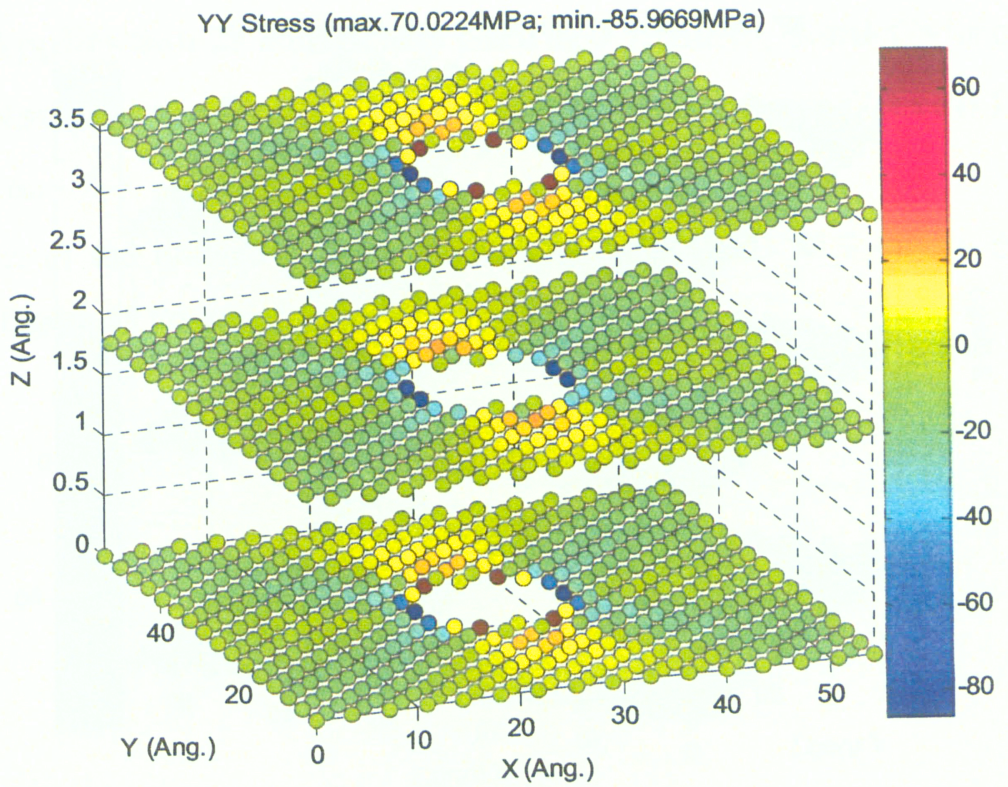

Figure 46 - Application 1: YY Stress Increment from FE-MD Bridged Simulation (MPa) 


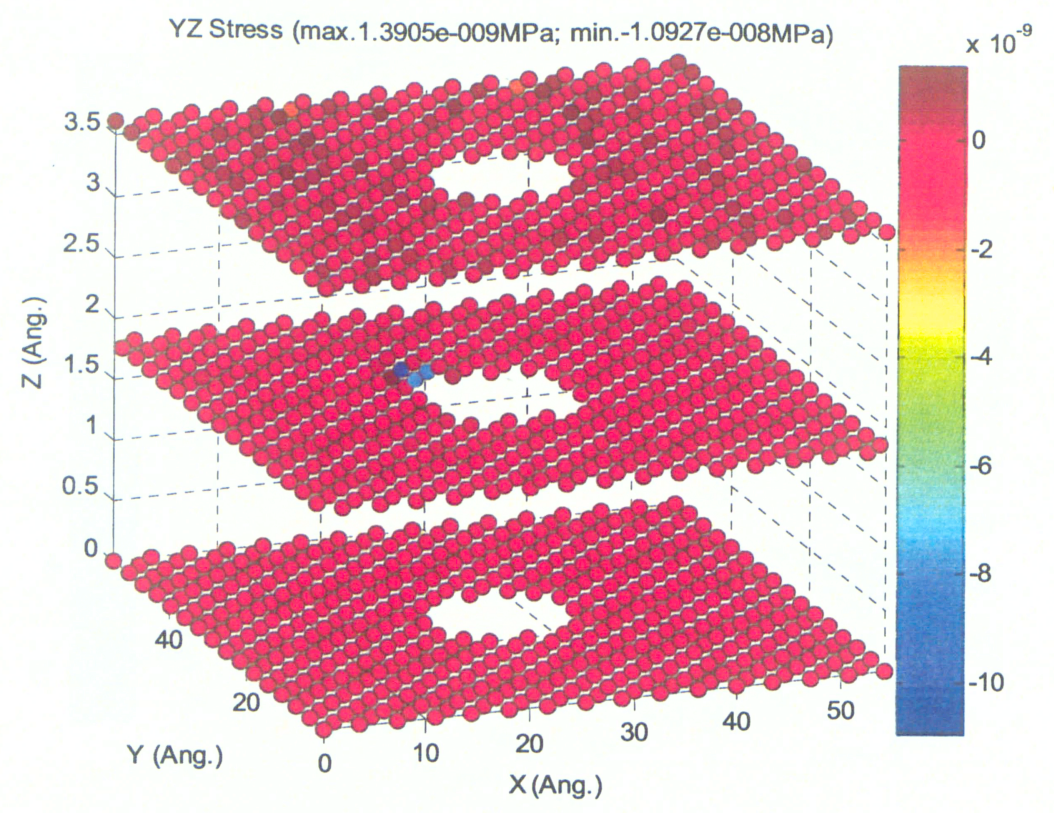

Figure 47 - Application 1: YZ (ZY) Stress Increment from FE-MD Bridged Simulation (MPa)

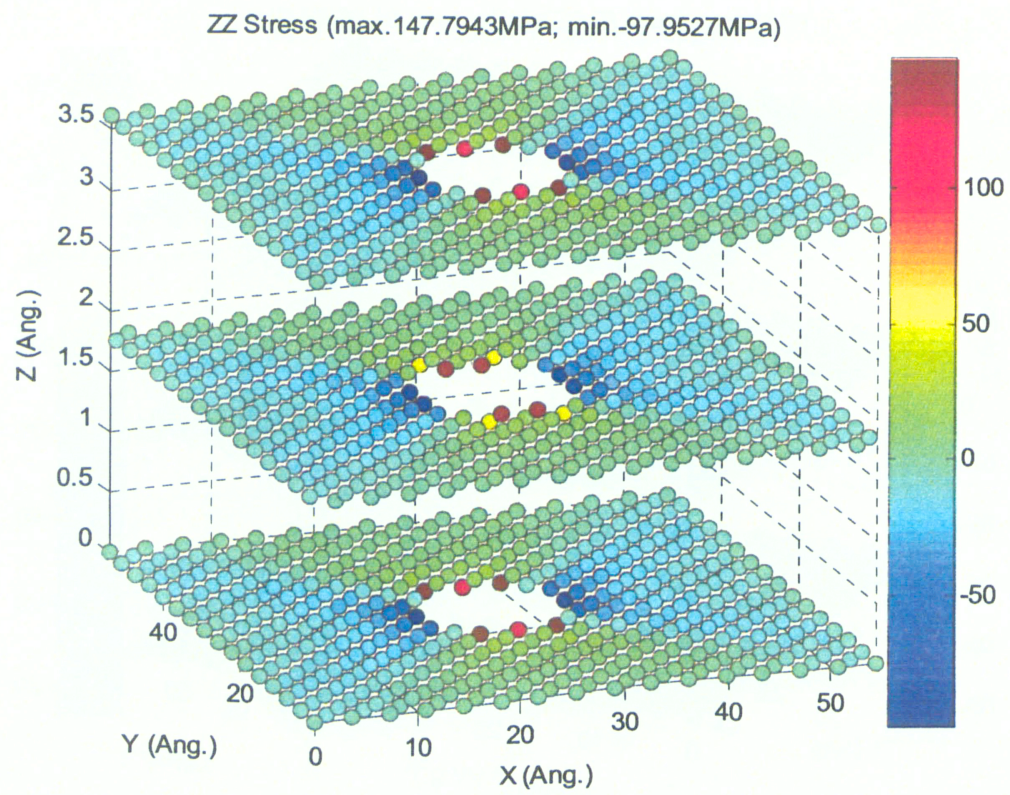

Figure 48 - Application 1: ZZ Stress Increment from FE-MD Bridged Simulation (MPa) 
Table 4 compares the results from the bridged FE-MD method to those from the pure $M D$ simulation (Section 4.2.4).

Table 4-Summary and Comparison of Results for Application 1

\begin{tabular}{|l|c|c|c|c|c|c|}
\hline \multirow{2}{*}{$\begin{array}{c}\text { Quantity of } \\
\text { Interest }\end{array}$} & \multicolumn{2}{c|}{$\begin{array}{c}\text { Pure MD } \\
\text { Simulation }\end{array}$} & \multicolumn{2}{c|}{$\begin{array}{c}\text { Bridged FE-MD } \\
\text { Method }\end{array}$} & \multicolumn{2}{c|}{$\begin{array}{c}\text { Difference } \\
\text { (MPa, not \%) }\end{array}$} \\
\cline { 2 - 7 } & Max. & Min. & Max. & Min. & Max. & Min. \\
\hline $\begin{array}{l}\text { XX Virial Stress } \\
\text { Increment (MPa) }\end{array}$ & 213.8394 & -8.0993 & 208.9169 & -12.0601 & 4.9225 & 3.9608 \\
\hline $\begin{array}{l}\text { XY Virial Stress } \\
\text { Increment (MPa) }\end{array}$ & 55.3260 & -55.3260 & 55.3455 & -55.3455 & 0.0195 & 0.0195 \\
\hline $\begin{array}{l}\text { XZ Virial Stress } \\
\text { Increment (MPa) }\end{array}$ & Negligible & Negligible & Negligible & Negligible & N/A & N/A \\
\hline $\begin{array}{l}\text { YY Virial Stress } \\
\text { Increment (MPa) }\end{array}$ & 67.5198 & -85.4373 & 70.0224 & -85.9669 & 2.5026 & 0.5296 \\
\hline $\begin{array}{l}\text { YZ Virial Stress } \\
\text { Increment (MPa) }\end{array}$ & Negligible & Negligible & Negligible & Negligible & N/A & N/A \\
\hline $\begin{array}{l}\text { ZZ Virial Stress } \\
\text { Increment (MPa) }\end{array}$ & 144.8361 & -95.7719 & 147.7943 & -97.9527 & 2.9582 & 2.1808 \\
\hline Simulation Time & \multicolumn{2}{|c|}{2 hours } & \multicolumn{2}{c|}{15 minutes } & \multicolumn{2}{c|}{ N/A } \\
\hline
\end{tabular}

\subsection{Application 2: Elastically Stressed Infinite, Semi-Cylindrical Notch at the Free Surface of a Perfect Copper Crystal Lattice}

The results of the bridged FE-MD analysis using FE_MD_V_7_00 for this case are shown in Figure 49 through Figure 54. 


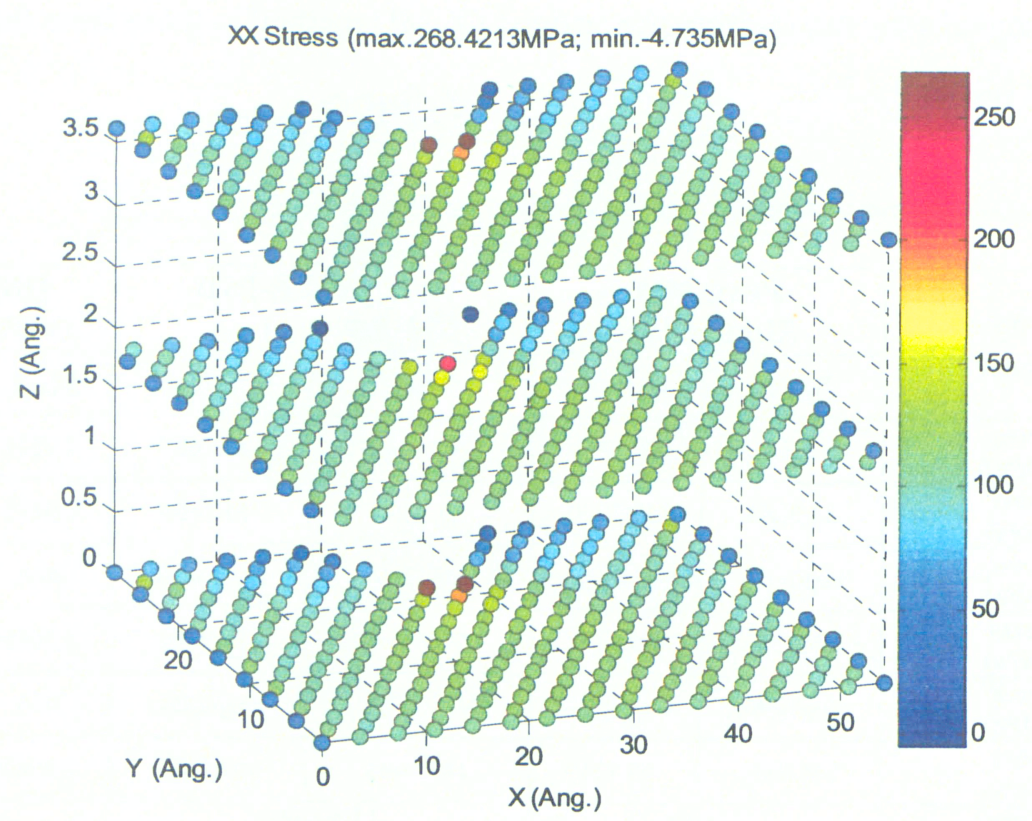

Figure 49 - Application 2: XX Stress Increment from FE-MD Bridged Simulation (MPa)

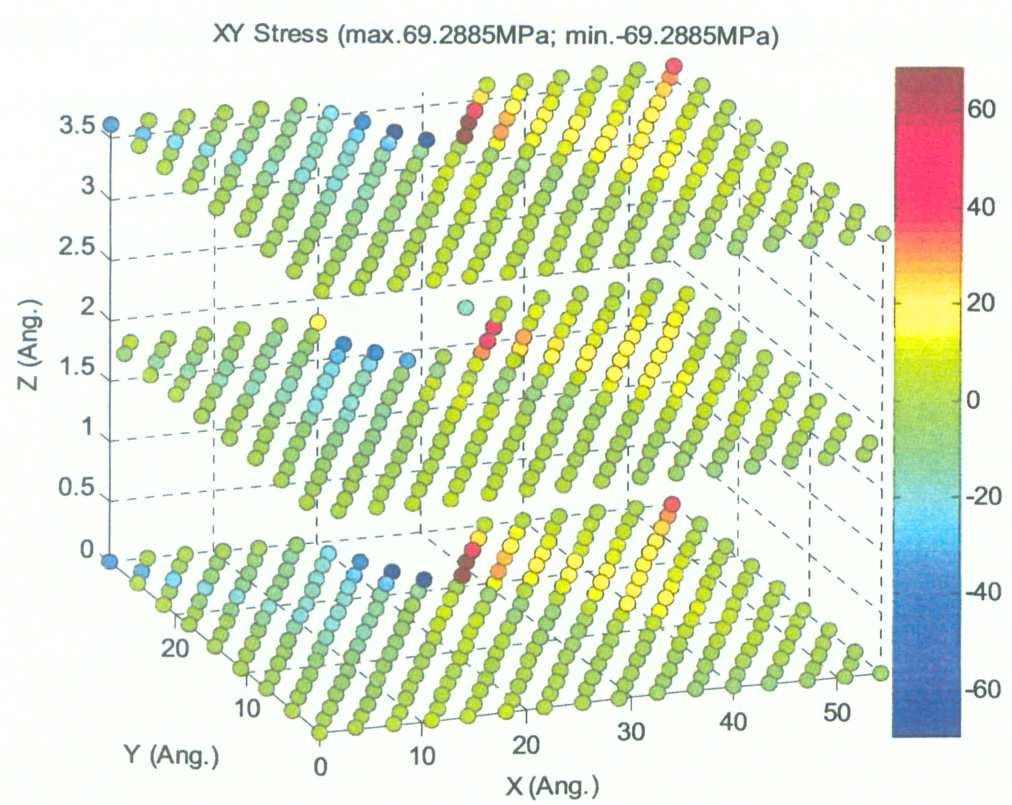

Figure 50 - Application 2: XY (YX) Stress Increment from FE-MD Bridged Simulation (MPa) 


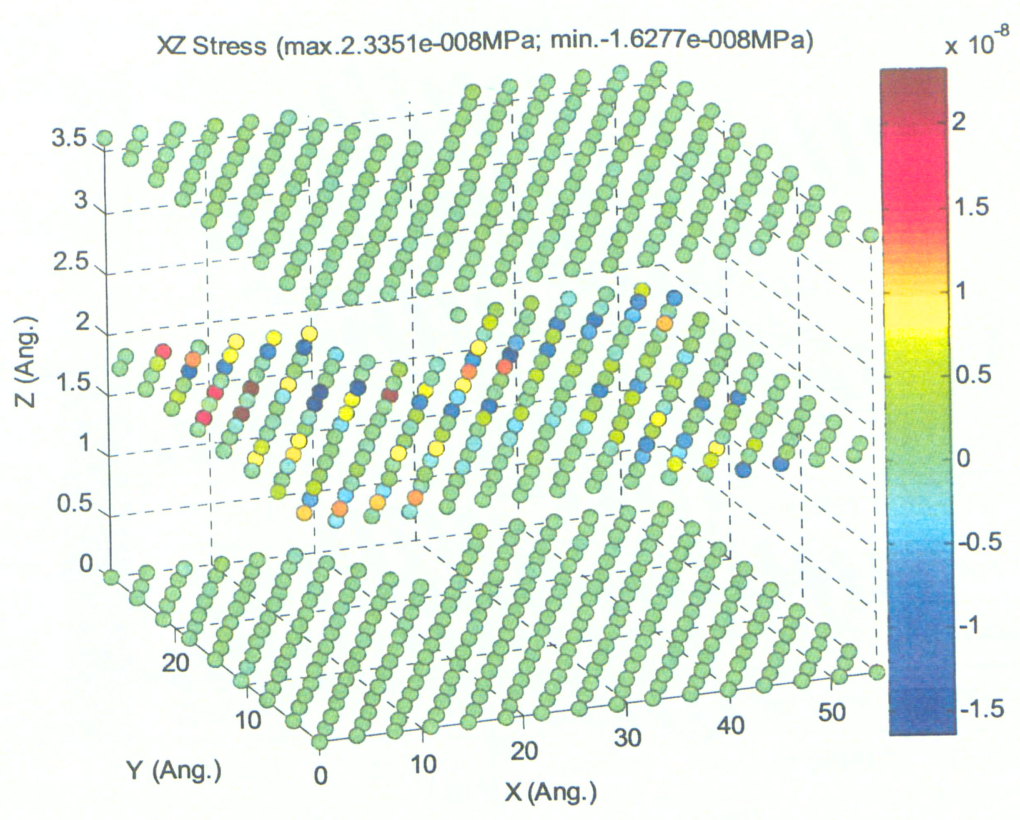

Figure 51 - Application 2: XZ (ZX) Stress Increment from FE-MD Bridged Simulation (MPa)

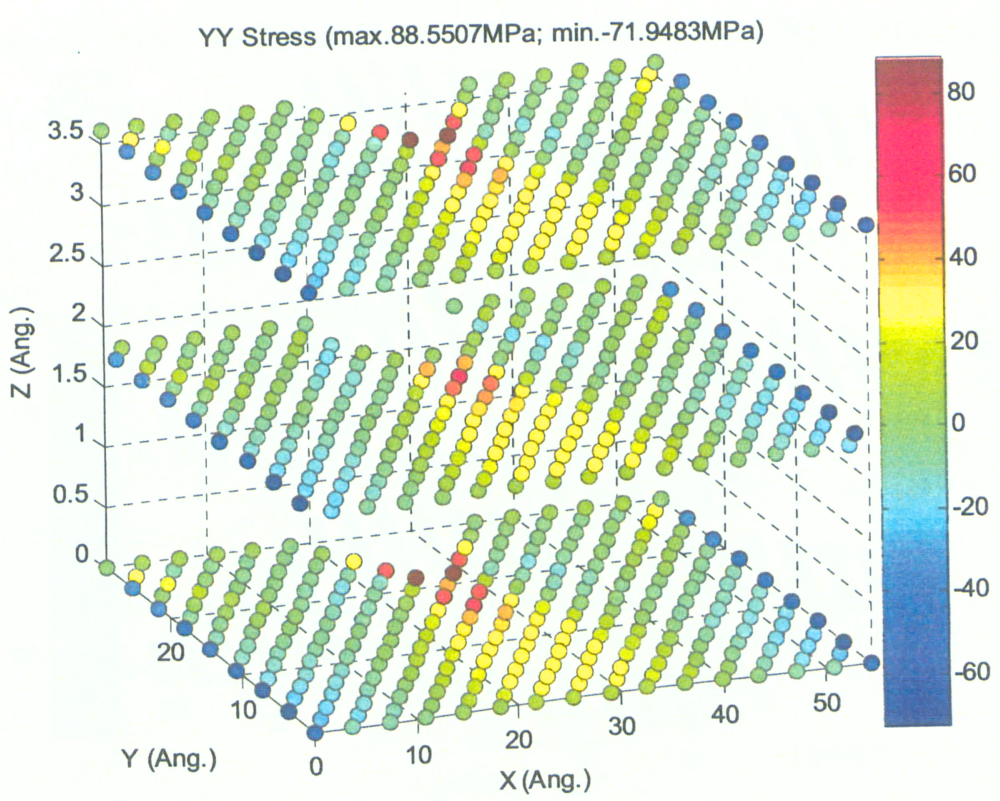

Figure 52 - Application 2: YY Stress Increment from FE-MD Bridged Simulation (MPa) 


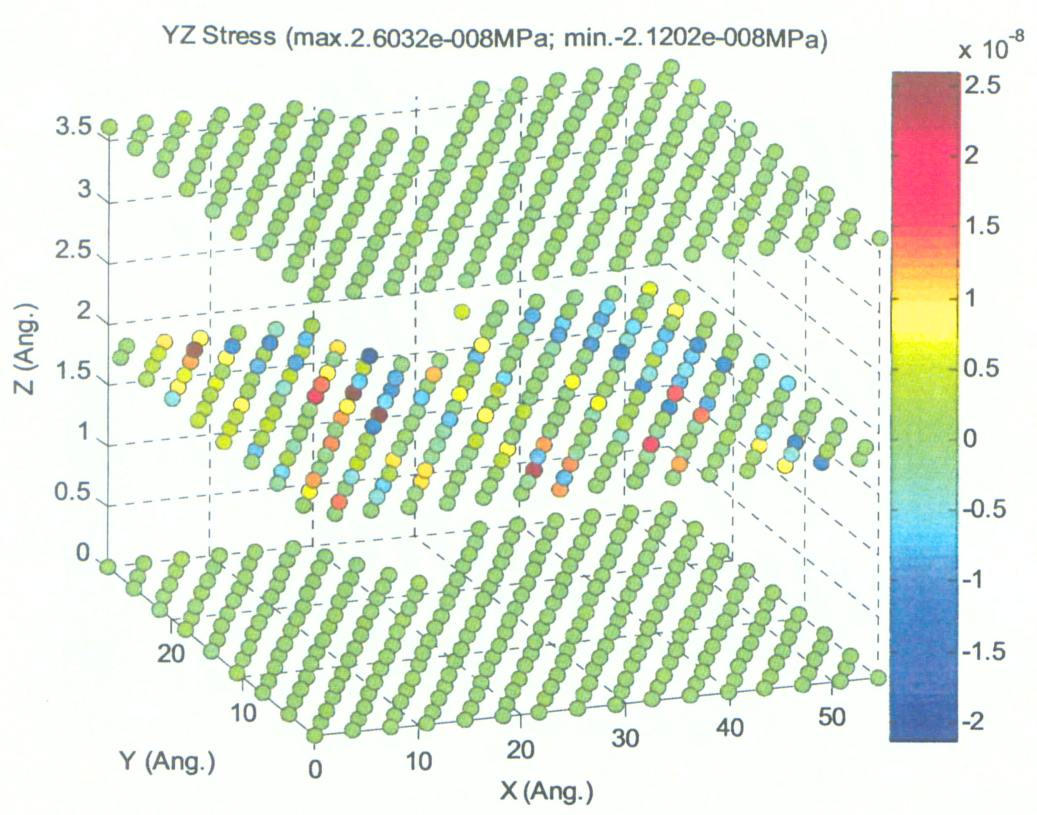

Figure 53 - Application 2: YZ (ZY) Stress Increment from FE-MD Bridged Simulation (MPa)

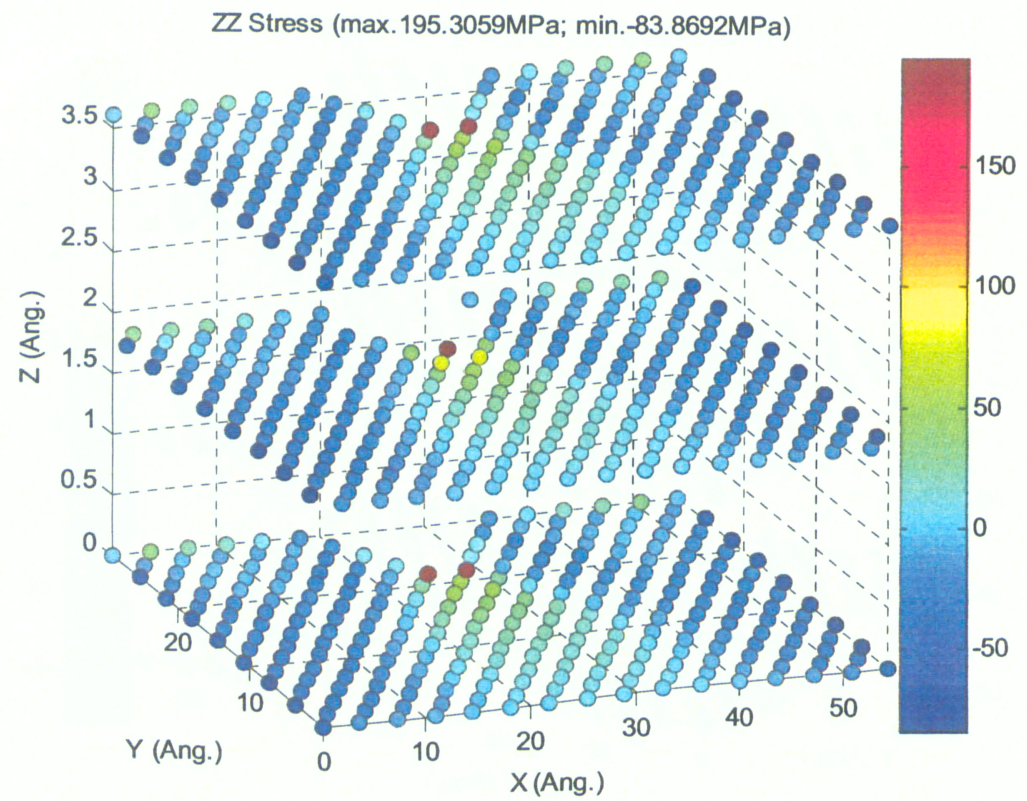

Figure 54 - Application 2: ZZ Stress Increment from FE-MD Bridged Simulation (MPa) 
Table 5 compares the results from the bridged FE-MD method to those from the pure MD simulation (Section 4.3.4).

Table 5 - Summary and Comparison of Results for Application 2

\begin{tabular}{|l|c|c|c|c|c|c|}
\hline \multirow{2}{*}{$\begin{array}{c}\text { Quantity of } \\
\text { Interest }\end{array}$} & \multicolumn{2}{c|}{$\begin{array}{c}\text { Pure MD } \\
\text { Simulation }\end{array}$} & \multicolumn{2}{c|}{$\begin{array}{c}\text { Proposed FE-MD } \\
\text { Bridged Method }\end{array}$} & \multicolumn{2}{c|}{$\begin{array}{c}\text { Difference } \\
\text { (MPa, not \%) }\end{array}$} \\
\cline { 2 - 7 } & Max. & Min. & Max. & Min. & Max. & Min. \\
\hline $\begin{array}{l}\text { XX Virial Stress } \\
\text { Increment (MPa) }\end{array}$ & 276.7362 & -4.7139 & 268.4213 & -4.735 & 8.3149 & 0.0211 \\
\hline $\begin{array}{l}\text { XY Virial Stress } \\
\text { Increment (MPa) }\end{array}$ & 71.4811 & -71.4811 & 69.2885 & -69.2885 & 2.1926 & 2.1926 \\
\hline $\begin{array}{l}\text { XZ Virial Stress } \\
\text { Increment (MPa) }\end{array}$ & Negligible & Negligible & Negligible & Negligible & N/A & N/A \\
\hline $\begin{array}{l}\text { YY Virial Stress } \\
\text { Increment (MPa) }\end{array}$ & 91.2001 & -75.8637 & 88.5507 & -71.9483 & 2.6494 & 3.9154 \\
\hline $\begin{array}{l}\text { YZ Virial Stress } \\
\text { Increment (MPa) }\end{array}$ & Negligible & Negligible & Negligible & Negligible & N/A & N/A \\
\hline $\begin{array}{l}\text { ZZ Virial Stress } \\
\text { Increment (MPa) }\end{array}$ & 200.4398 & -85.4658 & 195.3059 & -83.8692 & 5.1339 & 1.5966 \\
\hline Simulation Time & \multicolumn{2}{|c|}{1 hour } & 45 minutes & & N/A \\
\hline
\end{tabular}

\subsection{Application 3: Elastically Stressed Infinite, Elliptical Void in a Perfect Copper Crystal Lattice}

The results of the bridged FE-MD analysis using FE_MD_V_7_00 for this case are shown in Figure 55 through Figure 66. 


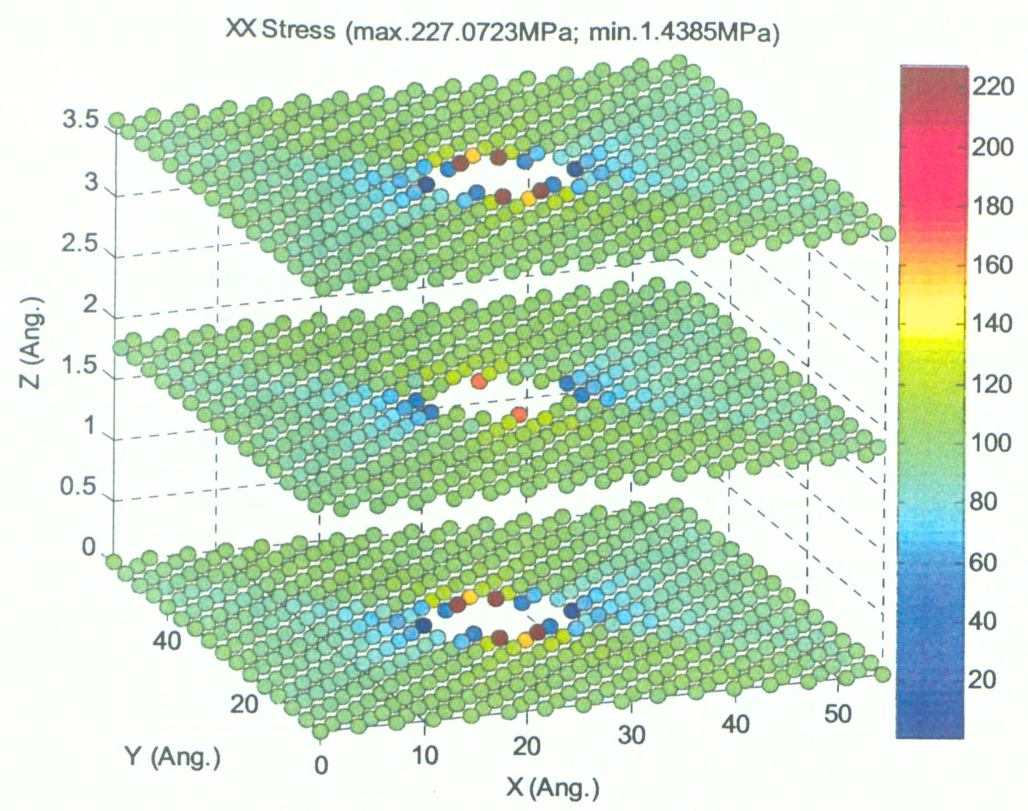

Figure 55 - Application 3: XX Stress Increment from FE-MD Bridged Simulation (MPa) for 100MPa XX Applied Far-Field Stress

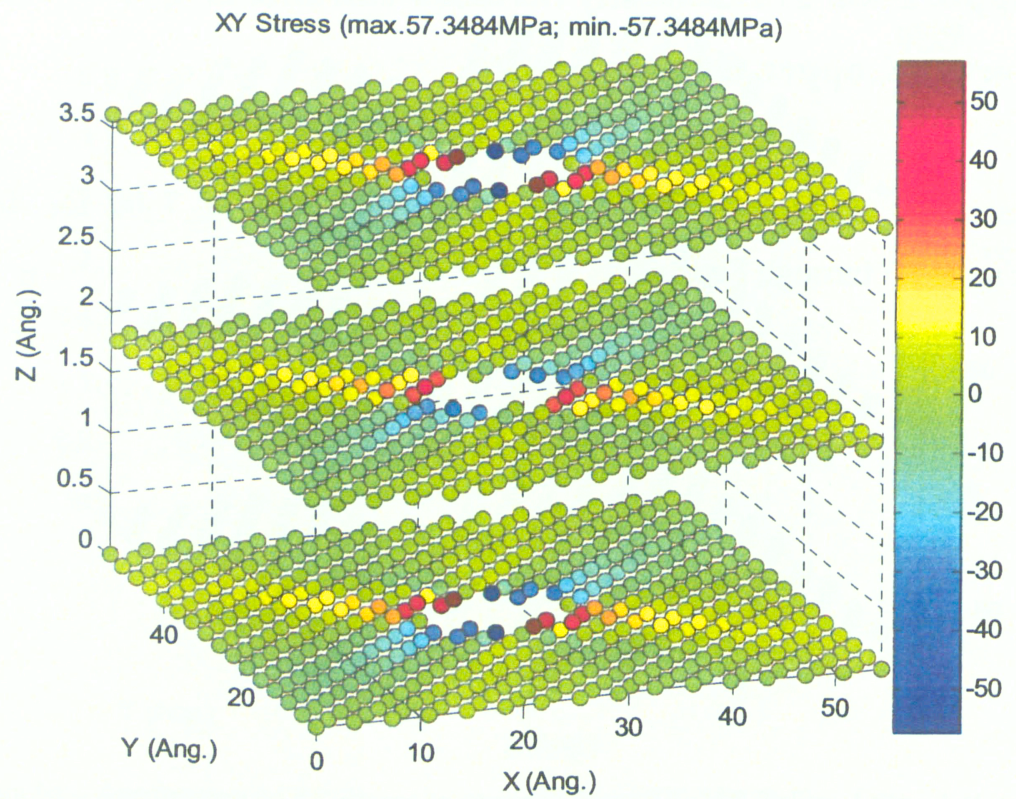

Figure 56 - Application 3: XY (YX) Stress Increment from FE-MD Bridged Simulation (MPa) for 100MPa XX Applied Far-Field Stress 


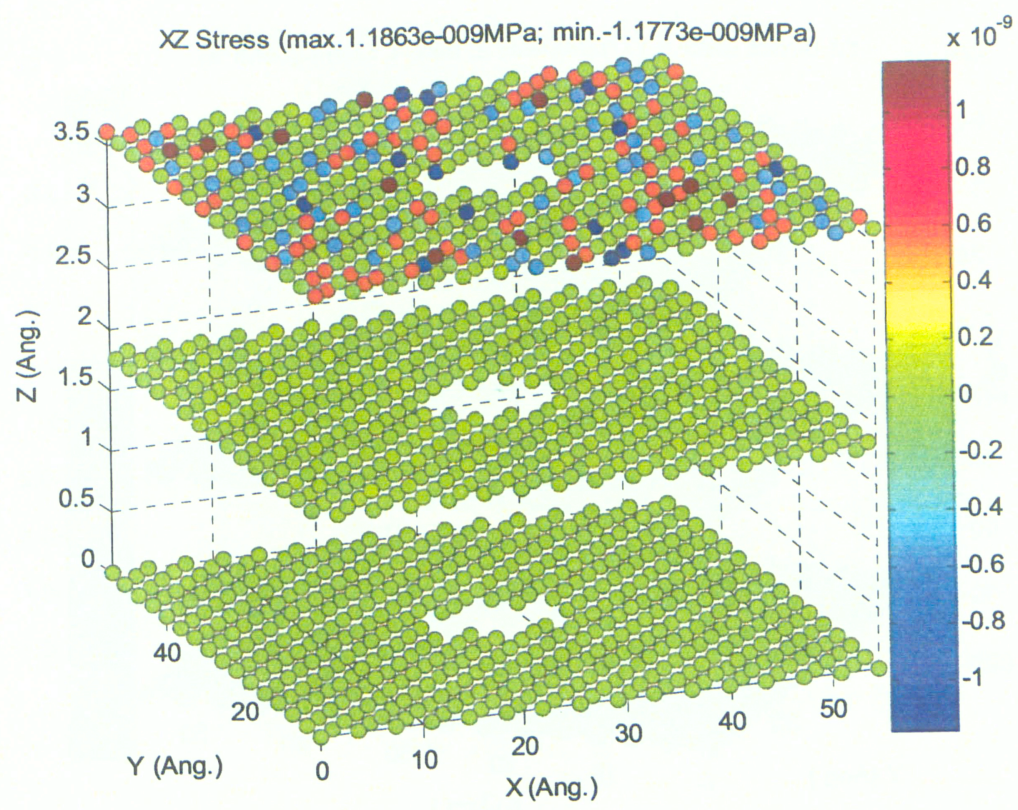

Figure 57 - Application 3: XZ (ZX) Stress Increment from FE-MD Bridged Simulation (MPa) for 100MPa XX Applied Far-Field Stress

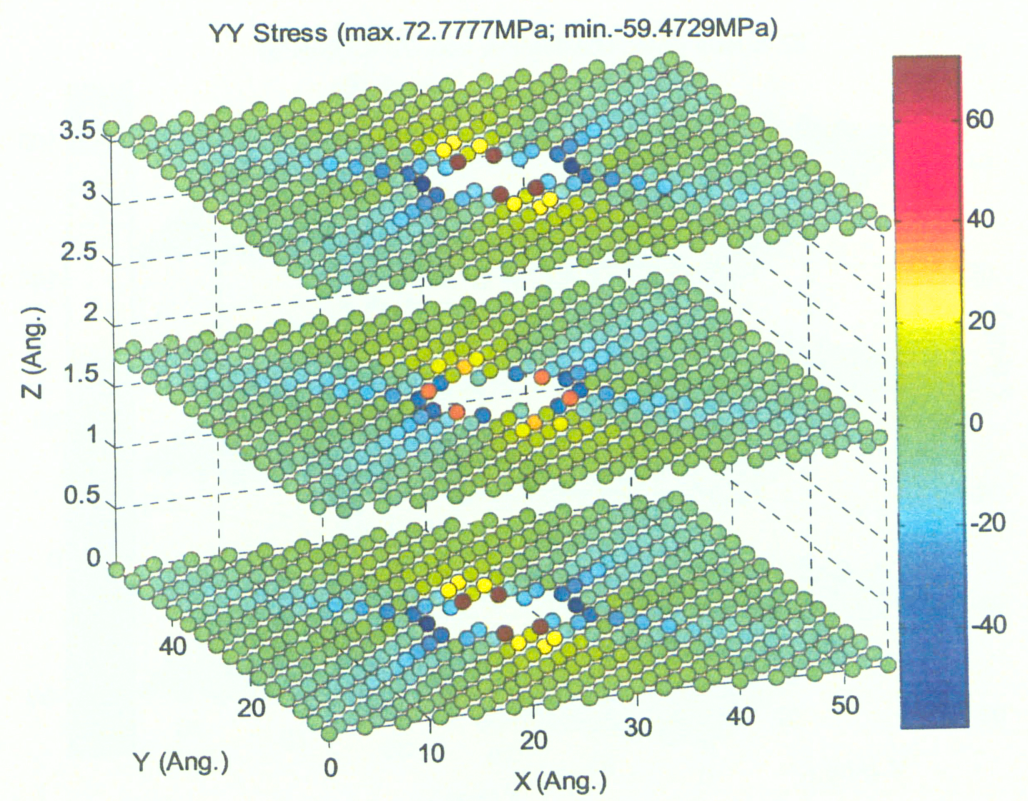

Figure 58 - Application 3: YY Stress Increment from FE-MD Bridged Simulation (MPa) for 100MPa XX Applied Far-Field Stress 


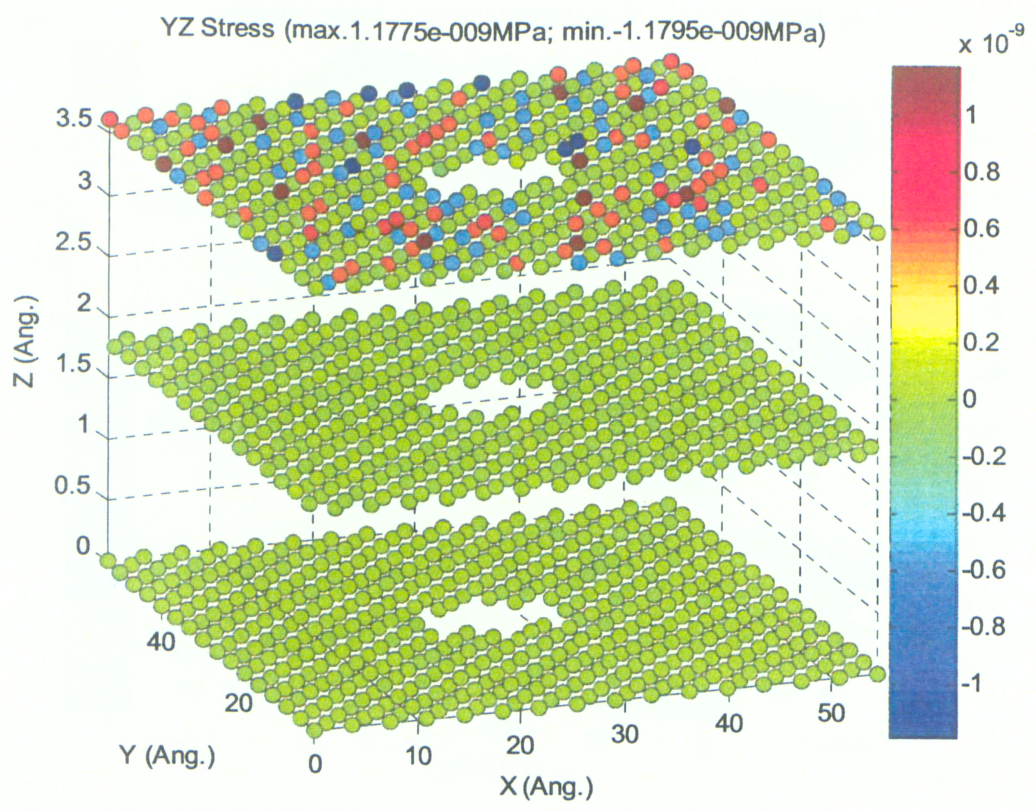

Figure 59-Application 3: YZ (ZY) Stress Increment from FE-MD Bridged Simulation (MPa) for 100MPa XX Applied Far-Field Stress

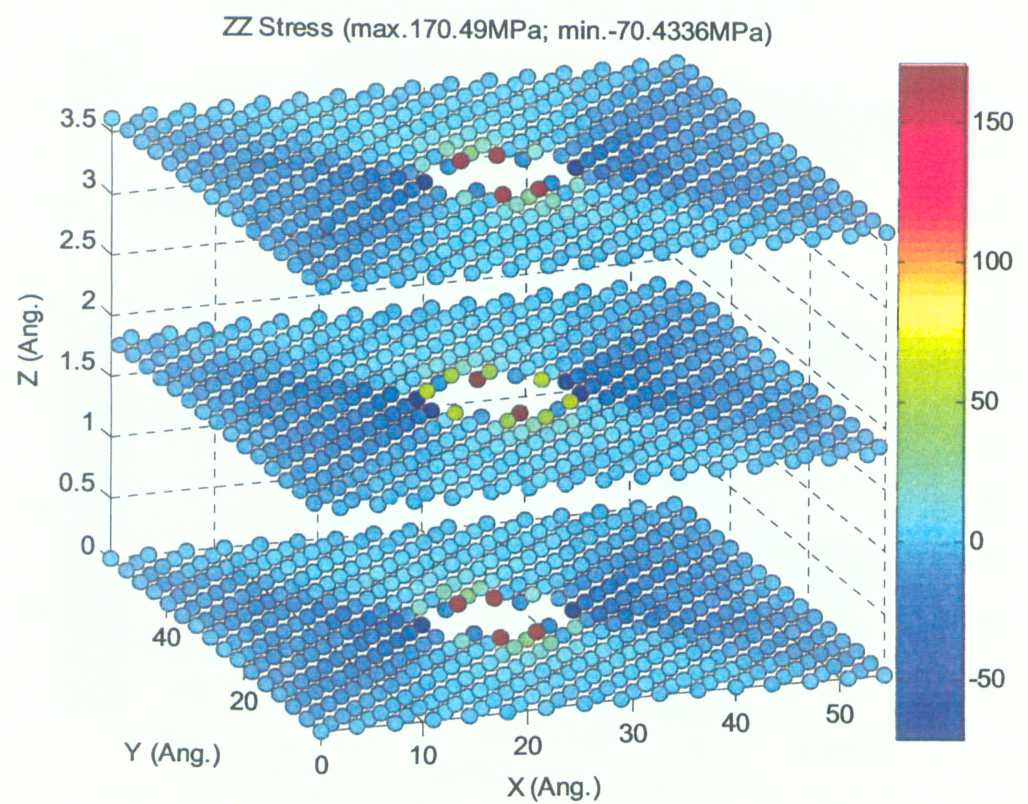

Figure 60 - Application 3: ZZ Stress Increment from FE-MD Bridged Simulation (MPa) for 100MPa XX Applied Far-Field Stress 


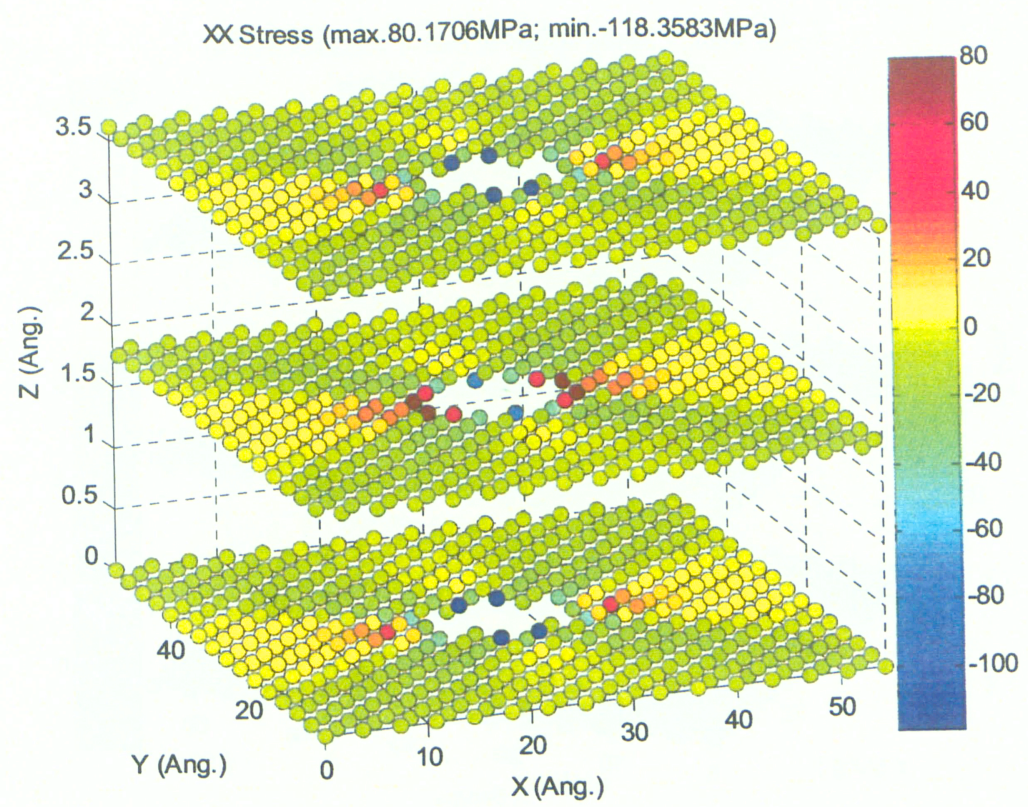

Figure 61 - Application 3: XX Stress Increment from FE-MD Bridged Simulation (MPa) for 100MPa YY Applied Far-Field Stress

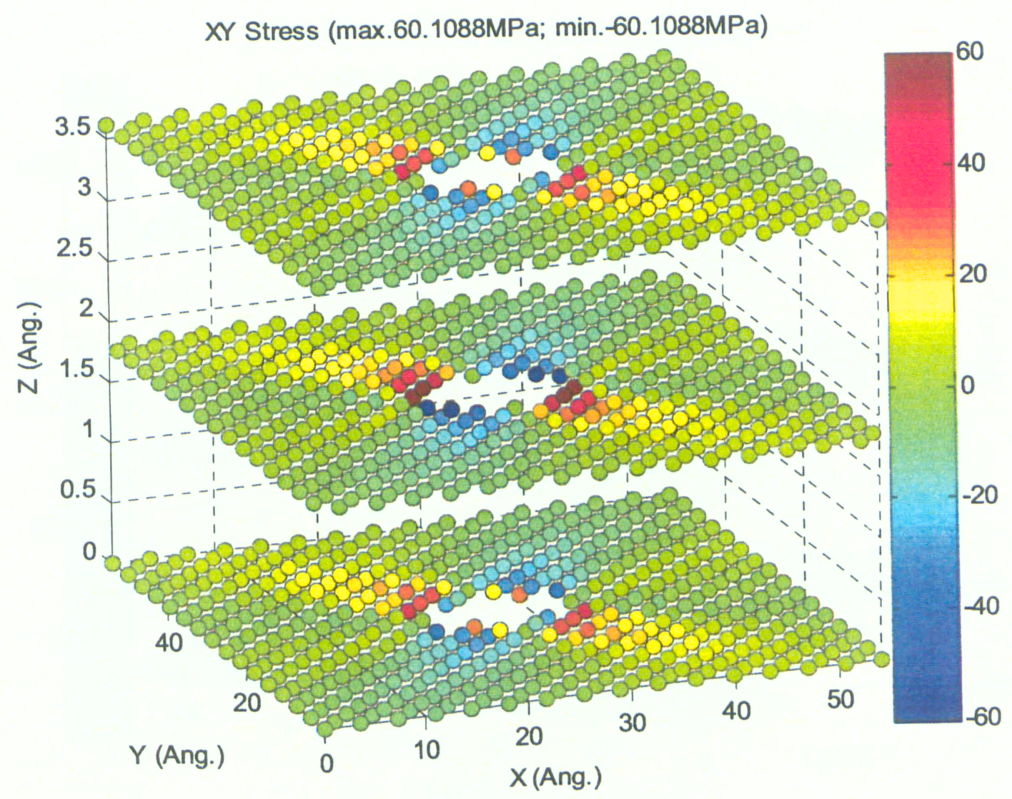

Figure 62 - Application 3: XY (YX) Stress Increment from FE-MD Bridged Simulation (MPa) for 100MPa YY Applied Far-Field Stress 


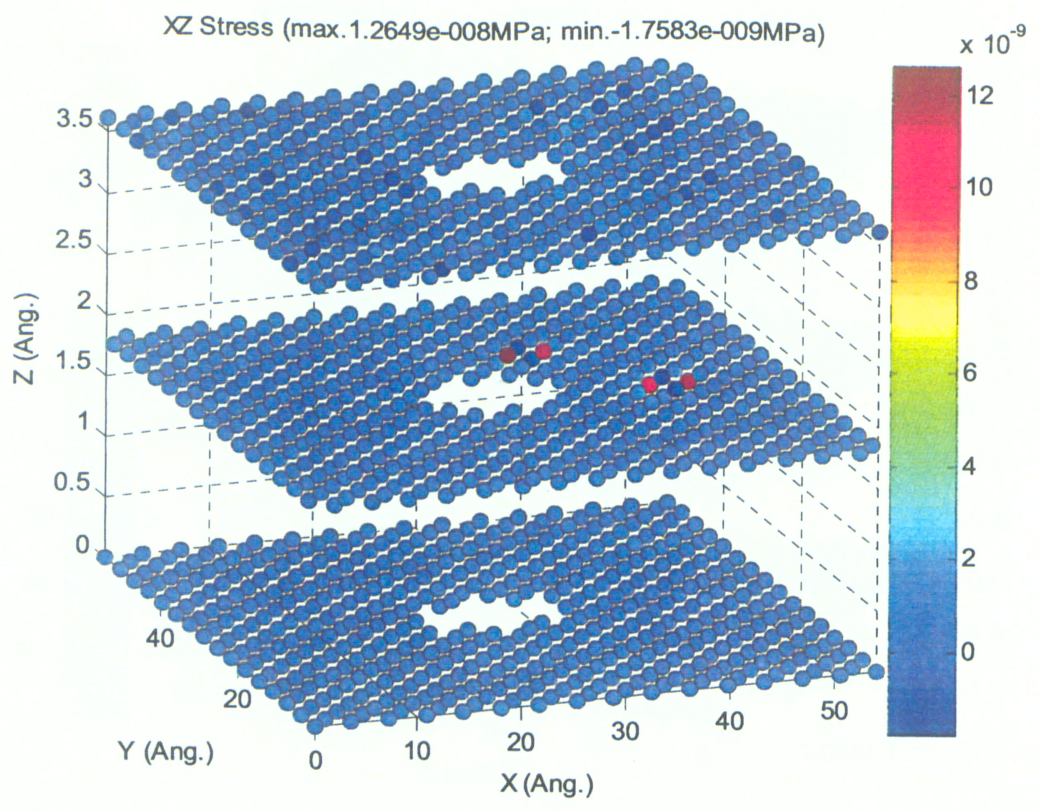

Figure 63 - Application 3: XZ (ZX) Stress Increment from FE-MD Bridged Simulation (MPa) for 100MPa YY Applied Far-Field Stress

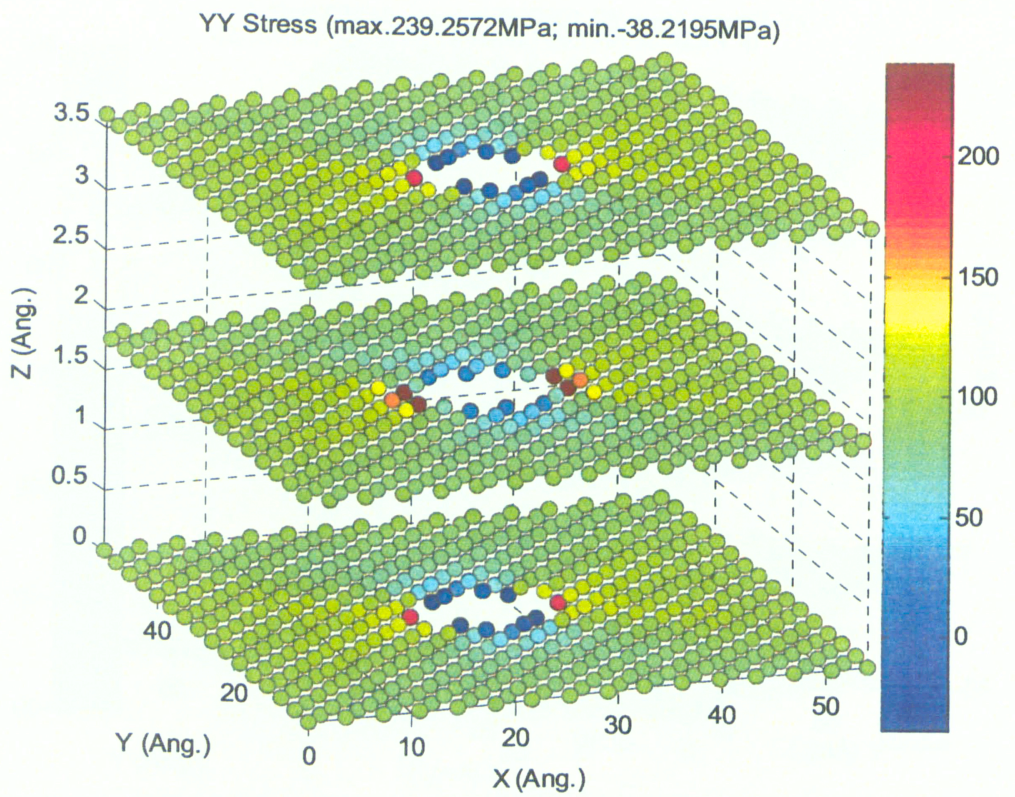

Figure 64 - Application 3: YY Stress Increment from FE-MD Bridged Simulation (MPa) for 100MPa YY Applied Far-Field Stress 


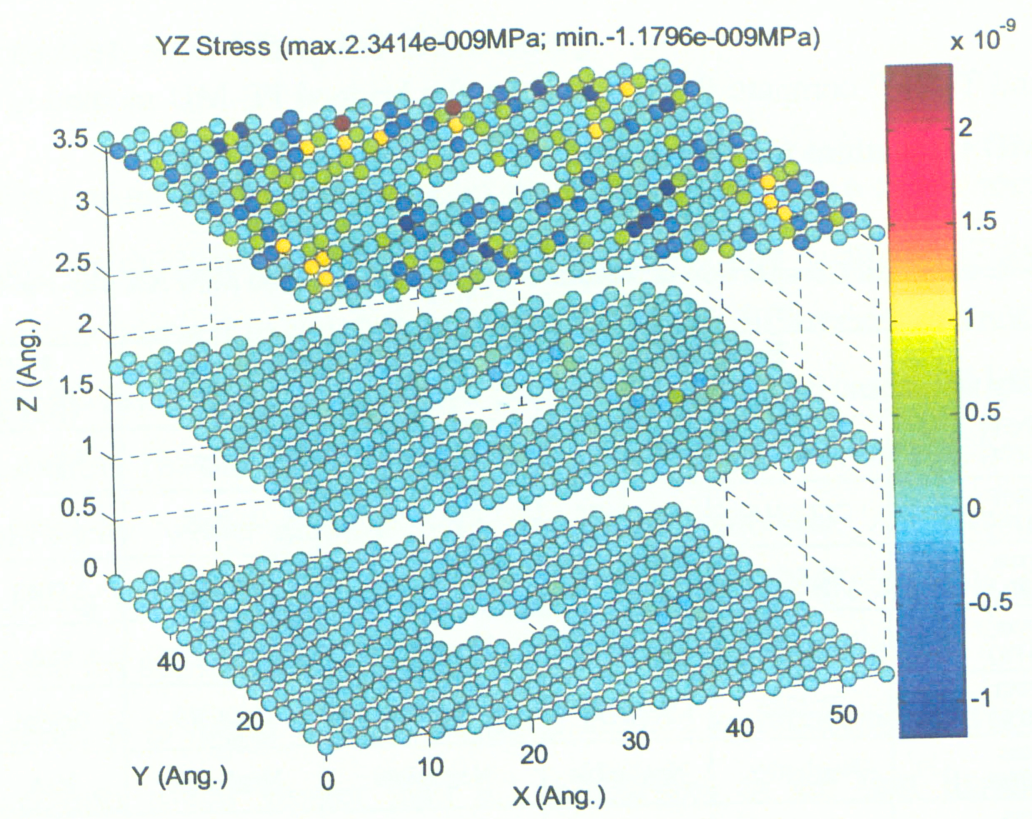

Figure 65 - Application 3: YZ (ZY) Stress Increment from FE-MD Bridged Simulation (MPa) for 100MPa YY Applied Far-Field Stress

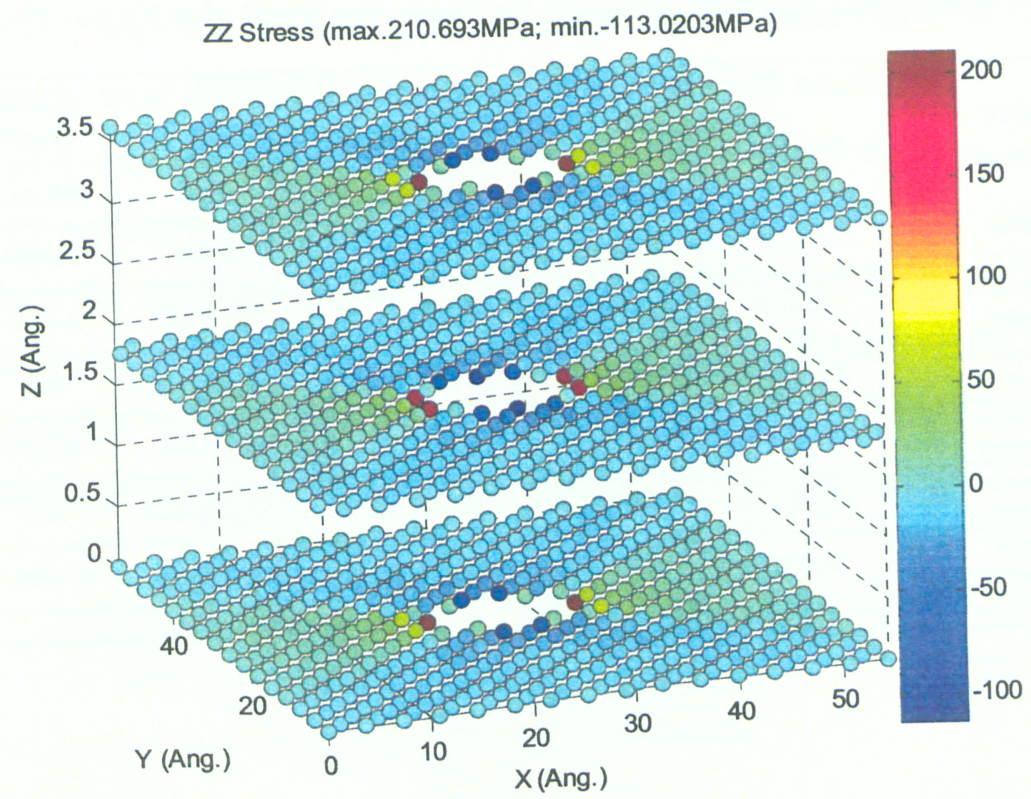

Figure 66 - Application 3: ZZ Stress Increment from FE-MD Bridged Simulation (MPa) for 100MPa YY Applied Far-Field Stress 
Table 6 and Table 7 compare the results from the bridged FE-MD method to those from the pure MD simulations (Section 4.4.4).

Table 6 - Summary and Comparison of Results for Application 3 (100 MPa XX Far-Field Stress)

\begin{tabular}{|l|c|c|c|c|c|c|}
\hline \multirow{2}{*}{$\begin{array}{c}\text { Quantity of } \\
\text { Interest }\end{array}$} & \multicolumn{2}{c|}{$\begin{array}{c}\text { Pure MD } \\
\text { Simulation }\end{array}$} & \multicolumn{2}{c|}{$\begin{array}{c}\text { Proposed FE-MD } \\
\text { Bridged Method }\end{array}$} & \multicolumn{2}{c|}{$\begin{array}{c}\text { Difference } \\
\text { (MPa, not \%) }\end{array}$} \\
\cline { 2 - 7 } & Max. & Min. & Max. & Min. & Max. & Min. \\
\hline $\begin{array}{l}\text { XX Virial Stress } \\
\text { Increment (MPa) }\end{array}$ & 222.5718 & -0.21575 & 227.0723 & 1.4385 & 4.5005 & 1.6543 \\
\hline $\begin{array}{l}\text { XY Virial Stress } \\
\text { Increment (MPa) }\end{array}$ & 51.4421 & -51.4421 & 57.3484 & -57.3484 & 5.9063 & 5.9063 \\
\hline $\begin{array}{l}\text { XZ Virial Stress } \\
\text { Increment (MPa) }\end{array}$ & Negligible & Negligible & Negligible & Negligible & N/A & N/A \\
\hline $\begin{array}{l}\text { YY Virial Stress } \\
\text { Increment (MPa) }\end{array}$ & 72.2283 & -60.9901 & 72.7777 & -59.4729 & 0.5494 & 1.5172 \\
\hline $\begin{array}{l}\text { YZ Virial Stress } \\
\text { Increment (MPa) }\end{array}$ & Negligible & Negligible & Negligible & Negligible & N/A & N/A \\
\hline $\begin{array}{l}\text { ZZ Virial Stress } \\
\text { Increment (MPa) }\end{array}$ & 167.9680 & -69.4095 & 170.4900 & -70.4336 & 2.5220 & 1.0241 \\
\hline Simulation Time & \multicolumn{2}{|c|}{ 2 hours } & \multicolumn{2}{c|}{15 minutes } & \multicolumn{2}{c|}{ N/A } \\
\hline
\end{tabular}

Table 7 - Summary and Comparison of Results for Application 3 (100 MPa YY Far-Field Stress)

\begin{tabular}{|l|c|c|c|c|c|c|}
\hline \multirow{2}{*}{$\begin{array}{c}\text { Quantity of } \\
\text { Interest }\end{array}$} & \multicolumn{2}{c|}{$\begin{array}{c}\text { Pure MD } \\
\text { Simulation }\end{array}$} & \multicolumn{2}{c|}{$\begin{array}{c}\text { Proposed FE-MD } \\
\text { Bridged Method }\end{array}$} & \multicolumn{2}{c|}{$\begin{array}{c}\text { Difference } \\
\text { (MPa, not \%) }\end{array}$} \\
\cline { 2 - 7 } & Max. & Min. & Max. & Min. & Max. & Min. \\
\hline $\begin{array}{l}\text { XX Virial Stress } \\
\text { Increment (MPa) }\end{array}$ & 77.2217 & -90.9001 & 80.1706 & -118.3583 & 2.9489 & 27.4582 \\
\hline $\begin{array}{l}\text { XY Virial Stress } \\
\text { Increment (MPa) }\end{array}$ & 57.4148 & -57.4148 & 60.1088 & -60.1088 & 2.6940 & 2.6940 \\
\hline $\begin{array}{l}\text { XZ Virial Stress } \\
\text { Increment (MPa) }\end{array}$ & Negligible & Negligible & Negligible & Negligible & N/A & N/A \\
\hline $\begin{array}{l}\text { YY Virial Stress } \\
\text { Increment (MPa) }\end{array}$ & 238.5373 & -8.0669 & 239.2572 & -38.2195 & 0.7199 & 30.1526 \\
\hline $\begin{array}{l}\text { YZ Virial Stress } \\
\text { Increment (MPa) }\end{array}$ & Negligible & Negligible & Negligible & Negligible & N/A & N/A \\
\hline $\begin{array}{l}\text { ZZ Virial Stress } \\
\text { Increment (MPa) }\end{array}$ & 188.7929 & -104.8775 & 210.6930 & -113.0203 & 21.9001 & 8.1428 \\
\hline Simulation Time & \multicolumn{2}{|c|}{ 2 hours } & \multicolumn{2}{c|}{15 minutes } & \multicolumn{2}{c|}{ N/A } \\
\hline
\end{tabular}




\subsection{Discussion and Chapter Summary}

The results of the proposed FE-MD bridged method are very close to the results of pure MD simulations, as evident from the data presented in Table 4 through Table 7. Some differences can be observed in the data of Table 7, but the differences are not very large. In fact, the FE-MD bridged method is more conservative in these cases, which means that it can be used safely (in the stress engineering sense). The difference is because of the inherent nonlinearity while dealing with the EAM potential. An iterative method involving updating the stiffness matrix after each step to account for this non-linearity was implemented for application 2 , which is why it took longer to run.

In general, it is found that the iterative method is recommended for maximum nodal displacements much greater than 0.1 Angstroms in the first iteration. This is because the non-linearity of the EAM potential becomes substantial at large displacements, and is not accounted for in a single-step linear FE-MD simulation. However, otherwise, the FE-MD bridged method results are very close to the MD simulation results, with the FE-MD bridged results being conservative in cases where there is a relatively large difference in stresses between the two methods.

Also, as evident from the last entry in each of Table 4 through Table 7, the bridged FE-MD method is much quicker than MD simulation alone when no iterating is involved. Only a few cases are expected to require iterations, and hence the bridged FEMD method seems capable of analyzing large atomic systems very efficiently.

In conclusion, the proposed FE-MD bridged method is extremely accurate, and looks very promising as an alternative to time-consuming MD simulations. 


\section{CONCLUDING REMARKS}

This thesis has attempted to provide the computational tools necessary to study behaviour of metals at the atomic level. A custom MD code has been written, which can be tailored for any future application. The thesis has also provided a means for creating EAM interatomic potentials for use with the custom MD code, the resulting potential of which has been found to predict thermal and elastic properties of pure FCC copper very well. The procedure is applicable to any FCC or BCC metal, so long as the metal is pure (i.e. contains no alloying elements or impurity atoms). A repository of EAM potentials for various pure metals is a crucial first step in any future work, and the EAM fitting procedure from this thesis will allow such a repository to be easily created. Essentially, the thesis has laid the groundwork for conducting computational research on FCC and BCC metal crystal lattices.

A bridged FE-MD method with a novel element discretization scheme proposed by the author has also been developed. Bridged FE-MD methods constitute one of the several groups of methods being currently developed throughout the world to efficiently analyze large atomic systems. The linear stiffness formulation of the method is ideally suited for atomic systems that are expected to approach a state of equilibrium from an arbitrary initial state (like all the applications in this thesis). The element discretization scheme proposed for this thesis is a very general and powerful scheme which can be applied to any system of discrete point masses interacting via any potential. The results from the bridged method have been found to agree with MD simulation results very well. That being said the reason for developing the bridged method is to analyze atomic systems much more efficiently (i.e. much quicker) than MD simulation alone. This is indeed found to be the case, with the bridged method being up to eight times faster than MD simulation in the applications considered for this thesis.

For systems showing large atomic displacements in the initial FE-MD solution or involving highly nonlinear potentials, an iterative procedure can be employed that entails recalculating the stiffness matrix after each iteration and then recalculating the 
displacements. The iterative procedure is terminated when all displacements fall below a threshold value. This iterative procedure was carried out for one of the applications, and it still proved to be more than twice as fast as the corresponding MD simulation. The bridged method thus promises to be a far more efficient method for analyzing large atomic systems than MD simulation alone.

Note that all applications investigated in this thesis employed the standard [100][010]-[001] crystallographic coordinate system for cubic metals. The reason for doing this was to provide three mutually perpendicular directions that are identical as far as mechanical properties are concerned. This simplified the analysis and programming tremendously. More complicated crystal structures will require consideration of possible anisotropy in the arbitrary coordinate system chosen for the analysis. The kernel of the MD code (the N-body force-equation integrator), however, can be used with any crystal structure.

Only a small portion of the science of atomistic simulations has been investigated in this thesis. There are several potential avenues of work that can be pursued using the results of this thesis. Some of them are: studying dislocation behaviour in real metal crystals; predicting fatigue and fracture behaviour of metals at the atomic level; studying metallurgical processes and phenomena like heat treatment and phase transformation of metals; directly studying the effects of alloying elements on the behaviour of metals; etc. Important work remains to be done to bring the necessary level of maturity to the research group for pursuing these and other challenging endeavours. For example, as mentioned earlier, a repository of EAM potentials for metals and other suitable potentials for non-metals is required to analyze materials of practical engineering significance (like alloys and polymers). Another example is the constant revision of the MD code to utilize available computational resources efficiently. The author hopes that his research work paves the way for all these tasks and years of important and exciting research in the field nanotechnology. 


\section{APPENDIX A: PROGRAM EXECUTION FLOWCHARTS FOR CUSTOM MOLECULAR DYNAMICS (MD) CODE}




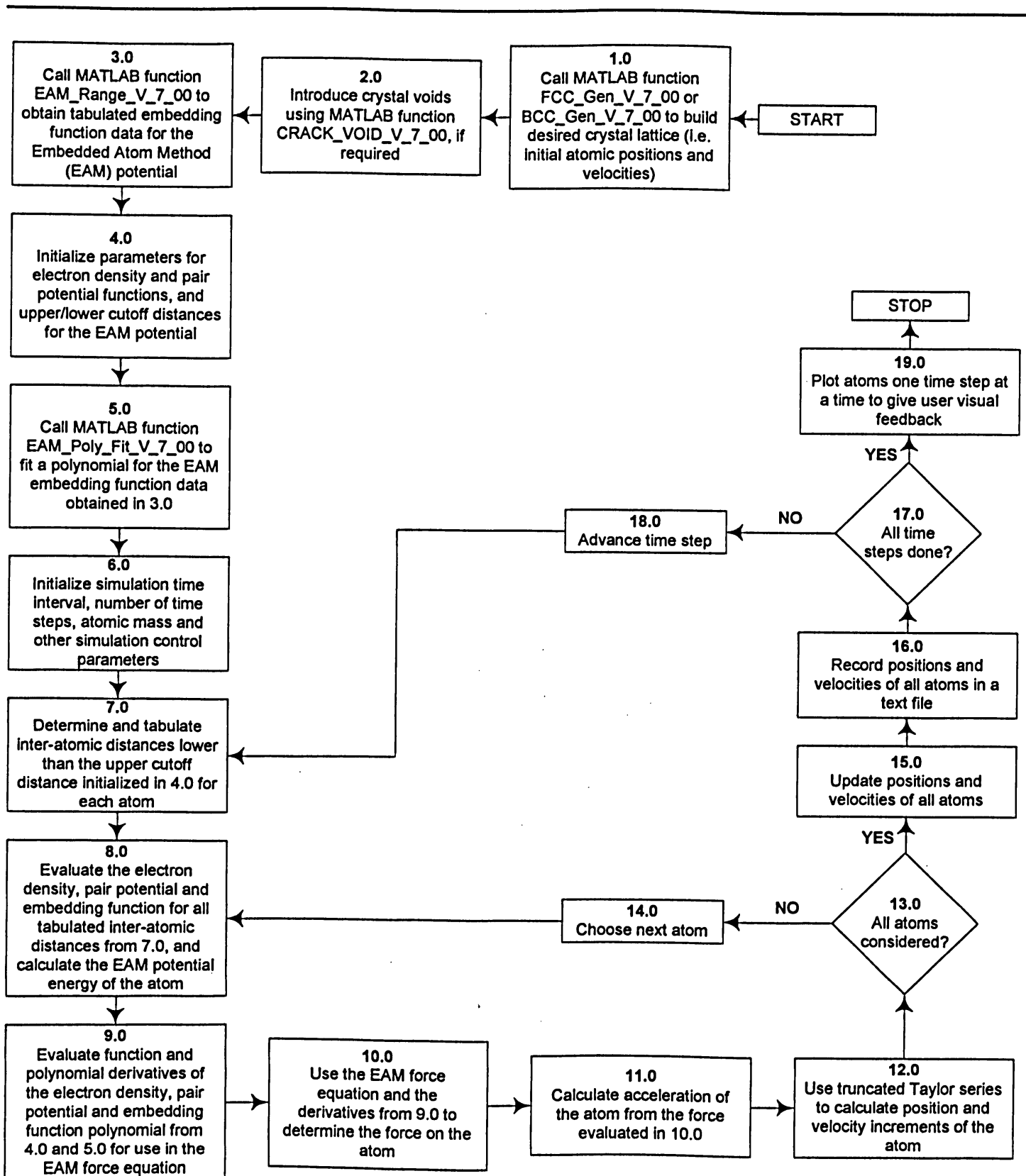

Figure 67 - Program Execution Flowchart for Master MD Function MD_V_7_00 


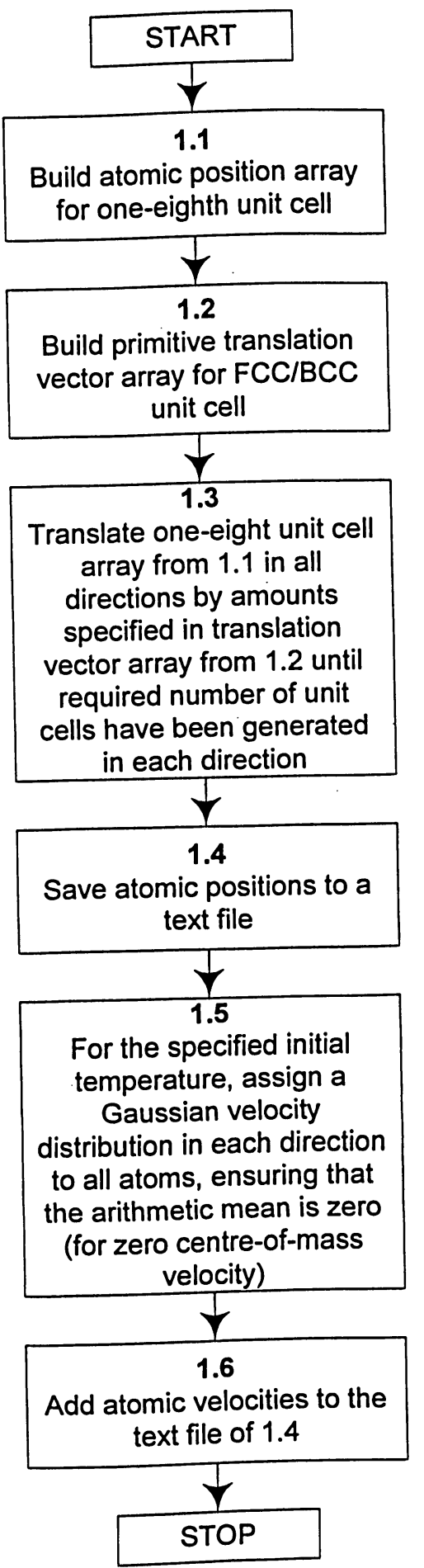

Figure 68 - Program Execution Flowchart for Function FCC_Gen_V_7_00 and BCC_Gen_V_7_00 


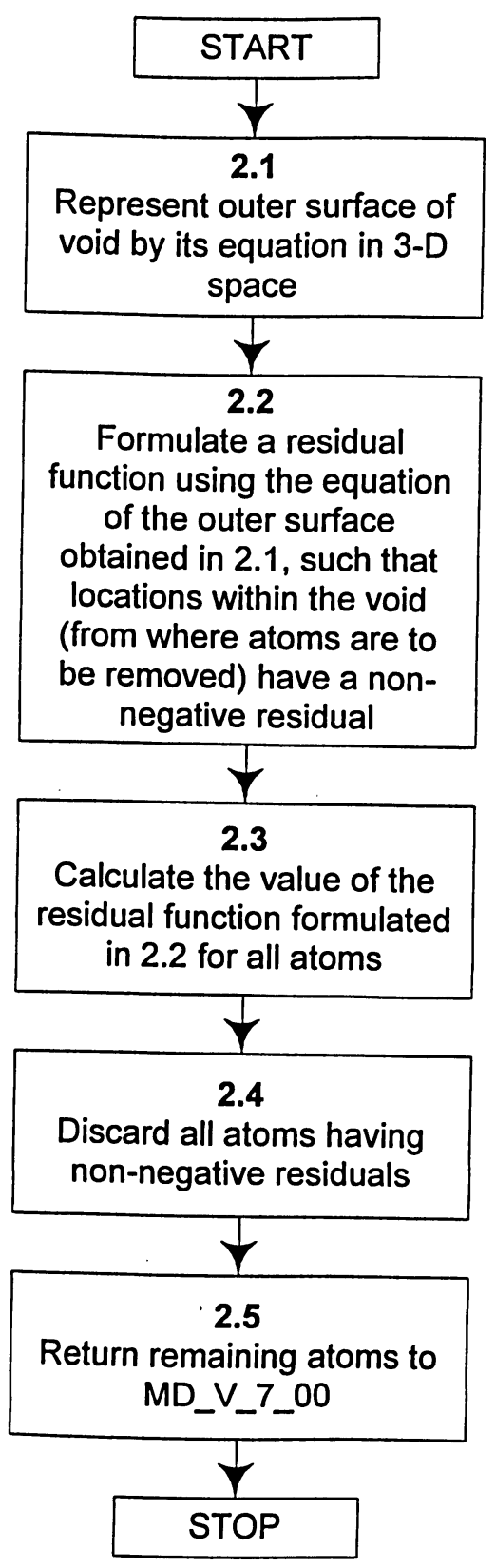

Figure 69 - Program Execution Flowchart for Function CRACK_VOID_V_7_00 


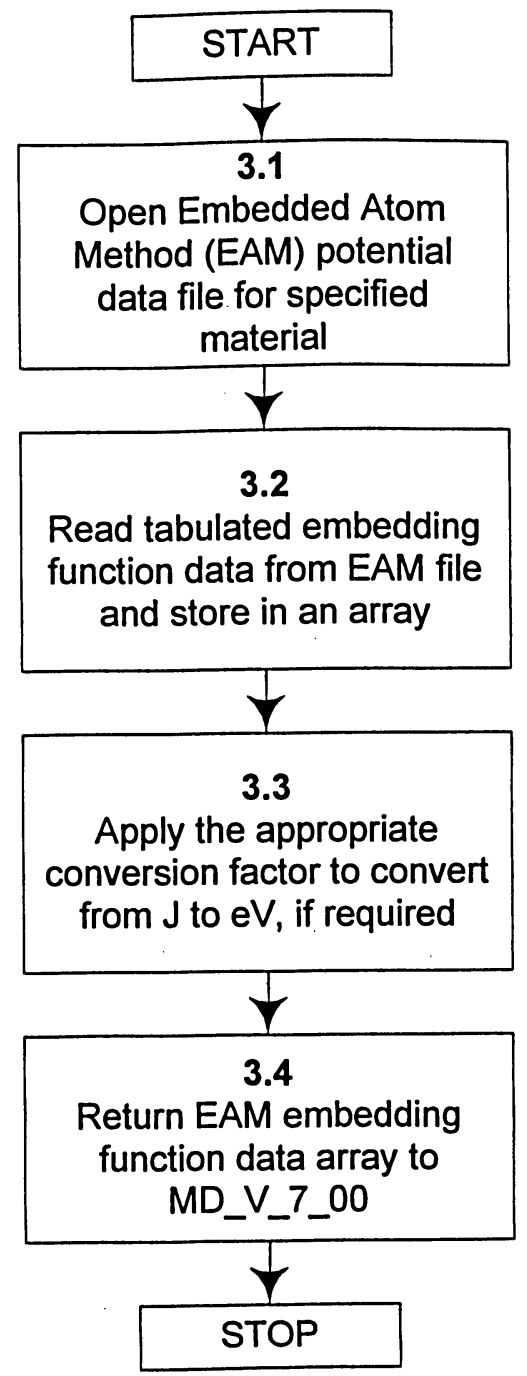

Figure 70 - Program Execution Flowchart for Function EAM_Range_V_7_00 


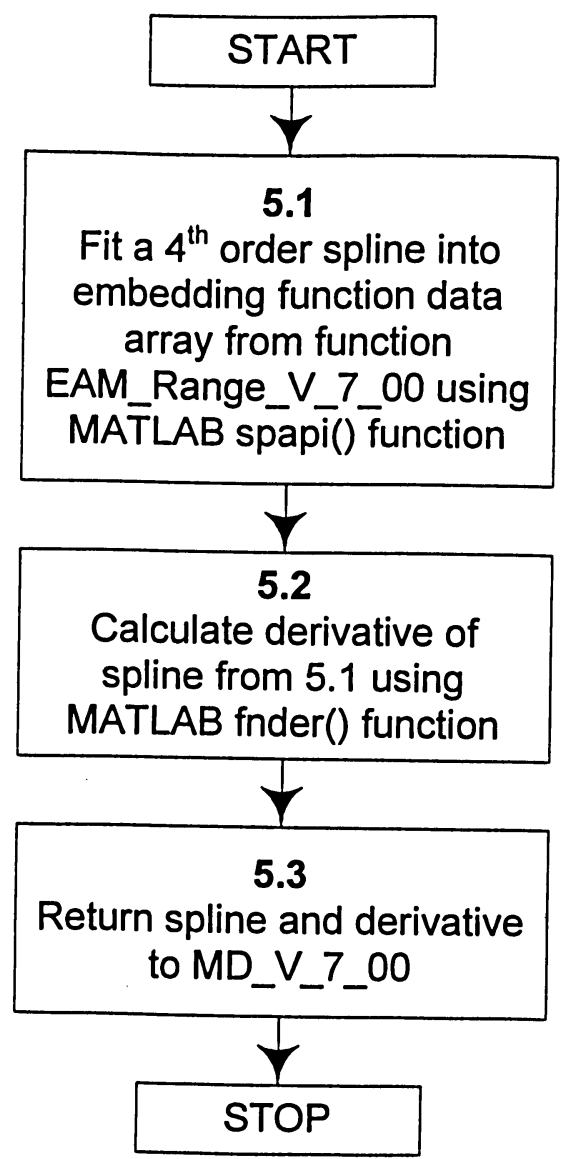

Figure 71 - Program Execution Flowchart for Function EAM_Poly_Fit_V_7_00 


\section{APPENDIX B: INITIAL AND FINAL ENTRIES IN OUTPUT FILE FOR MD_V_7_00 TEST CASE (MOMENTUM AND ENERGY CONSERVATION FOR SINGLE UNIT CELL)}




\section{Initial Entry}

Total simulation time:1.000000e-015 seconds

Individual atoms (coord. in Angstroms, vel. in $\mathrm{m} / \mathrm{s}$ ):

$\begin{array}{lllll}\text { X Coord. } & \text { Y Coord. } & \text { Z Coord. } & \text { X Vel. } & \text { Y Vel. } \\ 0.000034 & 0.000034 & 0.000034 & 6.856990 & 6.856990 \\ 0.000034 & 0.000034 & 3.399966 & 6.856990 & 6.856990 \\ 0.000034 & 3.399966 & 0.000034 & 6.856990 & -6.856990 \\ 0.000034 & 3.399966 & 3.399966 & 6.856990 & -6.856990 \\ 3.399966 & 0.000034 & 0.000034 & -6.856990 & 6.856990 \\ 3.399966 & 0.000034 & 3.399966 & -6.856990 & 6.856990 \\ 3.399966 & 3.399966 & 0.000034 & -6.856990 & -6.856990 \\ 3.399966 & 3.399966 & 3.399966 & -6.856990 & -6.856990 \\ -0.000083 & 1.700000 & 1.700000 & -16.665191 & 0.000000 \\ 3.400083 & 1.700000 & 1.700000 & 16.665191 & 0.000000 \\ 1.700000 & -0.000083 & 1.700000 & 0.000000 & -16.665191 \\ 1.700000 & 3.400083 & 1.700000 & 0.000000 & 16.665191 \\ 1.700000 & 1.700000 & -0.000083 & 0.000000 & 0.000000 \\ 1.700000 & 1.700000 & 3.400083 & 0.000000 & 0.000000\end{array}$

Z Vel. 6.856990

$-6.856990$

6.856990

$-6.856990$

6.856990

$-6.856990$

6.856990

$-6.856990$

0.000000

0.000000

0.000000

0.000000

$-16.665191$

16.665191

System (vel. in $\mathrm{m} / \mathrm{s}$, energy in $\mathrm{eV}$ ) :

COM X Vel. COM Y Vel. COM Z Vel.

$\begin{array}{lll}-0.000000 & 0.000000 & 0.000000\end{array}$

Total PE Total $\mathrm{KE}$

$-32.5498310 .000850$

Total Energy $-32.548981$

Instan. dyn. temp. (K) Avg. dyn. temp. (K) Avg. hydro. stress (MPa)

0.469741

0.469741

2686.905914

Instan. VIRIAL stress tensor (MPa)

$2686.905914 \quad 0.000000 \quad 0.000000$

$0.000000 \quad 2686.905914 \quad 0.000000$

$-0.000000 \quad 0.000000 \quad 2686.905914$

\section{Final Entry}

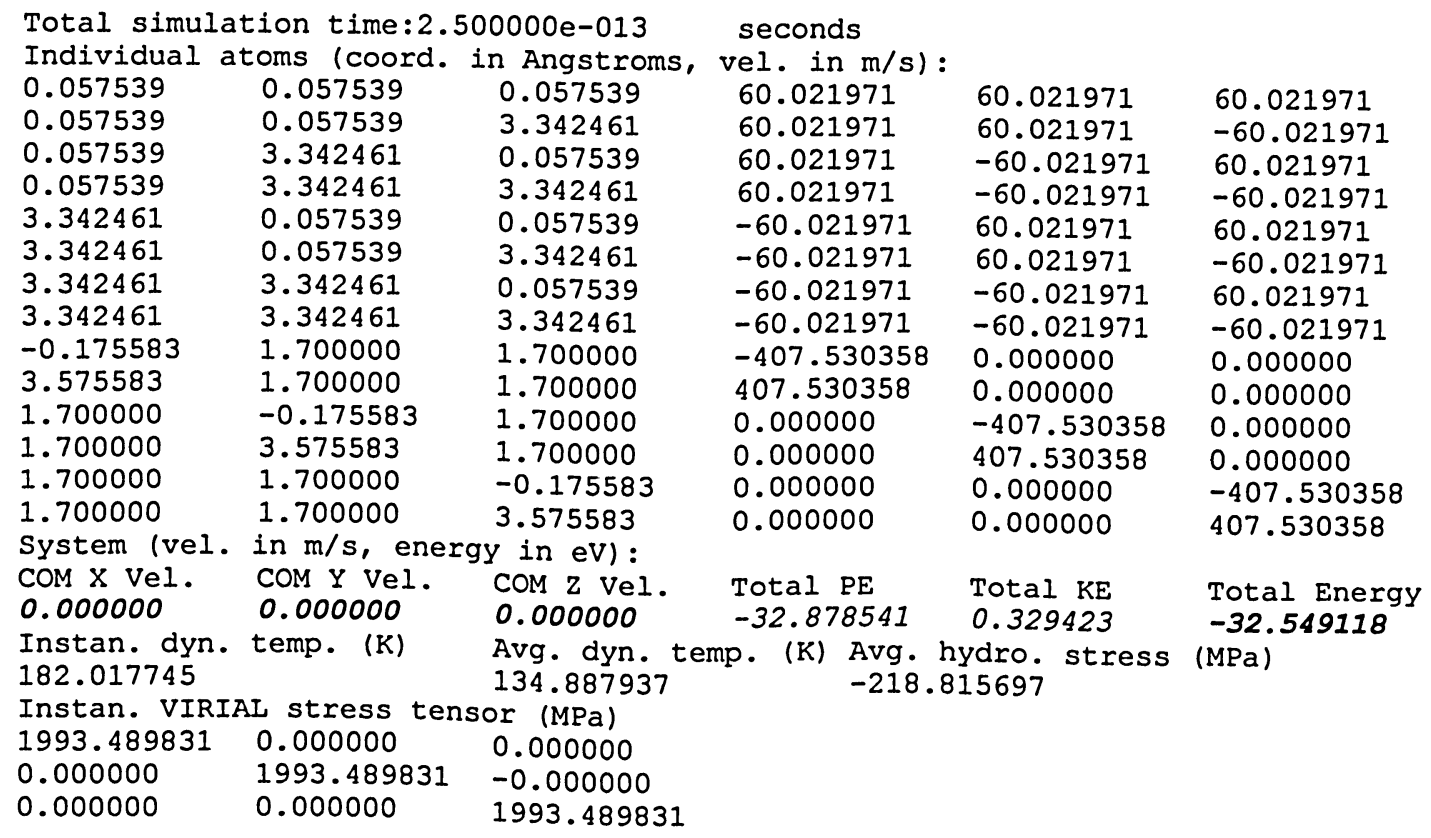




\section{APPENDIX C: FLOWCHART FOR PROPOSED NUMERICAL EMBEDDED ATOM METHOD (EAM) INTERATOMIC POTENTIAL FITTING PROCEDURE}




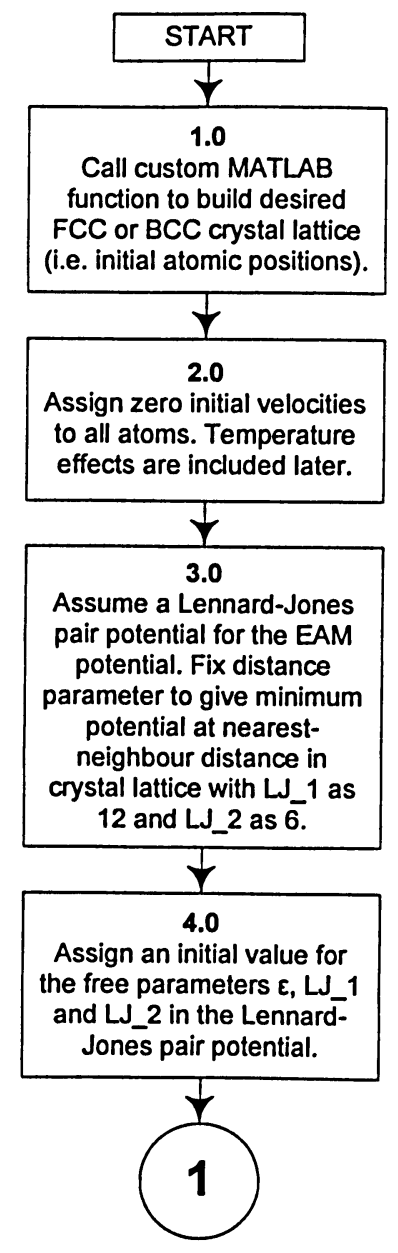

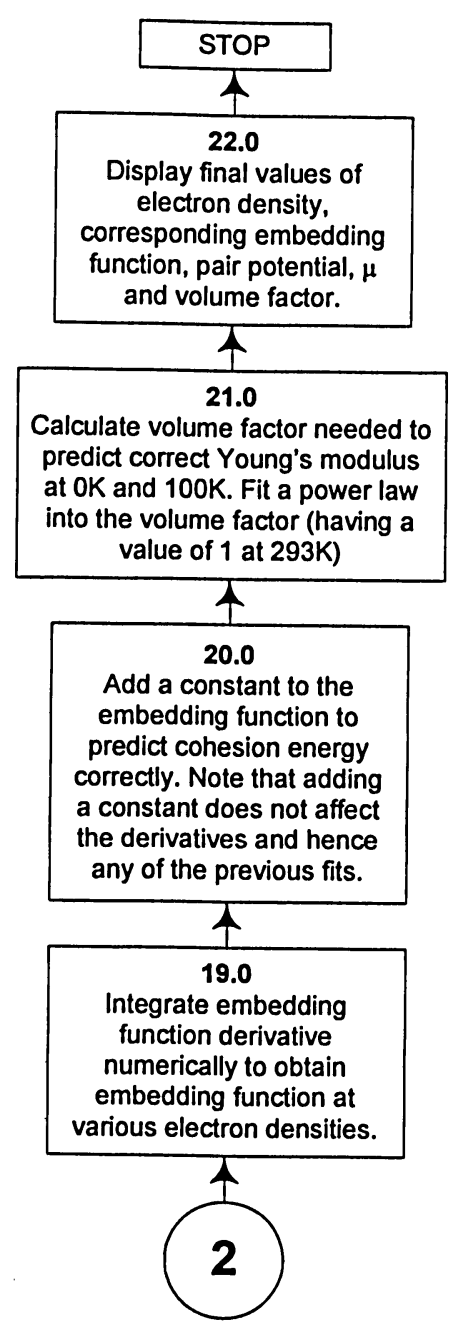

Figure 72 - Flowchart for Proposed Numerical EAM Potential Fitting Procedure (part 1; continued in Figure 73) 


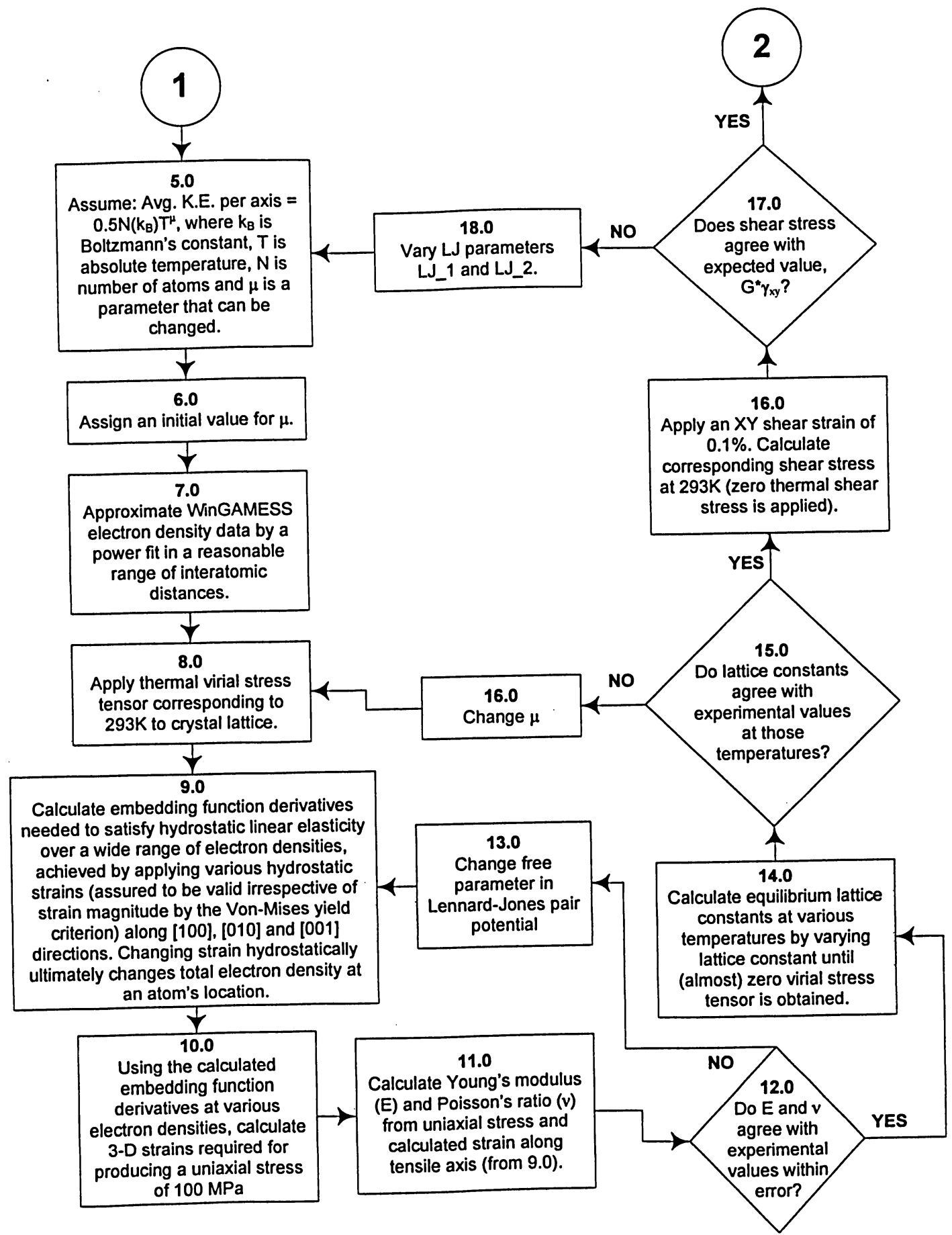

Figure 73 - Flowchart for Proposed Numerical EAM Potential Fitting Procedure (part 2; continued from Figure 72) 


\section{APPENDIX D: A BRIEF OVERVIEW OF QUANTUM MECHANICS}




\section{D1. Introduction}

Quantum mechanics is a branch of science that has been formulated to deal with atomic and sub-atomic particles like nuclei, electrons, etc. Classical mechanics could not explain many of the experimental observations related to atoms and molecules. Quantum mechanics was laid down to explain these experimental observations. Quantum mechanics has its history divided into two different philosophies: the old quantum theory and the new quantum theory. The differences will be summarized in the following sections. Information for this appendix has been obtained from [31].

\section{D2. The Old Quantum Theory}

In 1900, Max Planck explained the discontinuous energy spectrum observed during black-body radiation by postulating that the vibrating particles of matter (considered to act as harmonic oscillators) do not emit or absorb light continuously but instead only in discrete quantities of energy with magnitude hv ( $v$ being the frequency of the light). The constant $h$ was calculated by Planck to be $6.626 \mathrm{e}-34 \mathrm{~kg} \cdot \mathrm{m}^{2} \cdot \mathrm{sec}^{-1}$, and is called Planck's constant. $h v$ was called a quantum of radiant energy of frequency $v$. Planck therefore overcame the conflicting classical result that the radiant energy forms a continuous spectrum by using his postulate of quantization. Thus was born the old quantum theory.

In 1905, Einstein suggested that each quantum of radiant light energy was emitted uni-directionally (like a particle) and not omni-directionally (like a wave). Hence the particle nature of light was suggested, with each light particle dubbed a photon. Using this particle interpretation of light and the quantization postulate of Planck, Einstein explained the photo-electric effect very successfully. Einstein also applied the quantization postulate to a collection of oscillating atoms in a solid material and explained the observed heat-capacities of the solid very successfully. 
The old quantum theory remained at this stage till 1911, when Ernest Rutherford discovered the structure of the atom (a small, heavy, positively charged nucleus surrounded by light, negatively charged electrons bound to the nucleus by Coulomb electrostatic forces). In 1913, Bohr used this model of the Hydrogen atom and explained the observed emission spectra of Hydrogen successfully. During his derivation, Bohr realized that the emission spectra and stationary states (states that correspond to definite values of energy) could be accurately predicted if he quantized the angular momentum of the electron as integral multiples of $h /(2 \pi)$. This was the quantization rule for Bohr's model of the Hydrogen atom.

In 1915, this quantization rule was found to be a special case of a general quantization rule discovered by $\mathrm{W}$. Wilson and A. Sommerfeld, called the WilsonSommerfeld quantization rule. The rule states that a generalized coordinate $\left(q_{k}\right)$ and its canonically conjugate generalized momentum ( $p_{k}$, defined as in Hamiltonian dynamics) are related by the following quantization expression:

$$
\oint p_{k} d q_{k}=n_{k} h, k=1,2, \ldots, 3 n ; n_{k}=\text { an integer called the quantum number }
$$

The integral in equation (D1) is taken over one complete cycle of the motion of the system.

Bohr also laid down a very important principle in quantum mechanics, called the correspondence principle. According to this principle, the classical results and quantum results will numerically converge at large enough values of the quantum number(s) ( $n_{k}$ in equation (D1)).

The old quantum theory slowly declined because of some inadequacies discovered shortly there after. For example, old quantum theory predicted the energy of a one-dimensional harmonic oscillator to be $n h v$ ( $n$ being the quantum number), whereas the actual energy determined from experiments was $(n+0.5) h v$. The old quantum theory was eventually replaced by the new quantum theory, which is the focus of the remaining sections of this appendix. 


\section{D3. The New Quantum Theory}

The new quantum theory was developed in two frameworks which were ultimately proven to be equivalent: wave mechanics which was due to Erwin Schrödinger, and matrix mechanics which was due to Werner Heisenberg. The focus will predominantly be on wave mechanics, which is more intuitive than matrix mechanics.

\section{D4. The General Nature of New Quantum Theory}

It should be understood (and this cannot be reiterated enough) that the new quantum theory is based on a group of postulates that cannot be derived from anything else. The postulates were deductively laid down with the ultimate hope that experiments would confirm any predictions from the postulates. It is the crowning achievement of Erwin Schrödinger's wave mechanics and Werner Heisenberg's matrix mechanics that almost all experimentally observed phenomena at the atomic and sub-atomic levels have successfully been explained using these postulates. Today, the postulates have been validated by experimental observations enough times that the postulates are assumed to be fundamental to the working of nature.

\section{D5. Erwin Schrödinger's Wave Mechanics}

\section{D5.1 Postulates of Wave Mechanics}

\section{Existence of probability densities and amplitudes:}

One of the most important principles in new quantum theory is the uncertainty principle (by Heisenberg and Bohr), which states that it is impossible to simultaneously measure exactly the position and momentum of a small, moving 
particle like an electron. This is easy to see with a thought experiment: assume that one wants to measure the position of an electron exactly. The measurement is effected using a physical apparatus, like a microscope. For one to see the electron through the microscope, the particles of some incident beam must be bounced by the electron into the aperture of the microscope. This very act of measurement will then result in the momentum of the electron changing. Hence the momentum of the electron is uncertain, even though the position is certain.

Given the uncertainty principle, one can no longer talk about the exact position and momentum of a particle, but can only talk about the probability that the particle has a certain position and momentum. The probability that a particle is at a certain position in space can then be expressed by a probability density function, $P\left(q_{1}, q_{2}, \ldots, q_{D}\right)$, the probability being:

$$
\operatorname{Pr} .=P\left(q_{1}, q_{2}, \ldots, q_{f}\right) d q_{1} d q_{2} \ldots d q_{f}
$$

$q_{1}, q_{2}, \ldots, q_{f}$ are the $f$ generalized coordinates of motion of the system. Now, probability is always a real, non-negative quantity that is less than or equal to 1 . To ensure that this is the case (or to reflect this fact), $P\left(q_{1}, q_{2}, \ldots, q_{D}\right)$ is written as the product of a complex quantity $\Psi\left(q_{1}, q_{2}, \ldots, q_{\partial}\right)$ and its conjugate. Recall that the product of a complex number and its conjugate is always non-negative. Hence the following equation is written for $P\left(q_{1}, q_{2}, \ldots, q_{D}\right)$ :

$$
P\left(q_{1}, q_{2}, \ldots, q_{f}\right)=\Psi^{*}\left(q_{1}, q_{2}, \ldots, q_{f}\right) \Psi\left(q_{1}, q_{2}, \ldots, q_{f}\right)
$$

Therefore, the probability of finding a particle in the infinitesimal ranges of positions $q_{1}+d q_{1}, q_{2}+d q_{2}, \ldots, q_{f}+d q_{f}$ is (using equation (D3) in (D2)):

$$
\left.\operatorname{Pr} .=\Psi^{*}\left(q_{1}, q_{2}, \ldots, q_{f}\right) \Psi q_{1}, q_{2}, \ldots, q_{f}\right) d q_{1} d q_{2} \ldots d q_{f}
$$


Now, the particle must be found somewhere in space. In other words, the probability of finding the particle somewhere in space is 1 . This is expressed as:

$$
\int \ldots \int \Psi^{*}\left(q_{1}, q_{2}, \ldots, q_{f}\right) \Psi\left(q_{1}, q_{2}, \ldots, q_{f}\right) d q_{1} d q_{2} \ldots d q_{f}=1
$$

, where each integration is performed over the complete possible range of that variable. Equation (D5) is called the normalization condition. The complex quantity $\Psi$ is called the probability amplitude of the system.

2. Change of $\Psi$ with Time - Schrödinger's Wave Equation Including the Time:

Erwin Schrödinger postulated the following equation for predicting the change of the probability amplitude $\Psi$ of a single particle of mass $m$ in Cartesian coordinates moving in a conservative field with time, based on analogies between $\Psi$ and quantities in classical mechanics. This equation is called Schrödinger's wave equation including the time:

$$
\begin{aligned}
& -\frac{h^{2}}{8 \pi^{2} m}\left\{\frac{\partial^{2} \Psi(x, y, z, t)}{\partial x^{2}}+\frac{\partial^{2} \Psi(x, y, z, t)}{\partial y^{2}}+\frac{\partial^{2} \Psi(x, y, z, t)}{\partial z^{2}}\right\} \\
& +V(x, y, z) \Psi(x, y, z, t)=-\frac{h}{2 \pi i} \frac{\partial \Psi(x, y, z, t)}{\partial t}
\end{aligned}
$$

Here, $h$ is Planck's constant (already defined), $m$ is the mass of the particle, $V(x, y, z)$ is the potential (energy) of the conservative field (which is independent of time for conservative systems), and $i$ is the complex quantity $\sqrt{ }(-1)$. A more compact form of the equation is:

$$
-\frac{h^{2}}{8 \pi^{2} m} \nabla^{2} \Psi(x, y, z, t)+V(x, y, z) \Psi(x, y, z, t)=-\frac{h}{2 \pi i} \frac{\partial \Psi(x, y, z, t)}{\partial t}
$$

, where the Laplacian operator $\nabla^{2}$ in Cartesian coordinates is used. 


\section{Quantum Operator Associated With the Hamiltonian:}

The Hamiltonian of a single particle in a conservative field is the sum of its potential energy $(V)$ and kinetic energy $(T)$, which is furthermore a constant of the motion (from Hamiltonian dynamics). In Cartesian coordinates, this statement can be mathematically expressed as:

$$
H\left(p_{x}, p_{y}, p_{z}, x, y, z\right)=T\left(p_{x}, p_{y}, p_{z}\right)+V(x, y, z)=W, \text { a constant }
$$

Here, $p_{k}$ is the linear momentum of the particle along the $k^{\text {th }}$ Cartesian axis. Using the expression for kinetic energy of a particle, equation (D8) can be written as:

$$
H\left(p_{x}, p_{y}, p_{z}, x, y, z\right)=\frac{1}{2 m}\left(p_{x}^{2}+p_{y}^{2}+p_{z}^{2}\right)+V(x, y, z)=W
$$

Comparing equation (D9) with equation (D6), it is seen that equation (D9) can be converted into equation (D6) by the following steps:

a) Replace every $p_{k}$ in equation (D9) with $\frac{h}{2 \pi i} \frac{\partial}{\partial k}, k=x, y, z$. This is an example of a differential operator.

b) Replace $W$ in equation (D9) with $-\frac{h}{2 \pi i} \frac{\partial}{\partial t}$. This is another example of a differential operator.

c) Use the resulting differential operator equation and operate on the probability amplitude $\Psi$.

The following property of differential operators is used in the steps: 


$$
\left[C \frac{\partial}{\partial k}\right]^{n}=C^{n} \frac{\partial^{n}}{\partial k^{n}}
$$

Also, remember that $i^{2}=-1$.

Defining $H_{o}$ (subscript ' $\mathrm{o}$ ') as the quantum operator associated with the Hamiltonian (obtained by going through the afore-mentioned steps), equation (D6) can be written as:

$$
\begin{aligned}
& H_{o} \Psi(x, y, z, t)=H\left(\frac{h}{2 \pi i} \frac{\partial}{\partial x}, \frac{h}{2 \pi i} \frac{\partial}{\partial y}, \frac{h}{2 \pi i} \frac{\partial}{\partial z}, x, y, z\right) \Psi(x, y, z, t) \\
& =-\frac{h}{2 \pi i} \frac{\partial}{\partial t} \Psi(x, y, z, t)
\end{aligned}
$$

, where:

$$
H_{o}=H\left(\frac{h}{2 \pi i} \frac{\partial}{\partial x}, \frac{h}{2 \pi i} \frac{\partial}{\partial y}, \frac{h}{2 \pi i} \frac{\partial}{\partial z}, x, y, z\right)
$$

Hence it is found that the Hamiltonian operator in quantum mechanics is obtained from the classical Hamiltonian expression by replacing the coordinates and momenta by their respective quantum operators, given below:

$$
\begin{aligned}
& x_{o}=x \\
& y_{o}=y \\
& z_{o}=z \\
& p_{x o}=\frac{h}{2 \pi i} \frac{\partial}{\partial x} \\
& p_{y o}=\frac{h}{2 \pi i} \frac{\partial}{\partial y} \\
& p_{z o}=\frac{h}{2 \pi i} \frac{\partial}{\partial z}
\end{aligned}
$$




\section{Expectation (Average) Values of Observable Quantities:}

The average value or expectation value of any observable quantity $A\left(p_{x}, p_{y}, p_{z}, x, y, z\right)$ at a fixed time $t_{0}$ is defined by the following equation:

$$
\bar{A}=\iiint \Psi^{*}\left(x, y, z, t_{0}\right) A_{o} \Psi\left(x, y, z, t_{0}\right) d x d y d z
$$

, where $A_{o}$ is the quantum operator associated with the observable quantity $A\left(p_{x}, p_{y}, p_{z}, x, y, z\right)$ and is obtained using an equation analogous to equation (D12):

$$
A_{o}=A\left(\frac{h}{2 \pi i} \frac{\partial}{\partial x}, \frac{h}{2 \pi i} \frac{\partial}{\partial y}, \frac{h}{2 \pi i} \frac{\partial}{\partial z}, x, y, z\right)
$$

Note that the order of operations in the integrand of equation (D14) must not be changed. Also note that $A_{o}$ must be first recast as a Hermitian operator before using it in equation (D14). A Hermitian operator, $F_{o}$, has the following property (which is extremely important in quantum mechanics):

$$
\iiint u_{1}^{*}(x, y, z) F_{o} u_{2}(x, y, z) d x d y d z=\iiint u_{2}(x, y, z)\left[F_{o} u_{1}(x, y, z)\right]^{*} d x d y d z
$$

, where $u_{1}$ and $u_{2}$ are any operands on which the operator can operate.

The product of two Hermitian operators $F_{o}$ and $G_{o}$ (which is $F_{o} G_{o}$ ) is itself Hermitian only if $F_{o}$ and $G_{o}$ are Hermitian and commute (i.e. $F_{o} G_{o} u=G_{o} F_{o} u$ ). However, the symmetrized product, $\left(F_{o} G_{o}+G_{o} F_{o}\right) / 2$, is always Hermitian if $F_{o}$ and $G_{o}$ are themselves Hermitian. It is this symmetrized product that is used in equation (D14). Note that the Hamiltonian quantum operator is already Hermitian, and therefore does not need to be recast (symmetrized) as above.

As an example, what is the operator associated with the observable quantity $A\left(p_{x}, p_{y}, p_{z}, x, y, z\right)=x p_{x}$ ? Following equation (D15), or by multiplying the quantum operators for $x$ and $p_{x}$ from equations (D13): 


$$
A_{o}=x_{o} p_{x o}=x \frac{h}{2 \pi i} \frac{\partial}{\partial x}
$$

However, by using $A_{o}$ in equation (D16), it is found that LHS $\neq$ RHS, i.e. $A_{o}$ is not Hermitian. Hence symmetrizing the product, the correct quantum operator associated with $A_{o}$ is:

$$
A_{o}=\frac{x_{o} p_{x o}+p_{x o} x_{o}}{2}=\frac{1}{2}\left[x\left(\frac{h}{2 \pi i} \frac{\partial}{\partial x}\right)+\frac{h}{2 \pi i} \frac{\partial}{\partial x}(x)\right]
$$

Note the difference between the two terms in equation (D18): the first term takes the partial derivative of an operand with respect to $x$ and then multiplies it by $x$, whereas the second term multiplies the operand by $x$ first, and then takes the partial derivative of this product.

\section{D6. Separation of Schrödinger's Wave Equation}

Recall Schrödinger's wave equation including the time:

$$
-\frac{h^{2}}{8 \pi^{2} m} \nabla^{2} \Psi(x, y, z, t)+V(x, y, z) \Psi(x, y, z, t)=-\frac{h}{2 \pi i} \frac{\partial \Psi(x, y, z, t)}{\partial t}
$$

This equation can be separated into one differential equation in the Cartesian coordinates alone and another equation in the time alone. If the wave function is assumed to have the following form:

$$
\Psi(x, y, z, t)=\psi(x, y, z) \varphi(t)
$$


, and is substituted into equation (D19), then the equation separates into the two required differential equations:

$$
\begin{gathered}
\frac{d \varphi(t)}{d t}=-\frac{2 \pi i}{h} W \varphi(t) \\
-\frac{h^{2}}{8 \pi^{2} m} \nabla^{2} \psi(x, y, z)+V(x, y, z) \psi(x, y, z)=W \psi(x, y, z)
\end{gathered}
$$

In equations (D21) and (D22), $\mathrm{W}$ is the separation constant. Equation (D22) is customarily written as:

$$
\nabla^{2} \psi(x, y, z)+\frac{8 \pi^{2} m}{h^{2}}\{W-V(x, y, z)\} \psi(x, y, z)=0
$$

Equation (D23) is called Schrödinger's wave equation independent of time. It is found that equation (D23) possesses various satisfactory solutions, corresponding to certain values of the separation constant $W$. Let these values of $W$ be indicated by attaching the subscript $n$, and similarly let the amplitude function in the coordinates corresponding to $W_{n}$ be denoted as $\psi_{n}(x, y, z)$. For a particular value of $n$, the corresponding equation for $\varphi(t)$ (equation (D21)) can be immediately integrated to give:

$$
\varphi_{n}(t)=e^{-2 \pi i \frac{W_{n}}{h} t}
$$

Hence, the general solution of equation (D19) is:

$$
\Psi(x, y, z, t)=\sum_{n} a_{n} \Psi_{n}(x, y, z, t)=\sum_{n} a_{n} \psi_{n}(x, y, z) e^{-2 \pi i \frac{W_{n}}{h}}
$$

Hence all that is needed to be done is to solve equation (D23) for each system and use the solutions in equation (D25) to get the general probability amplitude function for the 
system. It can be proved that the separation constant $W$ corresponds to the energy levels of the system.

\section{D7. Stationary States}

The probability density function for the amplitude given by equation (D25) is (using equation (D3)):

$$
\begin{aligned}
& P(x, y, z, t)=\Psi^{*}(x, y, z, t) \Psi(x, y, z, t) \\
& =\sum_{n} a_{n}^{*} a_{n} \psi_{n}^{*} \psi_{n}+\sum_{m} \sum_{n} a_{m}^{*} a_{n} \psi_{m}{ }^{*} \psi_{n} e^{2 \pi t \frac{\left(W_{m}-W_{n}\right)_{t}}{h}}
\end{aligned}
$$

, where the prime on one the double-summation indicates that only terms with $m \neq n$ are included. From equation (D26), it is seen that $P$ is generally a function of time. For $P$ to be independent of time, the double summation must disappear. This is only possible if only one of the coefficients, $a_{n}$, is non-zero and all other coefficients are zero. In this case, the general solution (D25) will contain only one term (with $n=n$ ', say):

$$
\Psi(x, y, z, t)=\Psi_{n^{\prime}}(x, y, z, t)=\psi_{n^{\prime}}(x, y, z) e^{-2 \pi i \frac{W_{n^{\prime}}}{h}}
$$

In equation (D27), the constant $a_{n}$, has been absorbed into $\psi_{n}$. For such a state (i.e. with a single term in the solution), $P(x, y, z, t)$ is independent of time, i.e. $P=P(x, y, z)$, and the state is called a stationary state.

\section{D8. The One-Dimensional Quantum Harmonic Oscillator}

Consider a particle of mass $m$ under the influence of a Hooke's law force field along the $\mathrm{X}$-direction. If $v_{0}$ is the fundamental frequency, the potential function is $V=2 \pi^{2} m v_{0}^{2} x^{2}$. Hence the Schrödinger's wave equation independent of time (equation (D23)) becomes: 


$$
\frac{d^{2} \psi}{d x^{2}}+\frac{8 \pi^{2} m}{h^{2}}\left(W-2 \pi^{2} m v_{0}^{2} x^{2}\right) \psi=0
$$

This equation can be solved using series methods. Without going into details of the solution procedure, the following points are noted about the series solution.

1. The infinite series solution is found to be divergent. However, the probability amplitude must be integrated to give a value of 1 over the entire $x$ axis. The only way this is found to be possible is to break off the infinite series after a finite number of terms.

2. The mathematical expression for the condition in point 1 (i.e. for the condition that the series solution must be finite and not infinite) leads to quantization of energy levels, given by:

$$
W_{n}=\left(n+\frac{1}{2}\right) h v_{0}, \mathrm{n}=0,1,2, \ldots
$$

3. Unlike the old quantum theory where quantization was a required postulate, quantization follows naturally in the new quantum theory from the requirement that the solution must be physically permissible (which is a natural, unquestionable requirement in almost every branch of science). This is seen from points 1 and 2 . Hence the new quantum theory (referred to simply as quantum mechanics) requires no quantization condition like the older Wilson-Sommerfeld quantization rule.

\section{D9. The Particle in a One-Dimensional Box}

Consider a particle of mass $m$ in a one-dimensional box. A box refers to a square potential well, i.e. a potential function of the following form: 


$$
V=\left\{\begin{array}{l}
\text { infinite, }-\infty<x<0 \\
\text { constant, } 0 \leq x \leq a \\
\text { infinite, } a<x<\infty
\end{array}\right.
$$

Since potential energy is a relative concept, the constant potential in $[0, a]$ can be arbitrarily taken as zero. Hence:

$$
V=\left\{\begin{array}{c}
\infty,-\infty<x<0 \\
0,0 \leq x \leq a \\
\infty, a<x<\infty
\end{array}\right.
$$

Outside the box, the coefficient of $\psi$ in equation (D23) becomes infinite. Hence, the only solution to the wave equation that still satisfies equation (D23) is $\psi=0$. Therefore, outside the potential box:

$$
\psi(x)=0
$$

Inside the potential box, equation (D23) becomes (for $V=0$ ):

$$
\frac{d^{2} \psi}{d x^{2}}+\frac{8 \pi^{2} m}{h^{2}} W \psi=0
$$

The solution of equation (D33) is very simple, and is:

$$
\psi(x)=A \sin \left\{\left(\sqrt{\frac{8 \pi^{2} m}{h^{2}} W}\right) x\right\}
$$

This function must go to zero at $x=0$ and $x=a$ to ensure continuity with the outside solution (equation (D32)). This is again a mathematical expression for the solution to be physically permissible. At $x=0$, equation (D34) is identically satisfied (this is also the 
reason why a cosine expression of the form of equation (D34) cannot be a solution, and only a sine expression can). At $x=a$ :

$$
\sin \left\{\left(\sqrt{\frac{8 \pi^{2} m}{h^{2}} W}\right) a\right\}=0
$$

This is possible if:

$$
\left(\sqrt{\frac{8 \pi^{2} m}{h^{2}} W}\right) a=n_{x} \pi, n_{x}=1,2,3, \ldots
$$

Solving for $W$ yields:

$$
W_{n_{x}}=\frac{n_{x}{ }^{2} h^{2}}{8 m a^{2}}
$$

, i.e. the energy of the particle is quantized. Again, quantization arises directly from the requirement that the solution be physically permissible.

By substituting (D37) into (D32), the stationary state of a particle in a onedimensional box is:

$$
\psi_{n_{x}}(x)=A \sin \frac{n_{x} \pi x}{a}
$$

The constant $A$ is chosen to satisfy the normalization condition. Hence:

$$
\int_{0}^{a} \psi_{n_{x}} *(x) \psi_{n_{x}}(x) d x=1
$$

, since the range of a non-trivial solution is $[0, a]$. Substituting and solving for $A$ yields: 


$$
A=\sqrt{\frac{2}{a}}
$$

The final stationary state is:

$$
\psi_{n_{x}}(x)=\sqrt{\frac{2}{a}} \sin \frac{n_{x} \pi x}{a}
$$

The calculation can be extended to three-dimensions using the technique of separation of variables.

\section{D10. The Hydrogen Atom}

The first major application of Schrödinger's wave mechanics was to the Hydrogen atom. From Rutherford's discovery of the structure of an atom, the Hydrogen atom can be considered as having a nucleus (idealized as a point mass) of unit positive charge and mass $m_{l}$, located at the point $\left(x_{1}, y_{1}, z_{1}\right)$, and an electron of unit negative charge and mass $m_{2}$, located at the point $\left(x_{2}, y_{2}, z_{2}\right)$. The wave equation of the system is:

$$
\frac{1}{m_{1}}\left(\frac{\partial^{2} \psi_{T}}{\partial x_{1}{ }^{2}}+\frac{\partial^{2} \psi_{T}}{\partial y_{1}{ }^{2}}+\frac{\partial^{2} \psi_{T}}{\partial z_{1}^{2}}\right)+\frac{1}{m_{2}}\left(\frac{\partial^{2} \psi_{T}}{\partial x_{2}^{2}}+\frac{\partial^{2} \psi_{T}}{\partial y_{2}^{2}}+\frac{\partial^{2} \psi_{T}}{\partial z_{2}^{2}}\right)+\frac{8 \pi^{2}}{h^{2}}\left(W_{T}-V\right) \psi_{T}=0
$$

, where:

$$
V=\frac{Z e^{2}}{\sqrt{\left(x_{2}-x_{1}\right)^{2}+\left(y_{2}-y_{1}\right)^{2}+\left(z_{2}-z_{1}\right)^{2}}}
$$

is the Coulomb electrostatic potential between the nucleus and electron, and the subscript $T$ (signifying total) indicates that $\psi$ and $W$ refer to the complete system, with six 
coordinates. $Z$ is the number of protons in the nucleus. Hence this model applies not only to the Hydrogen atom (which has one proton in the nucleus, i.e. $Z=1$ ), but to any atom with $Z$ protons in the nucleus, but only one electron. This last point is important, since the following results cannot be applied directly to atoms with more than one electron.

Just as in orbital mechanics, equation (D42) can be separated into two equations, one describing the translational motion of the centre-of-mass of the nucleus and proton, and another describing the motion of the electron relative to the nucleus. The former equation is of little interest: it is the motion of the electron relative to the nucleus that is of most interest. This latter equation is best solved when written in polar coordinates (actually, the process of separation is carried out using a transformation to six new coordinates: three Cartesian coordinates for the location of the centre-of-mass of the system, and three polar coordinates for the relative position of the electron with respect to the nucleus; hence the equation of relative motion is already in polar coordinates, once the transformation and separation are effected).

The polar equation of relative motion can be solved using the method of separation of variables. Details of the long and tedious solution procedure are deferred to books on the subject. Three of the four quantum numbers popular in quantum chemistry appear during the solution procedure as a consequence of imposing the requirement that the solution be physically permissible. These are: the principal or total quantum number $(n)$; the azimuthal quantum number $(l)$; and the magnetic quantum number $(m)$. The remaining quantum number, the spin quantum number $(s)$, will be discussed shortly. The ranges of the three resulting quantum numbers are:

$$
\begin{aligned}
& n=1,2,3, \ldots \\
& l=0,1,2, \ldots, n-1 \\
& m=-l,-l+1,-l+2, \ldots-1,0,1, \ldots l-2, l-1, l
\end{aligned}
$$

A particular combination of quantum numbers is an orbital (recall from fundamental chemistry: $1 s, 2 s, 2 p_{x}, 2 p_{y}, 2 p_{z}$, etc.) where the electron is likely to be found. The energy of relative motion of the system is found to be quantized, depending only on the principal quantum number $(n)$. It is given by: 


$$
W_{n}=-\frac{2 \pi^{2} \mu Z^{2} e^{4}}{n^{2} h^{2}}
$$

, where:

$$
\mu=\frac{m_{1} m_{2}}{m_{1}+m_{2}}
$$

is the reduced mass of the system (just as in orbital mechanics).

\section{D11. Orthonormality of Wave Functions}

Recall the normalization condition of a wave function:

$$
\iiint \Psi^{*}\left(x, y, z, t_{0}\right) \Psi\left(x, y, z, t_{0}\right) d x d y d z=1
$$

The normalization condition is valid at a fixed time $t_{0}$. If the wave function is not normalized for some reason (i.e. if the wave function does not satisfy equation (D47)), then it can be normalized by multiplying by an appropriate real constant. That constant is given by the following equation:

$$
A=\sqrt{\frac{1}{\iiint \Psi^{*}\left(x, y, z, t_{0}\right) \Psi\left(x, y, z, t_{0}\right) d x d y d z}}
$$

So any wave function, in principle, can be normalized using the above equation. Whether the integral is easy to evaluate is another matter.

Now, recall the property of a Hermitian operator $F_{o}$ : 


$$
\iiint u_{1}^{*}(x, y, z) F_{o} u_{2}(x, y, z) d x d y d z=\iiint u_{2}(x, y, z)\left[F_{o} u_{1}(x, y, z)\right]^{*} d x d y d z
$$

The Hamiltonian operator satisfies equation (D49), and hence is Hermitian. Using this fact, the following important properties of the spatial wave functions (solutions to Schrödinger's wave equation independent of time, i.e. to:

$$
\left[-\frac{h^{2}}{8 \pi^{2} m} \nabla^{2}+V(x, y, z)\right] \psi_{n}(x, y, z)=W_{n} \psi(x, y, z)
$$

), can be proved. Let $\psi_{m}$ and $\psi_{n}$ (called eigen-functions of equation (D50)) be any two solutions to equation (D50), corresponding to the energy values $W_{m}$ and $W_{n}$ (called eigenvalues of equation (D50)) respectively. Three possibilities arise:

1. $m=n$ :

In this case, it can be proved that the energy eigen-value corresponding to state $m$ is real.

2. $\quad m \neq n$ and $W_{m} \neq W_{n}$ :

In this case, it can be proved that the energy eigen-functions $\psi_{m}$ and $\psi_{n}$ are orthogonal to each other:

$$
\iiint \psi_{m}{ }^{*} \psi_{n} d x d y d z=0
$$

3. $\quad m \neq n$ and $W_{m}=W_{n}$ :

In this case, the states $\mathrm{m}$ and $\mathrm{n}$ are called degenerate states. They do not necessarily satisfy the orthogonality condition of equation (D51), but can be made into orthogonal functions by mutual linear combination. These new orthogonal functions are also solutions to the wave equation for the same eigen-value $W_{m}$ (= $W_{n}$ ) because the Schrödinger's wave equation independent of time is a linear differential equation. 
Hence, in general, the wave function solutions to equation (D50) are (or can be made to form) an orthonormal (orthogonal and normalized) set, satisfying:

$$
\begin{aligned}
& \iiint \psi_{m}{ }^{*} \psi_{n} d x d y d z=1, \mathrm{~m}=\mathrm{n} \\
& \iiint \psi_{m}{ }^{*} \psi_{n} d x d y d z=0, \mathrm{~m} \neq \mathrm{n}
\end{aligned}
$$

If all solutions to the wave equation (D50) are found, then the orthonormal set is said to be complete.

\section{D12. Spin Quantum Number}

Three quantum numbers were introduced for the hydrogen atom: the principal quantum number, the azimuthal quantum number and the magnetic quantum number. These names are retained for all other multi-electron atoms as well. In addition to these three quantum numbers, there is a quantum number that is associated with electron spin, a fundamental property of the electron. This quantum number is called the spin quantum number $(s)$ and is quantized to have a value of $1 / 2$ or $-1 / 2$. These numbers are called the spin eigen-values, and are associated with a spin eigen-function equation (which involves a spin operator).

\section{D13. Pauli Exclusion Principle and Antisymmetric Wave Functions}

Each electron in a multi-electron atom has a corresponding wave equation, which includes inter-electron interactions. The wave functions which are solutions to these wave equations are found to possess an important and far-reaching property, the proof of which can be found in any good quantum mechanics textbook. The property has been called the 
Pauli exclusion principle. It states that no two electrons in an atom can have the same values for all four quantum numbers (principal, azimuthal, magnetic and spin). This statement is the final outcome of an important observation of nature: all wavefunctions in nature are antisymmetric with respect to interchange of two electrons. This is illustrated with the following example.

Consider a multi-electron atom, in which the inter-electron interactions are completely neglected. In this case, the wave equation for the atom is separable into a wave equation for each electron, which involves coordinates only of that electron. This is simply the wave equation for a hydrogen-like ion, for which the solutions are exactly known. Because of the separation of the solution, the solution for the original wave equation is simply the product of solutions of the individual (separated) wave equations. For instance, take the helium atom, which has two electrons. Neglecting inter-electron interaction completely, if $\psi_{1}$ and $\psi_{2}$ are the solutions to the separated (spatial) wave equations for the electrons, then the solution to the helium atom, neglecting the interelectron interaction, is:

$$
\psi_{H e}=\psi_{1}\left(\vec{r}_{1}\right) \psi_{2}\left(\vec{r}_{2}\right)
$$

Here, the vectors are the position vectors of electrons 1 and 2. Now, the subscripts 1 and 2 are mere labels for the electrons, and hence can be interchanged without affecting the meaning of the wave function in any way. Doing this, we find:

$$
\psi_{H e, P}=\psi_{1}\left(\vec{r}_{2}\right) \psi_{2}\left(\vec{r}_{1}\right)
$$

The subscript $P$ indicates that the electron coordinates have been permuted. Now equations (D53) and (D54) are identical, since it is only the label on an electron that we are changing. In particular, the sign of the wave function is unchanged with the interchange of the 2 electrons. Such a wave function that does not change sign with a single exchange of 2 electrons, the wave function in equation (D53) is a symmetric wave function. If the sign changes, then the wave function is an antisymmetric wave function. All wave functions in nature are found to be antisymmetric. 


\section{D14. Antisymmetrization: The Slater Determinant}

The correct way to form the composite wave function from the individual electron wave functions for $n$ electrons (neglecting inter-electron interactions) is to use the Slater determinant, defined as:

$$
\Psi\left(\vec{x}_{1}, \vec{x}_{2}, \ldots, \vec{x}_{n}\right)=\frac{1}{\sqrt{n !}}\left|\begin{array}{cccc}
\Psi_{1}\left(\vec{x}_{1}\right) & \Psi_{1}\left(\vec{x}_{2}\right) & \ldots & \Psi_{1}\left(\vec{x}_{n}\right) \\
\Psi_{2}\left(\vec{x}_{1}\right) & \Psi_{2}\left(\vec{x}_{2}\right) & \ldots & \Psi_{2}\left(\vec{x}_{n}\right) \\
\ldots & \ldots & \ldots & \ldots \\
\Psi_{n}\left(\vec{x}_{1}\right) & \Psi_{n}\left(\vec{x}_{2}\right) & \ldots & \Psi_{n}\left(\vec{x}_{n}\right)
\end{array}\right|
$$

The $x$ vectors refer to all the spatial-spin coordinates for each electron, and should not be confused with the Cartesian $x$. A spin coordinate does not have any unit, but is included to indicate that the complete wave function includes the Cartesian components $x, y$ and $z$ and a spin component to indicate the spin of each electron $(1 / 2$ or $-1 / 2)$. The $\frac{1}{\sqrt{n !}}$ factor normalizes the resulting wave function, as is required for any wave function.

\section{D15. Expansion in terms of a Complete Orthonormal Set}

One of the most useful mathematical tools is the Fourier series, which says that any periodic, well behaved function can be expanded exactly as an infinite series of sines and cosines. An infinite sequence of sines and cosines is a complete, orthonormal set. Hence the Fourier series is one type of expansion of a function in terms of a complete orthonormal set.

This fact has profound implications for the quantum mechanical solution of complex systems, especially multi-electron atoms. The hydrogen atom and hydrogen-like 
ions (ions with only one electron) have been solved exactly, and the solutions form a complete orthonormal set. Hence any wave function of any system can be exactly represented by an infinite series of the wave functions of a hydrogen atom. This is the single, important principle that has allowed practical solutions of any system more complex than the Hydrogen atom, and is the foundation for almost all numerical procedures in quantum mechanics.

For a multi-electron system, this expansion results in an infinite series of Slater determinants. Because there are an infinite number of wave functions that solve a single electron system (corresponding to an infinite number of principal quantum number), each Slater determinant places the $n$ electrons in $n$ of these single-electron wave functions, giving:

$$
\Psi\left(\vec{x}_{1}, \vec{x}_{2}, \ldots, \vec{x}_{n}\right)=\sum_{K=1}^{\infty} D_{K} \Phi_{K}
$$

The Slater determinants have been renamed as $\Phi_{\mathrm{K}}$ (each of which is given by the RHS of equation (D55)), and $D_{K}$ are constant multiplicative factors. They are constant because the expansion is already in terms of wave functions that have similar properties to the total wave function $\Psi$.

\section{D16. Hartree-Fock Self-Consistent-Field Method}

The Hartree-Fock (HF) Self-Consistent-Field (SCF) [32] method attempts to optimize the single determinant approximation in equation (D56):

$$
\Psi\left(\vec{x}_{1}, \vec{x}_{2}, \ldots, \vec{x}_{n}\right)=\Phi_{1}
$$

$D_{1}$ is taken as one initially since only a single determinant is involved. A suitable normalization constant can be computed later. The optimization of the member orbitals of $\Phi_{1}$ is done by minimizing the system energy, given by: 


$$
W=\frac{\int \Psi^{*} H_{o} \Psi d \tau}{\int \Psi^{*} \Psi d \tau}
$$

, where $H_{o}$ is the Hamiltonian operator (this time including the inter-electron interactions) and $d \tau$ includes all position variables and the spin variable. This procedure is based on the variational principle of quantum mechanics, which states that a value of energy obtained from an approximate wave function is always greater than the actual energy value.

Each component orbital of the determinant $\Phi_{K}$ is in turn approximated as linear combinations of single-electron atomic orbitals, simply called Linear Combination of Atomic Orbitals (LCAO). Hence:

$$
\Psi_{i}=\sum_{r=1}^{m} \varphi_{r} C_{r i}
$$

for each $\Psi_{\mathrm{i}}$ in the RHS of equation (D55). The coefficients $C_{r i}$ are ultimately the constants to be iteratively determined to optimize the wave function. Complete details of the derivation can be found in [32], and is not repeated here due to the sheer amount of material.

The atomic orbitals $\varphi_{r}$ are called the basis functions for the HF calculation, and these atomic orbitals are once again expanded in terms of simple orbital-like functions called primitive basis functions:

$$
\varphi_{j}=\sum_{s=1}^{l} \phi_{s, \text { primitive }} B_{s j}
$$

This expansion has been calculated for various atoms and is available in the form of sets called basis sets. These are briefly described next. 


\section{D17. Slater Type Orbitals, Gaussian Type Orbitals and Basis Sets}

The basis functions, $\varphi_{r}$, were originally hydrogen-like radial wave functions of the form:

$$
\varphi_{r}=A \exp (-B r)
$$

This type of atomic orbital wave function is called a Slater Type Orbital (STO). It was soon found out that numerically integrating STOs was computationally very expensive. Hence today, these STOs are expanded in terms of primitive basis functions (as in equation (D60)), each of which is of the following form:

$$
\varphi_{s, \text { primitive }}=A \exp \left(-B r^{2}\right)
$$

This is a Gaussian, and hence is called a Gaussian Type Orbital (GTO). This equation (with the $r$ being squared) is far easier to numerically integrate with respect to individual Cartesian coordinates $\left(\right.$ since $\exp \left(-r^{2}\right)=\exp \left(-x^{2}\right) \exp \left(-y^{2}\right) \exp \left(-z^{2}\right)$, while $\exp (-r)$ cannot be separated into separate functions of the Cartesian coordinates as easily). This makes evaluating the variation of the energy expression (equation (D58)) far easier than if it were done using STOs.

There are several expansions of the form of equation (D60) that are used today. These basis sets are usually named based on the number of primitives used for the expansion.

\section{D18. Hartree-Fock Analysis of Copper using WinGAMESS}

The General Atomic and Molecular Electronic Structure System (GAMESS) is a free computational quantum chemistry software that performs HF analysis on user-defined 
molecules and gives the user a choice of several basis sets ${ }^{\dagger \dagger}$. WinGAMESS is the Windows $^{\mathrm{TM}}$ version of GAMESS.

The goal is to determine the electron density of copper. The input file for WinGAMESS is given below.

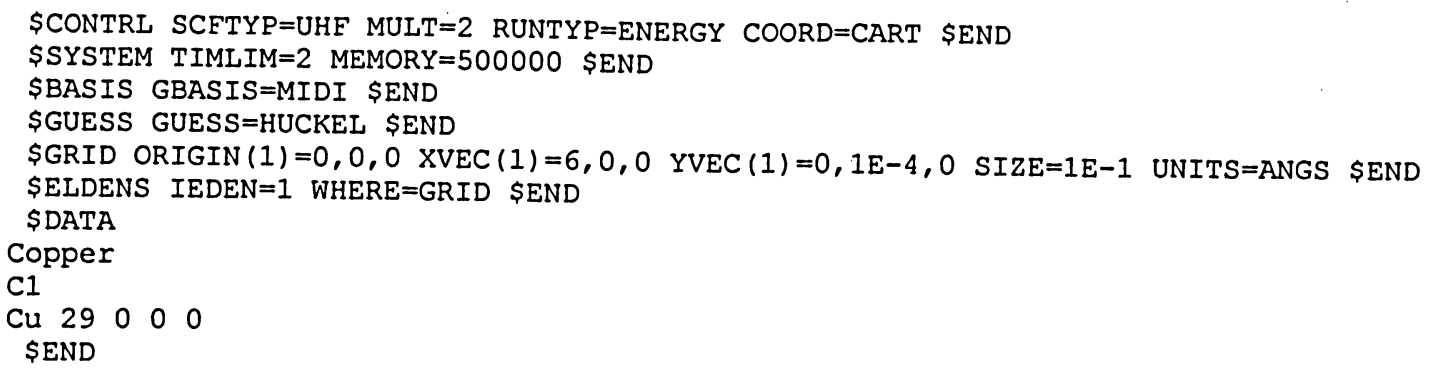

UHF refers to Unrestricted Hartree Fock and is the most general HF run available. The spin multiplicity of copper is 2 , i.e. copper has 1 unpaired electron (MULT=2). The copper atom is centered at $(0,0,0)$ and has 29 electrons. The basis set used is Huzinaga's MIDI basis set, which is available in WinGAMESS.

The output from WinGAMESS for the input given above is given on the following pages.

${ }^{\dagger+}$ GAMESS Home Page: http://www.msg.ameslab.gov/GAMESS/GAMESS.html 
Distributed Data Interface kickoff program.

Initiating 1 compute processes on 1 nodes to run the following command: C: \WinGAMESS/gamess.05.exe input

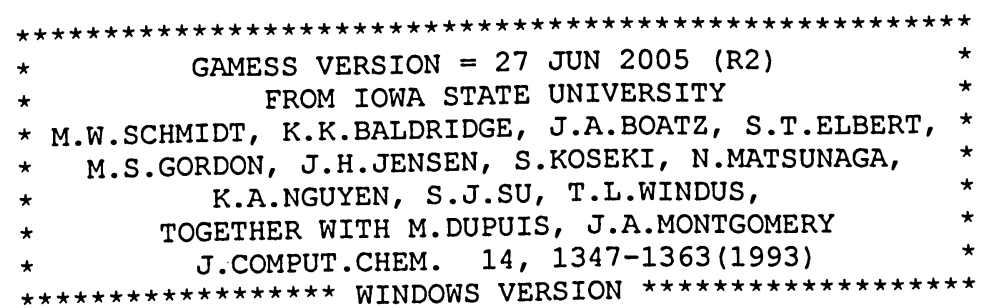

SINCE 1993, STUDENTS AND POSTDOCS WORKING AT IOWA STATE UNIVERSITY AND ALSO IN THEIR VARIOUS JOBS AFTER IEAVING ISU HAVE MADE IMPORTANT CONTRIBUTIONS TO THE CODE:

IVANA ADAMOVIC, CHRISTINE AIKENS, ROB BELL, PRADIPTA BANDYOPADHYAY, BRETT BODE, GALINA CHABAN, WEI CHEN, CHEOL HO CHOI, PAUL DAY, TIM DUDLEY, DMITRI FEDOROV, GRAHAM FLETCHER, MARK FREITAG, KURT GLAESEMANN, GRANT MERRILL, HEATHER NETZLOFE, RYAN OLSON, MIKE PAK, JIM SHOEMAKER, JIE SONG, TETSUYA TAKETSUGU, SIMON WEBB.

ADDITIONAL CODE HAS BEEN PROVIDED BY COLLABORATORS IN OTHER GROUPS: IOWA STATE UNIVERSITY: JOE IVANIC, KLAUS RUEDENBERG

UNIVERSITY OE TOKYO: KIMIHIKO HIRAO, HARUYUKI NAKANO, TAKAHITO NAKAJIMA, TAKAO TSUNEDA, MUNEAKI KAMIYA, SUSUMU YANAGISAWA, KIYOSHI YAGI

UNIVERSITY OF SOUTHERN DENMARK: FRANK JENSEN UNIVERSITY OF IOWA: VISVALDAS KAIRYS, HUI LI NATIONAL INST. OF STANDARDS AND TECHNOLOGY: WALT STEVENS, DAVID GARMER UNIVERSITY OF PISA: BENEDETTA MENNUCCI, JACOPO TOMASI UNIVERSITY OE MEMPHIS: HENRY KURTZ, PRAKASHAN KORAMBATH UNIVERSITY OF ALBERTA: MARIUSZ KLOBUKOWSKI UNIVERSITY OF NEW ENGLAND: MARK SPACKMAN MIE UNIVERSITY: HIROAKI UMEDA

MICHIGAN STATE UNIVERSITY: KAROI KOWALSKI, MARTA WLOCH, PIOTR PIECUCH UNIVERSITY OF SIIESIA: MONIKA MUSIAL, STANISLAW KUCHARSKI FACULTES UNIVERSITAIRES NOTRE-DAME DE LA PAIX: OLIVIER QUINET, BENOIT CHAMPAGNE

UNIVERSITY OF CALIFORNIA - SANTA BARBARA: BERNARD KIRTMAN

INSTITUTE FOR MOLECULAR SCIENCE: KAZUYA ISHIMURA AND SHIGERU NAGASE

UNIVERSITY OF NOTRE DAME: DAN CHIPMAN EXECUTION OE GAMESS BEGUN MON NOV " 6 18:34:59 2006

ECHO OF THE FIRST FEW INPUT CARDS -

INPUT CARD> \$CONTRI SCFTYP=UHF MULT=2 RUNTYP=ENERGY COORD=CART \$END

INPUT CARD> \$SYSTEM TIMLIM $=2$ MEMORY $=500000$ \$END

INPUT CARD> \$BASIS GBASIS=MIDI \$END

INPUT CARD> \$GUESS GUESS=HUCKEL \$END

INPUT CARD> $\$$ GRID ORIGIN $(1)=0,0,0 \operatorname{XVEC}(1)=6,0,0 \operatorname{YVEC}(1)=0,1 \mathrm{E}-4,0$ SIZE=1E-1 UNITS=ANGS \$END

INPUT CARD> \$ELDENS IEDEN=1 WHERE=GRID \$END

INPUT CARD> \$DATA

INPUT CARD>COpper

INPUT CARD $>\mathrm{C} 1$

INPUT CARD>CU 290000

INPUT CARD> \$END

INPUT CARD>

.... DONE SETTING UP THE RUN .... 
500000 WORDS OF MEMORY AVAILABLE

BASIS OPTIONS

$\begin{array}{llllll}\text { GBASIS }=\text { MIDI } & & \text { IGAUSS }= & 0 & \text { POLAR }=\text { NONE } & \\ \text { NDFUNC }= & 0 & \text { NEFUNC }= & 0 & \text { DIFFSP } & \text { F } \\ \text { NPFUNC }= & 0 & \text { DIFFS }= & \text { F } & & \end{array}$

\section{RUN TITLE}

\section{Copper}

THE POINT GROUP OF THE MOLECULE IS C1 THE ORDER OF THE PRINCIPAL AXIS IS 0

THE MOMENTS OF INERTIA ARE (AMU-ANGSTROM $\star \star 2$ )

$\begin{array}{llll}I X X & 0.000 \quad I Y Y= & 0.000 \quad I Z Z= & 0.000\end{array}$

\begin{tabular}{lcccc} 
ATOM & ATOMIC & \multicolumn{3}{c}{ COORDINATES (BOHR) } \\
CU & CHARGE & $X$ & $Y$ & $Z$ \\
& 29.0 & 0.0000000000 & 0.0000000000 & 0.0000000000
\end{tabular}

INTERNUCLEAR DISTANCES (ANGS.)

CU
$1 \mathrm{CU}$
0.0000000

*... LESS THAN 3.000

ATOMIC BASIS SET

THE CONTRACTED PRIMITIVE FUNCTIONS HAVE BEEN UNNORMALIZED THE CONTRACTED BASIS FUNCTIONS ARE NOW NORMALIZED TO UNITY
SHELI TYPE PRIMITIVE
EXPONENT
CONTRACTION COEFEICIENTS

$\mathrm{CU}$

$\begin{array}{lllrr}1 & \mathrm{~S} & 1 & 4137.5632000 & 0.063136495841 \\ 1 & \mathrm{~S} & 2 & 625.7329400 & 0.374792875314 \\ 1 & \mathrm{~S} & 3 & 136.9596900 & 0.683209455000 \\ 2 & \mathrm{~S} & 4 & 183.1051000 & -0.104686104134 \\ 2 & \mathrm{~S} & 5 & 17.1224180 & 0.703484027782 \\ 2 & \mathrm{~S} & 6 & 6.9491752 & 0.363428114352 \\ & & & & \\ 3 & \mathrm{P} & 7 & 53.6235300 & 0.092364299331 \\ 3 & \mathrm{P} & 8 & 15.3880410 & 0.615583995543 \\ 3 & \mathrm{P} & 9 & 13.9999370 & -0.223963395077 \\ & & & 2.2431225 & 0.711303284363 \\ 4 & \mathrm{~S} & 10 & 0.8531059 & 0.422701490708 \\ 4 & \mathrm{~S} & 11 & 5.8732783 & 0.259313512372 \\ 4 & \mathrm{~S} & 12 & 2.2084129 & 0.568795027137 \\ & & & 0.7864834 & 0.298710614251 \\ 5 & \mathrm{P} & 13 & 15.5864860 & 0.284218699230 \\ 5 & \mathrm{P} & 14 & & \end{array}$




\section{APPENDIX D: A BRIEF OVERVIEW OF QUANTUM MECHANICS}

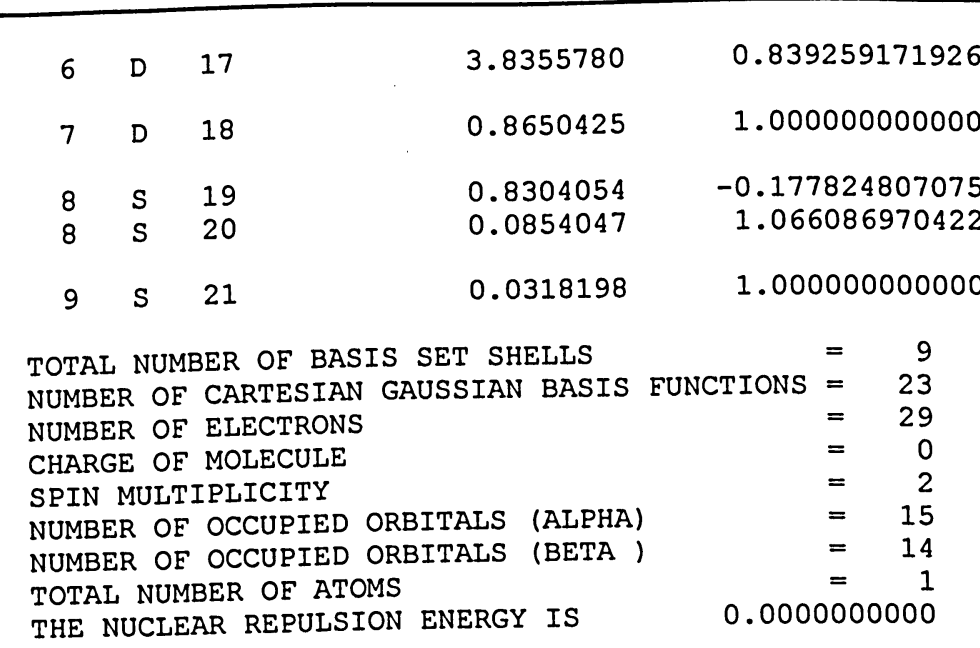

THIS MOLECULE IS RECOGNIZED AS BEING LINEAR.

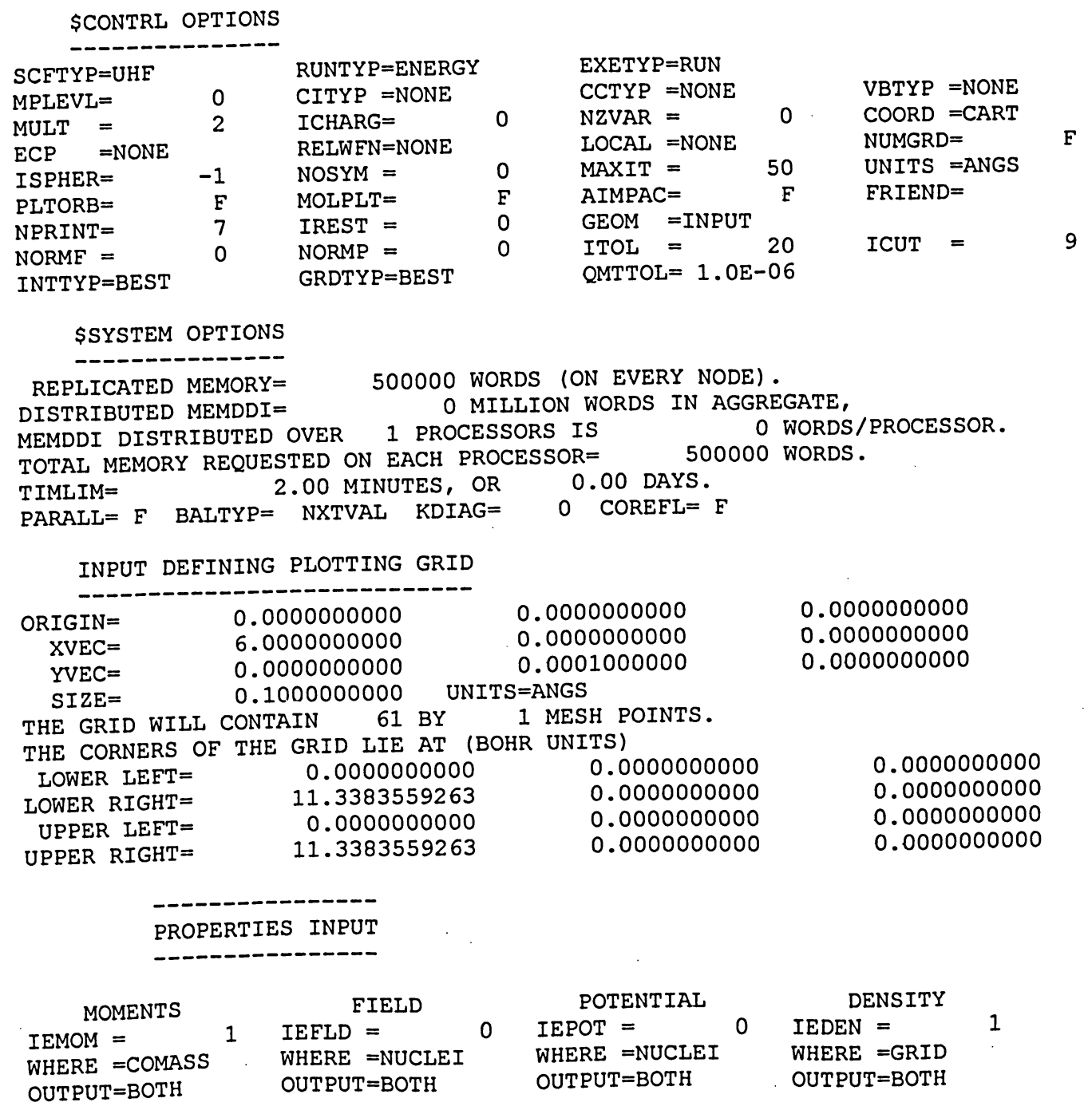




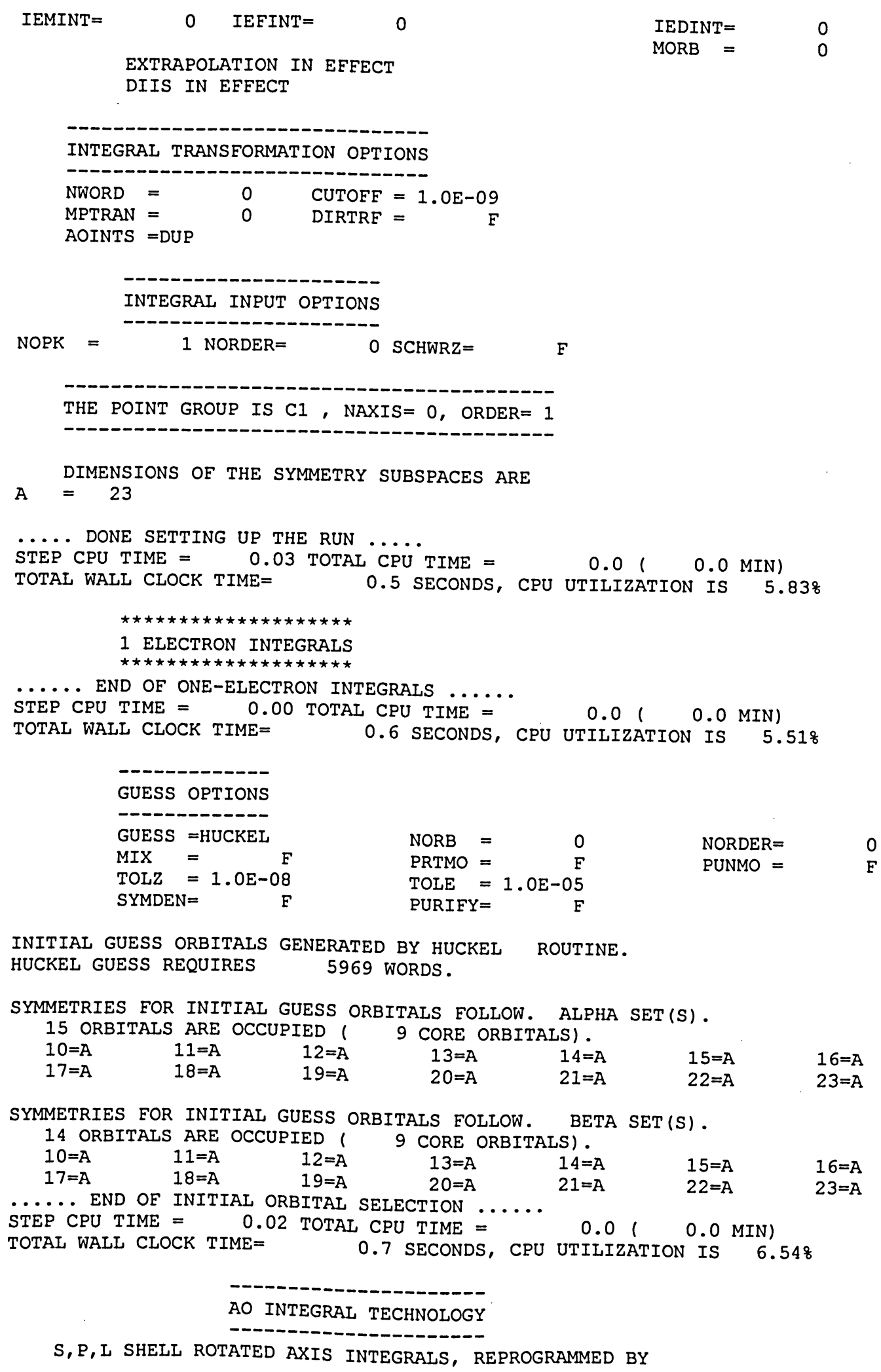

INITIAL GUESS ORBITALS GENERATED BY HUCKEL ROUTINE. HUCKEL GUESS REQUIRES 5969 WORDS.

SYMMETRIES FOR INITIAL GUESS ORBITALS FOLLOW. ALPHA SET(S).

$\begin{array}{lrrrrrr}15 & & & \\ 10=\mathrm{A} & 11=\mathrm{A} & 12=\mathrm{A} & 9 & \text { CORE ORBITALS) } & & \\ 17=\mathrm{A} & 18=\mathrm{A} & 19=\mathrm{A} & 20=\mathrm{A} & 14=\mathrm{A} & 15=\mathrm{A} & 16=\mathrm{A} \\ & & 19 & 21=\mathrm{A} & 22=\mathrm{A} & 23=\mathrm{A}\end{array}$

SYMMETRIES FOR INITIAL GUESS ORBITALS FOLLOW. BETA SET(S). 14 ORBITALS ARE OCCUPIED ( 9 CORE ORBITALS)

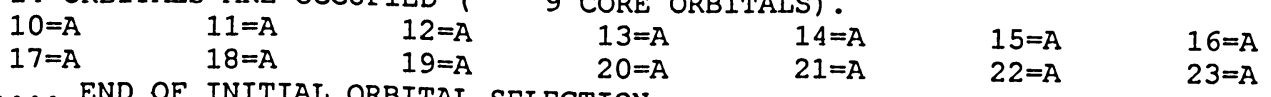

$\ldots \ldots$ END OF INITIAL ORBITAL SELECTION $\ldots \ldots$
STEP CPU TIME $=\quad 0.02$ TOTAL CPU TIME $=0.0(0.0$ MIN)

$\begin{array}{llll}\text { TOTAL WALL CLOCK TIME } & 0.7 \text { SECONDS, CPU UTILIZATION IS } 6.54 \%\end{array}$

AO INTEGRAL TECHNOLOGY

$S, P, I$ SHELI ROTATED AXIS INTEGRALS, REPROGRAMMED BY 
KAZUYA ISHIMURA (IMS) AND JOSE SIERRA (SYNSTAR).

$S, P, D, L$ SHELL ROTATED AXIS INTEGRALS PROGRAMMED BY

KAZUYA ISHIMURA (INSTITUTE FOR MOLECULAR SCIENCE).

$S, P, D, F, G$ SHELL TO TOTAL QUARTET ANGULAR MOMENTUM SUM 5 ,

ERIC PROGRAM BY GRAHAM FLETCHER (ELORET AND NASA ADVANCED

SUPERCOMPUTING DIVISION, AMES RESEARCH CENTER).

$S, P, D, F, G, L$ SHELL GENERAL RYS QUADRATURE PROGRAMMED BY

MICHEL DUPUIS (PACIFIC NORTHWEST NATIONAL LABORATORY).

2 ELECTRON INTEGRALS

THE -PK- OPTION IS OFE, THE INTEGRALS ARE NOT IN SUPERMATRIX FORM.

STORING 15000 INTEGRALS/RECORD ON DISK, USING 12 BYTES/INTEGRAL.

TWO ELECTRON INTEGRAL EVALUATION REQUIRES 60741 WORDS OF MEMORY.

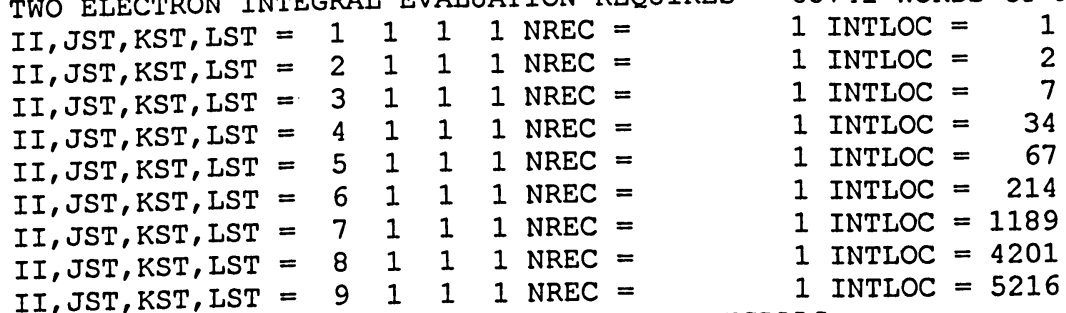

TOTAL NUMBER OF NONZERO TWO-ELECTRON INTEGRALS =

1 INTEGRAL RECORDS WERE STORED ON DISK FILE 8.

...... END OE TWO-ELECTRON INTEGRALS .....

STEP CPU TIME $=0.09$ TOTAL CPU TIME $=0.1(10.0 \mathrm{MIN})$

TOTAL WALI CLOCK TIME=

1.0 SECONDS; CPU UTILIZATION IS $14.45 \%$

UHF SCE CALCULATION

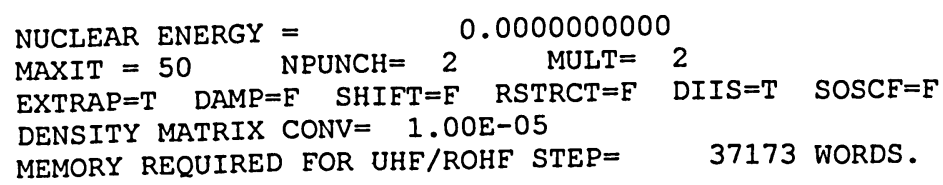

\begin{tabular}{|c|c|c|c|c|c|}
\hline TER & EX & $\begin{array}{l}\text { TOTAL ENERGY } \\
631.6367258912\end{array}$ & $\begin{array}{r}\text { E CHANGE DE } \\
-1631.6367258912\end{array}$ & $\begin{array}{l}\text { SITY CHANGE } \\
0.298663554\end{array}$ & $\begin{array}{l}\text { DIIS ERROR } \\
7.024076405\end{array}$ \\
\hline & & INITIATING & DIIS PROCEDURE & $\star \star \star \star$ & \\
\hline 2 & 1 & -1631.7938965389 & $\begin{array}{l}-0.1571706477 \\
-0.0000075096\end{array}$ & $\begin{array}{l}0.001375045 \\
0.000406995\end{array}$ & $\begin{array}{l}0.036687951 \\
0.001022709\end{array}$ \\
\hline 4 & $\begin{array}{l}2 \\
3\end{array}$ & -1631.7939045241 & -0.0000004756 & 0.000124105 & 0.000707698 \\
\hline 5 & 4 & -1631.7939047451 & -0.0000002210 & 0.000105754 & 0.000096945 \\
\hline $\begin{array}{l}6 \\
7\end{array}$ & $\begin{array}{l}5 \\
6\end{array}$ & $\begin{array}{l}-1631.7939047561 \\
-1631.7939047562\end{array}$ & $\begin{array}{l}-0.0000000110 \\
-0.0000000001\end{array}$ & $\begin{array}{l}0.000021356 \\
0.000007165\end{array}$ & $\begin{array}{l}0.000007281 \\
0.000000690\end{array}$ \\
\hline 8 & 7 & -1631.7939047562 & 0.0000000000 & 0.000002951 & 0.000000207 \\
\hline
\end{tabular}

DENSITY CONVERGED

FINAL UHF ENERGY IS $\quad-1631.7939047562$ AFTER 8 ITERATIONS

$\begin{array}{lr}\text { SPIN SZ }= & 0.500 \\ \text { S-SQUARED }= & 0.750\end{array}$


EIGENVECTORS

$-326.7337$

A

$1 \mathrm{CU} 1 \mathrm{~S}$

CU 1

CU $1 \quad X$

CU 1 Y $Y$

CU 1 Z

CU $1 \mathrm{~S}$

CU $1 \mathrm{X}$

CU 1 Y

CU 1 Z

CU $1 \mathrm{XX}$

CU 1 YY

CU 1 ZZ

CU $1 \mathrm{XY}$

CU $1 \mathrm{XZ}$

CU 1 YZ

CU $1 \mathrm{XX}$

CU 1 YY

CU $1 \mathrm{ZZ}$

CU $1 \mathrm{XY}$

CU $1 \mathrm{XZ}$

CU 1 YZ

CU $1 \mathrm{~S}$

CU $1 \mathrm{~S}$

0.979742
0.074303
0.000000
0.000000
0.000000
0.136516
0.000000
0.000000
0.000000
-0.065121
-0.065121
-0.065121
0.000000
0.000000
0.000000
-0.014303
-0.014303
-0.014303
0.000000
0.000000
0.000000
0.005443
-0.002693

\section{6}

$-4.8633$

A

0.123878

$-0.460234$

0.000000

0.000000

0.000000

1.089762

0.000000

0.000000

0.000000

0.001073

0.001073

0.001073

0.000000

0.000000

0.000000

$-0.001168$

$-0.001168$

$-0.001168$

0.000000

0.000000

0.000000

0.006013

$-0.002518$

11

$-0.2861$

A
2
-40.5852
$A$

$-0.327478$

1. 033727

0.000000

0.000000

0.000000

0.000752

0.000000

0.000000

0.000000

0.002849

0.002849

0.002849

0.000000

0.000000

0.000000

0.000717

0.000717

0.000717

0.000000

0.000000

0.000000

$-0.000742$

0.000412

$$
\begin{array}{r}
7 \\
-3.1 \\
\text { A }
\end{array}
$$

0.000000

0.000000

0.107057

$-0.375556$

0.086352

0.000000

$-0.283238$

0.993597

$-0.228460$

0.000000

0.000000

0.000000

0.000000

0.000000

0.000000

0.000000

0.000000

0.000000

0.000000

0.000000

0.000000

0.000000

0.000000

12

$-0.2861$

A

3
-35.3424
$A$
0.000000
0.000000
-0.605414
0.693577
0.339068
0.000000
-0.032945
0.037743
0.018451
0.000000
0.000000
0.000000
0.000000
0.000000
0.000000
0.000000
0.000000
0.000000
0.000000
0.000000
0.000000
0.000000
0.000000

8

$-3.17$

0.000000

0.000000

0.264846

0.006605

$-0.299621$

0.000000

$-0.700696$

$-0.017476$

0.792699

0.000000

0.000000

0.000000

0.000000

0.000000

0.000000

0.000000

0.000000

0.000000

0.000000

0.000000

0.000000

0.000000

0.000000

13

$-0.2861$

A

${ }^{4}$
-35.3424
$A$
0.000000
0.000000
0.654376
0.689653
-0.242307
0.000000
0.035610
0.037529
-0.013186
0.000000
0.000000
0.000000
0.000000
0.000000
0.000000
0.000000
0.000000
0.000000
0.000000
0.000000
0.000000
0.000000
0.000000

9 $-3.179$

0.000000

0.000000

$-0.279920$

$-0.137384$

$-0.250460$

0.000000

0.740576

0.363473

0.662636

0.000000

0.000000

0.000000

0.000000

0.000000

0.000000

0.000000

0.000000

0.000000

0.000000

0.000000

0.000000

0.000000

0.000000

14

$-0.2861$

A
5

$-35.3424$

$A$

0.000000

0.000000

0.409644

$-0.076631$

0.888179

0.000000

0.022292

$-0.004170$

0.048333

0.000000

0.000000

0.000000

0.000000

0.000000

0.000000

0.000000

0.000000

0.000000

0.000000

0.000000

0.000000

0.000000

0.000000

10 $-0.2861$ $A$

0.000000

0.000000

0.000000

0.000000

0.000000

0.000000

0.000000

0.000000

0.000000

0.000836

$-0.008879$

0.008042

$-0.020877$

$-0.046036$

0.607467

0.000827

$-0.008777$

0.007950

$-0.020638$

$-0.045508$

0.600501

0.000000

0.000000

15 $-0.2090$

A 


\begin{tabular}{|c|c|c|c|c|c|c|c|c|}
\hline 1 & $\mathrm{CU}$ & 1 & $S$ & 0.000000 & 0.000000 & 0.000000 & 0.000000 & -0.018784 \\
\hline 2 & $\mathrm{CU}$ & 1 & $\mathrm{~S}$ & 0.000000 & 0.000000 & 0.000000 & 0.000000 & 0.062439 \\
\hline 3 & $\mathrm{CU}$ & 1 & $\mathrm{x}$ & 0.000000 & 0.000000 & 0.000000 & 0.000000 & 0.000000 \\
\hline 4 & $\mathrm{CU}$ & 1 & $Y$ & 0.000000 & 0.000000 & 0.000000 & 0.000000 & 0.000000 \\
\hline 5 & $\mathrm{CU}$ & 1 & $\mathrm{z}$ & 0.000000 & 0.000000 & 0.000000 & 0.000000 & 0.000000 \\
\hline 6 & $\mathrm{CU}$ & 1 & $S$ & 0.000000 & 0.000000 & 0.000000 & 0.000000 & -0.209851 \\
\hline 7 & $\mathrm{CU}$ & 1 & $x$ & 0.000000 & 0.000000 & 0.000000 & 0.000000 & 0.000000 \\
\hline 8 & $\mathrm{CU}$ & 1 & $Y$ & 0.000000 & 0.000000 & 0.000000 & 0.000000 & 0.000000 \\
\hline 9 & $\mathrm{CU}$ & 1 & $\mathrm{z}$ & 0.000000 & 0.000000 & 0.000000 & 0.000000 & 0.000000 \\
\hline 10 & $\mathrm{CU}$ & 12 & $x \mathrm{x}$ & 0.594249 & -0.106745 & -0.076547 & 0.035784 & -0.002149 \\
\hline 11 & $\mathrm{CU}$ & 1 & YY & -0.339531 & -0.352689 & 0.341456 & 0.123794 & -0.002149 \\
\hline 12 & $\mathrm{CU}$ & 1 & $\mathrm{ZZ}$ & -0.254718 & 0.459434 & -0.264909 & -0.159578 & -0.002149 \\
\hline 13 & $\mathrm{CU}$ & 18 & $X Y$ & -0.054897 & 0.040149 & -0.218398 & 0.564720 & 0.000000 \\
\hline 14 & $\mathrm{CU}$ & 12 & $\mathrm{XZ}$ & 0.114441 & 0.371916 & 0.441076 & 0.153562 & 0.000000 \\
\hline 15 & $\mathrm{CU}$ & 1 & YZ & 0.005180 & 0.022171 & 0.031656 & 0.033627 & 0.000000 \\
\hline 16 & $\mathrm{CU}$ & 1 & $\mathrm{xx}$ & 0.587435 & -0.105521 & -0.075669 & 0.035373 & -0.000894 \\
\hline 17 & $\mathrm{CU}$ & 1 & YY & -0.335638 & -0.348645 & 0.337541 & 0.122375 & -0.000894 \\
\hline 18 & $\mathrm{CU}$ & 1 & $\mathrm{ZZ}$ & -0.251797 & 0.454166 & -0.261872 & -0.157748 & -0.000894 \\
\hline 19 & $\mathrm{CU}$ & 12 & $X Y$ & -0.054267 & 0.039688 & -0.215894 & 0.558244 & 0.000000 \\
\hline 20 & $\mathrm{CU}$ & 1 & $\mathrm{xz}$ & 0.113129 & 0.367652 & 0.436019 & 0.151802 & 0.000000 \\
\hline 21 & $\mathrm{CU}$ & 1 & $Y Z$ & 0.005121 & 0.021917 & 0.031293 & 0.033242 & 0.000000 \\
\hline 22 & $\mathrm{CU}$ & 1 & $S$ & 0.000000 & 0.000000 & 0.000000 & 0.000000 & 0.511911 \\
\hline \multirow[t]{4}{*}{23} & CU & 1 & $S$ & 0.000000 & 0.000000 & 0.000000 & 0.000000 & 0.547250 \\
\hline & & & & 16 & 17 & 18 & 19 & 20 \\
\hline & & & & 0.1477 & 1.7639 & 3.6267 & 3.6267 & 3.6267 \\
\hline & & & & $A$ & A & A & A & A \\
\hline 1 & $\mathrm{CU}$ & 1 & $S$ & -0.015440 & 0.066510 & 0.000000 & 0.000000 & 0.000000 \\
\hline 2 & $\mathrm{CU}$ & 1 & $\mathrm{~S}$ & 0.079240 & -0.243004 & 0.000000 & 0.000000 & 0.000000 \\
\hline 3 & $\mathrm{CU}$ & 1 & $\mathrm{x}$ & 0.000000 & 0.000000 & 0.000000 & 0.000000 & 0.000000 \\
\hline 4 & $\mathrm{CU}$ & 1 & $Y$ & 0.000000 & 0.000000 & 0.000000 & 0.000000 & 0.000000 \\
\hline 5 & $\mathrm{CU}$ & 1 & $\mathrm{Z}$ & 0.000000 & 0.000000 & 0.000000 & 0.000000 & 0.000000 \\
\hline 6 & $\mathrm{CU}$ & 1 & $S$ & -0.022569 & 1.327951 & 0.000000 & 0.000000 & 0.000000 \\
\hline 7 & $\mathrm{CU}$ & 1 & $\mathrm{x}$ & 0.000000 & 0.000000 & 0.000000 & 0.000000 & 0.000000 \\
\hline 8 & $\mathrm{CU}$ & 1 & $Y$ & 0.000000 & 0.000000 & 0.000000 & 0.000000 & 0.000000 \\
\hline 9 & $\mathrm{CU}$ & 1 & $\mathrm{Z}$ & 0.000000 & 0.000000 & 0.000000 & 0.000000 & 0.000000 \\
\hline 10 & $\mathrm{CU}$ & 1 & $x \bar{x}$ & -0.024281 & -0.180188 & 0.000025 & 0.035080 & 0.440160 \\
\hline 11 & $\mathrm{CU}$ & 1 & YY & -0.024281 & -0.180188 & 0.000875 & -0.000202 & -0.720316 \\
\hline 12 & $\mathrm{CU}$ & 1 & $\mathrm{ZZ}$ & -0.024281 & -0.180188 & -0.000900 & -0.034878 & 0.280156 \\
\hline 13 & $\mathrm{CU}$ & 1 & $X Y$ & 0.000000 & 0.000000 & -0.262649 & 0.025051 & 0.477366 \\
\hline 14 & $\mathrm{CU}$ & 1 & $x z$ & 0.000000 & 0.000000 & -0.015126 & -0.880735 & 0.016612 \\
\hline 15 & $\mathrm{CU}$ & 1 & $Y Z$ & 0.000000 & 0.000000 & -0.841904 & 0.008034 & -0.149912 \\
\hline 16 & $\mathrm{CU}$ & 1 & $\mathrm{xx}$ & 0.006347 & -0.738476 & -0.000026 & -0.035271 & -0.442551 \\
\hline 17 & $\mathrm{CU}$ & 1 & $Y Y$ & 0.006347 & -0.738476 & -0.000880 & 0.000203 & 0.724229 \\
\hline 18 & $\mathrm{CU}$ & 1 & $\mathrm{ZZ}$ & 0.006347 & -0.738476 & 0.000905 & 0.035068 & -0.281678 \\
\hline 19 & CU & 1 & $X Y$ & 0.000000 & 0.000000 & 0.264075 & -0.025187 & -0.479959 \\
\hline 20 & $\mathrm{CU}$ & 1 & $x z$ & 0.000000 & 0.000000 & 0.015208 & 0.8 & -0.016702 \\
\hline 21 & $\mathrm{CU}$ & 1 & $Y Z$ & 0.000000 & 0.000000 & 0.846477 & -0.008078 & 0.150726 \\
\hline 22 & $\mathrm{CU}$ & 1 & $S$ & 1.840462 & 1.159887 & 0.000000 & 0.000000 & 0.000000 \\
\hline \multirow[t]{4}{*}{23} & $\mathrm{CU}$ & 1 & $\mathrm{~S}$ & -1.828919 & -0.613398 & 0.000000 & 0.000000 & 0.000000 \\
\hline & & & & 21 & 22 & 23 & & \\
\hline & & & & 3.6267 & 3.6267 & 28.3804 & & \\
\hline & & & & & & A & & \\
\hline 1 & $\mathrm{CU}$ & 1 & $S$ & 0.000000 & 0.000000 & -0.296924 & & \\
\hline 2 & $\mathrm{CU}$ & 1 & $S$ & 0.000000 & 0.000000 & 1.317391 & & \\
\hline 3 & $\mathrm{CU}$ & 1 & $\mathrm{x}$ & 0.000000 & 0.000000 & 0.000000 & & \\
\hline 4 & $\mathrm{CU}$ & 1 & $Y$ & 0.000000 & 0.000000 & 0.000000 & & \\
\hline 5 & $\mathrm{CU}$ & 1 & $z$ & 0.000000 & 0.000000 & 0.000000 & & \\
\hline 6 & $\mathrm{CU}$ & 1 & $S$ & 0.000000 & 0.000000 & 12.268098 & & \\
\hline 7 & $\mathrm{CU}$ & 1 & $\mathrm{x}$ & 0.000000 & 0.000000 & 0.000000 & & \\
\hline 8 & $\mathrm{CU}$ & 1 & $Y$ & 0.000000 & 0.000000 & 0.000000 & & \\
\hline 9 & $\mathrm{CU}$ & 1 & $\mathrm{z}$ & 0.000000 & 0.000000 & 0.000000 & & \\
\hline
\end{tabular}




\begin{tabular}{|c|c|c|c|c|c|}
\hline 10 & $\mathrm{CU}$ & $1 \mathrm{xx}$ & 0.656550 & 0.389857 & -5.077984 \\
\hline 11 & $\mathrm{CU}$ & $1 Y Y$ & 0.174411 & -0.478269 & -5.077984 \\
\hline 12 & $\mathrm{CU}$ & $1 \mathrm{zZ}$ & -0.830961 & 0.088412 & -5.077984 \\
\hline 13 & $\mathrm{CU}$ & $1 X Y$ & 0.087004 & -0.687719 & 0.000000 \\
\hline 14 & $\mathrm{CU}$ & $1 \mathrm{XZ}$ & 0.041572 & -0.009515 & 0.000000 \\
\hline 15 & $\mathrm{CU}$ & $1 Y Z$ & -0.027163 & 0.214332 & 0.000000 \\
\hline 16 & $\mathrm{CU}$ & $1 \mathrm{xx}$ & -0.660116 & -0.391974 & -1.498876 \\
\hline 17 & $\mathrm{CU}$ & $1 Y Y$ & -0.175358 & 0.480867 & -1.498876 \\
\hline 18 & $\mathrm{CU}$ & $1 \mathrm{ZZ}$ & 0.835474 & -0.088893 & -1.498876 \\
\hline 19 & $\mathrm{CU}$ & $1 X Y$ & -0.087476 & 0.691454 & 0.000000 \\
\hline 20 & $\mathrm{CU}$ & $1 \mathrm{xz}$ & -0.041798 & 0.009567 & 0.000000 \\
\hline 21 & $\mathrm{CU}$ & $1 Y Z$ & 0.027311 & -0.215496 & 0.000000 \\
\hline 22 & $\mathrm{CU}$ & $S$ & 0.000000 & 0.000000 & 0.752270 \\
\hline 23 & $\mathrm{CU}$ & $S$ & 0.000000 & 0.000000 & -0.390430 \\
\hline
\end{tabular}

BETA SET

EIGENVECTORS

1

$-326.7323$ A

1 CU 1 S

2 CU 1 S

3 CU 1 X

4 CU 1 Y

$\begin{array}{llll}5 & \mathrm{CU} & 1 & \mathrm{Z}\end{array}$

6 CU 1 S

7 CU 1 X

8 CU 1 Y

9 CU 1 Z

$10 \mathrm{CU} 1 \mathrm{XX}$

11 CU 1 YY

12 CU $1 \mathrm{ZZ}$

$13 \mathrm{CU} 1 \mathrm{XY}$

14 CU $1 \mathrm{XZ}$

15 CU $1 \mathrm{YZ}$

16 CU $1 \mathrm{XX}$

17 CU 1 YY

18 CU $1 \mathrm{ZZ}$

19 CU 1 XY

20 CU 1 XZ

21 CU $1 \mathrm{YZ}$

22 CU 1 S

23

0.979741

0.074307

0.000000

0.000000

0.000000

0.136528

0.000000

0.000000

0.000000

$-0.065127$

$-0.065127$

$-0.065127$

0.000000

0.000000

0.000000

$-0.014305$

$-0.014305$

$-0.014305$

0.000000

0.000000

0.000000

0.005444

$-0.002694$
6

-4 .

$1 \mathrm{CU} 1 \mathrm{~S}$

2 CU 1 S

3 CU 1 X

4 CU 1 Y

5 CU 1 Z

$6 \mathrm{CU} 1 \mathrm{~S}$

7 CU $1 X$

$8 \mathrm{CU} 1 \mathrm{Y}$

9 CU 1 Z

10 CU $1 \mathrm{XX}$

$11 \mathrm{CU} 1 \mathrm{YY}$

12 CU $1 \mathrm{ZZ}$
A
0.123809

$-0.459947$

0.000000

0.000000

0.000000

1.089820

0.000000

0.000000

0.000000

0.000755

0.000755

0.000755

2
-40.5835
$A$
-0.327467
1.033677
0.000000
0.000000
0.000000
0.000547
0.000000
0.000000
0.000000
0.002957
0.002957
0.002957
0.000000
0.000000
0.000000
0.000737
0.000737
0.000737
0.000000
0.000000
0.000000
-0.000748
0.000415

\section{7}

$-3.1768$

A

0.000000

0.000000

$-0.321652$

$-0.237694$

0.002987

0.000000

0.850966

0.628845

$-0.007902$

0.000000

0.000000

0.000000

3
-35.3420
$A$
0.000000
0.000000
-0.110360
0.259803
0.939605
0.000000
-0.006007
0.014141
0.051141
0.000000
0.000000
0.000000
0.000000
0.000000
0.000000
0.000000
0.000000
0.000000
0.000000
0.000000
0.000000
0.000000
0.000000

8
-3.1768

A

0.000000

0.000000

0.127532

$-0.176793$

$-0.335332$

0.000000

$-0.337399$

0.467723

0.887157

0.000000

0.000000

0.000000

${ }^{4}$
-35.3420
$A$
0.000000
0.000000
0.521414
0.814713
-0.164028
0.000000
0.028380
0.044344
-0.008928
0.000000
0.000000
0.000000
0.000000
0.000000
0.000000
0.000000
0.000000
0.000000
0.000000
0.000000
0.000000
0.000000
0.000000

9

$-3$.

0.000000

0.000000

0.200607

$-0.268726$

0.217970

0.000000

$-0.530726$

0.710943

$-0.576664$

0.000000

0.000000

0.000000
5

$-35.3420$

A

0.000000

0.000000

0.823701

$-0.480916$

0.229721

0.000000

0.044833

$-0.026175$

0.012503

0.000000

0.000000

0.000000

0.000000

0.000000

0.000000

0.000000

0.000000

0.000000

0.000000

0.000000

0.000000

0.000000

0.000000

10 $-0.2836$

A

0.000000

0.000000

0.000000

0.000000

0.000000

0.000000

0.000000

0.000000

0.000000

0.544620

$-0.032916$

$-0.511704$ 


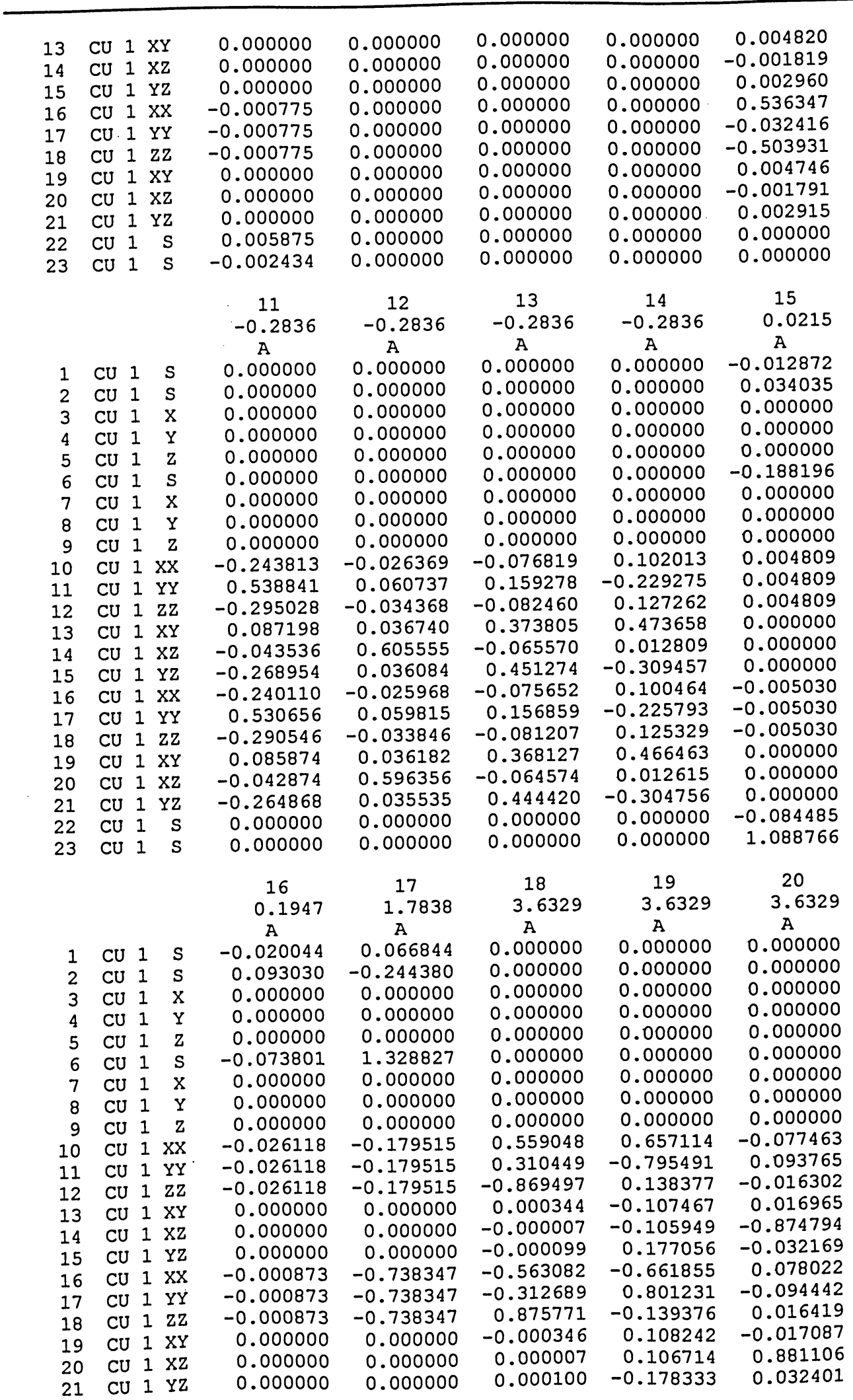




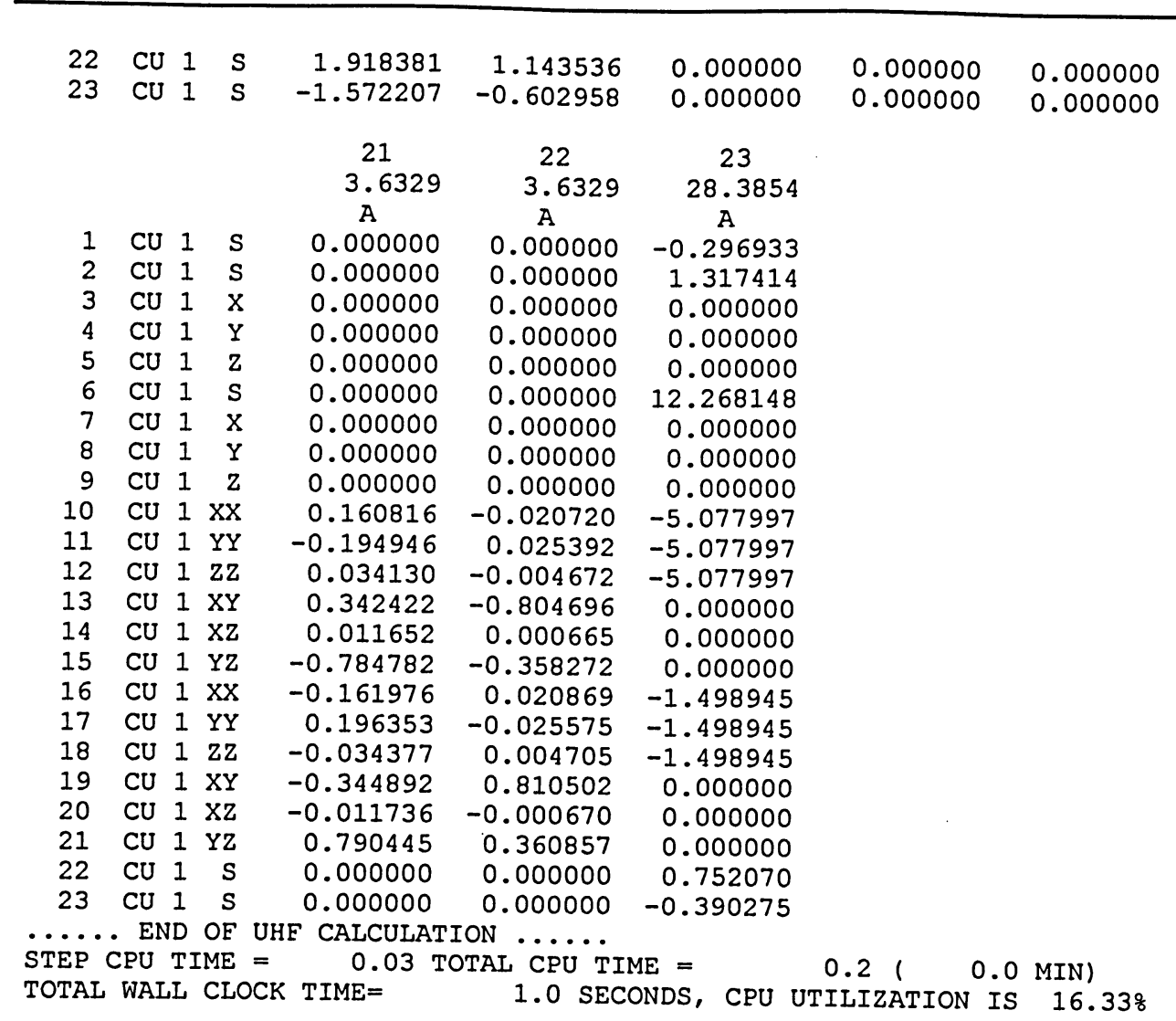

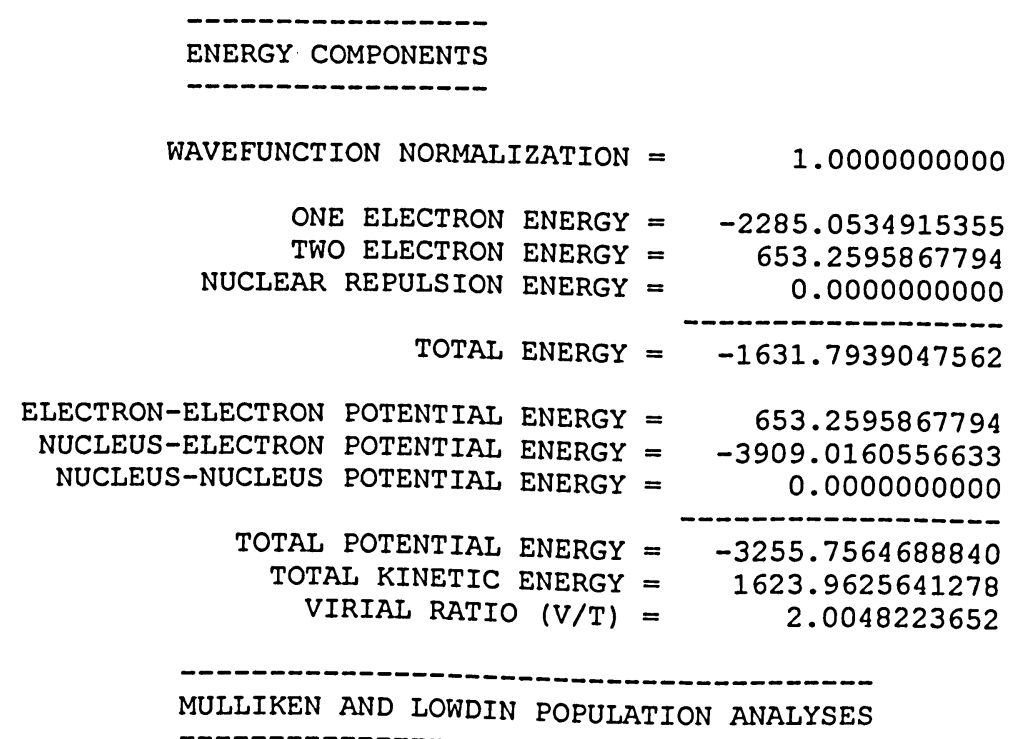

MULIIKEN ATOMIC POPULATION IN EACH MOLECULAR ORBITAL ALPHA ORBITALS

1

1.000000
2

1.000000
3

1.000000
4

1.000000
5

1.000000 


$$
1.000000
$$

6

$$
1.000000
$$

1.000000

1.000000

7

1.000000

12

11

1.000000

1.000000

1.000000

1.000000

1.000000

1.000000
1.000000

9

1.000000

1.000000

14

1.000000

1.000000

1.000000

MULIIKEN ATOMIC POPULATION IN EACH MOLECULAR ORBITAL BETA ORBITALS

\begin{tabular}{|c|c|c|c|c|}
\hline \multicolumn{2}{|c|}{$\cdots$} & \multicolumn{2}{|c|}{$\begin{array}{c}\text { - POPULATIONS IN EAC } \\
\text { MULIIKEN }\end{array}$} & $\begin{array}{l}\text { AO ---- } \\
\text { LOWDIN }\end{array}$ \\
\hline 1 & $\mathrm{CU}$ & $1 \mathrm{~S}$ & 1.99120 & 1.99566 \\
\hline 2 & CU & $1 \mathrm{~S}$ & 2.01053 & 1.93780 \\
\hline 3 & $\mathrm{CU}$ & $1 x$ & 2.00000 & 2.00000 \\
\hline 4 & $\mathrm{CU}$ & $1 Y$ & 2.00000 & 2.00000 \\
\hline 5 & $\mathrm{CU}$ & 12 & 2.00000 & 2.00000 \\
\hline 6 & $\mathrm{CU}$ & $1 \mathrm{~s}$ & 1.99873 & 0.80434 \\
\hline 7 & $\mathrm{CU}$ & $1 x$ & 2.00000 & 2.00000 \\
\hline 8 & $\mathrm{CU}$ & $1 Y$ & 2.00000 & 2.00000 \\
\hline 9 & $\mathrm{CU}$ & 12 & 2.00000 & 2.00000 \\
\hline 10 & $\mathrm{CU}$ & $1 \mathrm{xx}$ & 0.67368 & 0.99771 \\
\hline 11 & $\mathrm{CU}$ & $1 \mathrm{YY}$ & 0.67368 & 0.99771 \\
\hline 12 & $\mathrm{CU}$ & $1 \mathrm{zz}$ & 0.67368 & 0.99771 \\
\hline 13 & $\mathrm{CU}$ & $1 X Y$ & 1.00986 & 1.00920 \\
\hline 14 & $\mathrm{CU}$ & $1 \mathrm{xz}$ & 1.00986 & 1.00920 \\
\hline 15 & CU & $1 \mathrm{YZ}$ & 1.00986 & 1.00920 \\
\hline 16 & CU & $1 x x$ & 0.65902 & 0.76591 \\
\hline 17 & $\mathrm{CU}$ & $1 Y Y$ & 0.65902 & 0.76591 \\
\hline 18 & $\mathrm{CU}$ & $1 \mathrm{zz}$ & 0.65902 & 0.76591 \\
\hline
\end{tabular}

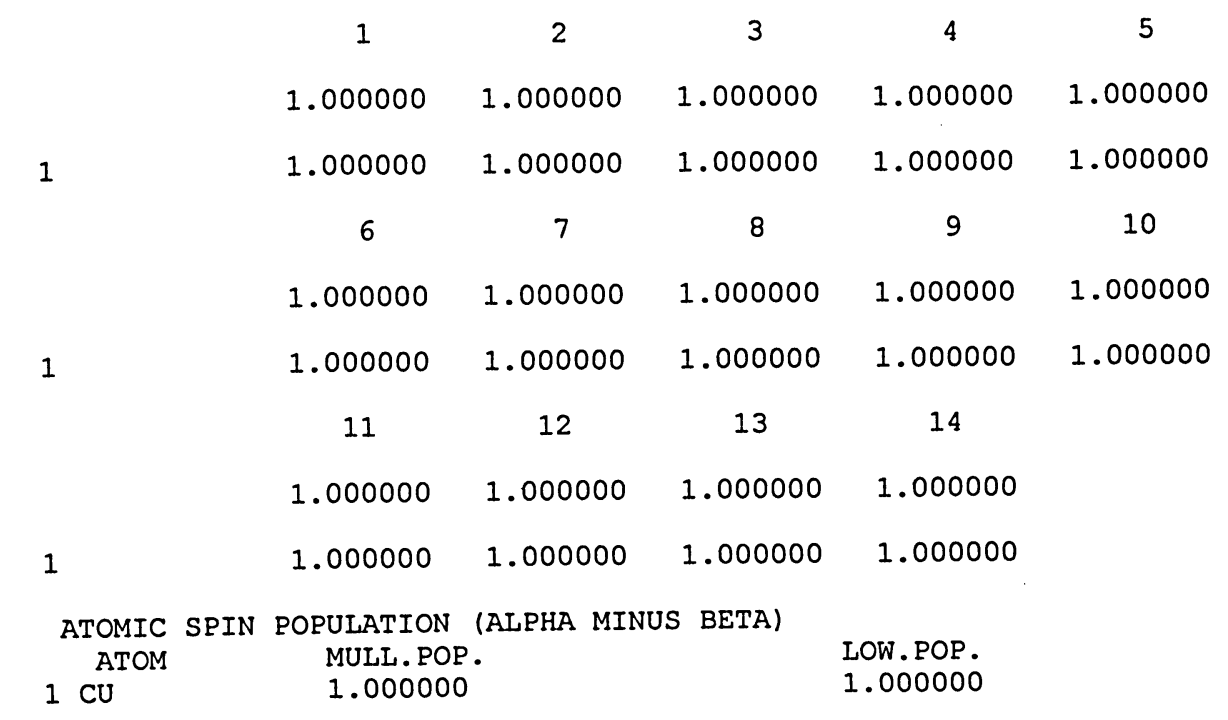




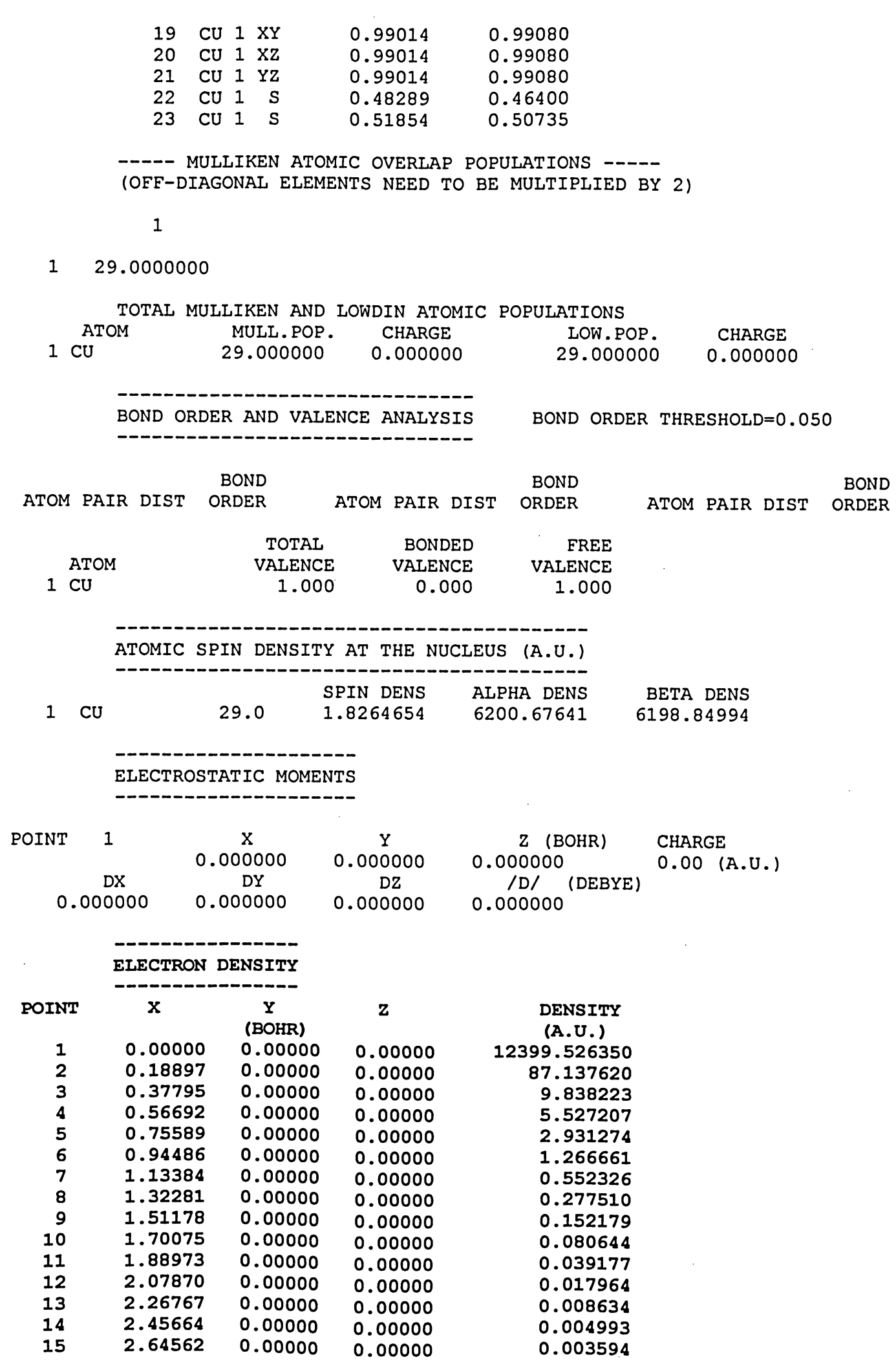




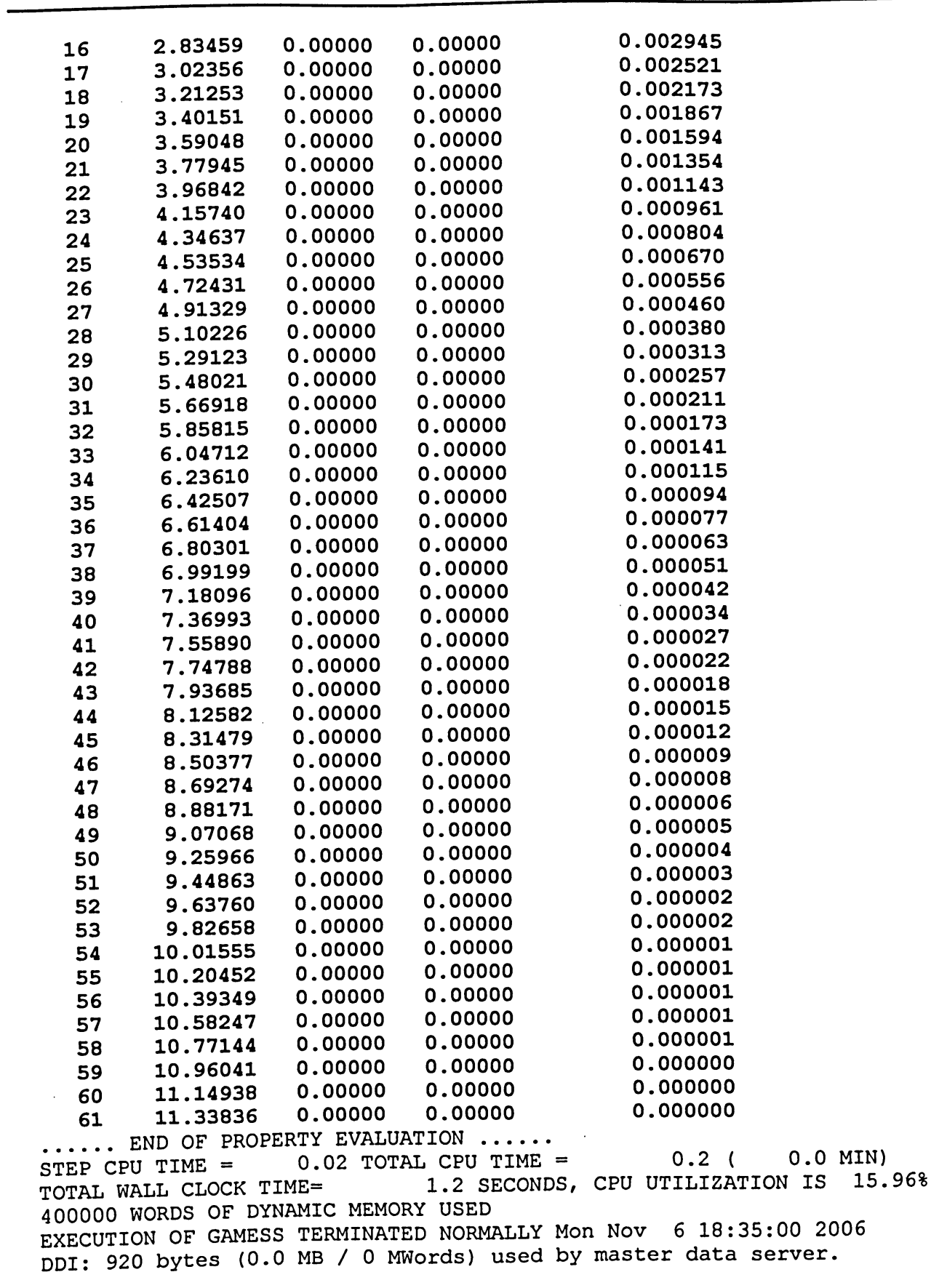

CPU timing information for all processes $==================================$ $0: 0.187000+0.046000=0.233000$ 1: $0.031000+0.000000=0.031000$

ddikick.exe: exited gracefully. -.-- accounting info - - - 
The electron density is presented in bold face towards the end of the output. The native units of WinGAMESS for distance and electron density are Bohr and Coulomb per cubic Bohr respectively. Note that $1 \mathrm{Bohr}$ is 0.529189 Angstroms. If the distance in the output is converted to Angstroms and the electron density in the output is converted to Coulomb per cubic Angstrom, then the curve in Figure 3 (Section 3.5) is obtained. 


\section{APPENDIX E: CLASSICAL STATISTICAL MECHANICS AND THE MAXWELL-BOLTZMANN DISTRIBUTION LAW}




\section{E1. Introduction}

Statistical mechanics is the branch of science that deals with providing reasonable methods for treating the behaviour of a mechanical system regarding whose condition or state we have incomplete knowledge. Most other branches of science allow precise predictions of the future state of the system to be made from precise knowledge of its condition at the present. However, the limitations of this philosophy are realized when the number of particles in the system becomes very large, or when the initial state of the system is only partially known. Statistical mechanics then provides a framework for making reasonable predictions of the future state of the system (which can be expected to hold on an average) from the partial information available on the initial state. This is achieved by studying the behaviour of an ensemble, or collection, of systems that are similar in structure to the system of interest, and are distributed over a range of different precise states. Such a suitable ensemble is called a representative ensemble for the system. The average behaviour of the systems in the representative ensemble is studied and is used to make predictions about the average expected behaviour of the system at hand. The following sections present some details on statistical mechanics and conclude with a very important result of classical statistical mechanics: the Maxwell-Boltzmann distribution for states of the system. Information for this appendix has been obtained from [33].

\section{E2. Concept of Phase-Space}

The state of mechanical systems is completely determined by two sets of quantities: the coordinates (of degrees of freedom) of the system, and the momentum (or velocities) of the various constituents of the system. For a system of $n$ particles, each with $r$ degrees of freedom, the state can be represented by a point in a hypothetical $2 n r$ dimensional space, with $n r$ dimensions for the coordinates and $n r$ dimensions for the momentum. This hypothetical $2 n r$ dimensional space is called the phase-space of the system, with the $n r$ 
dimensions for the coordinates constituting the configuration-space and the $n r$ dimensions for the momentum constituting the momentum-space. The phase-space for a single particle of a system has been termed $\mu$-space in statistical mechanics, while the phase-space for the entire system has been termed $\gamma$-space. The state of a particle is therefore a single point in $\mu$-space, while the state of the system is either a single point in $\gamma$-space, or a collection of points in $\mu$-space with each point representing one particle of the system. Analogously, the state of an ensemble is a collection of points in $\gamma$-space with each point representing one system of the ensemble.

The number of systems in the ensemble is usually assumed to be large. In that case then, the number of systems having coordinates and momentum in a certain range can be represented by a density, $\rho$, of points in $\gamma$-space. Hence the number of systems having positions and momentum between $q_{1}, q_{2}, \ldots, q_{f}, p_{1}, p_{2}, \ldots, p_{f}$ and $q_{1}+\delta q_{1}, q_{2}+\delta q_{2}, \ldots$, $q_{f}+\delta q_{f}, p_{1}+\delta p_{1}, p_{2}+\delta p_{2}, \ldots, p_{f}+\delta p_{f}$, where $q$ denotes coordinates and $p$ denotes momentum, is:

$$
\delta N=\rho\left(q_{1}, q_{2}, \ldots, q_{f}, p_{1}, p_{2}, \ldots, p_{f}, t\right) \delta q_{1} \delta q_{2} \ldots \delta q_{f} \delta p_{1} \delta p_{2} \ldots \delta p_{f}
$$

\section{E3. Statistical Ensembles in Classical Statistical Mechanics}

\section{E3.1 Uniform Ensemble}

A uniform ensemble is an ensemble in statistical equilibrium (meaning that $\rho$ does not change with time locally), with:

$$
\rho=\text { constant }
$$

everywhere in phase-space. Uniform ensembles are used to represent systems regarding which no information is available. 


\section{E3.2 Microcanonical Ensemble}

A microcanonical ensemble is an ensemble in statistical equilibrium with:

$$
\begin{aligned}
& \rho=\text { constant (for energy between } \mathrm{E} \text { and } \mathrm{E}+\delta \mathrm{E} \text { ) } \\
& \rho=0 \text { (for energy outside the above range) }
\end{aligned}
$$

Microcanonical ensembles are used to represent systems whose initial energies are precisely known and conserved.

\section{E3.3 Canonical Ensemble}

A canonical ensemble is an ensemble in statistical equilibrium with:

$$
\rho=N \exp \left(\frac{\psi-E}{\theta}\right)
$$

, where $E$ is the energy of the system, and $\psi$ and $\theta$ are parameters independent of position and momentum whose values complete the description of the distribution. Canonical ensembles are used to represent systems whose initial energies are close to a precisely known value.

\section{E4. Maxwell-Boltzmann Distribution Law}

The initial energy of most systems is usually precisely known. Hence a microcanonical ensemble can be used as a representative ensemble for the system. Let $n_{i}$ be the number of particles of a $n$ particle system whose coordinates and momentum lie in cell $i$ of $\mu$ space. Then the probability of finding the system in this condition is (assuming equal $a$ priori probabilities for finding a particle in a cell of $\mu$-space): 


$$
P=\frac{n !}{n_{1} ! n_{2} ! n_{3} ! \ldots n_{i} ! \ldots} \times \text { constant }
$$

, where the constant normalizes the probability and is independent of the condition of the system. The condition of maximum probability is obtained by maximizing (E5) subject to the following logical constraints:

$$
\delta n=\sum_{i} \delta n_{i}=0
$$

, and:

$$
\delta E=\sum_{i} \varepsilon_{i} \delta n_{i}=0
$$

Descriptively, the constraints mean that the total number of particles in the system and the total system energy (with $\varepsilon_{i}$ being the energy associated with cell $i$ of $\mu$-space) are constant (this was the reason why a microcanonical ensemble was chosen to begin with). The maximization procedure and ancillary considerations lead to the Maxwell-Boltzmann distribution law governing the condition of maximum probability:

$$
n_{i}=A \exp \left(-\frac{\varepsilon_{i}}{k_{B} T}\right)
$$

$A$ is a normalization constant, $k_{B}$ is Boltzmann's constant and $T$ is the absolute temperature of the system. In differential form, the Maxwell-Boltzmann distribution law gives the number of particles in any region of $\mu$-space with energy $\varepsilon$ as:

$$
\delta n=n C \exp \left(-\frac{\varepsilon_{i}}{k_{B} T}\right)\left\{\delta q_{1} \delta q_{2} \ldots \delta q_{r} \delta p_{1} \delta p_{2} \ldots \delta p_{r}\right\}
$$


, where $C$ is another normalization constant given by:

$$
C=\frac{1}{\int \ldots \int \exp \left(-\frac{\varepsilon_{i}}{k_{B} T}\right) d q_{1} d q_{2} \ldots d q_{r} d p_{1} d p_{2} \ldots d p_{r}}
$$

\section{E5. Maxwell Distribution Law for Velocities}

A special case of the Maxwell-Boltzmann distribution law arises for identical particles in a conservative field. In this case, the potential energy is dependent on the coordinates alone, while the kinetic energy is quadratically dependent on the momentum (or velocities) alone. Hence, using Cartesian coordinates, $\varepsilon$ can be written as:

$$
\varepsilon=\varepsilon_{V}(x, y, z)+\frac{1}{2} m\left(\dot{x}^{2}+\dot{y}^{2}+\dot{z}^{2}\right)
$$

Substituting (E11) into (E9) and integrating out the coordinates (from $-\infty$ to $\infty$ ) gives:

$$
\delta n=n B \exp \left(-\frac{m\left(\dot{x}^{2}+\dot{y}^{2}+\dot{z}^{2}\right)}{2 k_{B} T}\right) \delta \dot{x} \delta \dot{y} \delta \dot{z}
$$

as the number of particles of the system having velocity components in a range $\delta \dot{x} \delta \dot{y} \delta \dot{z}$. The normalization constant $B$ can easily be determined as follows:

$$
n=\int \delta n=n B \int_{-\infty}^{\infty} \int_{-\infty}^{\infty} \int_{-\infty}^{\infty} \exp \left(-\frac{m\left(\dot{x}^{2}+\dot{y}^{2}+\dot{z}^{2}\right)}{2 k_{B} T}\right) d \dot{x} d \dot{y} d \dot{z}
$$




$$
\Rightarrow B=\frac{1}{\int_{-\infty}^{\infty} \int_{-\infty}^{\infty} \int_{-\infty}^{\infty} \exp \left(-\frac{m\left(\dot{x}^{2}+\dot{y}^{2}+\dot{z}^{2}\right)}{2 k_{B} T}\right) d \dot{x} d \dot{y} d \dot{z}}=\left(\frac{m}{2 \pi k_{B} T}\right)^{\frac{3}{2}}
$$

Note that the following integral was used for performing the integration in (E14):

$$
\int_{-\infty}^{\infty} \exp \left(-a s^{2}\right) d s=\sqrt{\frac{\pi}{a}}
$$

Substituting (E14) into (E12) gives the Maxwell distribution law for velocities:

$$
\delta n=n\left(\frac{m}{2 \pi k_{B} T}\right)^{\frac{3}{2}} \exp \left(-\frac{m\left(\dot{x}^{2}+\dot{y}^{2}+\dot{z}^{2}\right)}{2 k_{B} T}\right) \delta \dot{x} \delta \dot{y} \delta \dot{z}
$$

The distribution of one component of velocity, say the X-component, is obtained by integrating out the other two components of velocity (from $-\infty$ to $\infty$ ). Hence:

$$
\begin{aligned}
& \delta n_{\dot{x}}=n \sqrt{\frac{m}{2 \pi k_{B} T}} \exp \left(-\frac{m \dot{x}^{2}}{2 k_{B} T}\right) \delta \dot{x} \\
& \delta n_{\dot{y}}=n \sqrt{\frac{m}{2 \pi k_{B} T}} \exp \left(-\frac{m \dot{y}^{2}}{2 k_{B} T}\right) \delta \dot{y} \\
& \delta n_{\dot{z}}=n \sqrt{\frac{m}{2 \pi k_{B} T}} \exp \left(-\frac{m \dot{z}^{2}}{2 k_{B} T}\right) \delta \dot{z}
\end{aligned}
$$

Since $\dot{x}$ is the same as $v_{x}$ and similar notations hold for the Y- and Z- components of velocity, the probability distributions for equation (E17) are simply: 


$$
\begin{aligned}
& f\left(v_{x}\right)=\sqrt{\frac{m}{2 \pi k_{B} T}} \exp \left(-\frac{m v_{x}{ }^{2}}{2 k_{B} T}\right) \\
& f\left(v_{y}\right)=\sqrt{\frac{m}{2 \pi k_{B} T}} \exp \left(-\frac{m v_{y}{ }^{2}}{2 k_{B} T}\right) \\
& f\left(v_{z}\right)=\sqrt{\frac{m}{2 \pi k_{B} T}} \exp \left(-\frac{m v_{z}{ }^{2}}{2 k_{B} T}\right)
\end{aligned}
$$

The speed of a particle, $v$, is given by the following expression:

$$
v=\sqrt{\dot{x}^{2}+\dot{y}^{2}+\dot{z}^{2}}
$$

If the velocity components are in the infinitesimal range $\delta \dot{x} \delta \dot{y} \delta \dot{z}$, then the corresponding infinitesimal range of speed $v$ is:

$$
\delta \dot{x} \delta \dot{y} \delta \dot{z}=\frac{4}{3} \pi\left[(v+\delta v)^{3}-v^{3}\right] \approx 4 \pi v^{2} \delta v
$$

, neglecting higher order terms. Using equations (E19) and (E20), equation (E16) can then be rewritten for the number of particles having speed between $v$ and $v+\delta v$ as:

$$
\delta n_{v}=4 \pi n\left(\frac{m}{2 \pi k_{B} T}\right)^{\frac{3}{2}} \exp \left(-\frac{m v^{2}}{2 k_{B} T}\right) v^{2} \delta v
$$

The average speed of particles is then:

$$
\bar{v}=\frac{\int_{0}^{\infty} v \delta n_{v}}{\int_{0}^{\infty} \delta n_{v}}=4 \pi\left(\frac{m}{2 \pi k_{B} T}\right)^{\frac{3}{2}} \int_{0}^{\infty} \exp \left(-\frac{m v^{2}}{2 k_{B} T}\right) v^{3} \delta v=\sqrt{\frac{8 k_{B} T}{\pi m}}
$$


Note that even thought the average speed is not zero, the average values of the velocity components are zero because the velocity components are integrated from $-\infty$ to $\infty$, unlike the speed which is always non-negative.

Similarly, the average kinetic energy of particles is:

$$
K . E_{\text {avg. }}=\frac{\int_{0}^{\infty} \frac{1}{2} m v^{2} \delta n_{v}}{\int_{0}^{\infty} \delta n_{v}}=2 \pi m\left(\frac{m}{2 \pi k_{B} T}\right)^{\frac{3}{2}} \int_{0}^{\infty} \exp \left(-\frac{m v^{2}}{2 k_{B} T}\right) v^{4} \delta v=\frac{3}{2} k_{B} T
$$

Using the principle of equipartition of energy in statistical mechanics, the average kinetic energy per axis is then:

$$
\frac{1}{2} m\left(v_{x}{ }^{2}\right)_{\text {avg. }}=\frac{1}{2} m\left(v_{y}{ }^{2}\right)_{\text {avg. }}=\frac{1}{2} m\left(v_{z}{ }^{2}\right)_{\text {avg. }}=\frac{1}{2} k_{B} T
$$




\section{REFERENCES}

[1] Mazzone, A. M., 2004. Silicon nanocrystals studied using Hartree-Fock and molecular dynamics. Applied Surface Science (226) 19-25.

[2] Hara, S., Izumi, S., Kumagai, T., Sakai, S., 2005. Surface energy, stress and structure of well-relaxed amorphous silicon: A combination approach of ab initio and classical molecular dynamics. Surface Science (585) 17-24.

[3] Muralidharan, K., Simmons, J. H., Deymier, P. A., Runge, K., 2005. Molecular dynamics studies of brittle fracture in vitreous silica: Review and recent progress. Journal of Non-Crystalline Solids (351) 1532-1542.

[4] Kumar, K. S., Van Swygenhoven, H., Suresh, C., 2003. Mechanical behavior of nanocrystalline metals and alloys. Acta Materialia (51) 5743-5774.

[5] Van Swygenhoven, H., Caro, A., 1997. Plastic behaviour of nanophase Ni: A molecular dynamics computer simulation. American Institute of Physics (97) S0003-6951 03838-2.

[6] Derlet, P. M., Van Swygenhoven, H., 2002. The role played by two parallel free surfaces in the deformation mechanism of nanocrystalline metals: a molecular dynamics simulation. Philosophical Magazine A (82) 1-15.

[7] Chocyk, D., Zientarski, T., Proszynski, A., Pienkos, T., Gladyszewski, L., Gladyszewski, G., 2005. Evolution of stress and structure in $\mathrm{Cu}$ thin films. Cryst. Res. Technol. (40) 509-516. 
[8] Zhang, S., Johnson, H. T., Wagner, G. J., Liu, W. K., Hsia, K. J., 2003. Stress generation mechanisms in carbon thin films grown by ion-beam deposition. Acta Materialia (51) 5211-5222.

[9] Buch, V., Martoňák, R., Parrinello, M., 2005. A new molecular-dynamics based approach for molecular crystal structure search. The Journal of Chemical Physics (123) 051108.

[10] English, N. J., 2005. Molecular dynamics simulations of liquid water using various long-range electrostatics techniques. Molecular Physics (103) 1945-1960.

[11] Kamberaj, H., Low, R. J., Neal, M. P., 2005. Time reversible and symplectic integrators for molecular dynamics simulations of rigid molecules. The Journal of Chemical Physics (122) 224114.

[12] Yamauchi, Y., Nakai, H., 2005. Hybrid approach for ab initio molecular dynamics simulation combining energy density analysis and short-time Fourier transform: Energy transfer spectrogram. The Journal of Chemical Physics (123) 034101.

[13] Ivanov, S. D., Lyubartsev, A. P., 2005. Simulations of one- and two-electron systems by Bead-Fourier path integral molecular dynamics. The Journal of Chemical Physics (123) 034105.

[14] Liu, W. K., Karpov, E. G., Zhang, S., Park, H. S., 2004. An introduction to computational nanomechanics and materials. Computer Methods in Applied Mechanics and Engineering (193) 1529-1578.

[15] Hohenberg, P., Kohn, W., 1964. Inhomogeneous electron gas. Physical Review (136) B864-B871. 
[16] Kohn, W., Sham, L. J., 1965. Self-consistent equations including exchange and correlation effects. Physical Review (140) A1133-A1138.

[17] Daw, M. S., Baskes, M. I., 1983. Semiempirical, Quantum Mechanical Calculation of Hydrogen Embrittlement in Metals. Physical Review Letters (50) 1285-1288.

[18] Daw, M. S., Baskes, M. I., 1984. Embedded-atom method: Derivation and application to impurities, surfaces, and other defects in metals. Physical Review B (29) 6443-6453.

[19] Foiles, S. M., Baskes, M. I., Daw, M. S., 1986. Embedded-atom-method functions for the fcc metals $\mathrm{Cu}, \mathrm{Ag}, \mathrm{Au}, \mathrm{Ni}, \mathrm{Pd}, \mathrm{Pt}$, and their alloys. Physical Review B (33) 7983-7991.

[20] Plimpton, S. J., Hendrickson, B. A., 1993. Parallel Molecular Dynamics with the Embedded Atom Method. Materials Theory and Modelling (edited by Broughton, J., Bristowe, P., Newsam, J.), MRS Proceedings 291, Pittsburgh, PA, pp. 37.

[21] Haile, J. M., 1997. Molecular Dynamics Simulation: Elementary Methods. WileyInterscience.

[22] Clausius, R. J. E., 1870. On a mechanical theorem applicable to heat. Phil. Mag. (40) 122-127.

[23] Park, H. S., Zimmerman, J. A., 2005. Modeling inelasticity and failure in gold nanowires. Physical Review B (72), 054106. 
[24] Zhou, M., 2003. A new look at the atomic level virial stress: on continuummolecular system equivalence. Royal Society of London Proceedings Series A, Vol. 459, Issue 2037, pp.2347-2392.

[25] Zhang, S., Johnson, H. T., Wagner, G. J., Liu, W. K., Hsia, K. J., 2003. Stress generation mechanisms in carbon thin films grown by ion-beam deposition. Acta Materialia (51) 5211-5222.

[26] Wu, E. Y., 1990. A method for treating thermal expansion effects in molecular dynamics simulations for solids. Condensed Matter 2, 9335-9344.

[27] Dieter, G. E., 1988. Mechanical Metallurgy - SI Metric Edition. McGraw-Hill, London.

[28] Y Touloukian, Y. S., Kirby, R. K., Taylor, R. E., Desai, P. D., 1970. Thermophysical Properties of Matter, Vol. 12. IFI/Plenum.

[29] Huntington, H. B., 1958. The Elastic Constants of Crystals. Academic Press, Inc.

[30] Overton, W. C., Jr., Gaffney, J., 1955. Temperature Variation of the Elastic Constants of Cubic Element. I. Copper. Physical Review (98) 969-977.

[31] Pauling, L., Wilson, E. B., 1935. Introduction to Quantum Mechanics with Applications to Chemistry. McGraw-Hill, London.

[32] Cook, D. B., 1998. Handbook of Computational Quantum Chemistry. Dover Publications, Inc., New York. 
[33] Tolman, R. C., 1979. The Principles of Statistical Mechanics. Dover Publications, Inc., New York. 